\title{
Role of Pax6 in pancreatic endocrine cell subtype specification
}

\author{
Dissertation \\ In partial fulfillment of the requirements for the degree of \\ "Doctor of Philosophy (Ph.D.)" \\ in the Molecular Medicine study program \\ at the Faculty of Medicine, \\ Georg-August University Göttingen
}

Submitted by

Zeeshan Ahmad

Born in

Lahore, Pakistan

Göttingen, March 2013 
Dedicated to

My Father

Whose love and support has enabled me to reach this destination 
Thesis Supervisor:

Prof. Dr. Ahmed Mansouri

Thesis Committee Members:

Prof. Dr. Ahmed Mansouri

Department of Molecular Cell Biology

Max Planck Institute for Biophysical Chemistry, Göttingen

Prof. Dr. Wolfgang Engel

Department of Human Genetics

Georg-August University, Göttingen

Prof. Dr. Ralf Dressel

Department of Cellular and Molecular Immunology

Georg-August University, Göttingen

Date of thesis submission: March 28, 2013

Date of oral examination: May 17, 2013 


\section{Affidavit}

I hereby declare that my doctoral thesis entitled "Role of Pax6 in pancreatic endocrine cell subtype specification" has been written independently with no other sources and aids than quoted.

Zeeshan Ahmad

Göttingen, March 28, 2013

This work has been carried out at the Max Planck Institute for Biophysical Chemistry (Karl Friedrich Bonhoefer Institute) in Göttingen in the group of Prof. Dr. Ahmed Mansouri (Molecular Cell Differentiation). 


\section{Table of contents}

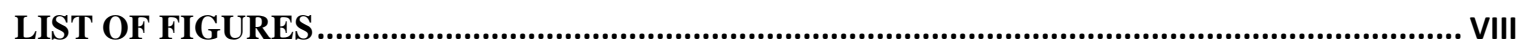

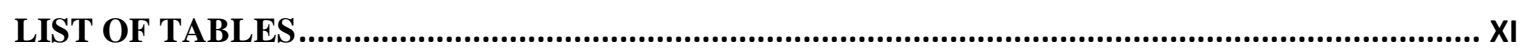

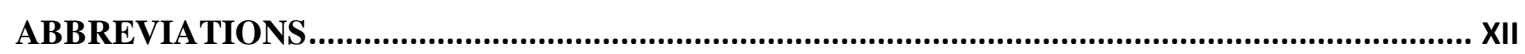

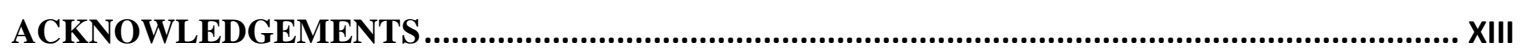

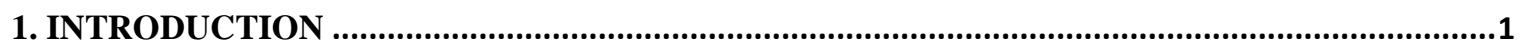

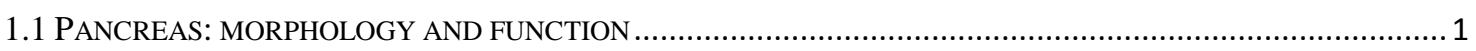

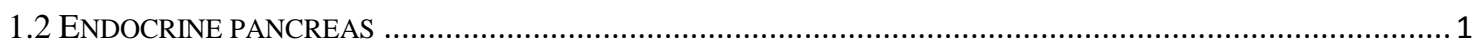

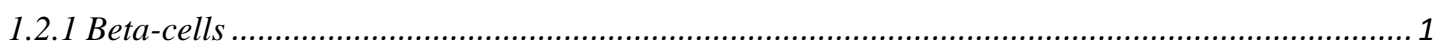

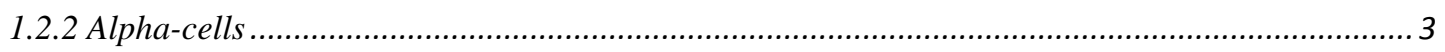

1.2.3 Delta-Cells

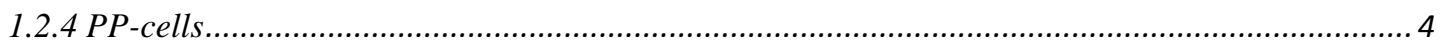

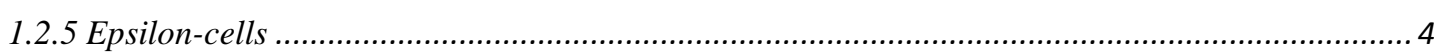

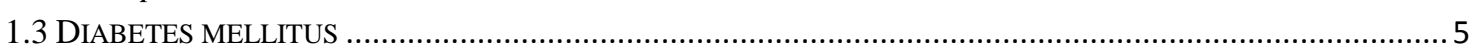

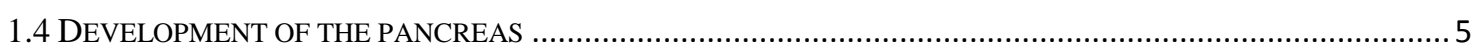

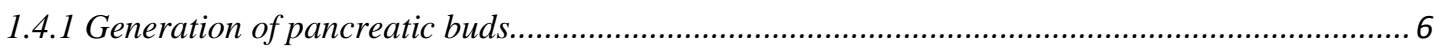

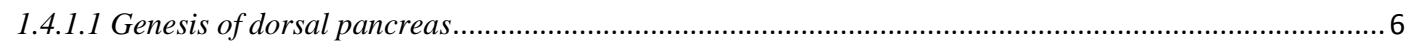

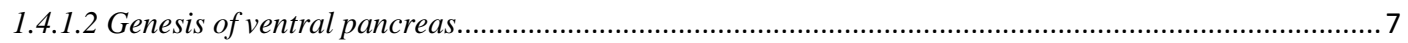

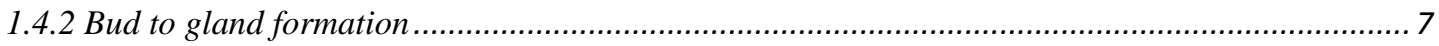

1.5 ROLE OF TRANSCRIPTION FACTORS IN DEVELOPING AND ADULT ENDOCRINE PANCREAS ....................... 8

$1.5 .1 \mathrm{Hlxb9}$

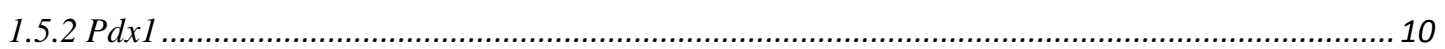

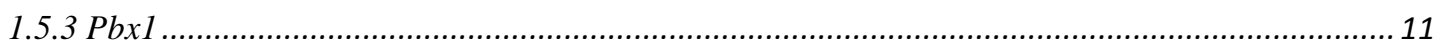

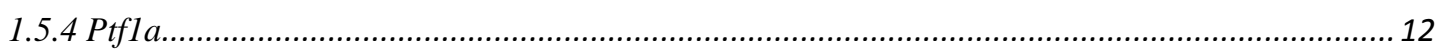

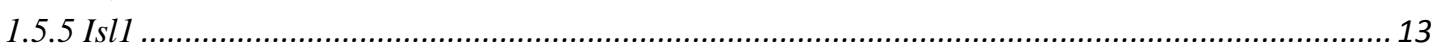

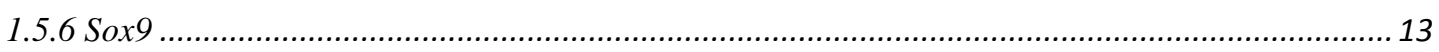

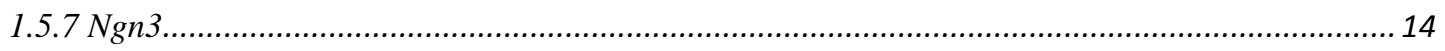

1.5 .8 Rfx6

1.5.9 NeuroD

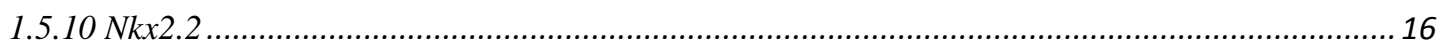

$1.5 .11 \mathrm{Nkx6.1}$

1.5.12 Pax4 and Arx

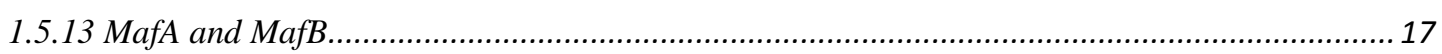

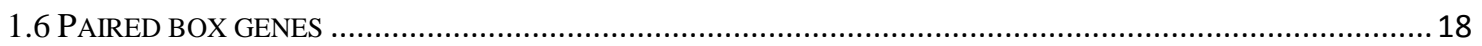

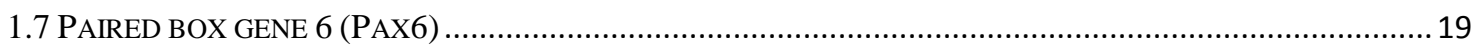

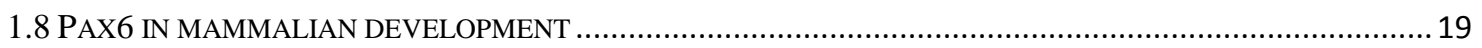

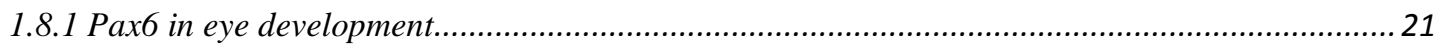

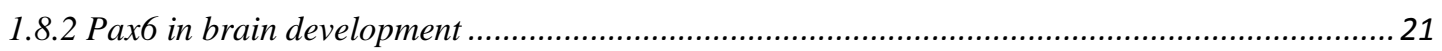

1.8.3 Pax6 in Pancreas development...................................................................................... 22

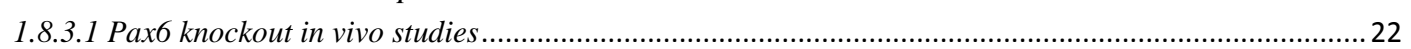

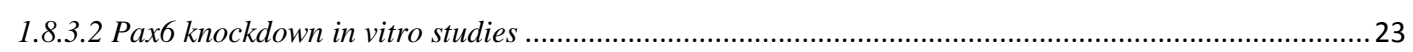

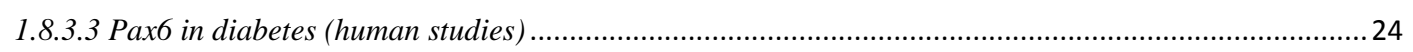

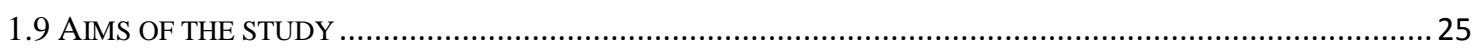

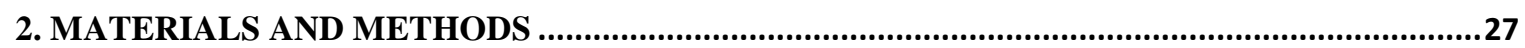




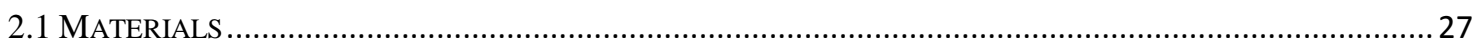

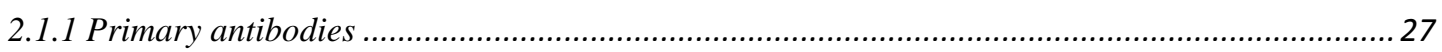

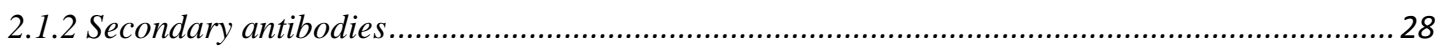

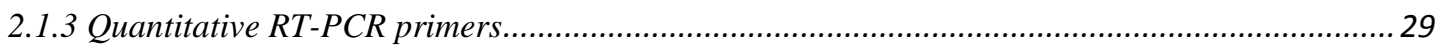

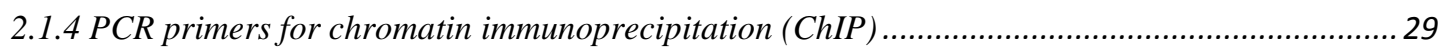

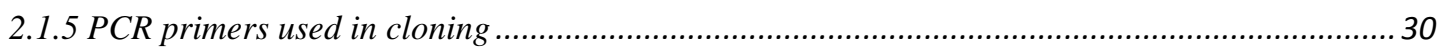

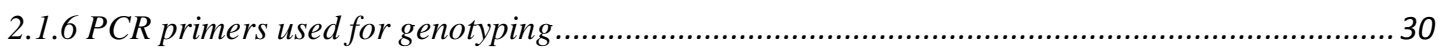

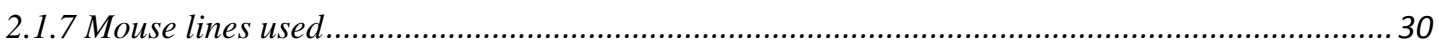

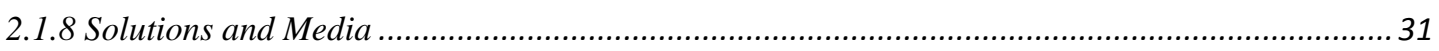

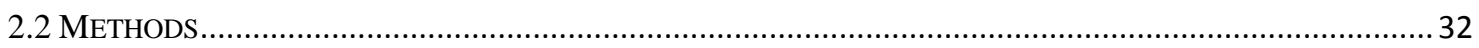

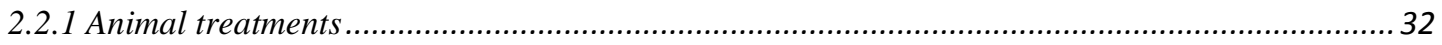

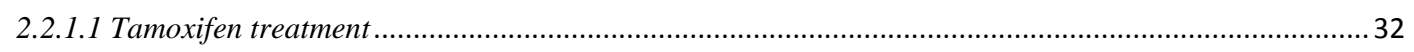

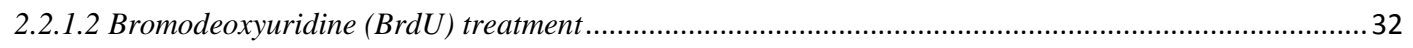

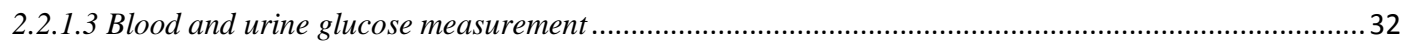

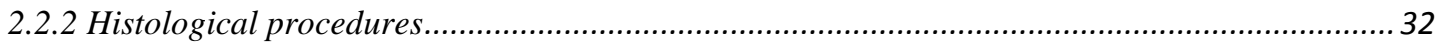

2.2.2.1 Pancreas preparation, cryo-embedding, and sectioning ………..................................................... 32

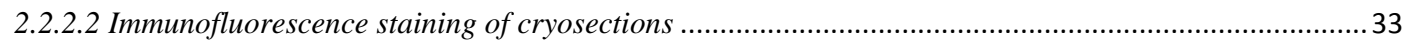

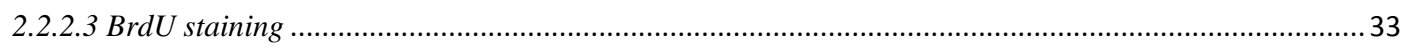

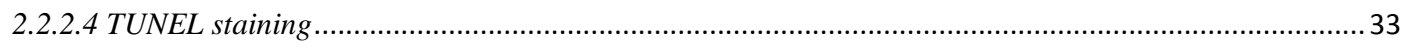

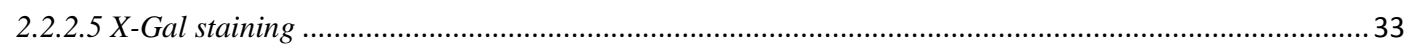

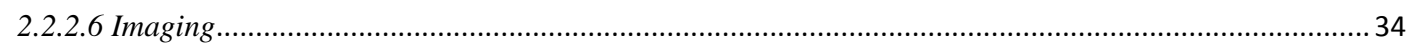

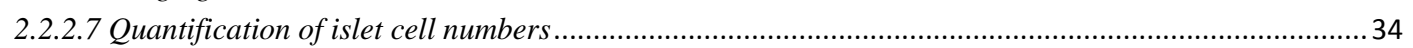

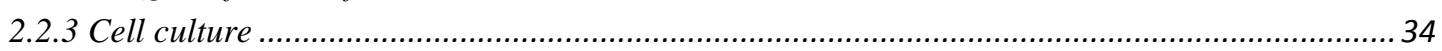

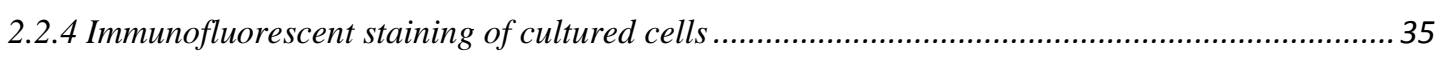

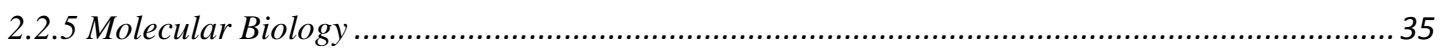

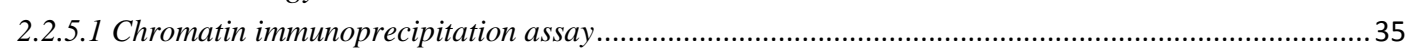

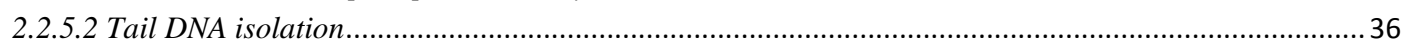

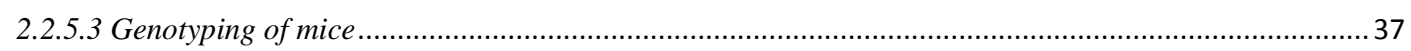

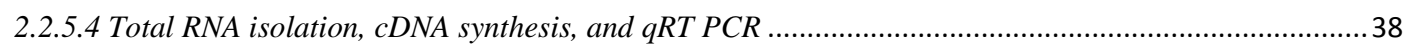

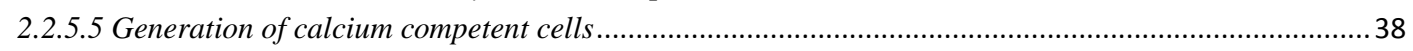

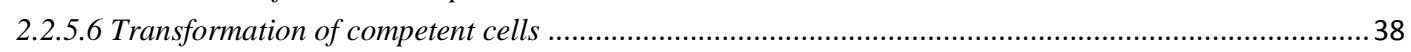

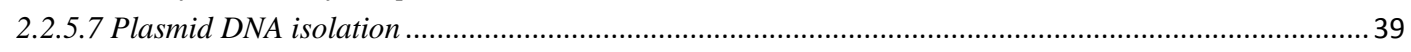

2.2.5.8 Agarose gel electrophoresis and gel extraction of DNA fragments .....................................................39

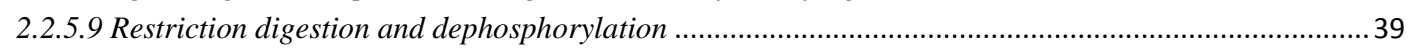

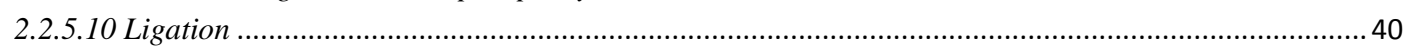

2.2.5.11 Generation of Pax6 overexpression construct ...................................................................... 40

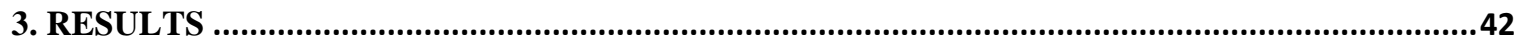

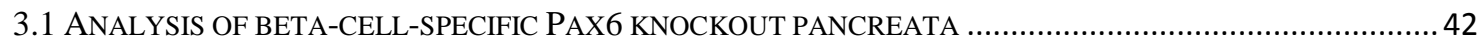

3.1.1 Inducible conditional knockout of Pax6 in the adult pancreatic beta-cells ................................. 42

3.1.2 Development of diabetes in the beta-cell-specific Pax6 KO mice............................................. 42

3.1.3 Expression of pancreatic endocrine hormones in the beta-cell-specific Pax6 KO mice................ 45

3.1.4 Expression of beta-cell related transcription factors in the pancreata of beta-cell-specific Pax6

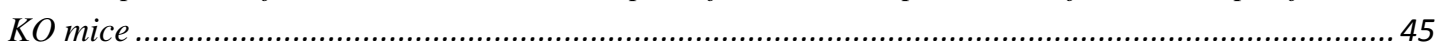

3.1.5 Expression of Glut2 and GLP-1 receptor is lost after ablation of Pax6 in beta-cells ..................52

3.1.6 Defective proinsulin processing in Pax6-deficient beta-cells ..................................................... 52

3.1.7 Ghrelin ${ }^{+}$cells originate from beta-cells in the beta-cell-specific Pax6 KO mice ..........................55

3.1.8 Tracing the fate of Pax6-deficient beta-cells over long period of time....................................... 61

3.1.9 Ghrelin and Glut2 are not the direct downstream targets of Pax6............................................6

3.1.10 Beta-cell regeneration in the beta-cell-specific Pax6 KO islets .............................................69 
3.2 ANALYSIS OF ALPHA-CELL-SPECIFIC PAX6 KNOCKOUT PANCREATA …................................................. 73

3.2.1 Conditional knockout of Pax6 in the pancreatic alpha-cells ...................................................... 73

3.2.2 Ghrelin $^{+}$cells originate from alpha-cells in the alpha-cell-specific Pax6 KO pancreata ............ 76

3.2.2.1 Co-expression of ghrelin with glucagon in the alpha-cell-specific Pax6 KO pancreata ........................76

3.2.2.2 Expression of alpha-cell related transcription factors in the ghrelin ${ }^{+}$cell population of alpha-cellspecific Pax6 KO islets.

3.2.3 Regeneration of alpha-cells in the alpha-cell-specific Pax6 KO islets....................................... 81

3.2.4 7B2 is upregulated and PC2 not affected in the alpha-cell-specific Pax6 KO islets ....................85

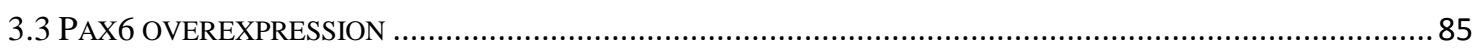

3.3.1 Generation of transgenic mice conditionally overexpressing Pax6 .......................................... 85

3.3.2 Pancreatic phenotype of Pax6 overexpressing mice.............................................................. 88

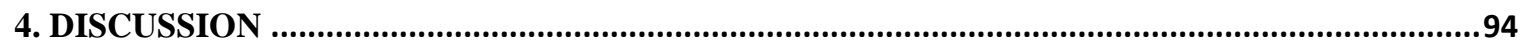

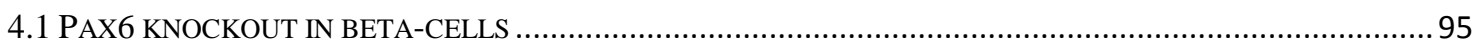

4.1.1 Hyperglycemia in beta-cell-specific Pax6 KO mice and the associated changes in the pancreatic endocrine cell population ...................................................................................................... 95

4.1.2 Expression of beta-cell related transcription factors in beta-cell-specific Pax6 KO pancreata .96

4.1.3 Defective glucose-stimulated insulin secretion in beta-cell-specific Pax6 KO pancreata...........98

4.1.4 Defective proinsulin processing in beta-cell-specific Pax6 KO pancreata...............................99

4.1.5 Ratio of IAPP to insulin is changed in the beta-cell-specific Pax6 KO islets........................... 101

4.1.6 Long-term tracing of Pax6 KO beta-cells in relation to ghrelin expression............................. 101

4.1.7 Regeneration of beta-cells in the beta-cell-specific Pax6 KO islets ...................................... 105

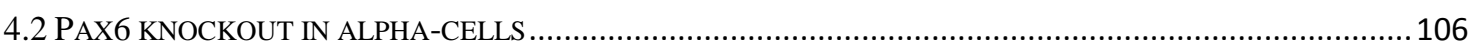

4.2.1 Loss of glucagon expression in Pax6 KO alpha-cells........................................................... 106

4.2.2 Ghrelin upregulation in Pax6 KO alpha-cells ............................................................... 106

4.2.3 7B2 upregulation in Pax6 KO alpha-cells.......................................................................... 108

4.2.4 Regeneration of alpha-cells in the alpha-cell-specific Pax6 KO islets.................................. 109

4.3 COMPARISON OF PAX6 KO IN ALPHA- AND BETA-CELLS.......................................................... 110

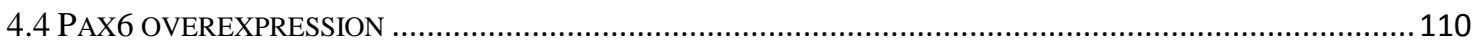

4.5 COMPARISON OF THE PAX6 PANCREATIC PHENOTYPE IN RELATION TO PAX6 FUNCTION IN BRAIN AND

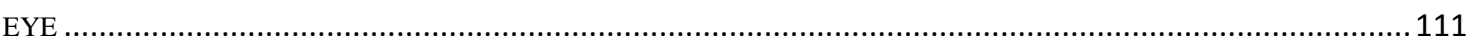

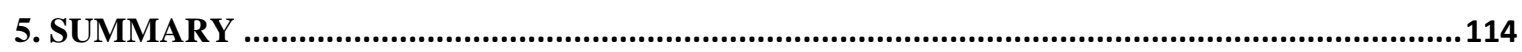

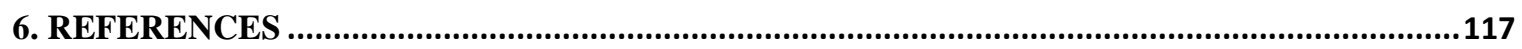




\section{List of Figures}

Figure 1.1: The pancreas consists of three main cell types: acinar, duct, and endocrine

Figure 1.2: Early development of pancreas. Induction of dorsal and ventral bud occurs through different pathways...................................... 9

Figure 1.3: Pax6 gene and protein....................................... 20

Figure 3.1: Lineage tracing with R26-YFP reporter mouse line.................. 43

Figure 3.2: Beta-cell-specific ablation of Pax6............................. 44

Figure 3.3: Inverse relationship of ghrelin and insullin expression in the pancreata of beta-cell-specific Pax6 KO mice.

Figure 3.4: Gradual increase in the population of ghrelin expressing cells in the islets of beta-cell-specific Pax6 KO mice.

Figure 3.5: Gradual decrease in the population of insulin expressing cells in the islets of beta-cell-specific Pax6 KO mice.

Figure 3.6: Changes in the glucagon ${ }^{+}$, somatostatin ${ }^{+}$, and $\mathrm{PP}^{+}$cell population in the islets of beta-cell-specific Pax6 KO mice.

Figure 3.7: Expression of beta-cell related transcription factors in the islets of beta-cell-specific Pax6 KO mice............................................ 50

Figure 3.8: Expression of Rfx6 in the islets of beta-cell-specific Pax6 KO mice.... 51

Figure 3.9: Expression of beta-cell related transcription factors in the pancreata of beta-cell-specific Pax6 KO mice. . .

Figure 3.10: Expression of Glut2 in the islets of beta-cell-specific Pax6 KO mice.. 53

Figure 3.11: Loss of Glut2 expression due to direct and/or indirect effect of Pax6 ablation in the islets of beta-cell-specific Pax6 KO mice. ...

Figure 3.12: Expression of GLP-1 receptor in the islets of beta-cell-specific Pax6 KO mice

Figure 3.13: Expression of PC1/3 and PC2 in the islets of beta-cell-specific Pax6 KO mice 56

Figure 3.14: Expression of C-peptide in the islets of beta-cell-specific Pax6 KO mice

Figure 3.15: Expression of proSAAS in the islets of beta-cell-specific Pax6 KO mice 
Figure 3.16: Expression of 7B2 in the islets of beta-cell-specific Pax6 KO mice... 59

Figure 3.17: Gradual increase in the population of proSAAS and 7B2 expressing cells in the islets of beta-cell-specific Pax6 KO mice 60

Figure 3.18: Co-expression of ghrelin with insulin in the islets of beta-cell-specific Pax6 KO mice.

Figure 3.19: Expression of beta-cell related transcription factors in the ghrelin ${ }^{+}$ cell population of the beta-cell-specific Pax6 KO islets.

Figure 3.20: Co-expression of ghrelin with beta-cell related factors in the islets of beta-cell-specific Pax6 KO mice.

Figure 3.21: Ghrelin ${ }^{+}$cells originate from beta-cells in the beta-cell-specific Pax6 KO islets. 65

Figure 3.22: Changes in the ghrelin expressing cell population over time in the islets of beta-cell-specific Pax6 KO mice.

Figure 3.23: Pax6-deficient beta-cells do not proliferate.

Figure 3.24: Pax6 does not bind to Glut2 and ghrelin promoters. 70

Figure 3.25: Development of diabetes in the beta-cell-specific Pax6 KO mice......71

Figure 3.26: Beta-cell regeneration in the beta-cell-specific Pax6 KO mice. 72

Figure 3.27: Proliferation in the regenerating population of beta-cells in the betacell-specific Pax6 KO islets. ...

Figure 3.28: Beta-cell regeneration in the beta-cell-specific Pax6 KO islets may occur via transdifferentiation and/or neogenesis.............................. 75

Figure 3.29: Alpha-cell-specific ablation of Pax6...........................77

Figure 3.30: A mixed population of ghrelin and glucagon positive cells at P0......78

Figure 3.31: Ghrelin ${ }^{+}$cell population is increased in the adult alpha-cell-specific Pax6 KO islets. 79

Figure 3.32: Ghrelin ${ }^{+}$cells originate from alpha-cells in the alpha-cell-specific Pax6 KO islets.

Figure 3.33: Expression of alpha-cell related transcription factors in the ghrelin ${ }^{+}$ cells of the alpha-cell-specific Pax6 KO islets. ...

Figure 3.34: Expression of alpha-cell-lineage determinant Arx in the ghrelin ${ }^{+}$cells of the alpha-cell-specific Pax6 KO islets. . .83

Figure 3.35: Alpha-cell regeneration in the alpha-cell-specific Pax6 KO islets. 
Figure 3.36: Expression of 7B2 and PC2 in the alpha-cell-specific Pax6 KO islets

Figure 3.37: Construct used to generate conditional Pax6-overexpressing (Pax6-

$O E)$ transgenic mice.

Figure 3.38: Pax6 overexpression under $P d x 1$, insulin, and glucagon expression domains.

Figure 3.39: Pancreatic hypoplasia in Pax6OE; Pdx1-Cre mice. . .90

Figure 3.40: Changes in the glucagon ${ }^{+}$, ghrelin ${ }^{+}$, and $\mathrm{PP}^{+}$cell population in the pancreata of Pax6OE; Pdx1-Cre mice. 90

Figure 3.41: Expression of beta-cell related factors in Pax6OE; Pdx1-Cre islets....91

Figure 3.42: Pax6OE; Ins-Cre islets do not show any change in the beta-cell population.

Figure 3.43: Changes in glucagon ${ }^{+}$and $\mathrm{PP}^{+}$cell pouplation in the islets of Pax6OE; Glu-Cre mice. .93

Figure 4.1: Schematic representation of the phenotypic change observed in Pax6deficient beta-cells. 103

Figure 4.2: Schematic representation of the phenotypic change observed in Pax6deficient alpha-cells. 107 


\section{List of Tables}

Table 2.1: List of primary antibodies used............................ 27

Table 2.2: List of secondary antibodies used...........................28

Table 2.3: List of qRT-PCR primers................................29

Table 2.4: List of PCR primers used in ChIP............................29

Table 2.5: List of PCR primers used in cloning.........................30

Table 2.6: List of PCR primers used for genotyping......................30

Table 2.7: List of mouse lines used......................................30

Table 2.8: Phosphate buffered saline (PBS) .......................... 31

Table 2.9: Luria-Bertani medium (LB medium)........................31 


\section{Abbreviations}

BMP Bone morphogenetic protein

BrdU Bromodeoxyuridine

ChIP Chromatin immunoprecipitation

DAPI 4',6-diamidino-2-phenylindole

dNTPs Deoxyribonucleoside triphosphates

E Embryonic day

FGF Fibroblast growth factor

GFP Green fluorescent protein

GLP Glucagon-like peptide

GSIS Glucose-stimulated insulin secretion

IAPP Islet amyloid polypeptide

KD Knockdown

KO Knockout

LB Luria-Bertani

MODY Maturity onset diabetes of the young

OE Overexpression

PBS Phosphate buffered saline

PC Prohormone convertase

PCR Polymerase chain reaction

PP Pancreatic polypeptide

qRT-PCR Quantitative reverse transcription polymerase chain reaction

RA Retinoic acid

RIP Rat insulin II promoter

R26 Rosa26

SEM Standard error of mean

Shh Sonic hedgehog

TUNEL Terminal deoxynucleotidyl transferase dUTP nick end labeling

X-Gal 5-bromo-4-chloro-indolyl- $\beta$-D-galactopyranoside

YFP Yellow fluorescent protein 


\section{Acknowledgements}

I am grateful to my supervisor, Prof. Dr. Ahmed Mansouri, for providing me the opportunity to work on this interesting project. His scientific guidance has helped me to explore the field of developmental biology and to pave my path to the doctoral degree. I am also thankful to my thesis committee members, Prof. Dr. Wolfgang Engel and Prof. Dr. Ralf Dressel, for their insightful comments and discussion during the meetings.

I would like to thank my colleagues in the Molecular Cell differentiation group, for their help and support in every possible way. In particular, I am thankful to Dr. Tamara Rabe, Mei-Chih Liao, and Farnaz Shamsi for the useful discussions and for sharing all the good and bad moments during this time period. I am also thankful to the BTL team for taking care of the mouse lines. I am thankful to all members of the department of Molecular Cell Biology, for creating a friendly working environment. I would also like to acknowledge Dr. Erik Meskauskas and Dr. Werner Albig from the coordination office of Molecular Medicine Program, for their administrative help.

I am greatly indebted to my parents, brother, and sister for their love, support, and prayers that have always helped me in my life. At the end, I would like to express my gratitude to my wife, Maria Rafeeq, for her love, care, and understanding that made this journey easier for me. 


\section{Introduction}

\subsection{Pancreas: morphology and function}

The pancreas is a lobulated gland that is located on the posterior abdominal wall behind the stomach. It is elongated in shape with a head, neck, body, and tail. The head lies towards duodenum, the body runs behind stomach, and its tail contacts the spleen. The pancreas is a gland with dual functions: an endocrine function that most importantly involves the maintenance of glucose homeostasis with the help of peptide hormones released into the blood stream and an exocrine function mediated through the release of digestive enzymes into the small intestine (duodenum), where they aid in the digestion process. Accordingly, the pancreas is comprised of an endocrine compartment and an exocrine compartment. Endocrine portion of the pancreas is arranged into globular structures named as the islets of Langerhans. Each islet is further composed of five different cell types: alpha, beta, delta, PP (pancreatic polypeptide), and epsilon that secrete peptide hormones glucagon, insulin, somatostatin, pancreatic polypeptide, and ghrelin, respectively (Figure 1.1). Exocrine portion of the pancreas is comprised of acinar cells, that secrete digestive enzymes, and duct cells that form ducts to collect these secretions and empty them into the duodenum along with their own secretion comprised of bicorbonate and mucins (Cleaver and Melton, 2005; Pan and Wright, 2011).

\subsection{Endocrine pancreas}

Islet of Langerhan is the functional unit of the endocrine pancreas. As mentioned above, each islet is composed of five hormone producing cell types. In mouse islets, beta-cells are mainly arranged at the centre while other cell types are arranged at the periphery. Human islets, on the contrary, possess a mixed architecture. Composition of individual cell types in each islet is also more homogeneous in mouse islets compared to human islets (Brissova et al., 2005; Jeon et al., 2009). Below is a brief description of each endocrine cell type and the relative hormone produced.

\subsubsection{Beta-cells}

Beta-cell is the most abundant cell type in the islet, constituting 61 to $88 \%$ of the mouse islets (Brissova et al., 2005). Beta-cells produce the peptide hormone insulin. Insulin is 


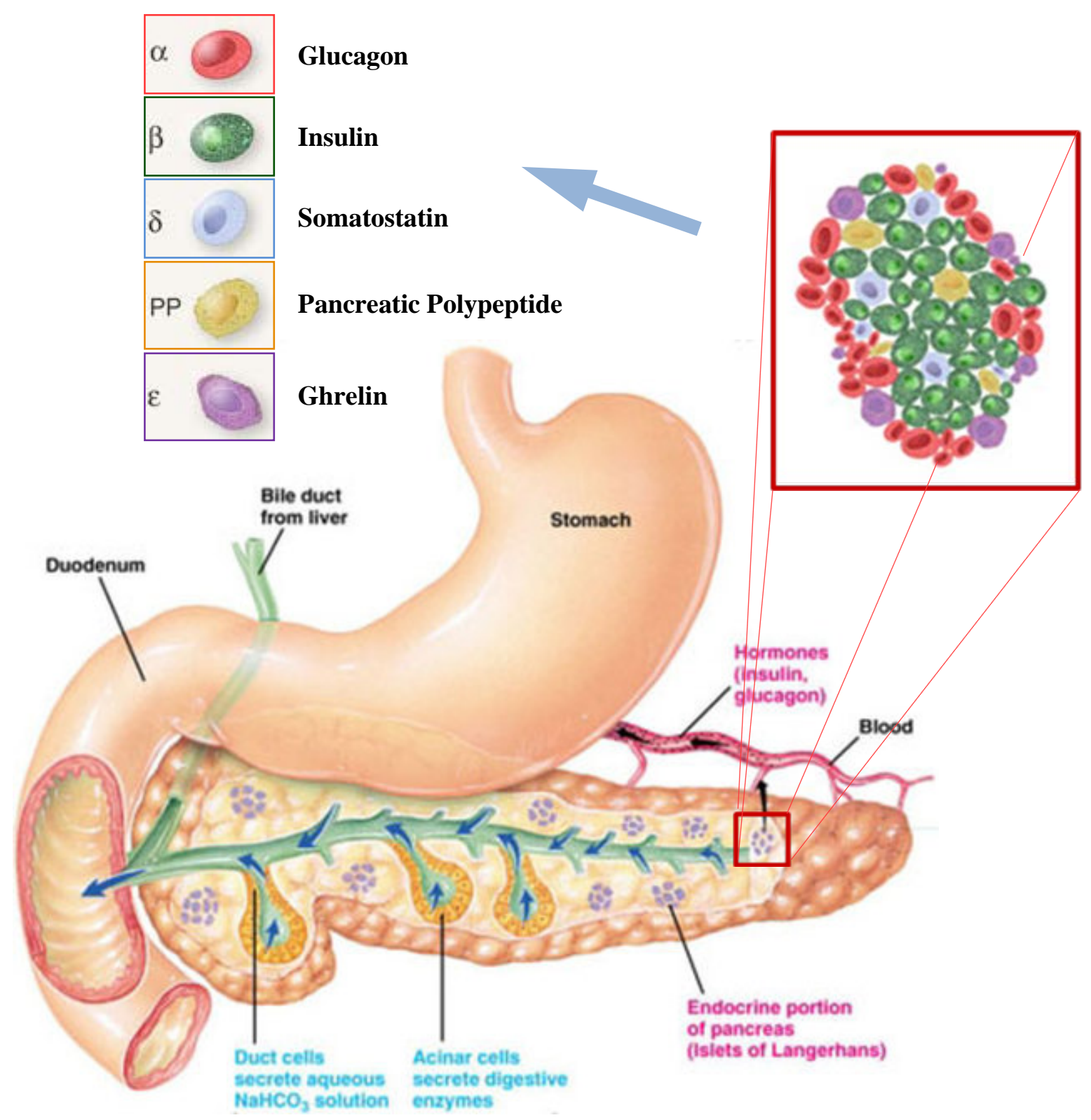

Figure 1.1: The pancreas consists of three main cell types: acinar, duct, and endocrine. Endocrine cells are clustered in the form of islets. Each islet is composed of five different hormone producing cell types. Modified from:

http://www.olivelab.org/the-pancreas-overview.html http://www.betacell.org/content/articleview/article_id/13?aid=13 
synthesized as a prohormone that consists of two chains A and B, and a middle portion called as the connecting peptide (C-peptide). This proinsulin is then cleaved by prohormone convertases (PC1/3 and 2) and carboxypeptidase $\mathrm{H}$ to generate the mature hormone. During this cleavage process C-peptide is released as a by-product at an equimolar ratio to insulin (Goodge and Hutton, 2000; Vasic and Walcher, 2012). Insulin is secreted from the beta-cells in response to glucose stimulation and exerts many anabolic effects in the body. It promotes glucose uptake and oxidation, stimulates glycogen synthesis, and inhibits gluconeogenesis. Additionally, it enhances the protein as well as fatty acid synthesis. Insulin action is mediated through insulin receptor (Najjar, 2003). Insulin knockout (KO) mice show growth retardation. They develop hyperglycemia and die within first two days after birth (Duvillié et al., 1997). Similarly, insulin receptor knockout mice also develop hyperglycemia and die early after birth (Kitamura et al., 2003). In humans, insulin gene mutations can cause maturity-onset diabetes of the young type 10 (MODY10) (Molven et al., 2008).

\subsubsection{Alpha-cells}

Alpha-cells constitute the second largest population in the islet, making 9 to $31 \%$ of the mouse islets (Brissova et al., 2005). Alpha-cells produce the hormone glucagon. Glucagon is also synthesized as a prohormone that is processed by prohormone convertase 2 (PC2) in alpha-cells to generate the mature glucagon. Proglucagon is also expressed in the intestinal $\mathrm{L}$ cells where it is differently processed by prohormone convertase 1/3 (PC1/3) to generate two other peptide hormones, glucagon-like peptide 1 and 2 (GLP-1 and 2), instead of glucagon (Wideman and Kieffer, 2009). Glucagon is released from alpha-cells in response to hypoglycemia and mainly acts in opposite to insulin. It promotes glycogenolysis and gluconeogenesis, while it inhibits the glycogen synthesis and glycolysis (Bansal and Wang, 2008). Glucagon action is mediated through the glucagon receptor. Glucagon receptor knockout mice exhibit hypoglycemia and hyperplasia of alpha-cells (Gelling et al., 2003). A similar phenotype is also observed in PC2 knockout mice that have defective proglucagon processing (Furuta et al., 1997).

\subsubsection{Delta-Cells}

Nearly 5 to $10 \%$ of the islet is composed of delta-cells (Hauge-Evans et al., 2009). Deltacells produce the hormone somatostatin. Biological actions of somatostatin are mainly of 
inhibitory nature. Both insulin and glucagon can induce the release of somatostatin which, in turn, inhibits the release of insulin and glucagon. Somatostatin can also inhibit the exocrine secretions, and the release of ghrelin and pancreatic polypeptide (Kojima et al., 2007; Chanclón et al., 2012). In somatostatin knockout mice the development of endocrine and exocrine pancreas is completely normal. These mice, however, show increased insulin and glucagon secretion after nutrient stimulation (Hauge-Evans et al., 2009).

\subsubsection{PP-cells}

PP-cells constitute about $6 \%$ of the islet cells (Adeghate and Ponery, 2003). These cells produce the hormone pancreatic polypeptide. Pancreatic polypeptide release is stimulated by food ingestion. It suppresses food intake and inhibits gastric emptying as well as the biliary and pancreatic exocrine secretion. Pancreatic polypeptide may also have a role in increasing the hepatic insulin sensitivity. As expected from its actions, pancreatic polypeptide overexpressing mice are thin and show decreased intake of food and gastric emptying (Banerjee and Onyuksel, 2012; Kojima et al., 2007).

\subsubsection{Epsilon-cells}

Epsilon-cells constitute a very minute population of endocrine cells in the adult islet. These cells produce the hormone ghrelin (Prado et al., 2004). However, ghrelin is also produced by some endocrine cells (P/D1 cells in humans and X/A like cells in rodents) in the stomach (Inui et al., 2004). Actually, pancreas is the major source of ghrelin during fetal life while stomach produces nearly all of the circulating ghrelin during adult life. Accordingly, the number of epsilon-cells is higher in the developing fetal pancreas and starts to decrease in the early postnatal life. In adult pancreas epsilon-cells are almost undetectable (Wierup et al., 2004; Chanoine and Wong, 2004).

Ghrelin is an orexigenic peptide that is secreted in response to fasting (Inui et al., 2004). Apart from its role in food intake regulation, ghrelin is involved in stimulating the growth hormone release from pituitary (Kojima et al., 1999). It also acts to promote cell proliferation and survival, and to reduce apoptosis (Granata et al., 2006, 2007). In the endocrine pancreas, ghrelin promotes the glucagon secretion and inhibits the secretion of insulin and somatostatin (Chanclón et al., 2012). 
Role of ghrelin in the developing pancreas is so far not clear. Epsilon-cell number is tremendously increased when some important pancreatic endocrine transcription factors are knocked out. These include Nkx2.2, Pax4, and Pax6 (Prado et al., 2004; Kordowich et al., 2011). However, in ghrelin knockout mice the pancreatic development is completely intact. Recently, Arnes et al. (2012) have shown by lineage tracing that ghrelin expressing cells are not terminally differentiated cells. Instead, they represent an intermediate progenitor population that would later give rise to a subset of alpha and PP-cells.

\subsection{Diabetes mellitus}

Diabetes mellitus is a metabolic disease identified by hyperglycemia i.e., the high blood glucose level. It is caused by insufficient insulin production from the beta-cells and/or due to insulin resistance i.e., the inability of the peripheral tissues to correctly respond to insulin. In either case, the result is an elevated blood glucose level. Acute symptoms caused by hyperglycemia include, increase in thirst and hunger, increase in urination, and loss of weight. It can further lead to ketoacidosis that can be fatal. Chronically, hyperglycemia can impair the function of different organs. There are two main types of diabetes, type 1 and type 2. Type 1 diabetes is caused by the cell-mediated autoimmune destruction of the beta-cells that eventually leads to nearly complete loss of insulin production. This form of diabetes is treated by providing external insulin, mostly in the form of injection. On the other hand, type 2 diabetes is initially caused by insulin resistance and a partial insulin deficiency. Accordingly, the treatment involves oral hypoglycemic agents, exercise, and diet changes in the beginning. Only if the insulin deficiency becomes severe at a later stage, the insulin therapy is recommended. Gestational diabetes is a form of type 2 diabetes that results from insufficient insulin production during pregnancy. Rarely, diabetes is also caused by monogenetic disorders (also called maturity onset diabetes of the young MODY). (Buchanan and Xiang, 2005; Stumvoll et al., 2005; Daneman, 2006; Slingerland, 2006)

( http://www.ncbi.nlm.nih.gov/pubmedhealth/PMH0002194/).

\subsection{Development of the pancreas}

During embryonic development of vertebrates, gastrulation results in the specification of three germ layers namely ectoderm, mesoderm, and endoderm (Arnold and Robertson, 
2009). The endoderm further undergoes patterning along the anteroposterior axis to generate foregut, midgut, and hindgut domains (Grapin-Botton and Melton, 2000). Two factors that play important roles at this initial step include: fibroblast growth factor 4 (FGF4) and retinoic acid (RA). FGF4 aids in posteriorising the endoderm by promoting the expression of posterior endodermal transcription factors Pdx1 and Cdx1/Cdx2 (Wells and Melton, 2000; Dessimoz et al., 2006). Retinoic acid is important for the initial anterior-posterior patterning as well as for the later pancreas development (Chen et al., 2004; Stafford et al., 2004, 2006; Bayha et al., 2009).

\subsubsection{Generation of pancreatic buds}

After early patterning, pancreas then develops from the foregut endoderm as two pancreatic evaginations. The dorsal pancreatic bud is first to appear as an outgrowth of the dorsal foregut at the duodenal region. This happens around embryonic day 9.5 (E9.5) in mouse and around gestational day 26 (G26d) in humans. Half a day later in mouse (E10) and six days later in humans (G32d) the ventral pancreatic bud appears as an evagination of the ventral foregut close to the hepatic/biliary region (Gittes, 2009). Dorsal and ventral pancreas development proceeds through different pathways. Dorsal pancreas first receives signals from the notochord, then from the dorsal aorta, and lastly from the pancreatic mesenchyme. Ventral pancreas receives signals from the cardiac mesoderm, lateral plate mesoderm, and the septum transversum mesenchyme (Pan and Wright, 2011).

\subsubsection{Genesis of dorsal pancreas}

In the beginning, prepancreatic dorsal endoderm is in contact with the notochord and receives permissive signals in the form of activin-betaB and FGF2 that may suppress Sonic hedgehog (Shh) in the endoderm to allow dorsal bud formation (Hebrok et al., 1998). Around E8 then the fusion of dorsal aortas moves the notochord away from the dorsal endoderm. At this time, the aortic endothelial cells may help to induce Pdx1 and Ptf1a expression in the dorsal endoderm. Furthermore, they also promote the survival of dorsal pancreatic mesenchyme. Subsequently, the pancreatic mesenchyme grows and envelops the epithelium. Further pancreatic development then depends on the signals from the mesenchyme. Pancreatic epithelium cultured in the absence of mesenchyme does not develop (Golosow and Grobstein, 1962). Later, it was found that the 
mesenchyme is mainly required for the acinar formation while islet formation is the default pathway in the absence of mesenchyme (Gittes et al., 1996). Detailed study further showed that mesenchymal-epithelial contact induces notch-signalling mediated hes1 expression in the epithelium that would repress $\mathrm{Ngn3}$ and promote acinar differentiation (Duvillie et al., 2006). Additionally, the FGF signalling from mesenchyme to epithelium promotes epithelial proliferation (Miralles et al., 1999; Elghazi et al., 2002).

\subsubsection{Genesis of ventral pancreas}

Ventral pancreas and liver seem to originate from a common progenitor population located on the ventral side of foregut endoderm (Deutsch et al., 2001). Cardiac mesoderm induces hepatic differentiation from ventral endoderm via FGF signaling (Gualdi et al., 1996; Jung et al., 1999). In the absence of this signal the pancreatic fate is followed that seems to be the "default pathway" for the ventral endoderm (Deutsch et al., 2001). Bone morphogenetic protein (BMP) signals from the septum transversum mesenchyme are also involved in inducing the liver fate at the expense of ventral pancreas (Rossi et al., 2001). Therefore, in order to form the ventral pancreas, a part of the ventral endoderm must escape the hepatogenic signals from the mesoderm. This escape is achieved by movement of a part of the endoderm away from the cardiac mesoderm. It has been shown that this tissue positioning is achieved via Hex gene that controls the proliferation rate and, therefore, the proper positioning of the leading edge of endoderm (Bort et al., 2004).

\subsubsection{Bud to gland formation}

The early dorsal and ventral pancreatic buds (at E10 in mouse) predominantly consist of multipotent pancreatic progenitor cells (MPC) interspersed with a couple of early endocrine cells, including mainly glucagon ${ }^{+}$cells. Some insulin-glucagon co-positive cells as well as ghrelin ${ }^{+}$cells are also evident at this time point. However, these early endocrine cells do not give rise to the mature endocrine cells and their function is not yet defined. Once generated, the dorsal and ventral bud epithelium proliferates and grows, this leads to elongation of the stalk region and branching at the bud tip. As the development continues, the gut tube rotates and brings the dorsal and ventral buds into close proximity. This leads to their fusion (around E12.5 in mouse and G6w in humans), generating a single definitive pancreas. By this time, along with the morphogenetic development, the pancreatic epithelium also undergoes a clear "tip-trunk" segregation. 
The tip region contains multipotential pancreatic cells (MPC), that would later give rise to the progenitors with an acinar fate, and the trunk region contains duct/endocrine bipotential progenitors (Figure 1.2) (Pan and Wright, 2011). It has been suggested that the final size of the adult mouse pancreas is determined by the number of the progenitor cells assigned to the developing pancreas between E9.5 to E12.5 (Stanger et al., 2007).

Around E13.5 in mouse, there is a sudden outburst of growth in the developing pancreas termed as the "secondary transition“. During this phase, there is a rapid differentiation and proliferation of acinar cells arising from the tip regions. At the same time, endocrine cells originate from the bipotential progenitors in the trunk regions. Alpha- and beta-cells are first to appear followed by the delta-cells a day later. Finally, PP-cells appear around E18. As the endocrine cells develop, they leave the trunk epithelium and start to cluster into islets (Figure 1.2). In the mouse, further arrangement and maturation of islets continues from late gestation to the first few weeks after birth. Beta-cells form the core of the islets in mouse, while other types of endocrine cells are arranged at the periphery. On the contrary, in mature human islets, alpha- and beta-cells are inter-mingled. Following the secondary transition, pancreatic epithelium continues to expand mainly via proliferation of acinar cells. Expansion of islet cell mass after birth is also predominantly achieved through self-duplication. However, once the mature islet cell mass is achieved the ability of endocrine cells (e.g., beta-cells) to divide is strongly reduced (Collombat et al., 2006; Pan and Wright, 2011).

\subsection{Role of transcription factors in developing and adult endocrine pancreas}

Gene knockout, overexpression, and misexpression studies in mice have generated a wealth of information on the role of many different transcription factors in the development and function of endocrine pancreas. In the following section, some of the important transcription factors are reviewed. The expression pattern of each transcription factor in the developing and adult pancreas is described, and the published knockout and/or overexpression phenotype is mentioned. In view of the thesis topic, the role of Pax6 is discussed in a separate section in greater detail. 


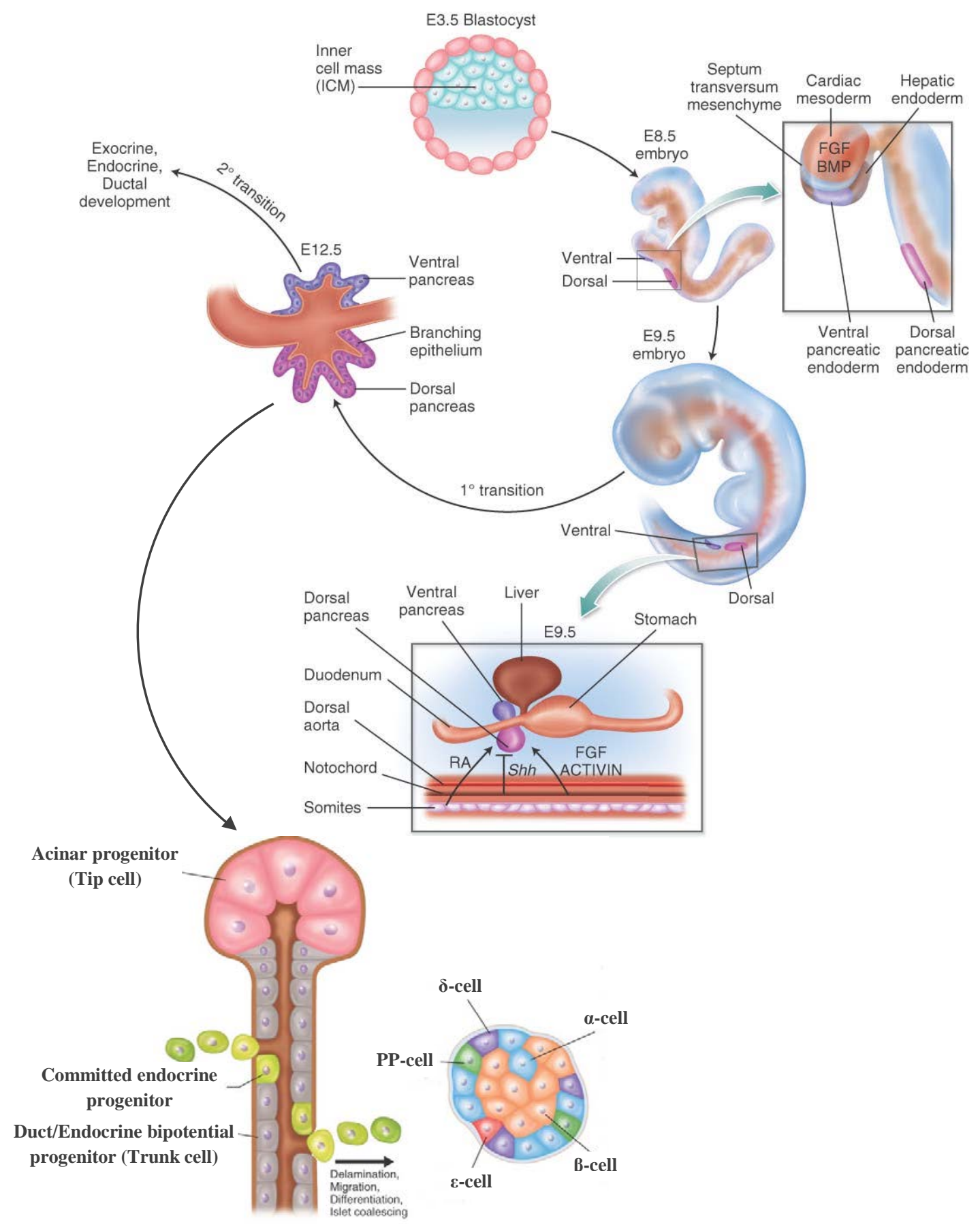

Figure 1.2: Early development of pancreas. Induction of dorsal and ventral bud occurs through different pathways. After the bud epithelium undergoes branching morphogenesis, the tip-trunk segregation becomes apparent. Tip gives rise to acinar cells and trunk contains bipotential progenitors for ductal and endocrine cells. Once a cell becomes committed to endocrine lineage, it leaves the ductal epithelium, undergoes further differentiation, and clusters with other endocrine cells to form an islet (modified from Mastracci and Sussel, 2012). 


\subsubsection{Hlxb9}

Hlxb9 expression is detectable in the developing mouse embryo around E8. At this stage, it is expressed in the whole endoderm destined to become pancreas as well as in the notochord. Around E9.5, the Hlxb9 expression forms a gradient with higher expression to the dorsal side and lower to the ventral side and the expression generally declines towards E12.5 (Sherwood et al., 2009). Later on, during the secondary transition, strong expression comes back and is limited to the $\mathrm{Pax}^{+}$endocrine precursors. Finally, Hlxb9 expression becomes restricted to the beta-cells in the adult pancreas (Harrison et al., 1999; Li et al., 1999). Apart from the pancreas, Hlxb9 expression is also detected in the developing esophagus, stomach, and small intestine (Li et al., 1999).

In Hlxb9 knockout mice, dorsal pancreatic bud shows complete agenesis, while the ventral bud development is apparently normal. However, the beta-cells originated from the ventral pancreas are not fully mature as they lack the expression of Glut2, the glucose transporter in beta-cells that plays an important role in the glucose-stimulated insulin secretion (GSIS). Furthermore, Hlxb9 knockout mice died at birth due to failure of the respiratory system (Thaler et al., 1999). On the other hand, over-expression of Hlxb9 in the entire $P d x 1$ domain disturbs the development of both exocrine and endocrine pancreas, and instead induces an intestinal-like differentiation program in the pancreatic anlage. These Hlxb9 overexpressing mice also die shortly after birth (Li and Edlund, 2001).

\subsubsection{Pdx1}

$P d x 1$ expression is first evident at E8.5 in the entire pancreatic-fated region of the endoderm. Around E10 to E11.5, its expression extends to the common bile duct, duodenum, and distal stomach (Jonsson et al., 1994, 1995; Offield et al., 1996). Moreover, lineage-tracing in the pancreas has confirmed that the duct, acinar, and endocrine cells are all generated from the $\mathrm{Pdx} 1^{+}$progenitors (Gu et al., 2002). $P d x 1$ expression undergoes dynamic changes during pancreas development. Initially, it is expressed in the entire epithelium at high levels. Then its expression is reduced as the cells are specified to the endocrine, duct, and acinar lineages (Jensen et al., 2000b; Gu et al., 2002; Hale et al., 2005). Subsequently, high level of $P d x 1$ expression is re-induced in the beta-cells and is required for their maturity and proper function (McFarlane et al., 
1994; Marshak et al., 1996). A low level of $P d x 1$ expression is maintained in the acinar, ductal, and other types of endocrine cells (Guz et al., 1995; Wu et al., 1997).

In $P d x 1$ knockout mice, initial budding of the dorsal pancreatic bud occurs but fails to develop further. Additionally, unlike Hlxb9 mutant mice there is no ventral pancreas development either (Jonsson et al., 1995; Offield et al., 1996). Moreover, it was found that the dorsal pancreatic rudiment in these mutants does contain the first-wave endocrine cells that fail to expand further. These knockout mice died in the first few days after birth due to defects in the pancreatic as well as other gastro-duodenal areas (Larsson et al., 1996; Offield et al., 1996). $P d x 1$ expression is critical not only in the beginning but also in the subsequent phases of pancreatic development as shown by the later studies where $P d x 1$ was depleted after the initial budding had occurred (Holland et al., 2002; Hale et al., 2005).

As mentioned before, unlike other endocrine cells, $P d x 1$ is highly expressed in the mature beta-cells. To uncover the role of Pdx1 in mature beta-cells, Ahlgren et al., (1998) generated knockout of $P d x 1$ in beta-cells alone. These mice were initially healthy but developed an overt-diabetic phenotype with age (at 3-5 months), showing the importance of Pdx1 in maintaining the functional identity of beta cells. Consistent with the mouse studies, PDX1 homozygous null mutation causes pancreatic agenesis (Stoffers et al., 1997a), while a heterozygous mutation causes diabetes (MODY4) in humans (Stoffers et al., 1997b).

To further delineate the role of $\mathrm{Pdx} 1$ in pancreatic development, an opposite approach was applied by over-expressing the $P d x 1$ in the whole pancreatic anlage (Using Ptf1a regulatory domain). This led to the pancreatic hypoplasia with severe defect in the acinar cells. Acinar cells adopted a duct like phenotype and their apoptosis was also increased (Miyatsuka et al., 2006). On the other hand, overexpression of $P d x 1$ in the endocrine progenitors (using $\mathrm{Ngn} 3$ regulatory domain) led to the reprogramming of alpha- to betacells (Yang et al., 2011).

\subsubsection{Pbx1}

At E10.5, Pbx1 expression is detected in the pancreatic epithelium and surrounding mesenchyme. Later in development, the expression is maintained in the mesenchyme and reduced in the epithelium. Finally, in the adult pancreas, $P b x 1$ is expressed in the acinar, ductal, and endocrine cells. $P b x 1$ knockout mice have pancreatic hypoplasia with a severe 
defect in both endocrine and exocrine cell differentiation. They die before birth during embryonic development (around E15 to E16). Pbx1 heterozygous mutants are initially healthy, however, they develop glucose intolerance and hypoinsulinemia with age. Furthermore, when $P b x 1$ heterozygous mutation is combined with a heterozygous $P d x 1$ mutation the resultant phenotype is much more drastic as the mice develop age-related overt diabetes mellitus (Kim et al., 2002). Indeed, Pbx1 and Pdx1 have been shown to interact with each other (Dutta et al., 2001; Swift et al., 1998). In acinar cells, the elastase I expression is activated by a regulatory complex in which Pdx1 interacts with Pbx1 (Liu et al., 2001).

\subsubsection{Ptf1a}

Ptf1a is a member of the heterotrimeric Pancreas Transcription Factor 1 complex (PTF1). This complex comprises of three proteins: Ptf1a (or p48), E2A (or p75), and RBP-J/RBPJL (Rose et al., 1994; Krapp et al., 1996). Ptf1a expression starts around E9.5 in both the dorsal and ventral pancreatic foregut endoderm (Krapp et al., 1998). As the development proceeds, expression is reduced in the endocrine lineage and is maintained in the developing and mature acinar cells of the pancreas. As confirmed by the lineage-tracing in mice, pancreatic progenitors expressing Ptf1a in the initial stage of development give rise to acinar cells, nearly all of the ductal, and most of the endocrine cells (Kawaguchi et al., 2002). In Ptf1a knockout mice, pancreatic agenesis is evident with almost no ventral bud and a very rudimentary dorsal bud that does not develop beyond the initial outgrowth. These mice die within first few hours after birth. As expected, acinar cells do not develop in the Ptf1a knockouts, however, a reduced number of endocrine cells do develop including a significant number of relatively mature beta-cells (Krapp et al., 1998; Burlison et al., 2008). Therefore, it appears that Ptf1a is not required for the endocrine cell specification but is important for the initial allocation and amplification of pancreatic progenitors that would later give rise to the endocrine population. This idea is supported by the fact that Ptf1a misexpression together with $P d x 1$ can convert duodenum into pancreas with an intact acinar to endocrine cell ratio (Afelik et al., 2006). In accordance to the mouse studies, PTF1a null mutation causes permanent neonatal diabetes mellitus in humans due to pancreatic agenesis (Sellick et al., 2004). 


\subsubsection{Isl1}

Isl1 expression is first detected in the dorsal pancreatic epithelium and the surrounding mesenchyme around E9 to E9.5. Later, it is expressed in the endocrine cells during development as well as in the adult pancreas. Isl1 knockout mice do not develop dorsal pancreatic mesenchyme and dorsal pancreatic bud. As they die at E9.5 before the ventral bud emergence, it is not possible to investigate the effect on ventral bud development (Ahlgren et al., 1997). To analyze the role of Isl1 in the later stages of development, Du et al. (2009) generated pancreas-specific Isl1 knockout mice. These mice are born alive but develop hyperglycemia from early postnatal life onwards that finally leads to their death between 3 to 8 weeks of age. In the mutant pancreata, the number of endocrine cells is significantly declined due to reduced proliferation and increased apoptosis. Moreover, the beta-cells present in the mutant pancreata are not fully mature as they lack the expression of MafA. Therefore, Isl1 is initially required for the maintenance of pancreatic mesenchyme and subsequently for the maturation and expansion of endocrine cells.

\subsubsection{Sox 9}

Sox9 is expressed around E10.5 in the early pancreatic progenitors. Lineage-tracing experiments have confirmed that these Sox9 expressing progenitors give rise to the acinar, ductal, and endocrine cells of the pancreas (Akiyama et al., 2005). In the course of secondary transition, Sox9 expression is confined to the duct/endocrine bipotential progenitors in the developing epithelium. Finally, Sox9 expression is then restricted to the ductal epithelium of the adult pancreas (Lynn et al., 2007). Pancreas-specific Sox9 knockout mice show hypoplasia of dorsal as well as ventral pancreatic bud and die within first few days after birth. Moreover, as a cause behind this phenotype, it was found that Sox9 is required for the maintenance of pancreatic progenitors by restricting their premature differentiation, as well as by promoting their survival through increased proliferation and decreased apoptosis (Seymour et al., 2007). In addition to this, Sox9 has been found to regulate the expression of $\mathrm{Ngn3}$, and therefore, may play an important role in initiating the endocrine differentiation program (Lynn et al., 2007). 


\subsubsection{Ngn3}

Ngn3 is considered as a master regulator in the pancreatic endocrine differentiation program. Ngn3 expression is first detected in the early endocrine progenitors around E9.5. Later, the expression increases as the endocrine progenitors expand, and peaks around E15.5. From there on, it decreases and finally becomes nearly undetectable in the late embryonic and adult pancreas (Gradwohl et al., 2000; Schwitzgebel et al., 2000). In fact the expression of Ngn3 in the endocrine progenitors is transient and occurs only in the undifferentiated progenitors. Ngn3 directly activates many of the endocrine specific transcription factors, while it represses its own promoter activity (Huang et al., 2000; Smith et al., 2003, 2004, 2010; Mellitzer et al., 2006). Once the endocrine differentiation program has been initiated in a cell, the expression of $\mathrm{Ngn3}$ is then repressed (Jensen et al., 2000; Smith et al., 2004). Accordingly, the Ngn3 expression is biphasic and corresponds to the two phases of endocrine cell generation (primary and secondary). As more endocrine cells are generated in the secondary phase, the Ngn3 expression also peaks around this time (Villasenor et al., 2008).

Ngn3 knockout mice do not develop any of the endocrine cell types and die within first few days after birth (Gradwohl et al., 2000). Lineage-tracing analysis also confirms the generation of all the endocrine cell types from the $\mathrm{Ngn3}$ expressing progenitors (Gu et al., 2002). On the other hand, overexpression of $N g n 3$ under $P d x 1$ domain directs the entire progenitor pool to differentiate into endocrine tissue that consists of predominantly alpha-cells (Apelqvist et al., 1999). Later on, Johansson et al. (2007) found that the ability of Ngn3 to induce various endocrine cell types is associated with the specific developmental time-point. They used Ngn3-addback strategy to induce Ngn3 expression at various time points of pancreatic development in mice that are Ngn3 deficient. It was found that Ngn3 activation around E9 generates alpha-cells alone, activation around E11.5 generates alpha, beta, and gamma-cells, and activation around E14.5 generates all four types of endocrine cells. Apart from the timing, the level of Ngn3 expression is also very important. Endocrine progenitors with a high Ngn3 expression are truly committed to the endocrine fate, while the progenitors with low Ngn3 expression can opt for other pancreatic fates as well (Wang et al., 2010). Therefore, progenitors with low Ngn3 expression may represent an intermediate state where the cell has still a choice to move in either direction. 


\subsubsection{Rfx6}

$R f x 6$ is broadly expressed in the early gut endoderm. At E9, its expression is detected in the dorsal pancreatic bud. Around this time, many Rfx6 positive cells co-express $P d x 1$. Later around E10-12.5, Rfx6 expression gets more restricted to the differentiating endocrine cells and is excluded from $P d x 1$ or Ptf1a expressing cells. During the secondary transition, many of the Rfx6 positive cells co-express Ngn3. Furthermore, in $\mathrm{Ngn3}^{-/-}$mice the expression of $R f x 6$ is lost indicating that Rfx6 is Ngn3-dependent transcription factor in the pancreas. Finally, in the adult pancreas, $R f x 6$ is expressed only in the endocrine cells of the islet. Rfx6 plays important role in the endocrine pancreas development as in the $R f x 6$ knockout mice, pancreatic endocrine cells do not develop except for the PP producing cells (Smith et al., 2010; Soyer et al., 2010).

\subsubsection{NeuroD}

NeuroD expression is first detectable in the endocrine committed cells of the early pancreas around E9.0 (Huang et al., 2000). Later in development, it continues to express in most of the endocrine cell population. Finally, as the islets mature in the adult pancreas, its expression becomes restricted to only beta-cells (Itkin-Ansari et al., 2005). NeuroD is one of the direct downstream targets of Ngn3 (Gradwohl et al., 2000; Huang et al., 2000; Jensen et al., 2000) and an important mediator in the induction of endocrine differentiation program (Gasa et al., 2008). This is supported by the fact that both Ngn3 and NeuroD can activate many of the same target genes (Gasa et al., 2008).

In contrast to Ngn3 knockout mice, NeuroD knockout mice can develop all types of endocrine cells. However, the number of endocrine cells is significantly reduced as they fail to expand after initial differentiation. Therefore, NeuroD knockout mice also develop hyperglycemia and die within first few days after birth (Naya et al., 1997). To understand the role of NeuroD in beta-cells, Gu et al., (2011) generated beta-cell-specific NeuroD knockout mice. These mice are slightly hyperglycemic and develop severe glucose intolerance due to immature beta-cell function. In humans, NEUROD1 heterozygous mutations are associated with maturity onset diabetes of the young type 6 (MODY6), and homozygous null mutations can cause permanent neonatal diabetes (Malecki et al., 1999; Rubio-Cabezas et al., 2010). 


\subsubsection{Nkx2.2}

$N k x 2.2$ expression begins in the early pancreatic epithelium around E9.0. Initially, it is expressed in most of the epithelial cells and later becomes gradually confined to the endocrine progenitors. $N k x 2.2$ expression then persists in beta-cells, and in a subset of alpha- and PP-cells. Nkx2.2 is not expressed in either developing or mature delta-cells (Sussel et al., 1998; Jørgensen et al., 2007). Accordingly, in Nkx2.2 knockout mice, betacells are lost, alpha- and PP-cells are reduced in number, and delta-cells are not changed. These mice develop hyperglycemia and die shortly after birth (Sussel et al., 1998). Later, it was found that the lost endocrine cells, in $N k x 2.2$ mutant mice, are replaced by epsiloncells expressing ghrelin (Prado et al., 2004). $N k x 2.2$ inactivation in pancreas alone produces a similar phenotype (Mastracci et al., 2013). A compound deficiency of $N k x 2.2$ and Arx leads to upregulation of ghrelin ${ }^{+}$cells that co-express somatostatin (Kordowich et al., 2011; Mastracci et al., 2011). Nkx2.2 can function both as an activator and a repressor of transcription in the endocrine pancreas depending on the specific cell type or the timepoint of development (Raum et al., 2006; Doyle et al., 2007; Papizan et al., 2011). For example, Nkx2.2 can activate MafA expression that is required for the beta-cell maturity (Raum et al., 2006) and represses Arx expression in beta-cells to maintain their identity (Papizan et al., 2011). On the other hand, Nkx2.2 repressor function is also required for alpha-cell differentiation (Doyle et al., 2007). It has been suggested that Nkx2.2 repressor activity is mainly required during embryonic development of the pancreas, while activator function is more important in mature beta-cells (Doyle et al., 2007; Doyle and Sussel, 2007).

\subsubsection{Nkx6.1}

Nkx6.1 expression is first detected in few cells of the early pancreatic epithelium around E9. Around E10.5, the expression is detected in most of the pancreatic epithelium. Later, the expression becomes restricted to endocrine progenitors and then to beta-cells alone (Sander et al., 2000; Jørgensen et al., 2007). In mature islets, $N k x 6.1$ is expressed only in the beta-cells. In Nkx6.1 knockout mice, beta-cells are significantly reduced in number but other endocrine cell types are not affected. This reduction arises from inefficient betacell neogenesis during secondary transition as the earlier population of beta-cells is not affected. Furthermore, analysis of $N k x 6.1 / N k x 2.2$ double knockout shows a phenotype 
similar to $N k x 2.2$ single knockout. Accordingly, $N k x 6.1$ expression is lost in $N k x 2.2$ knockout but $N k x 2.2$ expression is maintained in $N k x 6.1$ knockout pancreata. It is, therefore, suggested that Nkx6.1 acts downstream of Nkx2.2 (Sander et al., 2000). Inactivation of $N k x 6.1$ in endocrine precursors or beta-cells favors non-beta endocrine cell lineages at the expense of beta-cells while overexpression of $N k x 6.1$ in endocrine precursors selectively favors beta-cell fate (Schaffer et al., 2013). Therefore, Nkx6.1 is required for beta-cell specification and maintenance of beta-cell fate.

\subsubsection{Pax4 and Arx}

At E9.5, Arx expression is detectable in the pancreatic epithelium. During further development, Arx continues to express in the endocrine progenitors and then in the endocrine cells (mainly alpha-cells). In the adult pancreas, Arx is expressed in the alphaand PP-cells (Collombat et al., 2003, 2007). Pax4 expression also starts in the early pancreas at E9.5. Pax4 expression then increases and peaks around the time of secondary transition when majority of the endocrine cells are generated. Later, its expression is reduced to nearly undetectable levels and is restricted to beta-cells (Sosa-Pineda et al., 1997; Smith et al., 1999).

Arx and Pax4 are important for the early lineage specification in the endocrine pancreas and an opposing link has been described between them. In Arx knockout mice, alpha-cells fail to develop, beta- and delta-cells are increased in number, and PP-cells are not affected (Collombat et al., 2003). On the other hand, in Pax4 knockout mice, betaand delta-cells do not develop while alpha-cell numbers are increased (Sosa-Pineda et al., 1997). As expected from these phenotypes, in the Arx/Pax4 double knockout mice, both alpha- and beta-cells fail to develop but surprisingly the number of delta-cells is highly increased (Collombat et al., 2005). Furthermore, misexpression of Arx in the mature betacells can convert them to alpha-cells, and that of Pax4 in alpha-cells can convert them to beta-cells (Collombat et al., 2007, 2009). These studies establish the essential role of Arx in alpha-cell specification, and that of Pax4 in beta-cell specification.

\subsubsection{MafA and MafB}

$M a f B$ expression is detectable in the early pancreatic epithelium around E10.5. At this time, most of the MafB expression is observed in alpha-cells. Subsequently, during secondary transition, $M a f B$ is expressed in some endocrine progenitors, in most of the 
alpha- and many of the beta-cells. Finally, in adult pancreas, MafB expression is restricted to alpha-cells only (Artner et al., 2006; Nishimura et al., 2006). MafB knockout mice die at birth due to respiratory problems. Pancreatic analysis shows that alpha- and beta-cells are significantly reduced in number, while PP- and delta-cells are not affected in these mice. As compared to wild type mice, the total number of endocrine cells is also not changed. Therefore, the defect seems to be with the maturation of cells rather than their specification. Accordingly, MafB can bind to the promoters of glucagon, and insulin genes and activates their transcription. MafB may also activate the expression of other factors required for beta-cell maturity, including MafA, $P d x 1$, and Glut2 (Artner et al., 2007). Thus, MafB plays an important role in the development of mature alpha- and betacells.

In the developing pancreas, MafA expression starts later than MafB and is normally restricted to beta-cells. Around E12.5, few MafA positive beta-cells are visible. During secondary transition, the number of beta-cells expressing MafA increases (Nishimura et al., 2006). Finally, in the adult pancreas MafA is expressed in all of the beta-cells and not in other endocrine cells (Zhang et al., 2005). MafA knockout mice have normal pancreas development and appear healthy at birth. However, they develop glucose intolerance and diabetes mellitus with increasing age. Islet architecture, Insulin synthesis, and glucose stimulated insulin secretion is also affected in these mice (Zhang et al., 2005). Hence, MafA plays an important role in the functional maturity of beta-cells.

\subsection{Paired box genes}

The paired box gene family encodes nuclear transcription factors. It comprises of nine members that are characterized by the presence of a highly conserved paired box domain. This domain is named after the Drosophila gene Paired (Prd) where it was first identified. These nine members are placed into four subgroups based on the presence or absence of an octapeptide, and the presence of a complete, truncated or no homeodomain. Apart from the Paired domain and homeodomain that serve the purpose of DNA binding, all Pax proteins contain a C-terminal transactivation domain that is rich in Proline, Serine, and Threonine. Pax genes play an important role in the development and function of many different tissues. Therefore, mutations causing impairment in their function can lead to serious medical ailments (Buckingham and Relaix, 2007; Wang et al., 2008). 


\subsection{Paired box gene 6 (Pax6)}

Pax6 belonging to the group IV of Pax gene family is a highly conserved transcription factor among different animal species (Callaerts et al., 1997). The vertebrate Pax6 gene is organized into 16 exons that are numbered from 0 to 13 with two in between named alpha and 5a. Pax6 gene is transcribed from three different promoters: P0, P1, and P $\alpha$ (Figure 1.3). Mammalian Pax6 protein exists in three isoforms: Pax6 canonical, Pax6 5a, and Pax6 pairedless. These three isoforms are generated either by the selection of different promoters or by alternative splicing. Transcription from P0 and P1 promoters generate two different mRNAs that are translated into identical proteins because of the common translation start site. However, transcription from these two promoters shows different spatio-temporal patterns during embryonic development. The function of two different promoters for the expression of identical protein is not clear. However, the presence of different regulatory elements at each promoter may provide differential expression levels in different tissues or in the same tissue at different time points of development. Transcript initiated from P0 and P1 promoter is alternatively spliced to generate either Pax6 canonical or Pax6 5a isoform. Pax6 5a contains an additional exon (5a) that leads to a 14 amino acid insertion in the paired domain and changes the DNA binding properties of the protein. Third promoter Palpha is located within the intron between exon 4 and 5; and transcription from this promoter generates a Pax6 protein without paired domain (Shaham et al., 2012).

\subsection{Pax6 in mammalian development}

Initially, the role of Pax6 in development has been suggested by its expression in the developing tissues and by analysis of the phenotypes associated with naturally occurring Pax6 mutations. Later on, the generation of Pax6 knockout and more importantly the tissue specific Pax6 knockout mice has helped to understand the finer details of Pax6 role in the development and function of different tissues. Pax6 is expressed in the mouse eye, central nervous system (CNS), olfactory system, and pancreas (Walther and Gruss, 1991). Mice homozygous for Pax6 null mutation die early after birth. They lack eyes, and nasal cavities, and the development of CNS and endocrine pancreas is also disturbed (Hill et al., 1991; Ashery-Padan et al., 2004; Osumi et al., 2008), further confirming the role of Pax6 in the development of these organs. Below is a brief description of the Pax6 role in 
a)

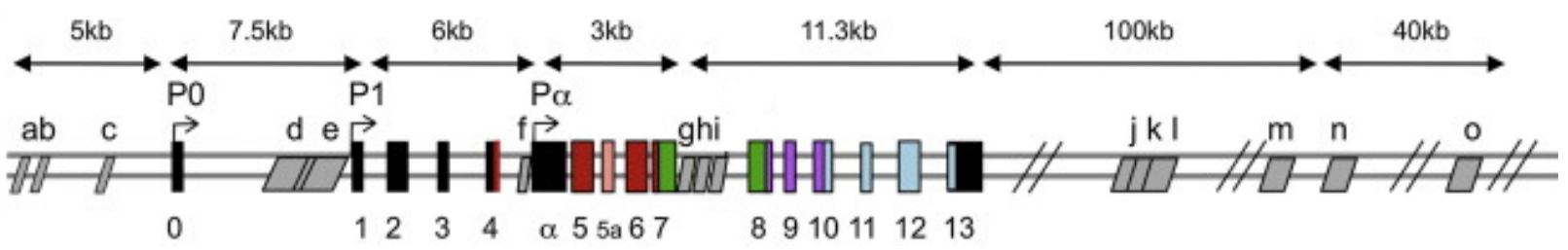

b)

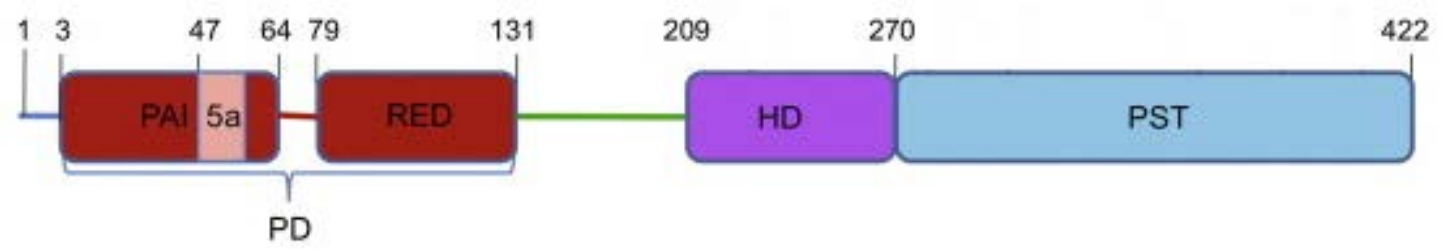

Figure 1.3: Pax6 gene and protein. (a) Pax6 gene structure. Colored boxes indicate coding exons and black boxes indicate non-coding exons. Grey boxes indicate various regulatory elements. (b) Pax6 protein domains. Note the additional 5a insertion in the paired domain that is not present in canonical isoform. $\mathrm{PD}=$ aired domain, $\mathrm{HD}=$ homeodomain, and PST=Proline-Serine-Threonine rich transactivation domain (adapted from Shaham et al., 2012). 
eye and brain development. Subsequently, the role of Pax6 in the endocrine pancreas development and function is discussed in greater detail.

\subsubsection{Pax6 in eye development}

Role of Pax6 in eye development was first to be identified and has been extensively studied. Aniridia is a human disease of the eye that is caused by heterozygous mutations in the PAX6 gene (Ton et al., 1991). In mouse, a heterozygous Pax6 mutation results in the "small eye" phenotype and homozygous Pax6 mutation leads to complete absence of eyes and is lethal in both mouse and human (Hill et al., 1991; Hodgson and Saunders, 1980). Therefore, a diploid dosage of Pax6 is essential for normal eye development. On the contrary, overexpression of Pax6 can also lead to severe eye defects (Schedl et al., 1996). Mammalian eye develops from three derivatives of ectodermal origin namely the neuroectoderm, head surface ectoderm, and the neural crest. Pax6 is expressed in the surface and neuroectodermal derivatives of the eye. It is required for the retinal neurogenesis, and for the specification of the ciliary body and iris progenitor pools. It is also required for the lens placode formation, for the lens vesicle detachment from the surface ectoderm, and for the lens fiber differentiation. Moreover, the corneal development is also disturbed in the Pax6 mutant mice (Shaham et al., 2012).

\subsubsection{Pax6 in brain development}

In the mouse embryo, Pax6 expression is first detectable around E8 in the neural plate. Subsequently, after neural tube regionalization, Pax6 expression is found in certain areas of the forebrain, midbrain, hindbrain, and the spinal cord (Walther and Gruss, 1991; Duan et al., 2013). Pax6 plays multiple roles in the development of central nervous system. It is required for the neural tube patterning, neuronal subtype specification, proper neuronal migration, and also for the neurogenesis itself. Appropriate dosage of Pax6 is required to control the proliferation of neural progenitor cells, the timing of cell cycle exit, and the differentiation into neurons (Osumi et al., 2008; Georgala et al., 2011). In the adult brain, Pax6 expression continues in the neurogenic niches as well as in certain subtypes of mature neurons. Therefore, Pax6 might be involved in maintaining the neuronal features of some neuronal subtypes (Duan et al., 2013). 


\subsubsection{Pax6 in Pancreas development}

Pax6 expression is first detected around E9 in the developing pancreatic epithelium. Around E10, it is detected in the early glucagon cells and in most of the Isl1 expressing cells in the pancreatic epithelium. Later, Pax6 expression is detected in the endocrine cells from the secondary transition. It then continues to express in alpha, beta, PP, and delta cells of the islet during later gestation as well as during adult life (Sander et al., 1997; St-Onge et al., 1997; Jensen et al., 2000a). Epsilon-cells, the fifth type of endocrine cells in the islet, may or may not express Pax6 (Kordowich et al., 2011). The expression pattern of Pax6 in developing and adult pancreas is suggestive of a vital role of Pax6 in the endocrine pancreas.

\subsubsection{Pax6 knockout in vivo studies}

In 1997, St-Onge et al. published the pancreatic phenotype of Pax6 KO mice. They found a reduction of endocrine cell population that was the most prominent among alpha-cells. Additionally, the normal islet architecture was disturbed in the mutant pancreata. Due to the fact that Pax6 KO mice die shortly after birth, it was not possible to analyze the role of Pax6 in adult Pancreas. In the same year, Sander et al. (1997) described a similar phenotype in $P a x 6^{\text {sey/sey }}$ mutant mice. Moreover, it was found that Pax6 can bind to insulin, glucagon, and somatostatin gene promoters and transactivate the expression of insulin and glucagon.

In an effort to analyze the role of Pax6 in postnatal pancreas development, AsheryPadan et al. (2004) used Cre-loxP system to generate the conditional inactivation of Pax6 in $P d x 1$ and Pax6 regulatory domains. The phenotype was similar to the classical Pax6 KO. Furthermore, they used Z/AP reporter strain to demonstrate that the overall endocrine area in the mutant pancreata was not reduced and, therefore, the islet cell neogenesis was not affected. Similar to the classical KO these conditional KO mice died in a few days after birth leaving the role of Pax6 in adult pancreatic function unresolved.

Later on, Heller et al. (2005) further analyzed Pax6 $6^{\text {sey/sey }}$ pancreata and found that the ghrelin positive cell population was 5 fold increased compared to the wild type. However, increase in ghrelin positive cell population has also been reported in Pax4 and Nkx2.2 KO pancreata (Prado et al., 2004). Since these three KO models die shortly after birth it was never possible to analyze the phenotype in adult pancreas. Analysis of ghrelin positive 
cells in the embryonic pancreas is further complicated due to the existence of these cells in the wild type pancreas as a separate population as well as being co-positive with alphacells. Therefore, the origin of ghrelin positive cells in the embryonic KO pancreas cannot be faithfully defined.

Among the endocrine cells, alpha-cells are the most severely affected ones in the Pax6 knockout mice. To understand the relative role of different Pax6 domains in the development of alpha-cells, Dames et al. (2010) analyzed the pancreata of Pax6 mutant mice that carried mutations in either paired domain, homeodomain, or transactivation domain of Pax6. It was found that the activity of paired domain is more important for the development of alpha-cells. On the other hand, mutation in the homeodomain results into least affected phenotype.

Recently, Hart et al. (2013) used tamoxifen inducible ubiquitous Cre line to knockout Pax6 in adult mice. They observed an overt diabetes and weight loss in knockout animals. Furthermore, the expression of insulin, glucagon, and somatostatin was reduced. In accordance with the previous studies they found an increased expression of ghrelin in the knockout pancreata.

The only in vivo study of Pax6 overexpression (OE) in mice resulted in diabetes due to apoptosis of beta-cells. In addition, overexpression under $P d x 1$ domain resulted in hypoplasia of exocrine pancreatic portion, and pancreatic tumors (Yamaoka et al., 2000).

\subsubsection{Pax6 knockdown in vitro studies}

In order to understand the role of Pax6 in alpha- and beta-cell function at the molecular level, Gosmain et al. (2010, 2012a, 2012b) used siRNA based approach to generate the Pax6 knockdown (KD) in primary rat alpha- and beta-cells. It was found that Pax6 can control the expression of several critical genes involved in the alpha- and beta-cell function. These included proglucagon, PC2, MafB, c-Maf, NeuroD1/Beta2, GK, GIPR, and GPR40 in alpha-cells; and insulin 1, insulin 2, Pdx1, MafA, Glut2, PC1/3, GK, Nkx6.1, c-Maf, PC2, GLP-1R, and GIPR in beta-cells. Additionally, Pax6 knockdown in alpha-cells imapirs the processing of proglucagon and secretion of glucagon due to decreased expression of the factors involved in proglucagon processing and glucagon secretion (Katz et al., 2009; Gosmain et al., 2012b). Moreover, Liu et al. (2012) showed that Pax6 can bind to the proSAAS (Pcsk1n) gene promoter and directly downregulate its 
expression, thereby controlling the PC1/3 activity and proper insulin processing in betacells.

These in vitro studies demonstrate that Pax6 is essential for both alpha- and beta-cell function as it controls the expression of many different genes involved in the synthesis, processing, and secretion of glucagon and insulin.

\subsubsection{Pax6 in diabetes (human studies)}

Several PAX6 mutations have been reported in humans (Prosser and Heyningen, 1998; Dansault et al., 2007). Heterozygous PAX6 mutations are a cause of aniridia (an eye disease) and are frequently linked to glucose intolerance (Yasuda et al., 2002). However, the etiology of age-induced glucose intolerance in aniridia patients is not completely understood. Wen et al. (2009) showed that defective proinsulin processing is one of the factors involved in the impairment of glucose tolerance in aniridia patients. 


\subsection{Aims of the study}

As discussed above, the Pax6 KO mice as well as Pax6 ${ }^{\text {sey/sey }}$ mutant mice die shortly after birth (Hill et al., 1991; St-Onge et al., 1997; Heller et al., 2005). This makes it impossible to analyze the role of Pax6 in adult endocrine pancreas in vivo. Later, the generation of Pax6 floxed mice allowed the ablation of Pax6 from the pancreas alone (Ashery-Padan et al., 2004). However, even these pancreas-specific conditional Pax6 KO mice do not survive beyond early postnatal stage. Recently, a study has been published where Pax6 was knocked out from adult pancreas with the help of an inducible ubiquitous Cre line (Hart et al., 2013). The ubiquitous Cre, however, removes Pax6 from all of the endocrine cell types at once and, therefore, does not allow the detection of cell-typespecific effects.

Our study aims at analyzing the role of Pax6 in adult pancreas in a cell-type-specific manner. For this purpose, we carried out conditional Pax6 KO in alpha- and beta-cells separately. This was done by using the Cre/loxP system (Sauer and Henderson, 1989; Rajewsky et al., 1996). Furthermore, YFP (yellow fluorescent protein) reporter transgene (Srinivas et al., 2001) was included to specifically mark the KO cells and to trace them over long-period of time. Along with that we also decided to increase the Pax6 dosage by overexpressing it in the whole pancreas, in the beta-cells alone, and in the alpha-cells alone. As an opposite approach to the knockout, overexpression can also provide useful information about the function of a protein.

This study was particularly aimed:

1. To identify and compare the characteristics of Pax6 KO alpha- and beta-cells. This would help us to know if Pax6 ablation affects both cell types in a similar or differential way.

2. To study the ghrelin ${ }^{+}$cell population in both types of KO pancreata, especially focusing on their origin and later fate. Ghrelin upregulation has been linked to Pax6 ablation in pancreas (Heller et al., 2005; Kordowich et al., 2011) but the origin of ghrelin ${ }^{+}$cells has not been exactly defined. 
3. To investigate the possibility of regeneration in alpha- and beta-cell population in adult pancreas using Pax6 KO as a model of glucagon and insulin deficiency.

4. To analyze gross changes in the hormonal cell population following Pax6 overexpression. It would be interesting to know, how increased Pax6 dosage affects pancreas development. 


\section{Materials and Methods}

\subsection{Materials}

\subsubsection{Primary antibodies}

Table 2.1: List of primary antibodies used.

\begin{tabular}{|c|c|c|c|}
\hline Antibody & Manufacturer & Host species & Working dilution \\
\hline Insulin & Sigma & Mouse & $1: 1000$ \\
\hline Insulin & Dako & Guinea pig & 1:1000 \\
\hline Glucagon & Abcam & Mouse & 1:1000 \\
\hline Glucagon & Abcam & Rabbit & $1: 50$ \\
\hline Somatostatin & Dako & Rabbit & $1: 600$ \\
\hline PP & Millipore & Rabbit & 1:1000 \\
\hline Ghrelin & Santa Cruz & Goat & $1: 50$ \\
\hline Ghrelin & Kindly provided by (1) & Mouse & 1:1000 \\
\hline Neurotensin & Abcam & Rabbit & $1: 200$ \\
\hline Pax6 & Covance & Rabbit & $1: 300$ \\
\hline Pax6 & DSHB & Mouse & $1: 100$ \\
\hline Isl1 & Abcam & Rabbit & $1: 500$ \\
\hline Rfx6 & Millipore & Rabbit & 1:2000 \\
\hline Pdx1 & Kindly provided by (2) & Rabbit & $1: 2000$ \\
\hline Nkx6.1 & DSHB & Mouse & $1: 50$ \\
\hline MafA & Bethyl Labs & Rabbit & $1: 500$ \\
\hline MafB & Bethyl Labs & Rabbit & 1:1000 \\
\hline Arx & Millipore & Rabbit & $1: 200$ \\
\hline Glut2 & Millipore & Rabbit & 1:1000 \\
\hline GLP-1 receptor & Kindly provided by (3) & Rabbit & $1: 4000$ \\
\hline C-peptide & Cell Signaling & Rabbit & $1: 100$ \\
\hline IAPP & Phoenix Pharma & Rabbit & $1: 500$ \\
\hline PC1/3 & Millipore & Rabbit & $1: 500$ \\
\hline PC2 & Millipore & Rabbit & $1: 200$ \\
\hline
\end{tabular}




\begin{tabular}{llll} 
7B2 & Abcam & Rabbit & $1: 5000$ \\
7B2 & Kindly provided by (4) & Rabbit & $1: 200$ \\
ProSAAS & Kindly provided by (4) & Rabbit & $1: 50$ \\
Ki67 & Dako & Rat & $1: 100$ \\
BrdU & Roche & Mouse & $1: 50$ \\
GFP & Abcam & Chicken & $1: 1000$ \\
$\beta$-galactosidase & Aves Labs & Chicken & $1: 5000$ \\
\hline
\end{tabular}

1. Dr. C. Tomasetto (Université Louis Pasteur, Strasbourg, France)

2. Dr. C. Wright (Vanderbilt University, Nashville, Tennessee)

3. Dr. J.F. Habener (Harvard Medical School, MA)

4. Dr. I. Lindberg University of Maryland-Baltimore, Maryland

DSHB=Developmental Studies Hybridoma Bank

\subsubsection{Secondary antibodies}

Table 2.2: List of secondary antibodies used.

\begin{tabular}{lllll}
\hline Conjugate & Host species & Reactivity & Working dilution & Manufacturer \\
\hline Alexa 488 & Goat & Rabbit & $1: 1000$ & Invitrogen \\
Alexa 594 & Goat & Rabbit & $1: 1000$ & Invitrogen \\
Alexa 594 & Donkey & Rabbit & $1: 1000$ & Invitrogen \\
Alexa 488 & Goat & Mouse & $1: 1000$ & Invitrogen \\
Alexa 594 & Goat & Mouse & $1: 1000$ & Invitrogen \\
Alexa 594 & Donkey & Mouse & $1: 1000$ & Invitrogen \\
Cy5 & Sheep & Mouse & $1: 300$ & Jackson Immuno- \\
Alexa 488 & Goat & Guinea Pig & $1: 1000$ & Research Labs, Inc \\
Alexa 594 & Goat & Guinea Pig & $1: 1000$ & Invitrogen \\
Alexa 594 & Goat & Rat & $1: 1000$ & Invitrogen \\
Alexa 488 & Chicken & Goat & $1: 1000$ & Invitrogen \\
Alexa 594 & Chicken & Goat & $1: 1000$ & Invitrogen \\
Alexa 488 & Goat & Chicken & $1: 1000$ & Invitrogen \\
Alexa 594 & Goat & Chicken & $1: 1000$ & Invitrogen \\
\hline
\end{tabular}




\subsubsection{Quantitative RT-PCR primers}

Table 2.3: List of qRT-PCR (quantitative reverse transcription - polymerase chain reaction) primers.

\begin{tabular}{ll}
\hline Gene & Primer \\
\hline Insulin & QuantiTect Primer Assay QT00258083 \\
Ghrelin & QuantiTect Primer Assay QT00137536 \\
Pax6 & QuantiTect Primer Assay QT01052786 \\
MafA & QuantiTect Primer Assay QT01037638 \\
Nkx6.1 & QuantiTect Primer Assay QT00143318 \\
Pdx1 & QuantiTect Primer Assay QT00102235 \\
Nkx2.2 & QuantiTect Primer Assay QT00495502 \\
Gusb & QuantiTect Primer Assay QT00176715 \\
\hline
\end{tabular}

\subsubsection{PCR primers for chromatin immunoprecipitation (ChIP)}

Table 2.4: List of PCR primers used in ChIP.

\begin{tabular}{|c|c|c|}
\hline Primer & Sequence & $\begin{array}{l}\text { Promoter-region } \\
\text { amplified }\end{array}$ \\
\hline Glut2-ChIP-F & CCTAAGACACAGAAAAGTCACAGGG & \multirow{2}{*}{$\begin{array}{l}-415 \text { to }-547 \text { (contains } \\
\text { proposed Pax6 } \\
\text { binding site) }\end{array}$} \\
\hline Glut2-ChIP-R & GTGGCCACAGAGTGTGGCAGCATCG & \\
\hline Ghrelin-ChIP-F & GGAGAAGCCGGTGAGCAGGCACCAC & \multirow{2}{*}{$\begin{array}{l}-335 \text { to }-476 \text { (contains } \\
\text { proposed Pax6 } \\
\text { binding site) }\end{array}$} \\
\hline Ghrelin-ChIP-R & CTGAATAATTTAGACCCCGGTGAGC & \\
\hline MafA-ChIP-F & CACCCCAGCGAGGGCTGATTTAATT & \multirow{2}{*}{$\begin{array}{l}-7750 \text { to }-8120 \\
\text { (Raum et al., 2010) }\end{array}$} \\
\hline MafA-ChIP-R & AGCAAGCACTTCAGTGTGCTCAGTG & \\
\hline
\end{tabular}




\subsubsection{PCR primers used in cloning}

Table 2.5: List of PCR primers used in cloning.

\begin{tabular}{|l|l|l|}
\hline No. & Primer & Sequence \\
\hline 1 & Pax6-SalI-forward & $\begin{array}{l}\text { TTTTTTTGTCGACATGCAGAACAGTCACAGCGGAGT } \\
\text { GAATCAGCTTGG }\end{array}$ \\
\hline 2 & Pax6-SalI-reverse & $\begin{array}{l}\text { TTTTTTTGTCGACTTACTGTAATCGAGGCCAGTACT } \\
\text { GAGACATGTCAG }\end{array}$ \\
\hline 3 & Pax6-seq-1 & CAGTATAAACGGGAGTGCCCTTC \\
\hline 4 & Pax6-seq-2 & CTTCTCTGGTTCCTCAGTTTCTC \\
\hline
\end{tabular}

\subsubsection{PCR primers used for genotyping}

Table 2.6: List of PCR primers used for genotyping.

\begin{tabular}{|l|l|l|}
\hline No. & Primer & Sequence \\
\hline 1 & Flp6-primer-83 & GCGGTTGAGTAGCTCAATTCTA \\
\hline 2 & Flp6-primer-84 & AGTGGCTTGGACTCCTCAAGA \\
\hline 3 & Flp6-primer-del & CGTGTGCCCCAGCTTCCGGT \\
\hline 4 & AM-89-Cre & ATG CTT CTG TCC GTT TGC CG \\
\hline 5 & AM-90-Cre & CCT GTT TTG CAC GTT CAC CG \\
\hline 6 & GFP-Forward & ACCCTGAAGTTCATCTGCACCA \\
\hline 7 & GFP-Reverse & TGGGTGCTCAGGTAGTGGTTGT \\
\hline
\end{tabular}

\subsubsection{Mouse lines used}

Table 2.7: List of mouse lines used.

\begin{tabular}{ll}
\hline Mouse line & Reference \\
\hline Pax6 floxed & Ashery-Padan et al., 2000
\end{tabular}




$\begin{array}{ll}\text { RIP-CreER } & \text { Dor et al., } 2004 \\ \text { Glucagon-Cre } & \text { Herrera, 2000 } \\ \text { Insulin-Cre } & \text { Herrera, 2000 } \\ \text { Pdx1-Cre } & \text { Gannon et al., } 2000 \\ \text { R26-YFP reporter } & \text { Srinivas et al., } 2001\end{array}$

\subsubsection{Solutions and Media}

Table 2.8: Phosphate buffered saline (PBS).

\begin{tabular}{ll}
\hline Component & Amount (g/L) \\
\hline $\mathrm{NaCl}$ & 8.0 \\
$\mathrm{KCl}$ & 0.2 \\
$\mathrm{Na}_{2} \mathrm{HPO}_{4} \cdot 2 \mathrm{H}_{2} \mathrm{O}$ & 1.35 \\
$\mathrm{NaH}_{2} \mathrm{PO}_{4} \cdot 2 \mathrm{H}_{2} \mathrm{O}$ & 0.1 \\
$\mathrm{KH}_{2} \mathrm{PO}_{4}$ & 0.2 \\
\hline
\end{tabular}

Dissolve in Milli-Q water and bring the final volume to 1 litre.

Table 2.9: Luria-Bertani medium (LB medium).

\begin{tabular}{ll}
\hline Component & Amount (g/L) \\
\hline Tryptone & 10 \\
Yeast extract & 5 \\
$\mathrm{NaCl}$ & 10 \\
\hline
\end{tabular}

Dissolve in $900 \mathrm{~mL}$ Milli-Q water, adjust the $\mathrm{pH}$ to 7.0 and bring the final volume to 1 liter. The medium was autoclaved before use.

\section{LB agar plates}

For LB agar plates, add 15 g/L agar to LB medium before autoclaving. After autoclaving, let the medium cool down to $\approx 50^{\circ} \mathrm{C}$, add the antibiotic (e.g., ampicillin), and pour into sterile plates. After the medium has solidified, invert the plates and store at $4{ }^{\circ} \mathrm{C}$ in dark. 


\subsection{Methods}

\subsubsection{Animal treatments}

\subsubsection{Tamoxifen treatment}

Tamoxifen was prepared in corn oil (at $20 \mathrm{mg} / \mathrm{ml}$ ). For 3 week old mice, $1 \mathrm{mg}$ tamoxifen was administered intraperitoneally for 3 days every other day. For 1.5 month and 2 month old mice, 2 mg tamoxifen was administered intraperitoneally for 4 days every other day.

\subsubsection{Bromodeoxyuridine (BrdU) treatment}

BrdU was given in drinking water (at $0.8 \mathrm{mg} / \mathrm{mL}$ ) to the mice for 3 days. Water bottles were wrapped in aluminium foil to protect from light-mediated degradation.

\subsubsection{Blood and urine glucose measurement}

For glucose measurements One Touch Glucose meter (Johnson \& Johnson) was used. A drop of blood from tail vein or a drop of urine was directly taken on to the test strip.

\subsubsection{Histological procedures}

\subsubsection{Pancreas preparation, cryo-embedding, and sectioning}

Mice were killed by decapitation (at $\mathrm{P} 0$ ) and by cervical dislocation or $\mathrm{CO}_{2}$ inhalation (at age of 3 weeks or more). Pancreata were removed and immediately placed in ice-cold PBS (Table 2.8). It was followed by washing in PBS (3x10minutes) at $4^{\circ} \mathrm{C}$. After washing the pancreata were fixed in 4\% paraformaldehyde (dissolved in PBS; pH 7.4) for 1 hour at $4^{\circ} \mathrm{C}$. Fixed pancreata were then washed in PBS (4x20minutes) and placed in $25 \%$ sucrose (dissolved in PBS) for overnight at $4^{\circ} \mathrm{C}$. After this pancreata were washed two times in Jung Tissue Freezing Medium ${ }^{\mathrm{TM}}$ (Leica Microsystems) (2x2hours), and embedded in the same medium on dry ice using cryomolds. Frozen embedded pancreata were stored at $-20^{\circ} \mathrm{C}$ or $-80^{\circ} \mathrm{C}$.

From the frozen pancreata, $8 \mu \mathrm{m}$ sections were cut on a cryostat and mounted on SuperFrost ${ }^{\circledR}$ Plus slides (Thermo Scientific). Slides were kept at $30^{\circ} \mathrm{C}$ for half an hour, to allow the adhesion of sections, before being stored at $-20^{\circ} \mathrm{C}$ or $-80^{\circ} \mathrm{C}$. 


\subsubsection{Immunofluorescence staining of cryosections}

Pancreatic cryosections on each slide were encircled with ImmEdge ${ }^{\mathrm{TM}}$ pen. Slides were then washed in PBS (3x5minutes). Next, the sections were blocked with 10\% fetal calf serum (in PBS containing $0.1 \%$ triton $\mathrm{x}-100$ ) for 1 hour at room temperature. It was followed by incubation of the sections with primary antibodies (diluted in the same blocking solution) at $4^{\circ} \mathrm{C}$ for overnight. Next day, the slides were washed in PBS (3x5minutes). After washing, the sections were incubated with the appropriate secondary antibodies (diluted in the same blocking solution) for 1 hour at room temperature. Afterwards, the slides were washed again in PBS (3x5minutes) and mounted with Vectashield ${ }^{\circledR}$ mounting medium containing DAPI (4',6-diamidino-2-phenylindole) (Vector Labs).

\subsubsection{BrdU staining}

Slides with cryosections were washed in PBS for 15 minutes and incubated in $2 \mathrm{~N} \mathrm{HCl}$ for 30 minutes at $37^{\circ} \mathrm{C}$. This was followed by washing of slides in PBS (2x30minutes). Next, the cryosections on each slide were encircled with ImmEdge ${ }^{\mathrm{TM}}$ pen and blocked with $10 \%$ fetal calf serum (in PBS containing $0.1 \%$ triton $\mathrm{x}-100$ ) for 1 hour at room temperature. It was followed by incubation of the sections with primary antibodies (diluted in the same blocking solution) at $4^{\circ} \mathrm{C}$ for overnight. Next day, the slides were washed in PBS (2x15minutes). After washing, the sections were incubated with the appropriate secondary antibodies (diluted in the same blocking solution) for 1 hour at room temperature. Afterwards, the slides were washed again in PBS (2x30minutes) and mounted with Vectashield ${ }^{\circledR}$ mounting medium containing DAPI (Vector Labs).

\subsubsection{TUNEL staining}

TUNEL (terminal deoxynucleotidyl transferase dUTP nick end labeling) assay detects apoptotic cells by labeling fragmented DNA resulting from apoptosis. For TUNEL staining, Apop Tag ${ }^{\circledR}$ Red In Situ Apoptosis Detection Kit (Millipore) was used and the procedure was performed according to the manufacturer's instructions.

\subsubsection{X-Gal staining}

For X-Gal (5-bromo-4-chloro-indolyl- $\beta$-D-galactopyranoside) staining, pancreata were prepared and embedded in the same way as mentioned before (section 2.2.2.1). However, 
the fixative used was different. These pancreata were fixed in fixative A (1\% formaldehyde, $0.2 \%$ glutaraldehyde, $0.02 \%$ NP-40, in PBS) for 30 minutes and then in fixative B (1\% formaldehyde, 0.2\% glutaraldehyde, $0.2 \%$ NP-40, $0.1 \%$ sodium deoxycholate, in PBS) for 1 hour at $4^{\circ} \mathrm{C}$. From the embedded pancreata, $8 \mu \mathrm{m}$ sections were cut. The cryosections were fixed again in ice-cold $0.2 \%$ glutaraldehyde (in PBS) for 10 minutes. Next, the sections were washed in X-Gal staining buffer $\left(2 \mathrm{mM} \mathrm{MgCl}_{2}\right.$, 0.02\% NP-40, 0.01\% sodium deoxycholate, in PBS) at room temperature (3x5minutes). Then the sections were overlayed with X-Gal staining solution $(1 \mathrm{mg} / \mathrm{mL}$ X-Gal, $5 \mathrm{mM}$ $\mathrm{K}_{3} \mathrm{Fe}(\mathrm{CN})_{6}, 5 \mathrm{mM} \mathrm{K}_{4} \mathrm{Fe}(\mathrm{CN})_{6}, 2 \mathrm{mM} \mathrm{MgCl}_{2}, \quad 0.02 \% \quad \mathrm{NP}-40,0.01 \%$ sodium deoxycholate, in PBS) and incubated at $30^{\circ} \mathrm{C}$ for $24-72$ hours in dark. For staining longer than 24 hours, the staining solution was changed after every 24 hours. After completion of staining, the slides were washed in PBS (2x5minutes) and the sections were mounted with Vectashield ${ }^{\circledR}$ mounting medium containing DAPI (Vector Labs). Images of X-Gal staining were acquired on Olympus BX 60 fluorescent microscope at 20x magnification.

\subsubsection{Imaging}

For quantification of islet cell numbers, images were acquired on Olympus BX 60 fluorescent microscope at 20x magnification. All other images were acquired using a 40x oil-immersion objective on Leica TCS SP5 laser scanning confocal microscope.

\subsubsection{Quantification of islet cell numbers}

The whole pancreata were cut into serial sections and every $20^{\text {th }}$ section was stained with antibodies against different hormone/hormone combinations. Cells were counted from all the islets on every section and an average no./islet was calculated. Quantification data is presented as mean \pm Standard error of mean (SEM). To measure statistical significance, unpaired student's two-tailed t-test was applied and $\mathrm{p}<0.05$ was considered as significant.

\subsubsection{Cell culture}

Mouse insulinoma (Min6) cells were maintained in Dulbecco’s modified eagle medium (Gibco®-containing $25 \mathrm{mM}$ glucose and $3.97 \mathrm{mM}$ L-Glutamine) supplemented with $10 \%$ fetal calf serum, 1X penicillin/streptomycin (Gibco®-100U/mL penicillin and $100 \mu \mathrm{g} / \mathrm{ml}$ streptomycin) and beta-mercaptoethanol $(5 \mu \mathrm{L} / \mathrm{L})$. The cells were grown at $37^{\circ} \mathrm{C}$ in the presence of $5 \% \mathrm{CO}_{2}$. 


\subsubsection{Immunofluorescent staining of cultured cells}

For immunofluorescent staining, Min6 cells were cultured in four-chamber slides (Thermo Scientific). Medium was removed and cells were washed once with PBS. Then, the cells were fixed with 3.6\% formaldehyde (in PBS) for 20 minutes at room temperature. It was followed by washing with PBS (2x5minutes). Next, the cells were permeabilized with $0.5 \%$ triton $\mathrm{x}-100$ (in PBS) for 10 minutes and washed again with PBS (1x5minutes). After washing, the cells were blocked with $10 \%$ fetal calf serum (in PBS containing $0.1 \%$ triton $\mathrm{x}-100$ ) for 1 hour at room temperature. Then the cells were incubated with primary antibodies (diluted in the same blocking solution) at $4^{\circ} \mathrm{C}$ for overnight. Next day, the cells were washed with PBS (3x5minutes). After washing, the cells were incubated with the appropriate secondary antibodies (diluted in the same blocking solution) for 1 hour at room temperature. Afterwards, the cells were washed again with PBS (3x5minutes). The slide chamber was then removed and cells were mounted with Vectashield ${ }^{\circledR}$ mounting medium containing DAPI (Vector Labs).

\subsubsection{Molecular Biology}

\subsubsection{Chromatin immunoprecipitation assay}

For chromatin immunoprecipitation, EZ-ChIP ${ }^{\mathrm{TM}}$ kit (Millipore) was used and the procedure was performed according to manufacturer's instructions. Briefly, Min6 cells were cultured in $10 \mathrm{~cm}$ dish to $90 \%$ confluency. Formaldehyde was directly added to culture medium (1\% final concentration) to fix the cells at room temperature for 10 minutes. Fixation was stopped by adding glycine to the medium (125 mM final concentration) and incubating for 5 minutes at room temperature. All the next steps were performed on ice. Cells were washed twice in PBS and collected in $2 \mathrm{~mL}$ PBS containing protease inhibitor cocktail. After centrifugation the pellet was resuspended in $1 \mathrm{~mL}$ lysis buffer. This cell suspension was sonicated to shear the crosslinked DNA to a fragment length of $\approx 200-1000$ base pairs. The sonicated suspension was centrifuged to remove the insoluble material. After centrifugation, $100 \mu \mathrm{L}$ of the supernatant was diluted to $1 \mathrm{~mL}$ with dilution buffer containing protease inhibitor cocktail. The diluted chromatin was precleared by incubating with Protein G Agarose beads for 1 hour at $4^{\circ} \mathrm{C}$ on a rotating platform. At this step, $1 \%$ of the chromatin sample was set aside as input. The rest of precleared chromatin was incubated, with $10 \mu \mathrm{g}$ of rabbit anti-Pax 6 antibody or $10 \mu \mathrm{g}$ of 
normal rabbit IgG (Millipore), for overnight at $4^{\circ} \mathrm{C}$ on a rotating platform. Next day the antibody-protein-DNA complexes were collected by incubating with Protein G Agarose beads for 1 hour at $4^{\circ} \mathrm{C}$ with rotation. The complexes were then washed through a series of wash buffers and eluted with elution buffer. Finally, the protein-DNA crosslinks were removed and the DNA was purified. This purified DNA was then used for PCR.

To check the binding of Pax6 with various promoter regions, PCR was performed with the primer sets mentioned in Table 2.4. Reaction was carried out in eppendorf Mastercycler®. PCR reaction composition and the program used were as follows:

\begin{tabular}{|l|l|}
\hline Reaction component & Volume $(\boldsymbol{\mu L})$ \\
\hline DNA & 2.0 \\
\hline $\mathrm{H}_{2} \mathrm{O}$ & 12.6 \\
\hline $5 \mathrm{X}$ PCR buffer & 4 \\
\hline 20mM dNTP & 0.2 \\
\hline Primer (forward) & 0.4 \\
\hline Primer (reverse) & 0.4 \\
\hline $\begin{array}{l}\text { Go Taq } \\
\text { (5U/ DNA) (Promega) }\end{array}$ & 0.4 \\
\hline
\end{tabular}

PCR program used was as follows:

Initial denaturation $94^{\circ} \mathrm{C}-3$ minutes, 35 cycles (denaturation $94^{\circ} \mathrm{C}-20$ seconds, annealing $59^{\circ} \mathrm{C}-30$ seconds, extension $72^{\circ} \mathrm{C}-30$ seconds), final extension $72^{\circ} \mathrm{C}-5$ minutes.

\subsubsection{Tail DNA isolation}

Genomic DNA was isolated from tail-tip biopsy samples. For tissue lysis, $0.5 \mathrm{~mL}$ tail lysis buffer (100 mM Tris-pH 8.0, 5 mM EDTA, 0.2\% SDS, $200 \mathrm{mM} \mathrm{NaCl}$, supplemented with $250 \mu \mathrm{g} / \mathrm{ml}$ Proteinase K) was added to each cut-tail taken in a $1.5 \mathrm{~mL}$ eppendorf tube. Samples were then incubated at $55^{\circ} \mathrm{C}$ for overnight on a shaker. Next day, the samples were centrifuged at 13,000 rpm for 6 minutes. The supernatant from each sample was transferred to a new $1.5 \mathrm{~mL}$ eppendorf tube. Next, to precipitate the DNA, $0.5 \mathrm{~mL}$ isopropanol was added to each sample and inverted several times to mix well. This was followed by centrifugation at 13,000 rpm for 6 minutes to collect the DNA 
pellets. Supernatant was discarded and $0.5 \mathrm{~mL} \mathrm{70 \%} \mathrm{ethanol} \mathrm{was} \mathrm{added} \mathrm{to} \mathrm{each} \mathrm{DNA}$ pellet for washing. Next, the samples were centrifuged again at 13,000 rpm for 6 minutes. Supernatant was discarded and pellets were allowed to air-dry. Dried pellets were dissolved in autoclaved Milli-Q water $(60 \mu \mathrm{L}$ per pellet $)$ and stored at $4{ }^{\circ} \mathrm{C}$.

\subsubsection{Genotyping of mice}

Genotyping of mouse lines was done by PCR using the DNA isolated from tails. Pax6wild-type, floxed allele, and deleted allele was detected by using primer number 1 , 2, and 3 (Table 2.6 - as used in Piñon et al., 2008). Cre transgene and YFP reporter transgene was detected by using primer number $4 / 5$ and 6/7, respectively (Table 2.6). Reactions were carried out in eppendorf Mastercycler ${ }^{\circledR}$. The same PCR reaction composition was used for all genotyping PCRs but different PCR programs were used as follows:

\begin{tabular}{|l|l|}
\hline Reaction component & Volume $(\boldsymbol{\mu} \mathbf{L})$ in total of $30 \boldsymbol{\mu L}$ \\
\hline $\mathrm{H}_{2} \mathrm{O}$ & 22.3 \\
\hline $5 \mathrm{X}$ buffer & 6.0 \\
\hline dNTPs $(20 \mathrm{mM})$ & 0.38 \\
\hline Primers $(10 \mu \mathrm{M})$ & 0.16 (each) \\
\hline $\begin{array}{l}\text { Go Taq }{ }^{\circledR} \text { DNAPolymerase } \\
(5 \mathrm{U} / \mu \mathrm{L})(\text { Promega) }\end{array}$ & 0.16 \\
\hline Template DNA & 1 \\
\hline
\end{tabular}

Pax6-flox PCR program:

Initial denaturation $95^{\circ} \mathrm{C}-4$ minutes, 30 cycles (denaturation $95^{\circ} \mathrm{C}-50$ seconds, annealing $58^{\circ} \mathrm{C}-50$ seconds, extension $68^{\circ} \mathrm{C}-50$ seconds), final extension $72^{\circ} \mathrm{C}-10$ minutes.

Cre PCR program:

Initial denaturation $95^{\circ} \mathrm{C}-4$ minutes, 30 cycles (denaturation $95^{\circ} \mathrm{C}-30$ seconds, annealing $57^{\circ} \mathrm{C}-30$ seconds, extension $65^{\circ} \mathrm{C}-30$ seconds), final extension $72^{\circ} \mathrm{C}-10$ minutes. 


\section{GFP PCR program:}

Initial denaturation $95^{\circ} \mathrm{C}-4$ minutes, 35 cycles (denaturation $94^{\circ} \mathrm{C}-30$ seconds, annealing $62^{\circ} \mathrm{C}-30$ seconds, extension $72^{\circ} \mathrm{C}-30$ seconds), final extension $72^{\circ} \mathrm{C}-10$ minutes.

\subsubsection{Total RNA isolation, cDNA synthesis, and qRT PCR}

For RNA isolation, pancreata were placed in RNAlater (Qiagen) immediately after removal. Pancreata were kept in RNAlater for overnight at $4^{\circ} \mathrm{C}$. Next day they were either used for RNA isolation or transferred to $-20^{\circ} \mathrm{C}$ for long-term storage. For total RNA isolation, pancreatic tissue was first disrupted using Tissuelyser (Qiagen). Total RNA was then extracted from the tissue lysate by using RNeasy Plus Mini Kit (Qiagen) according to the manufacturer's instructions. RNA quality was checked by spectrometry on NanoDrop (Peqlab) and by agarose gel electrophoresis. For cDNA synthesis, SuperScript II Reverse Transcriptase Kit (Invitrogen) was used according to the manufacturer's instructions. Finally, this cDNA was used for quantitative real-time PCR using primers mentioned in Table 2.3. Beta-glucuronidase (Gusb) was used as a house keeping control. PCR was carried out in Mastercycler ${ }^{\circledR}$ realplex ${ }^{2}$ (Eppendorf) using SYBR Green Master Mix (Qiagen) according to manufacturer’s instructions.

\subsubsection{Generation of calcium competent cells}

A single colony of E. coli (DH5 $\alpha$ ) was inoculated into $5 \mathrm{~mL} \mathrm{LB}$ medium (Table 2.9) and incubated for overnight at $37^{\circ} \mathrm{C}$ on shaker. Next day this $5 \mathrm{~mL}$ pre-culture was added to $50 \mathrm{~mL} \mathrm{LB}$ medium and incubated further at $37^{\circ} \mathrm{C}$ on shaker until an $\mathrm{OD}_{600}$ of 0.5 was achieved. Then the bacteria were pelleted by centrifugation at $4^{\circ} \mathrm{C}$ (3000g, 8 minutes). The pellet was resuspended in $25 \mathrm{~mL}$ ice-cold $\mathrm{CaCl}_{2}(50 \mathrm{mM})$ and incubated on ice for 30 minutes. It was followed by centrifugation again at $4^{\circ} \mathrm{C}$ (3000g, 4 minutes). Supernatant was discarded and the pellet was resuspended in $2.5 \mathrm{~mL}$ ice-cold $\mathrm{CaCl}_{2}$ (50 $\mathrm{mM}$ ). For storage, $1 \mathrm{~mL}$ of $50 \%$ glycerol was added and the competent cells were stored in $200 \mu \mathrm{L}$ aliquots at $-80^{\circ} \mathrm{C}$.

\subsubsection{Transformation of competent cells}

Competent cells stored at $-80^{\circ} \mathrm{C}$ were thawed on ice for 10 minutes. Purified plasmid DNA (50 ng) or ligation mixture (10 $\mu \mathrm{L})$ was added to competent cells and incubated on 
ice for 30 minutes. Next, the competent cells were given a heat shock at $42^{\circ} \mathrm{C}$ for 90 seconds. After heat shock cells were put back on ice for 2 minutes. Then $800 \mu \mathrm{L} \mathrm{LB}$ medium was added to transformed competent cells and the cells were incubated at $37^{\circ} \mathrm{C}$ for 1 hour on shaker. Finally, the transformed cells were plated on LB agar plates supplemented with appropriate selection antibiotic. The plates were incubated at $37^{\circ} \mathrm{C}$ for overnight. Next day the plates were observed for the appearance of resistant clones.

\subsubsection{Plasmid DNA isolation}

Plasmid DNA from bacteria was isolated by using the Qiagen Mini prep and High Speed Midi prep kits. DNA concentration was measured by spectrometry using NanoDrop (Peqlab).

\subsubsection{Agarose gel electrophoresis and gel extraction of DNA fragments}

Depending on the size of DNA fragments to be analyzed, a 0.8 - $2 \%$ agarose gel was prepared. Agarose (Roti®garose-Roth) was dissolved by melting in 0.5X TBE buffer (44.5 mMTris-base, $44.5 \mathrm{mM}$ boric acid, $1 \mathrm{mM}$ EDTA-pH 8.0). To visulaize the DNA after electrophoresis, ethidium bromide was directly added to the gel at a final concentration of $0.2 \mu \mathrm{g} / \mathrm{mL}$. DNA was mixed with $6 \mathrm{X}$ DNA loading dye $(30 \% \mathrm{v} / \mathrm{v}$ glycerol, $0.25 \%$ bromophenol blue, $0.25 \%$ xylene cyanol FF) before loading. GeneRuler ${ }^{\mathrm{TM}} 1 \mathrm{~kb}$ and 100 bp DNA ladders were used for estimation of band sizes. TBE (0.5X) was used as a running buffer and electrophoresis was performed at 4-5 volts per $\mathrm{cm}$. Afterwards the DNA fragments were visualized under a UV transilluminator. For gel extraction, the DNA band of the required size was cut out of the gel and purified by using Gene Clean ${ }^{\circledR}$ Spin Kit (MPIbio), according to the manufacturer’s instructions.

\subsubsection{Restriction digestion and dephosphorylation}

For restriction digestion, 1 to $5 \mu \mathrm{g}$ of plasmid DNA or purified PCR product was incubated with 20 to 100 units of restriction enzyme (New England BioLabs) and appropriate buffer in the presence or absence of bovine serum albumin. The digestion was carried out at $37^{\circ} \mathrm{C}$ for 4 to 8 hours. For dephosphorylation of vector backbone antarctic phosphatase (New England BioLabs) and the associated buffer were directly added to the restriction digest mixture $\left(10 \mathrm{U} / \mu \mathrm{g}\right.$ of DNA) and incubated for 1 hour more at $37^{\circ} \mathrm{C}$. 


\subsubsection{Ligation}

Ligation was carried out using T4 DNA ligase at $16^{\circ} \mathrm{C}$ for overnight. Ligation mixture contained: $2 \mu \mathrm{L}$ vector DNA (50 ng/ $\mu \mathrm{L}), 6 \mu \mathrm{L}$ insert DNA $(50 \mathrm{ng} / \mu \mathrm{L}), 1 \mu \mathrm{L}$ ligation buffer (10X), $1 \mu \mathrm{L}$ T4 DNA ligase (400 U/ $\mu \mathrm{L})$. Next day the ligation mixture was directly used for transformation.

\subsubsection{Generation of Pax6 overexpression construct}

Pax6cDNA was amplified by PCR using forward and reverse primers containing SalI restriction site (Table 2.5). CMV-Pax6 plasmid (Walther and Gruss, 1991) was used as a template. Reaction was carried out in eppendorf Mastercycler ${ }^{\circledR}$. Composition of the reaction mixture and PCR program used was as follows:

\begin{tabular}{|l|l|}
\hline Reaction component & Volume $(\boldsymbol{\mu L})$ in total of $50 \boldsymbol{\mu L}$ \\
\hline $\mathrm{H}_{2} \mathrm{O}$ & 40.5 \\
\hline $10 \mathrm{X}$ buffer & 5 \\
\hline $\mathrm{dNTPs}(20 \mathrm{mM})$ & 0.5 \\
\hline Primer-forward $(10 \mu \mathrm{M})$ & 1 \\
\hline Primer-reverse $(10 \mu \mathrm{M})$ & 1 \\
\hline Template DNA $(100 \mathrm{ng} / \mu \mathrm{L})$ & 1 \\
\hline $\begin{array}{l}\text { Taq DNA Polymerase }(1 \mathrm{U} / \mu \mathrm{L}) \\
\text { (Roche) }\end{array}$ & 1 \\
\hline
\end{tabular}

PCR program used:

Initial denaturation $95^{\circ} \mathrm{C}-4$ minutes, 35 cycles (denaturation $95^{\circ} \mathrm{C}-40$ seconds, annealing $60^{\circ} \mathrm{C}-40$ seconds, extension $72^{\circ} \mathrm{C}-90$ seconds), final extension $72^{\circ} \mathrm{C}-20$ minutes.

PCR product was purified by gel extraction and digested with SalI restriction enzyme. The destination vector pJojo (Collombat et al., 2007) was digested with XhoI and dephosphorylated to prevent self-ligation. The digested PCR product and vector backbone were again purified by gel extraction. This was followed by the overnight ligation of PCR product and vector backbone. Next day the ligation mixture was transformed into competent E. coli $(\mathrm{DH} 5 \alpha)$ and the transformed cells were plated on LB-agar plates 
supplemented with $50 \mu \mathrm{g} / \mathrm{mL}$ ampicillin. The plates were incubated at $37^{\circ} \mathrm{C}$ for overnight. Next day 10 single colonies were picked and each was inoculated into $5 \mathrm{~mL}$ LB medium (supplemented with $50 \mu \mathrm{g} / \mathrm{mL}$ ampicillin) in a sterile tube. The tubes were incubated on shaker at $37^{\circ} \mathrm{C}$ for overnight. Next day the plasmid DNA was isolated. Finally, the correct orientation and sequence of the insert was confirmed by sequencing with the primers (3/4) mentioned in Table 2.5. For sequencing, Seqlab (Goettingen) sequencing service was used. The clone with the correct orientation and sequence was linearized by digesting with SalI restriction enzyme and purified by gel extraction. This purified linear construct was then used for pronuclear injection to generate the transgenic mouse lines. 


\section{Results}

\subsection{Analysis of beta-cell-specific Pax6 knockout pancreata}

\subsubsection{Inducible conditional knockout of $\operatorname{Pax} 6$ in the adult pancreatic beta-cells}

Pax6 classical as well as pancreas-specific conditional knockout mice die shortly after birth. Death of these mice results from an overt diabetic phenotype that points to the essential role of Pax6 in the maintenance of beta-cell function (Ashery-Padan et al., 2004). However, due to this early postnatal lethality it is impossible to analyze the role of Pax6 in adult beta-cells. For this reason, we decided to generate inducible Pax6 knockout (KO) mice where Pax6 is ablated upon tamoxifen induction in beta-cells alone without affecting other endocrine cell types. In order to generate the mice with the required genotype, Pax6 floxed mouse line (Pax6 $6^{f l / f l}$ ) (Ashery-Padan et al., 2000) was crossed with RIP-CreER mouse line (Dor et al., 2004). Secondarily, we decided to incorporate the YFP reporter that allows the identification and tracing of Cre-recombined cells (Figure 3.1). This was achieved by including an additional cross with R26-YFP reporter mouse line (Srinivas et al., 2001). Mice with the right genotype were injected with tamoxifen and the ablation of Pax6 from beta-cells was confirmed by double immunofluorescence staining (Figure 3.2a-d). The efficiency of Pax6 ablation from beta-cells was nearly 95\% as indicated by quantification of the Pax6 ${ }^{-}$insulin $^{+}$cells (Figure 3.2e). A similar labeling efficiency was obtained with the YFP reporter (Figure 3.2f). It was further confirmed by immunofluorescence staining that Pax6 is specifically deleted from the YFP labeled cells and the $\mathrm{YFP}^{-}$insulin ${ }^{+}$cells that either escape recombination and/or arise later as a result of regeneration are positive for Pax6 expression (Figure 3.2g-j). Thus, Cre is active in 95\% of beta-cells and the ablation of Pax6 and labeling with the YFP reporter are faithfully linked to each other.

\subsubsection{Development of diabetes in the beta-cell-specific Pax6 KO mice}

As Pax6 has been shown to be implicated in the control of beta-cell function, an increase in the level of blood glucose was expected (Ashery-Padan et al., 2004; Gosmain et al., 2012a). Therefore, we monitored the non-fasting blood glucose level in control and betacell specific Pax6 KO mice following the tamoxifen injection. As compared to the control mice, blood glucose level started to rise in the $\mathrm{KO}$ mice within a week after first injection 

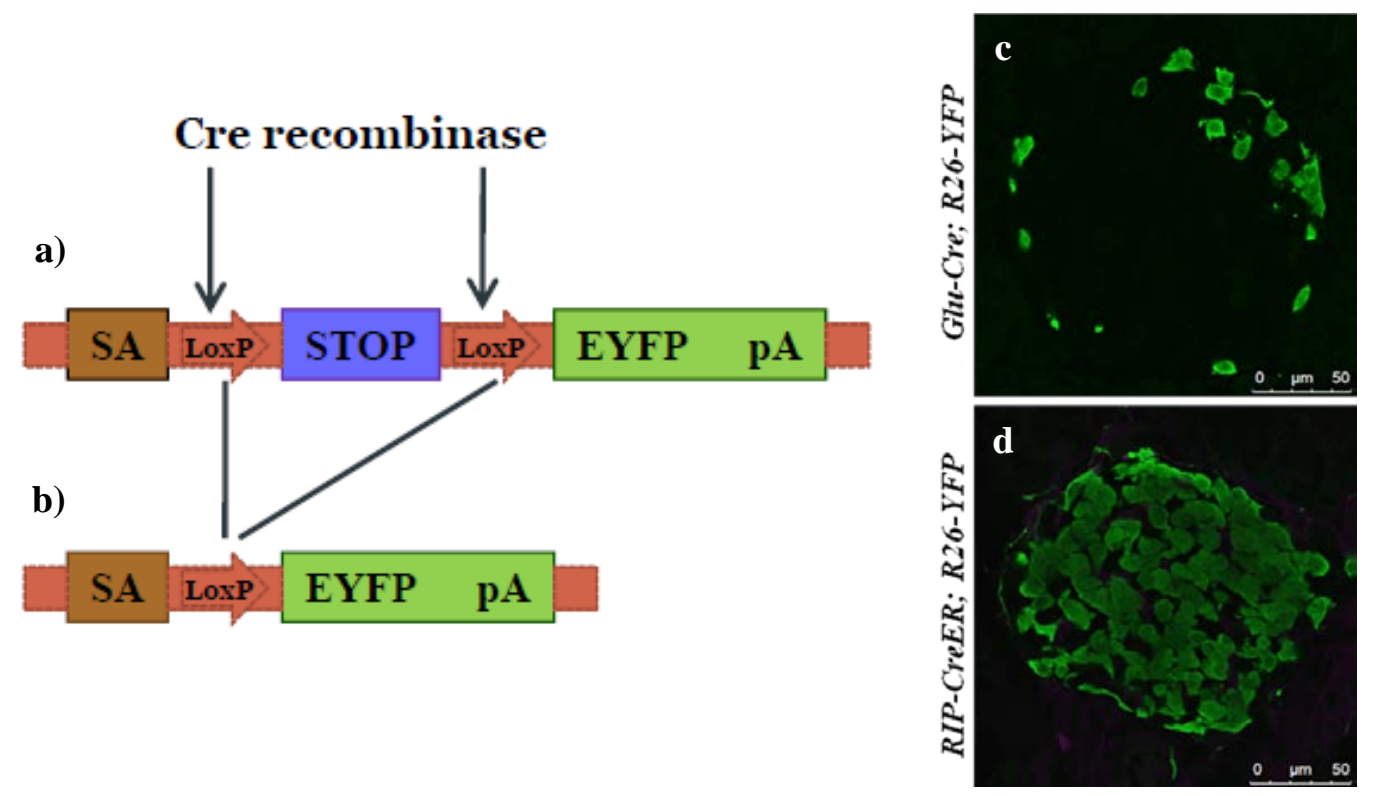

Figure 3.1: Lineage tracing with $R$ 26-YFP reporter mouse line. (a) The "stop cassette" flanked by loxP sites includes a trascriptional stop that does not allow the expression of YFP in the absence of Cre activity. (b) Following Cre-mediated recombination "stop" is removed allowing the permanent expression of YFP in Cre expressing cells. (c) YFP Labeling of alpha-cells expressing Cre under glucagon promoter. (d) YFP labeling of beta-cells expressing Cre under rat insulin II promoter (RIP). (R26=Rosa26; SA=splice acceptor) 

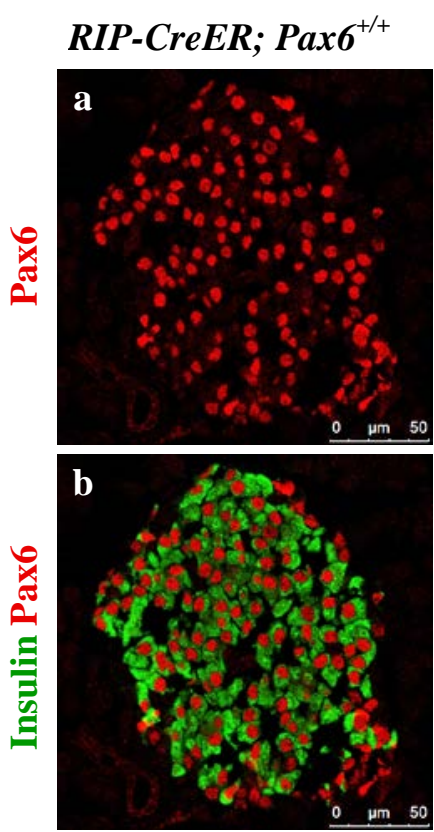

RIP-CreER; R26-YFP; Pax6 $^{+/+}$
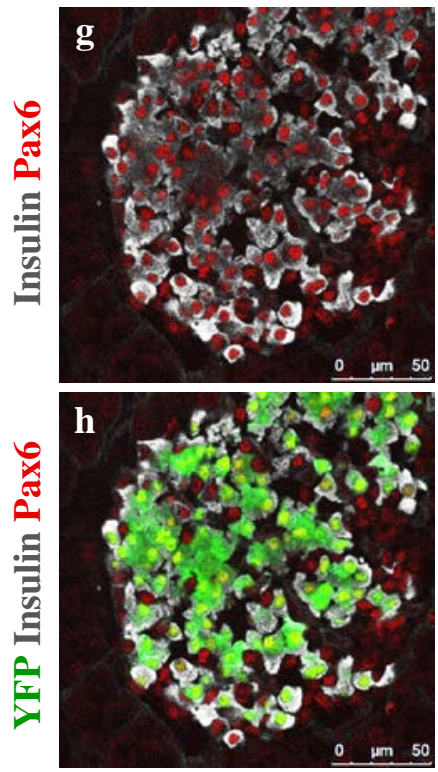

\section{RIP-CreER; Pax6 ${ }^{f l f l}$}
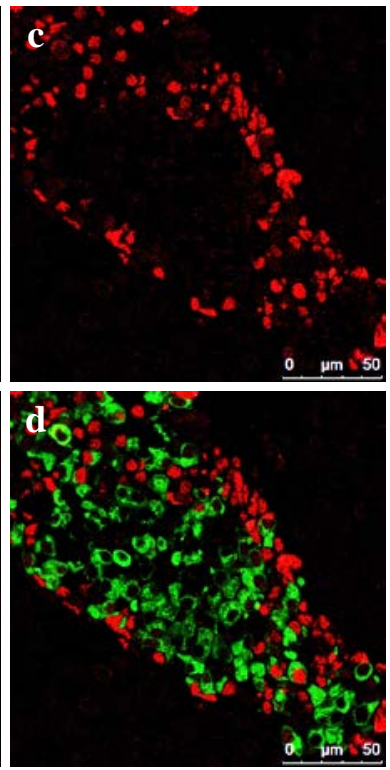

RIP-CreER; R26-YFP; Pax6 ${ }^{f l / l}$
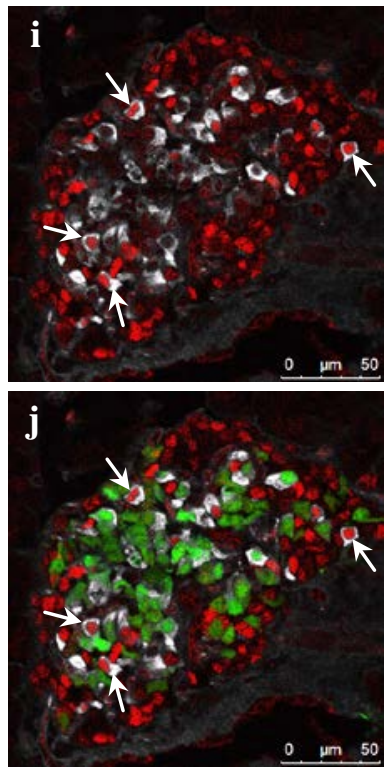

e)

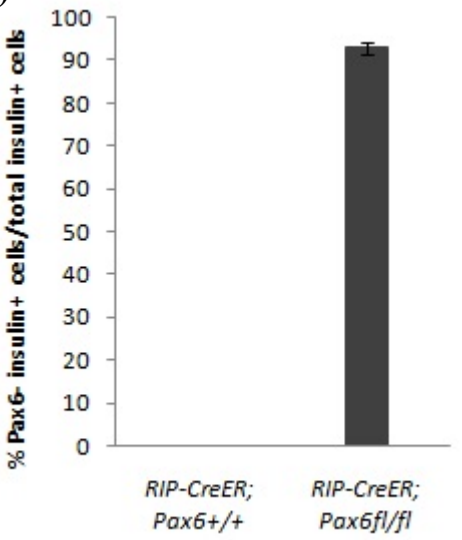

f)

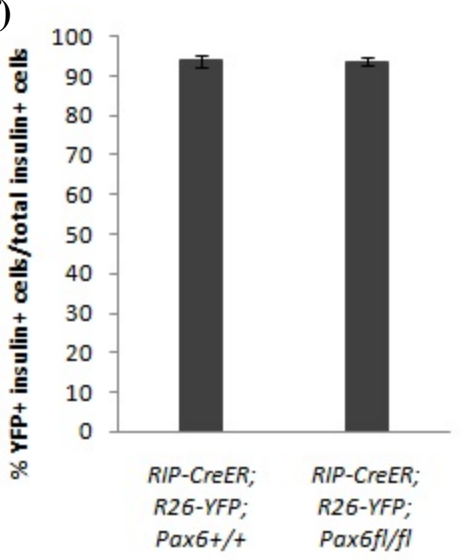

Figure 3.2: Beta-cell-specific ablation of Pax6. Double immunofluorescence staining of pancreatic cryosections from 2 month old mice at 4 days (a-d) or 4 weeks (g-j) after tamoxifen induction. Pax6 expression is lost from majority of the insulin ${ }^{+}$cells (c, d). Ablation of Pax6 is specific to YFP labeled cells (i,j) as $\mathrm{YFP}^{-}$insulin ${ }^{+}$cells continue to express Pax6 (arrows i,j). Quantification of Pax6 ${ }^{-}$insulin $^{+}$(e) and $\mathrm{YFP}^{+}$insulin ${ }^{+}$(f) cells in the pancreata of 2 month old mice at 4 days after tamoxifen induction $(n=3)$. Cremediated recombination/Pax6 ablation takes place in nearly 95\% of beta-cells. Error bars represent SEM. 
of tamoxifen. At four weeks after injection the blood glucose level in the KO mice was $\approx 450 \mathrm{mg} / \mathrm{dL}$ on average compared to $\approx 100 \mathrm{mg} / \mathrm{dL}$ average level in the control mice. At this time point a high glucose level was also detected in the urine of KO mice. Hence, the ablation of Pax6 from beta-cells leads to an overt diabetic phenotype and supports the essential role of Pax6 in the maintenance of adult beta-cell function.

\subsubsection{Expression of pancreatic endocrine hormones in the beta-cell-specific Pax6 KO mice}

In the classical and pancreas-specific conditional Pax6 KO mice as well as in the $\mathrm{Pax}^{\text {sey/sey }}$ mutant mice, the number of ghrelin ${ }^{+}$cells is highly elevated and that of insulin ${ }^{+}$ cells is decreased (Ashery-Padan et al., 2004; Heller et al., 2005; Kordowich et al., 2011). In the adult beta-cell-specific Pax6 KO mice we also found an increase in the ghrelin ${ }^{+}$cell population and a decrease in the insulin $^{+}$cell population. This change was, however, gradual. Following tamoxifen induction, there was a steady increase in the number of ghrelin $^{+}$cells (Figure 3.3a and 3.4) with a concomitant decrease in the number of insulin ${ }^{+}$ cells over time (Figure 3.3a and 3.5). This inverse relationship over time indicated a possible direct conversion of one cell type into the other. A decrease in the expression of insulin and increase in the expression of ghrelin was also confirmed by qRT-PCR (Figure 3.3b,c). The number of other endocrine cell types, including glucagon, somatostatin, and PP producing cells, was increased in the pancreata of beta-cell-specific Pax6 KO mice (Figure 3.6).

\subsubsection{Expression of beta-cell related transcription factors in the pancreata of beta- cell-specific Pax6 KO mice}

Apart from Pax6, several other transcription factors are involved in the maintenance of mature beta-cell function. By immunofluorescence staining, we checked the expression of beta-cell related transcription factors MafA, Pdx1, Nkx6.1, Isl1, and Rfx6. MafA, Pdx1, and Nkx6.1 are specifically expressed in mature beta-cells while Isl1 and Rfx6 are expressed in all endocrine cells of the islet (Ahlgren et al., 1997; Ahlgren et al., 1998; Zhang et al., 2005; Gauthier et al., 2007; Smith et al., 2010). In beta-cell-specific Pax6 KO islets the expression of MafA was absent from most of the insulin ${ }^{+}$cells (Figure 3.7). Expression of Pdx1, Isl1 and Rfx6 was not affected in the KO islets (Figure 3.7 and 3.8). Expression of Nkx6.1 was not affected in the early days after KO induction but was 


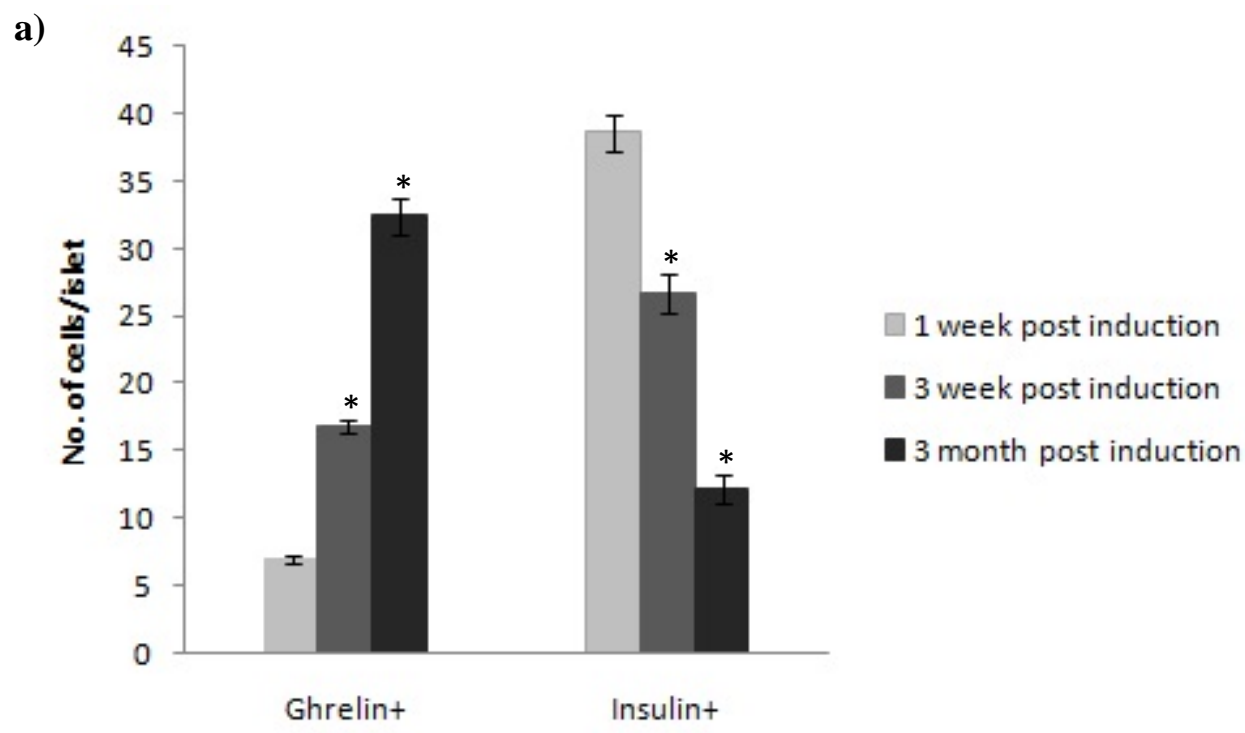

b)

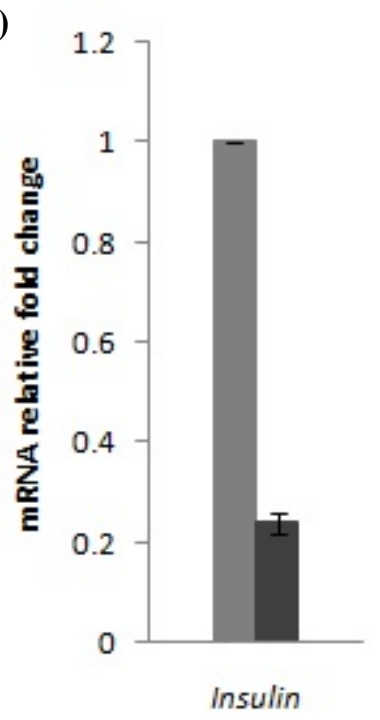

c)

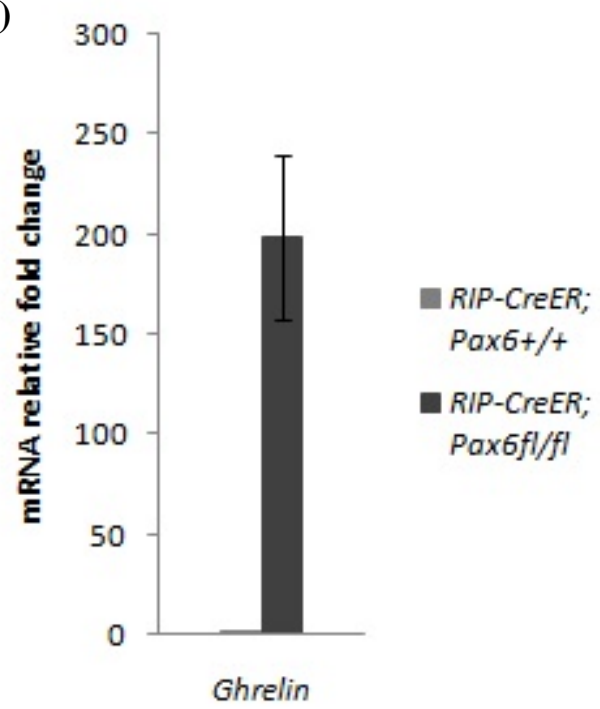

Figure 3.3: Inverse relationship of ghrelin and insullin expression in the pancreata of beta-cell-specific Pax6 KO mice. (a) Quantification of ghrelin ${ }^{+}$and insulin ${ }^{+}$cells in the islets of beta-cell-specific Pax6 KO mice (injected at 1.5 month of age) at different time points after tamoxifen induction $(n=3)$. $(b, c)$ Quantitative RT-PCR of insulin and ghrelin mRNA in the pancreata of 4.5 month old mice at 3 months after tamoxifen induction ( $\mathrm{n}=2)$. Error bars represent SEM; ${ }^{*} \mathrm{p}<0.05$. 

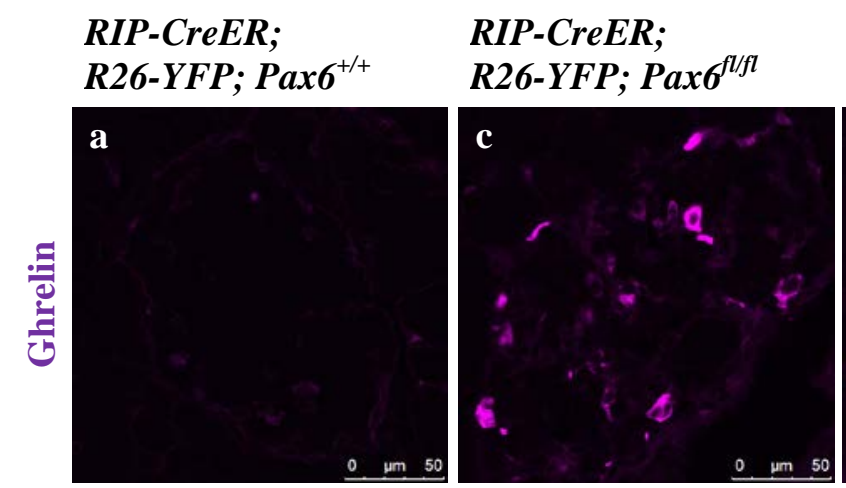

\section{RIP-CreER; R26-YFP; Pax6 $6^{f l f l}$}
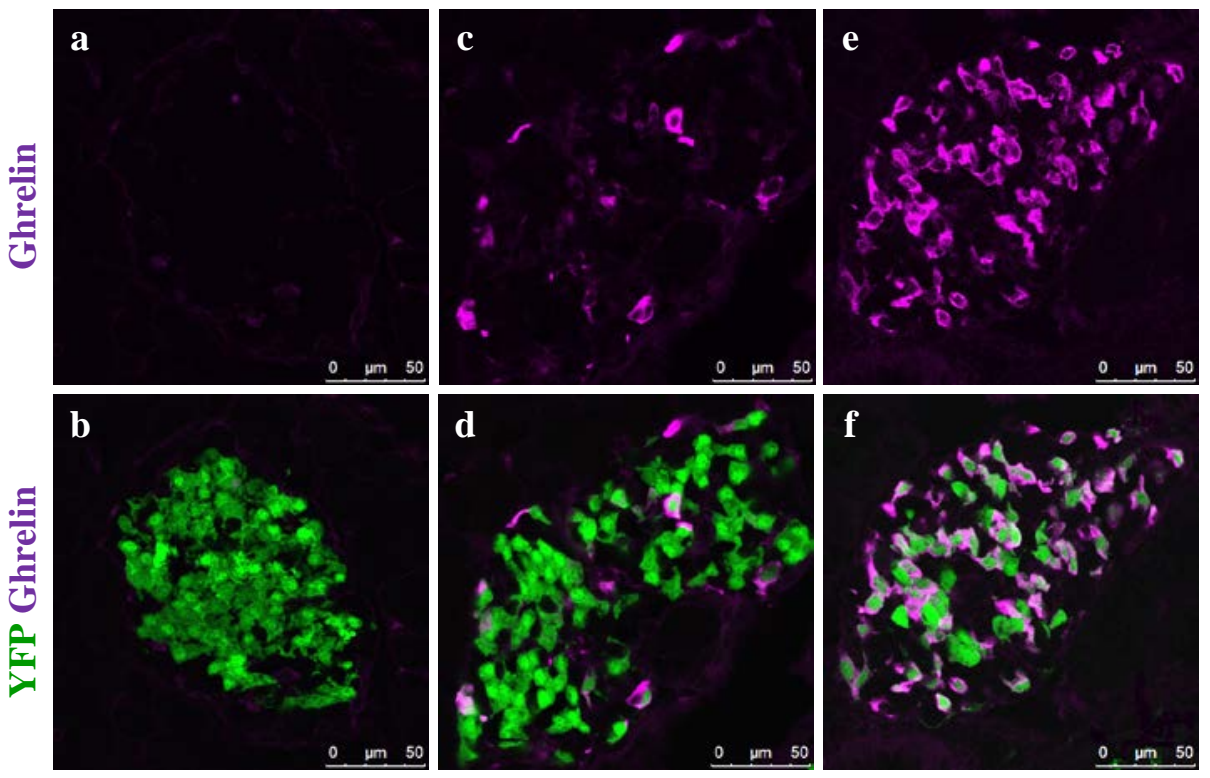

Figure 3.4: Gradual increase in the population of ghrelin expressing cells in the islets of beta-cell-specific Pax6 KO mice. Immunofluorescence staining of pancreatic cryosections from 4 week old mice at 7 days after tamoxifen induction (a-d) and 9 week old mice at 6 weeks after tamoxifen induction $(e, f)$. (a,b) Ghrelin ${ }^{+}$cells are not detected in the control islets. (c,d) At 7 days after tamoxifen induction few $\mathrm{YFP}^{+}$Pax6-deficient cells express ghrelin in the KO islets. (e,f) At 6 weeks after tamoxifen induction majority of the $\mathrm{YFP}^{+}$Pax6-deficient cells express ghrelin in the KO islets. 

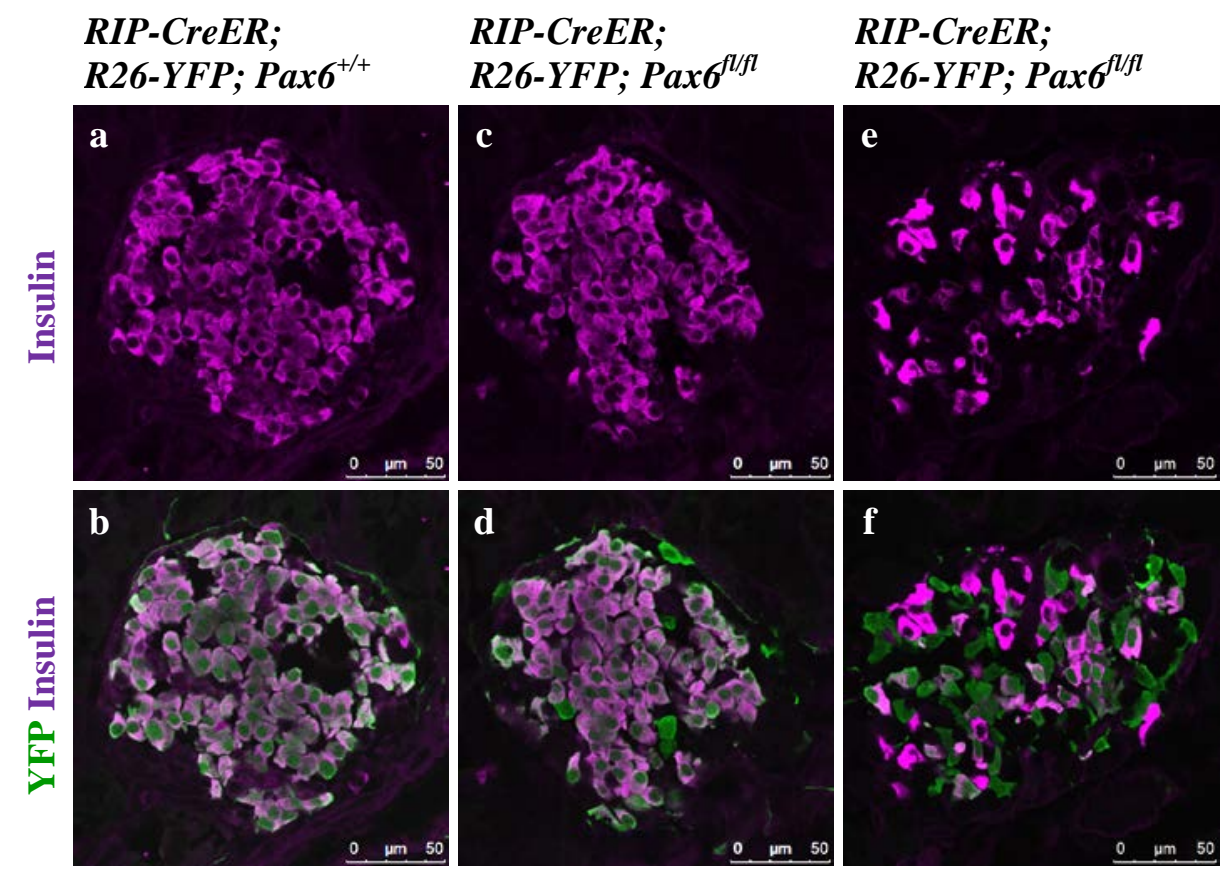

Figure 3.5: Gradual decrease in the population of insulin expressing cells in the islets of beta-cell-specific Pax6 KO mice. Immunofluorescence staining of pancreatic cryosections from 4 week old mice at 7 days after tamoxifen induction (a-d) and 9 week old mice at 6 weeks after tamoxifen induction (e, f). (a, b) In the control islets, all of the YFP $^{+}$cells express insulin. (c, d) At 7 days after tamoxifen induction few YFP $^{+}$Pax6deficient cells are negative for insulin expression. (e, f) At 6 weeks after tamoxifen induction majority of the $\mathrm{YFP}^{+}$Pax6-deficient cells are negative for insulin expression. 

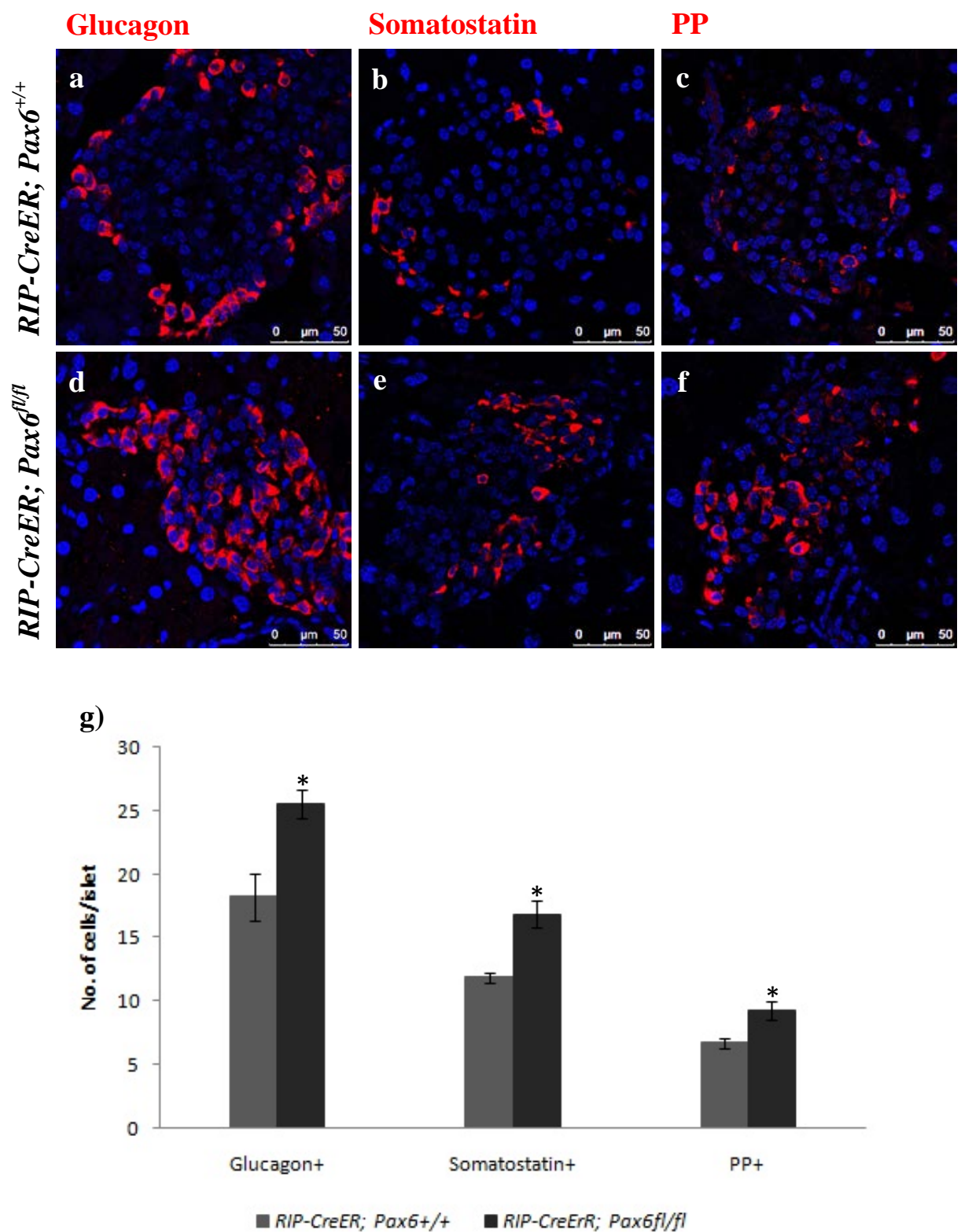

Figure 3.6: Changes in the glucagon ${ }^{+}$, somatostatin ${ }^{+}$, and $\mathrm{PP}^{+}$cell population in the islets of beta-cell-specific Pax6 KO mice. Immunofluorescence staining of pancreatic cryosections (a-f) and quantification of glucagon ${ }^{+}$, somatostatin ${ }^{+}$, and $\mathrm{PP}^{+}$cells (g) from 2 month old mice at 4 weeks after tamoxifen induction $(n=3)$. Glucagon ${ }^{+}$, somatostain ${ }^{+}$, and $\mathrm{PP}^{+}$cells are increased in number in the beta-cell-specific Pax6 KO islets. Error bars represent $\mathrm{SEM} ;{ }^{*} \mathrm{p}<0.05$. 


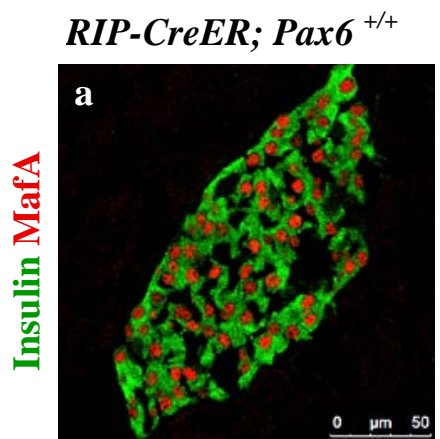

RIP-CreER; Pax6 ${ }^{\text {flfl }}$
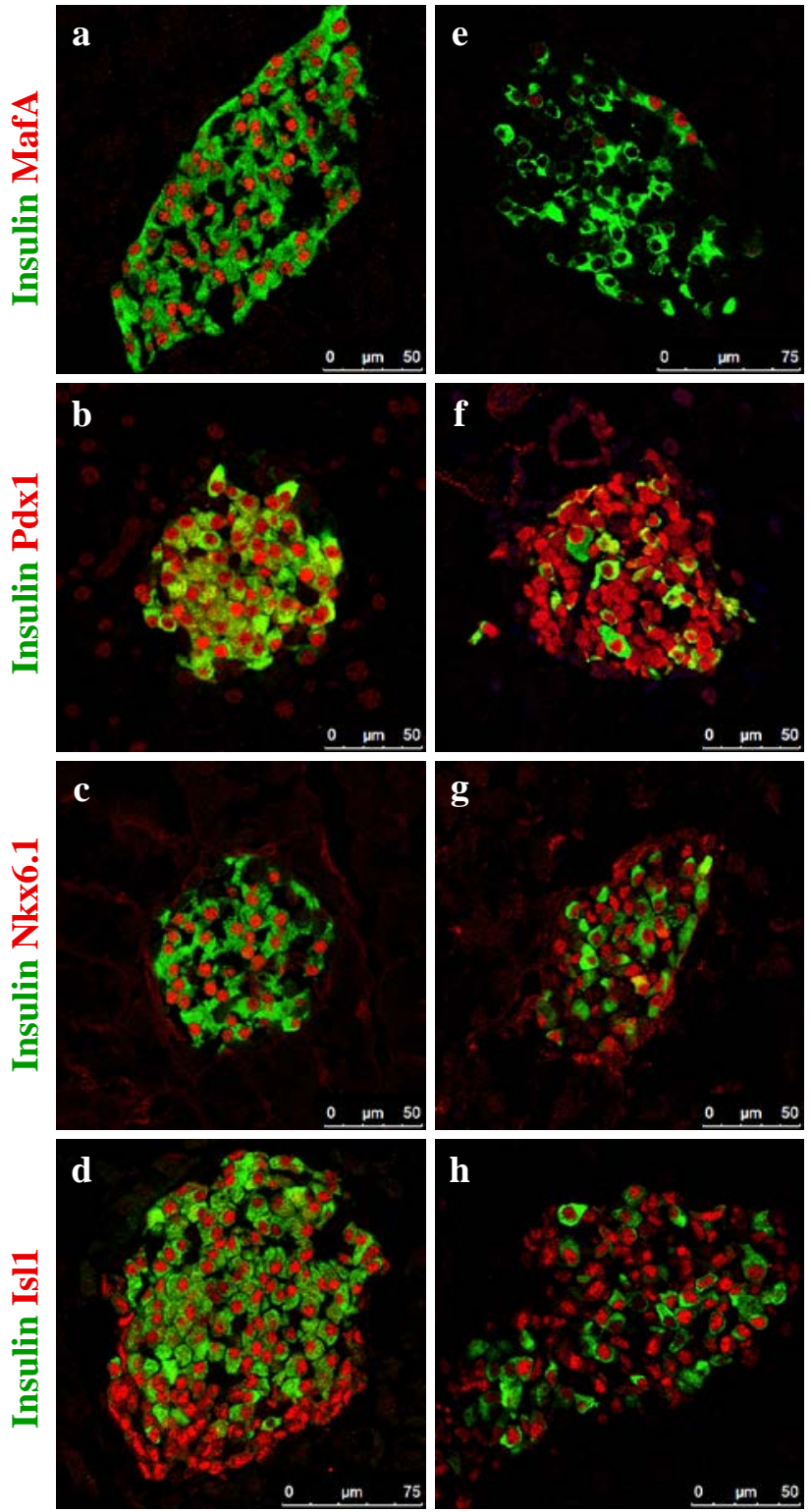

Figure 3.7: Expression of beta-cell related transcription factors in the islets of betacell-specific Pax6 KO mice. Double immunofluorescence staining of pancreatic cryosections from 2.5 month old mice at 2 weeks after tamoxifen induction. Expression of MafA is lost (e) and that of Pdx1, Nkx6.1, and Isl1 (f-h) is maintained in the islets of beta-cell-specific Pax6 KO pancreata. 


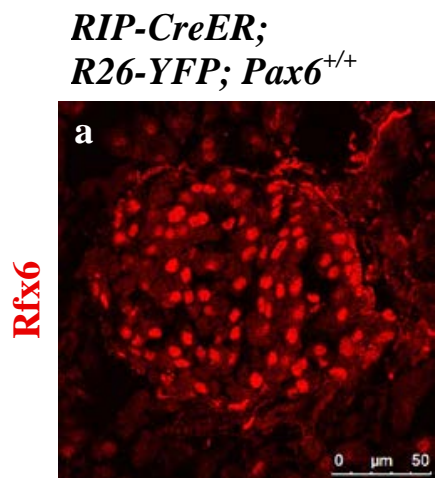

RIP-CTEER;

R26-YFP; Pax6 $^{f l f l}$
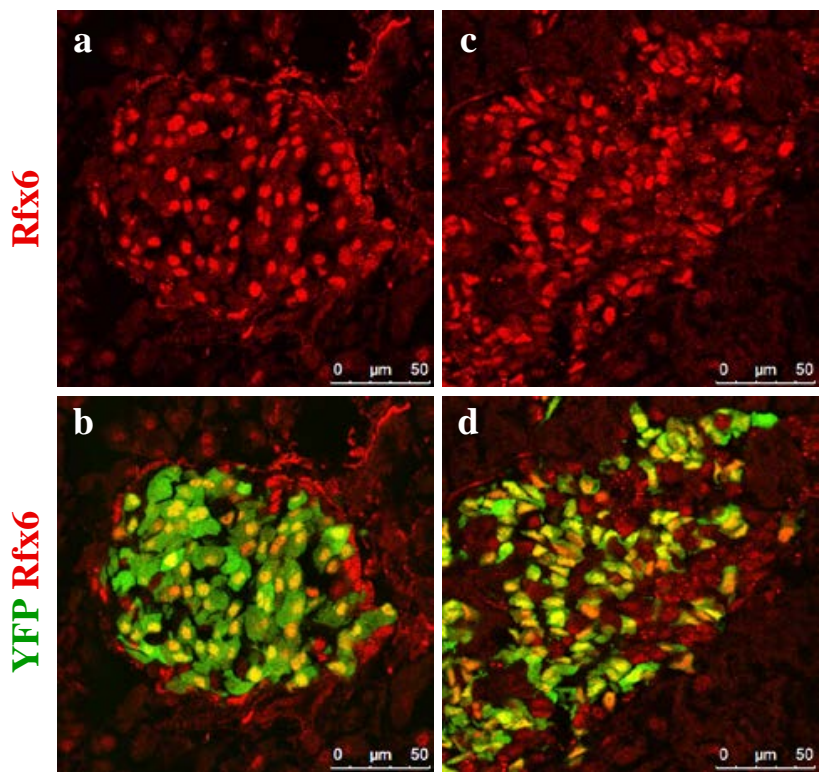

Figure 3.8: Expression of $\mathrm{Rfx} 6$ in the islets of beta-cell-specific Pax6 KO mice. Immunofluorescence staining of pancreatic cryosections from 6 month old mice at 4.5 month after tamoxifen induction. Expression of Rfx6 is maintained in the $\mathrm{YFP}^{+}$Pax6deficient cells of the beta-cell-specific Pax6 KO islets (c,d).

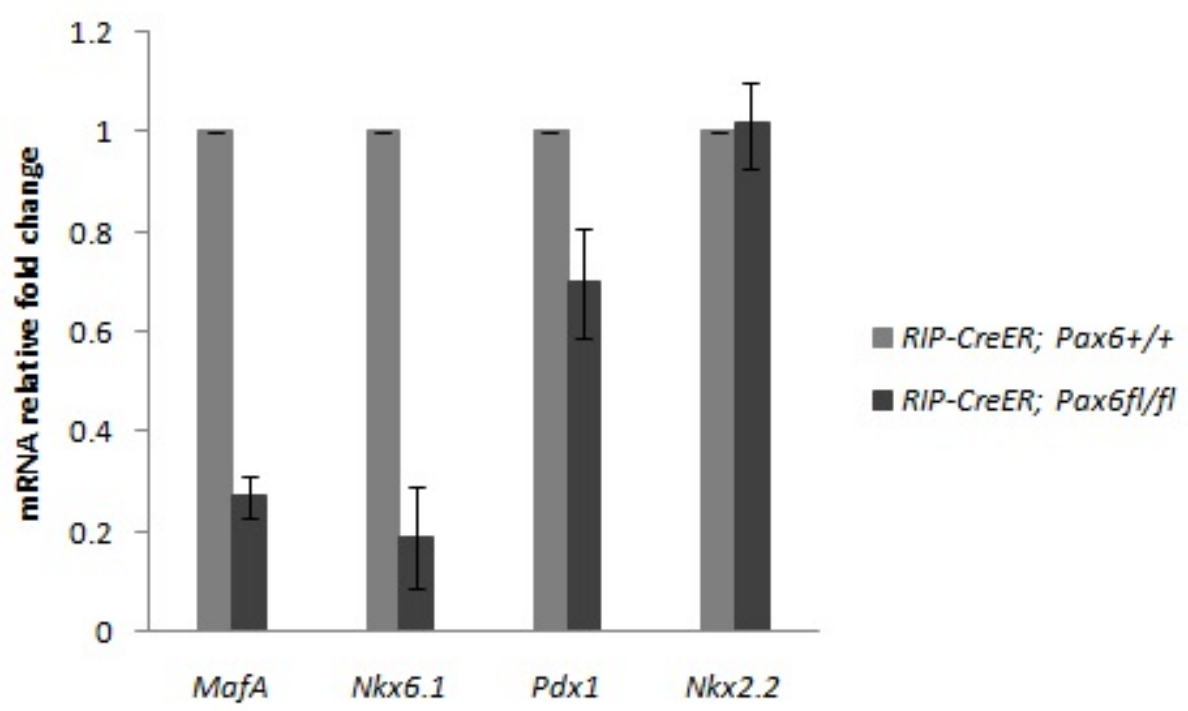

Figure 3.9: Expression of beta-cell related transcription factors in the pancreata of beta-cell-specific Pax6 KO mice. Quantitative RT-PCR of MafA, Nkx6.1, Pdx1, and $N k x 2.2$ mRNA in the pancreata of 4.5 month old mice at 3 months after tamoxifen induction ( $\mathrm{n}=2)$. Error bars represent SEM. 
reduced at the later stage (Figure 3.7 and 3.9). The expression of MafA, Pdx1, Nkx6.1, and Nkx2.2 was also checked by qRT-PCR. Here the expression of MafA and Nkx6.1 was found to be reduced, Pdx1 expression was slightly reduced and that of Nkx2.2 not affected at all (Figure 3.9).

\subsubsection{Expression of Glut2 and GLP-1 receptor is lost after ablation of Pax6 in beta- cells}

Glut2 is a glucose transporter in beta-cells that plays an important role in glucosestimulated insulin secretion. Loss of Glut2 disturbs the first phase of glucose-stimulated insulin secretion (Guillam et al., 1997). Shortly after tamoxifen induction the expression of Glut2 was found to be lost in the islets of beta-cell-specific Pax6 KO mice (Figure 3.10). Additionally, when checked in the presence of YFP reporter, Glut2 expression was found to be lost in $\mathrm{YFP}^{+}$as well as in the $\mathrm{YFP}^{-}$insulin ${ }^{+}$cell population of the $\mathrm{KO}$ islets (Figure 3.11). This indicates that the expression of Glut2 is either affected by Pax6 ablation directly and/or indirectly by the resulting hyperglycemia. Effect of hyperglycemia on Glut2 expression has been reported before (Thorens et al., 1992).

Glucagon-like peptide 1 (GLP-1) released from the intestinal L cells is known to promote insulin secretion from beta-cells in a glucose dependent manner. The effect of GLP-1 is mediated through the GLP-1 receptor that is expressed on beta-cells (MacDonald et al., 2002). In beta-cell-specific Pax6 KO islets the expression of GLP-1 receptor was lost. This loss was specific to the YFP labeled Pax6-deficient cells and a normal expression was found in the $\mathrm{YFP}^{-}$insulin $^{+}$cells (Figure 3.12). Loss of Glut2 and GLP-1 receptor expression indicates an obvious defect in the glucose-stimulated insulin secretion in the pancreata of beta-cell-specific Pax6 KO mice.

\subsubsection{Defective proinsulin processing in Pax6-deficient beta-cells}

In beta-cells insulin is synthesized as a prohormone that is cleaved by prohormone convertases 1/3 and 2 (PC1/3 and PC2) to generate mature insulin peptide and C-peptide. C-peptide is released together with insulin at an equimolar concentration (Vasic and Walcher, 2012; Goodge and Hutton, 2000). A defect of proinsulin processing can decrease the amount of C-peptide produced and leads to a concomitant increase in the amount of proinsulin. We checked the expression of PC1/3, PC2, and C-peptide in the islets of beta-cell-specific Pax6 KO mice. Expression of PC1/3 and PC2 was unchanged 

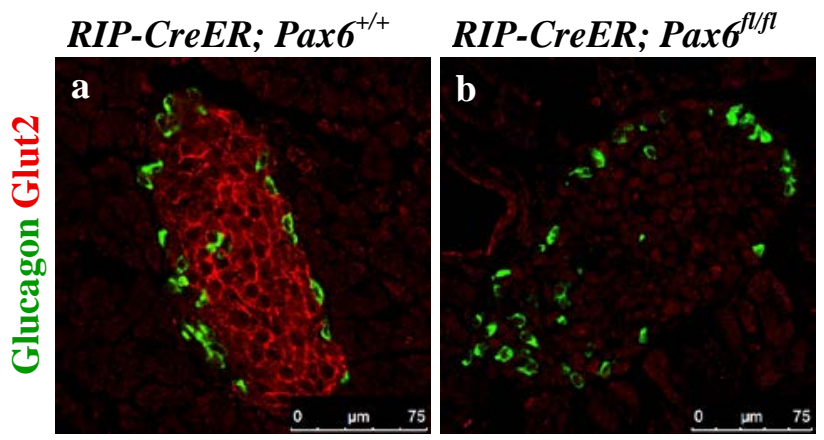

Figure 3.10: Expression of Glut2 in the islets of beta-cell-specific Pax6 KO mice. Double immunofluorescence staining of pancreatic cryosections from 2.5 month old mice at 2 weeks after tamoxifen induction. Glut2 is expressed in beta-cells of the control islets (a). In the KO pancreata Glut2 expression is lost in the entire islet (b).

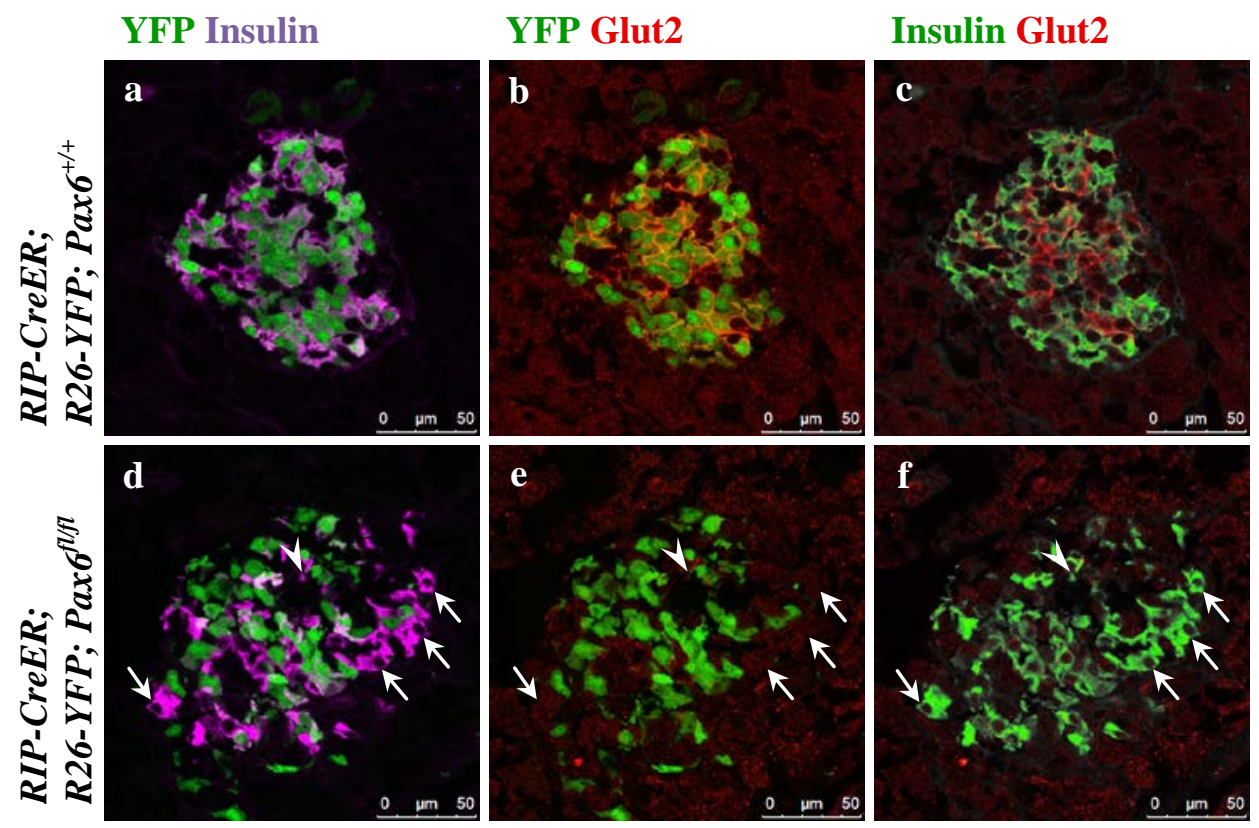

Figure 3.11: Loss of Glut2 expression due to direct and/or indirect effect of Pax6 ablation in the islets of beta-cell-specific Pax6 KO mice. Double immunofluorescence staining of pancreatic cryosections from 2 month old mice at 4 weeks after tamoxifen induction. In the control islets, expression of Glut2 is detected in both $\mathrm{YFP}^{+}$and $\mathrm{YFP}^{-}$ insulin $^{+}$cells (a-c). In the beta-cell-specific Pax6 KO islets, Glut2 expression is lost in the YFP labeled Pax6-deficient cells as well as in majority of the YFP $^{-}$insulin ${ }^{+}$cells (aroows d-f). Rarely, some YFP ${ }^{-}$insulin $^{+}$cells do express Glut2 in the KO islets (arrowhead d-f). 


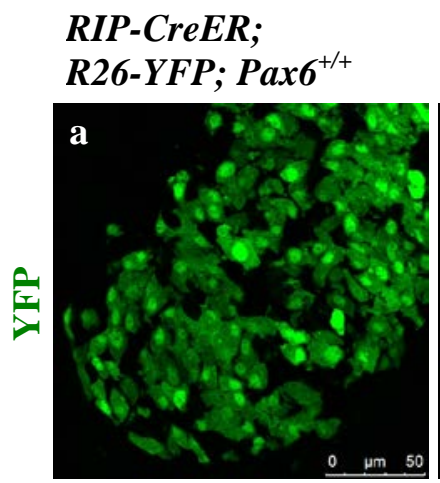

\section{RIP-CreER; R26-YFP; Pax $^{f l f l}$}
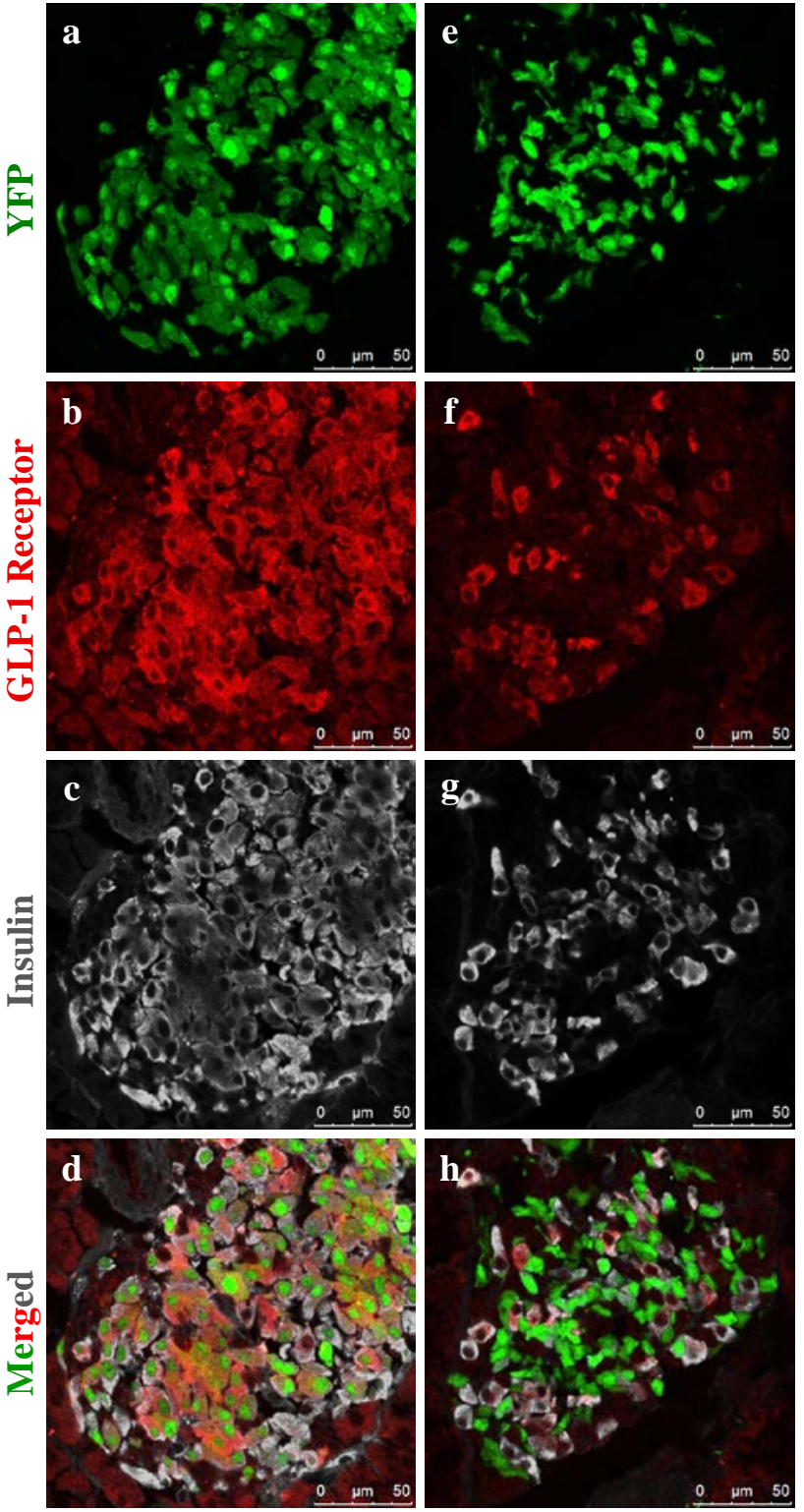

Figure 3.12: Expression of GLP-1 receptor in the islets of beta-cell-specific Pax6 KO mice. Double immunofluorescence staining of pancreatic cryosections from 4 month old mice at 6 weeks after tamoxifen induction. GLP-1 receptor is expressed in both $\mathrm{YFP}^{+}$and $\mathrm{YFP}^{-}$insulin ${ }^{+}$cells in the control islets (a-d). In the beta-cell-specific Pax6 KO islets, GLP-1 receptor expression is lost from the YFP labeled Pax6-deficient cells but maintained in the $\mathrm{YFP}^{-}$insulin ${ }^{+}$cells (e-h). 
in the Pax6-deficient YFP labeled cells including those cells that had even stopped the expression of insulin (Figure 3.13). Interestingly, however, the expression of C-peptide was lost in the Pax6-deficient YFP labeled cells but was maintained in the YFP $^{-}$insulin $^{+}$ cells (Figure 3.14). This may indicate a defect in the normal processing of proinsulin that is not primarily due to an altered expression of prohormone convertases.

To point out the cause of defective proinsulin processing, we then checked the expression of proSAAS and 7B2. ProSAAS and 7B2 are the regulatory peptides of PC1/3 and PC2 respectively. proSAAS has been shown to inhibit the activity of PC1/3 and 7B2 has been shown to increase the activity of PC2 (Helwig et al., 2011; Liu et al., 2012). We found that the expression of both proSAAS and 7B2 was highly upregulated in the Pax6deficient YFP labeled cells of the beta-cell-specific Pax6 KO islets but the expression was similar to control islets in the $\mathrm{YFP}^{-}$insulin $^{+}$cells (Figure 3.15 and 3.16). Furthermore, this increase in the expression of proSAAS and 7B2 was not immediate but gradual after the induction of Pax6 ablation. In the early days after tamoxifen induction, only few YFP labeled cells showed upregulated expression of proSAAS and 7B2. However, after long time following the tamoxifen induction nearly all of the YFP labeled cells expressed high levels of proSAAS and 7B2 (Figure 3.17).

Therefore, the defective proinsulin processing in Pax6-deficient beta-cells is mainly due to the increased level of proSAAS that would inhibit the activity of PC1/3.

\subsubsection{Ghrelin ${ }^{+}$cells originate from beta-cells in the beta-cell-specific Pax6 KO mice}

Previous studies have shown that the ghrelin ${ }^{+}$cell population is expanded in the Pax6 KO and Pax6 $6^{\text {sey/sey }}$ pancreata (Heller et al., 2005; Kordowich et al., 2011). This led to the conclusion that Pax6 somehow antagonizes the development of ghrelin ${ }^{+}$cells. As compared to the adult pancreas, ghrelin ${ }^{+}$cells are normally present at a higher number in the embryonic pancreas and further occur as a ghrelin only or ghrelin-glucagon double positive cell population (Prado et al., 2004). This makes it hard to exactly define the origin of upregulated ghrelin ${ }^{+}$cells in the embryonic pancreas of Pax6 KO mice. Furthermore, as the Pax6 KO mice die shortly after birth (St-Onge et al., 1997; AsheryPadan et al., 2004), it makes it impossible to analyze the ghrelin ${ }^{+}$cells over a long period of time. 


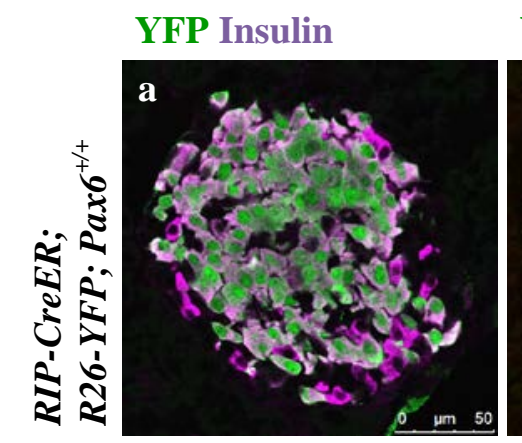

YFP PC1/3

Insulin PC1/3
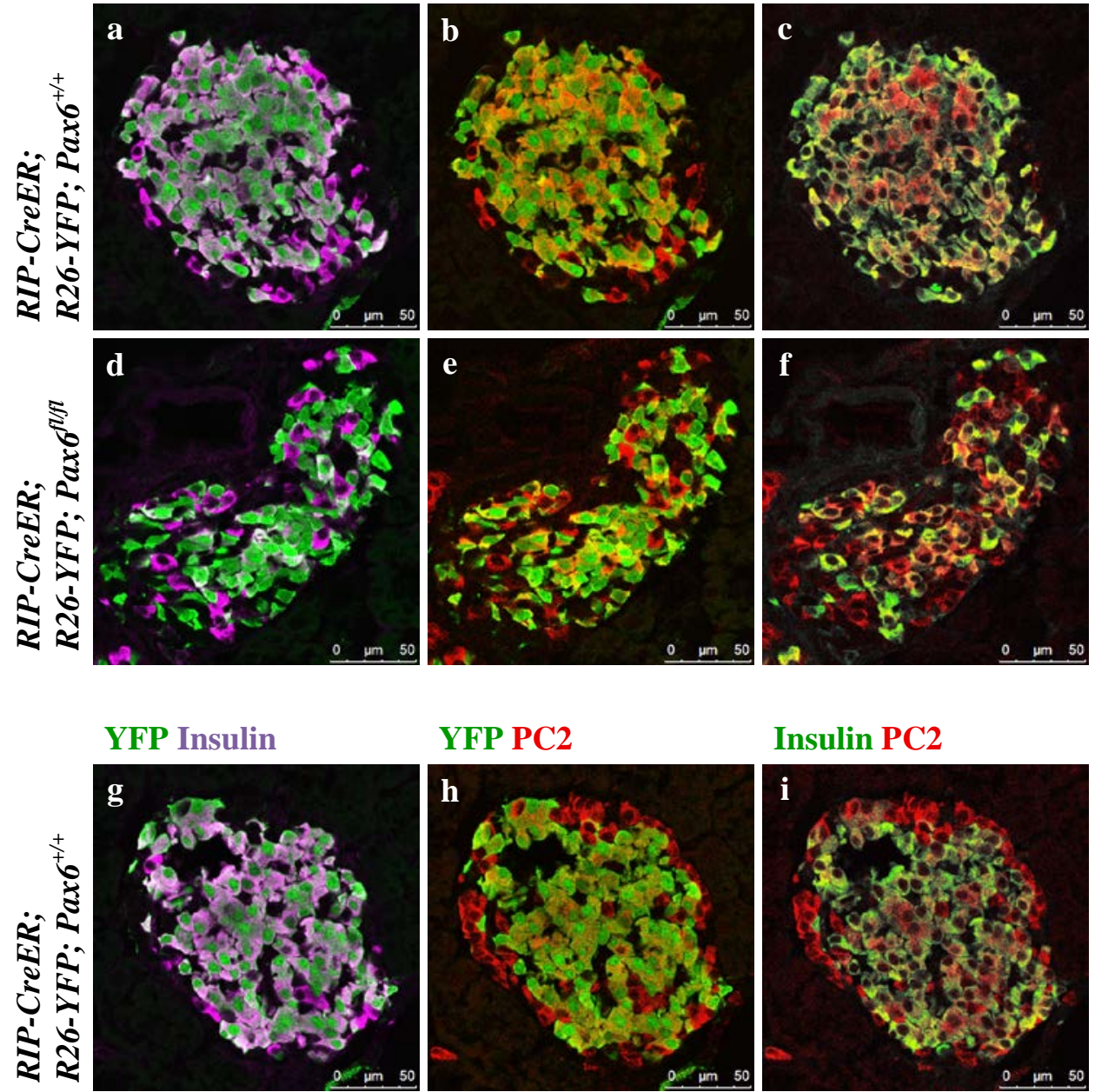

Insulin PC2
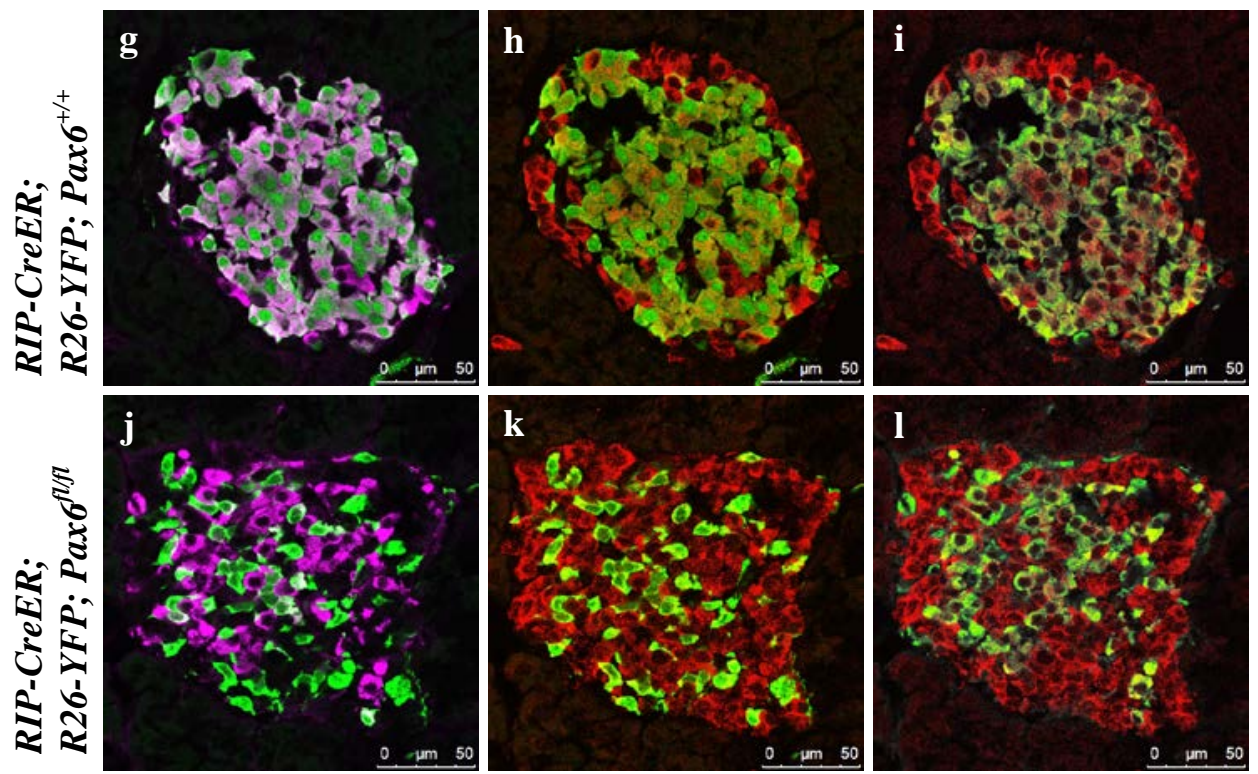

Figure 3.13: Expression of PC1/3 and PC2 in the islets of beta-cell-specific Pax6 KO mice. Double immunofluorescence staining of pancreatic cryosections from 2 month old mice at 4 weeks after tamoxifen induction. In the control islets, expression of PC1/3 (a-c) and PC2 (g-i) is detected in both $\mathrm{YFP}^{+}$and $\mathrm{YFP}^{-}$insulin ${ }^{+}$cells. In the beta-cell-specific Pax6 KO islets, PC1/3 (d-f) and PC2 (j-l) expression is maintained in the YFP labeled Pax6-deficient cells, including those cells that have lost the expression of insulin. 


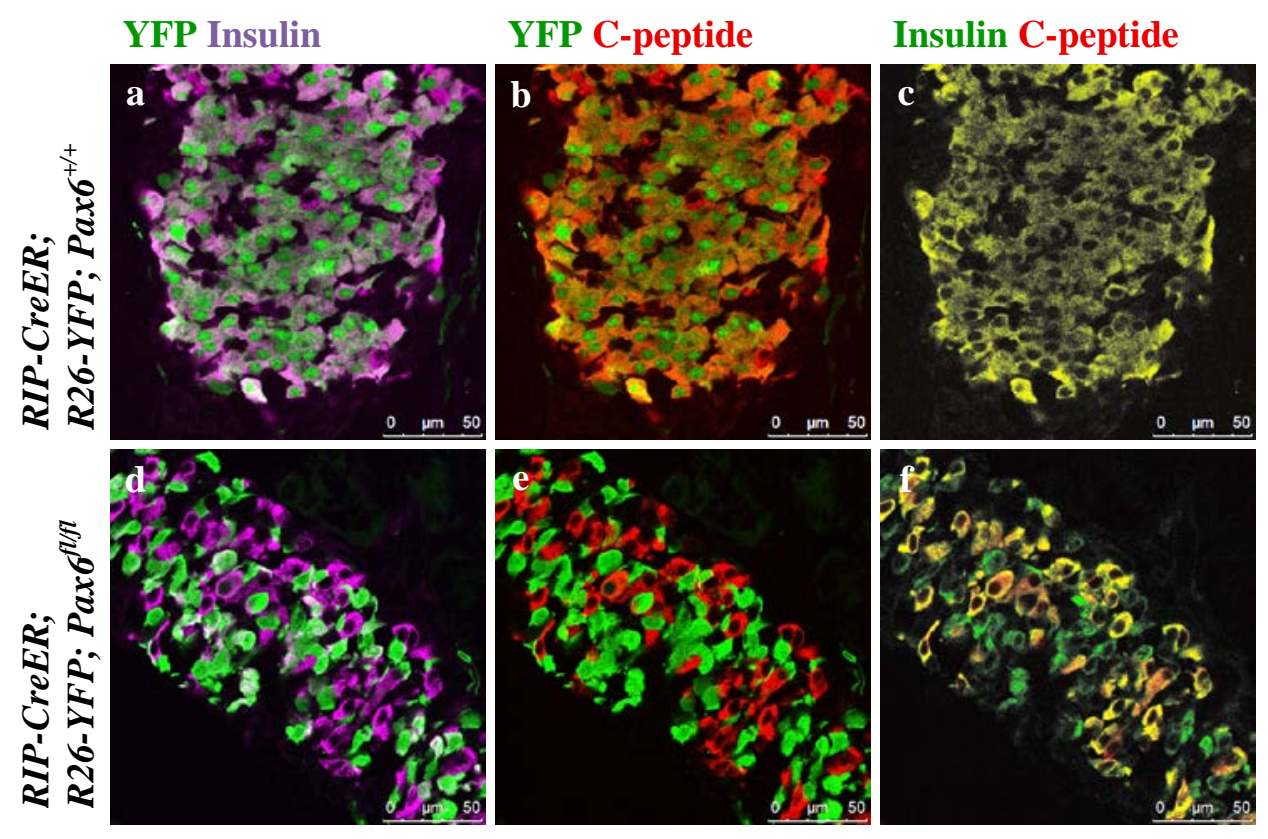

Figure 3.14: Expression of C-peptide in the islets of beta-cell-specific Pax6 KO mice. Double immunofluorescence staining of pancreatic cryosections from 2 month old mice at 4 weeks after tamoxifen induction. In the control islets, expression of C-peptide is detected in both $\mathrm{YFP}^{+}$and $\mathrm{YFP}^{-}$insulin ${ }^{+}$cells (a-c). In the beta-cell-specific Pax6 KO islets, C-peptide expression is lost from the YFP labeled Pax6-deficient cells but maintained in the $\mathrm{YFP}^{-}$insulin ${ }^{+}$cells (d-f). 


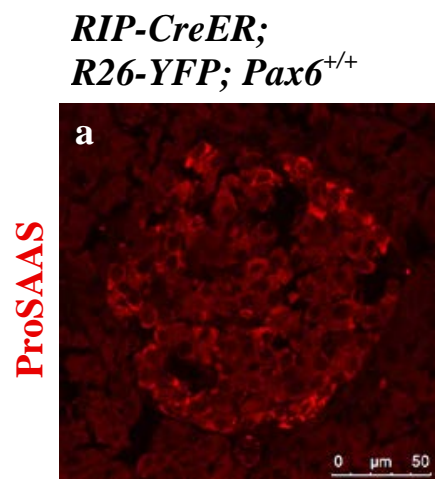

RIP-CreER; R26-YFP; Pax $^{f l / f l}$
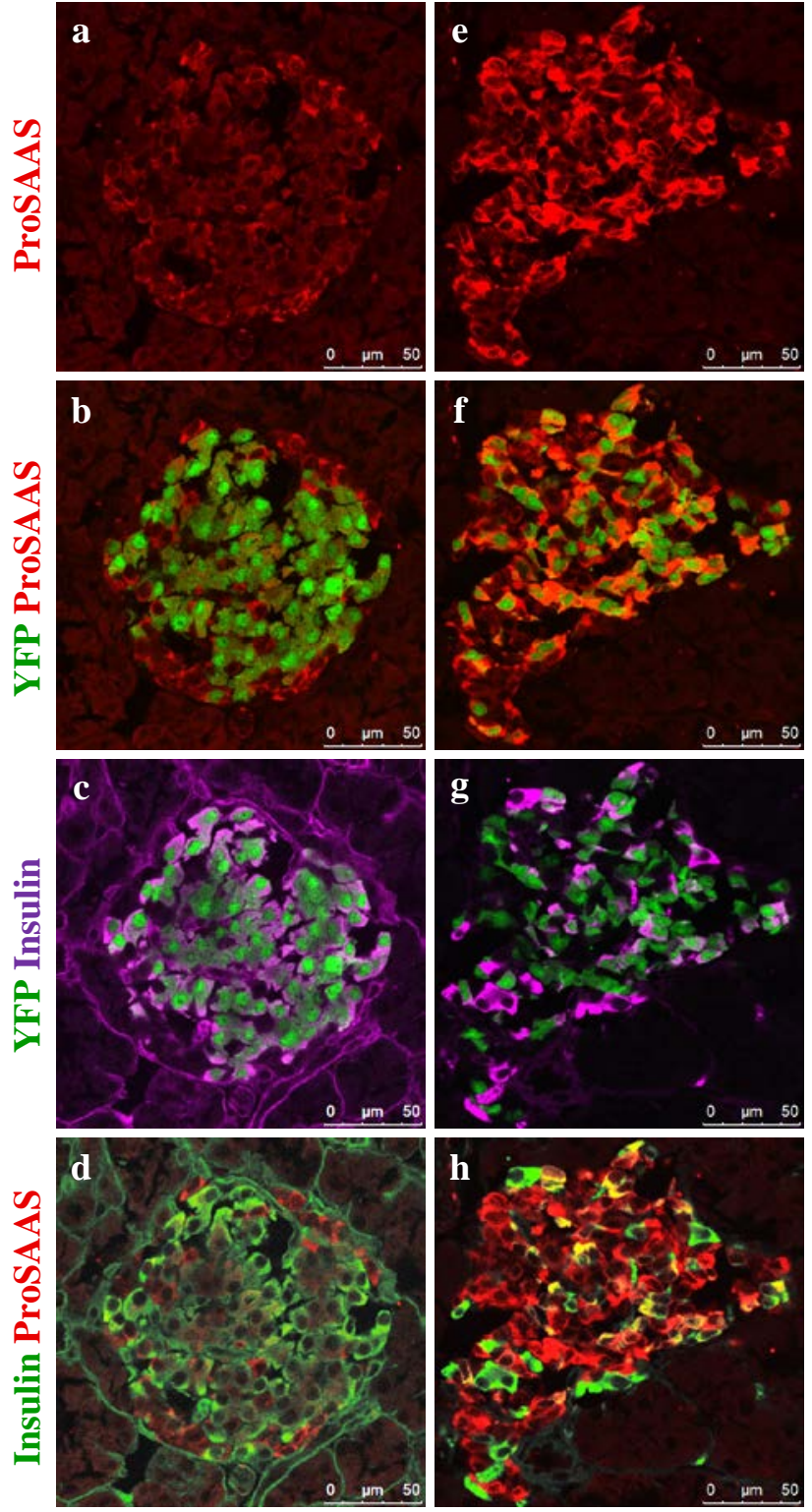

Figure 3.15: Expression of proSAAS in the islets of beta-cell-specific Pax6 KO mice. Double immunofluorescence staining of pancreatic cryosections from 4 month old mice at 6 weeks after tamoxifen induction. In the control islets, expression of proSAAS is low in the insulin ${ }^{+}$cells (a-d). In the beta-cell-specific Pax6 KO islets, expression of proSAAS is highly upregulated in the YFP labeled Pax6-deficient cells (e,f) but remains low in the $\mathrm{YFP}^{-}$insulin ${ }^{+}$cells (g,h). 


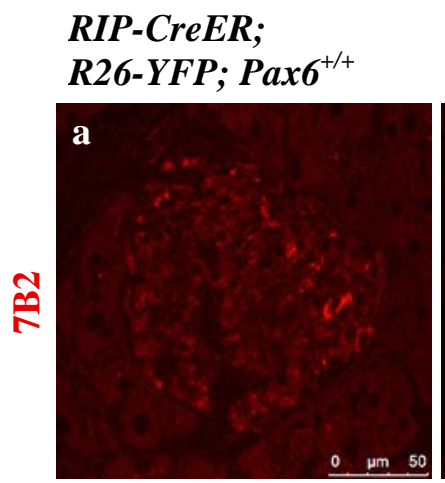

RIP-CreER; R26-YFP; Pax6 $6^{f l / f l}$
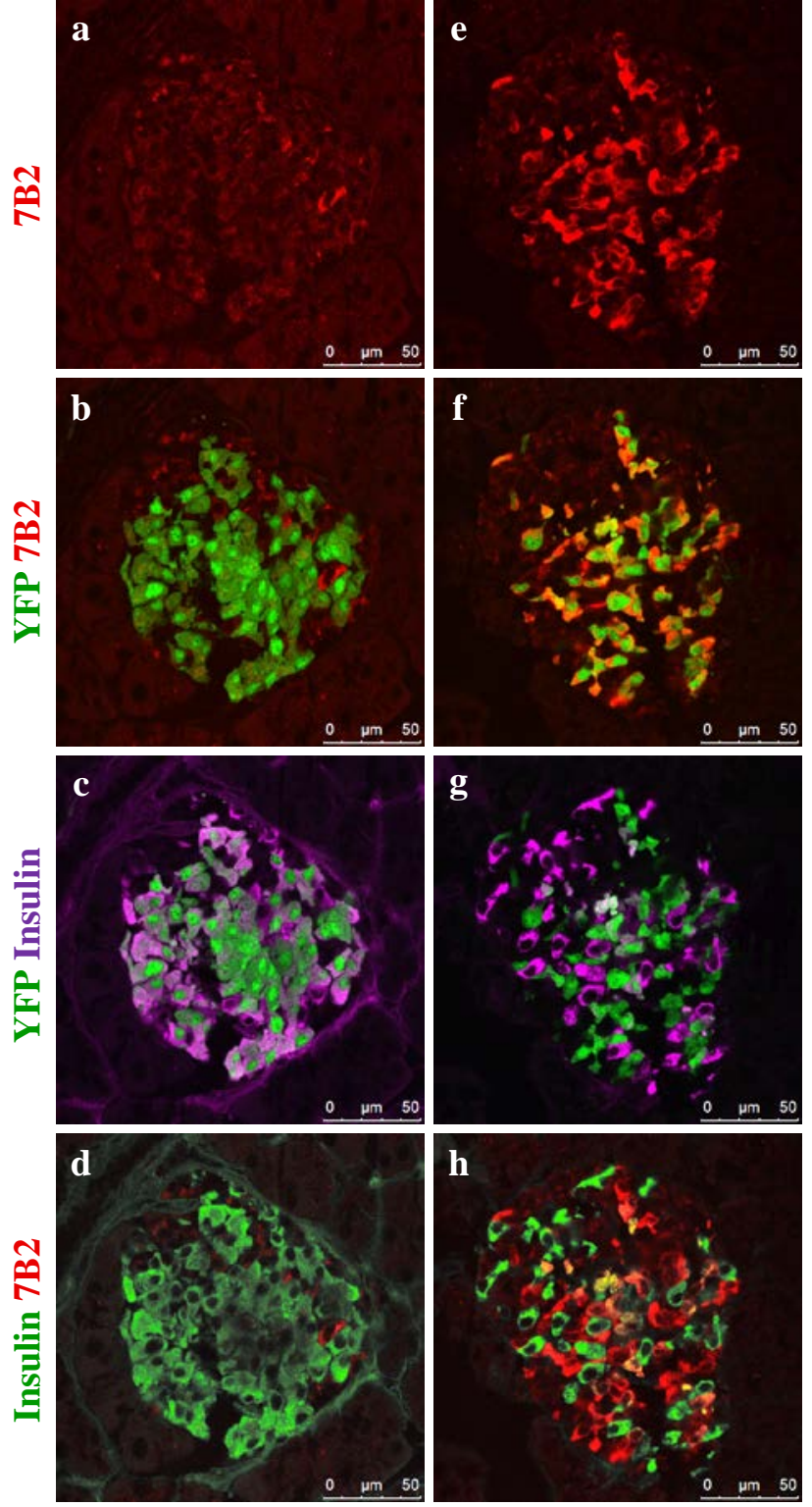

Figure 3.16: Expression of $7 \mathrm{~B} 2$ in the islets of beta-cell-specific Pax6 KO mice. Double immunofluorescence staining of pancreatic cryosections from 4 month old mice at 6 weeks after tamoxifen induction. In the control islets, expression of 7B2 is low in the insulin $^{+}$cells (a-d). In the beta-cell-specific Pax6 KO islets, expression of 7B2 is highly upregulated in the YFP labeled Pax6-deficient cells (e,f) but remains low in the YFP' insulin $^{+}$cells (g,h). 

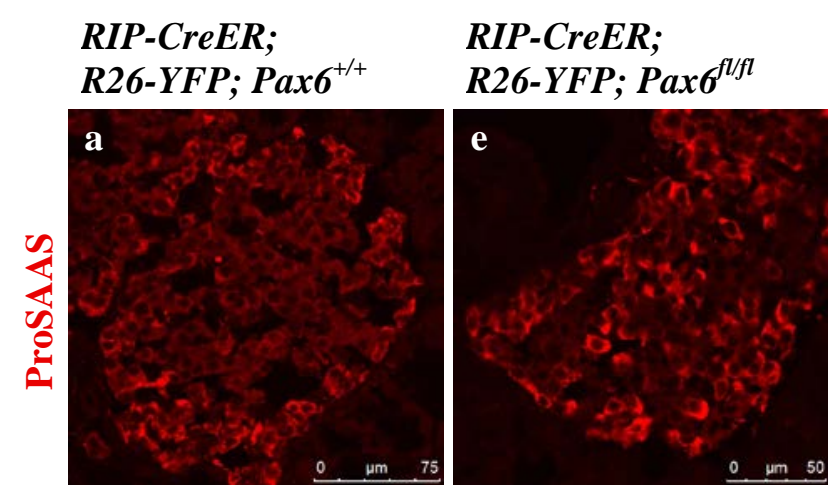

\section{RIP-CTEER; \\ R26-YFP; Pax6 $6^{f l f l}$}
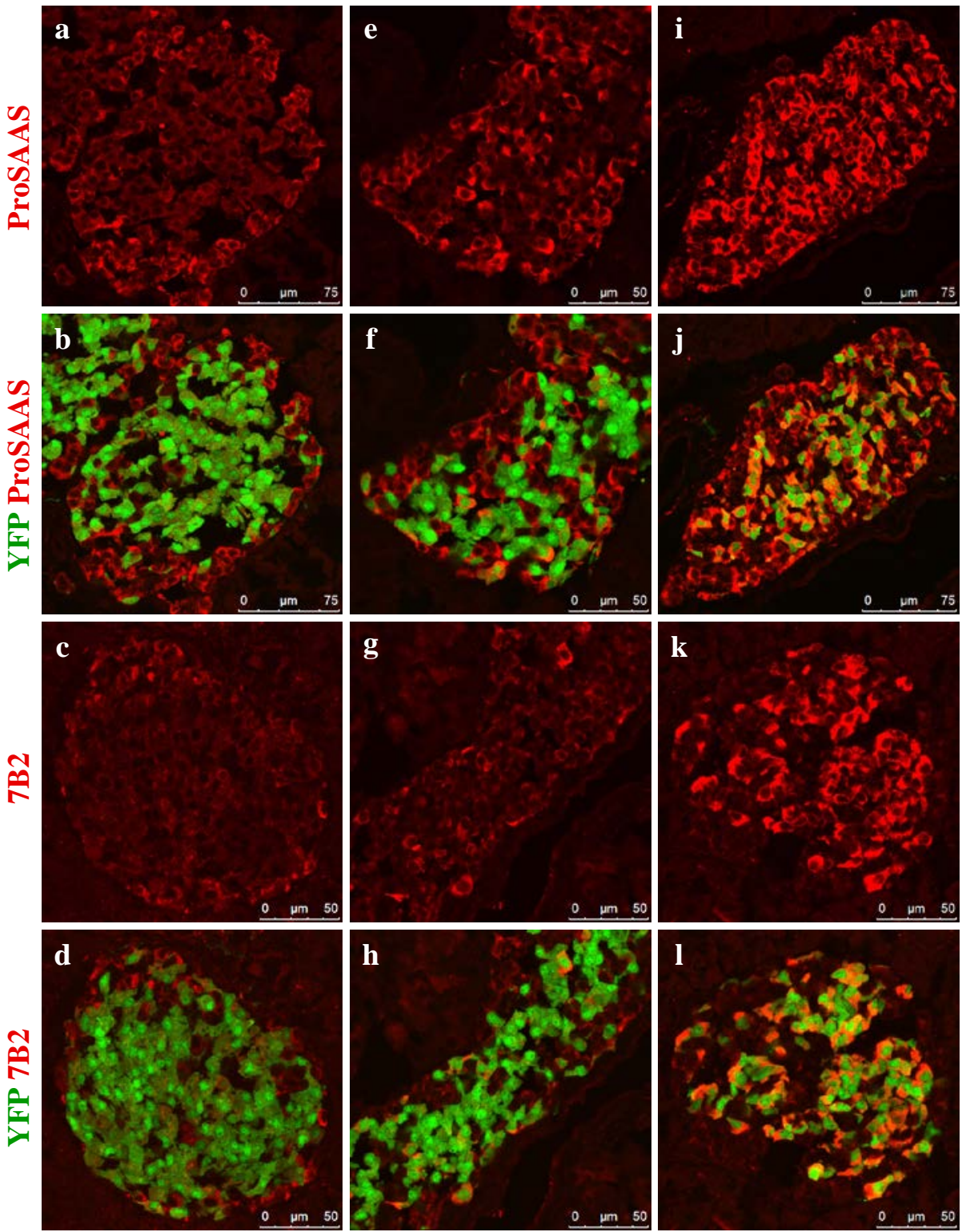

Figure 3.17: Gradual increase in the population of proSAAS and 7B2 expressing cells in the islets of beta-cell-specific Pax6 KO mice. Immunofluorescence staining of pancreatic cryosections from 4 week old mice at 7 days after tamoxifen induction (a-h) and 9 week old mice at 6 weeks after tamoxifen induction (i-l). Expression of proSAAS $(\mathrm{a}, \mathrm{b})$ and 7B2 (c,d) is very low in the YFP labeled beta-cells of the control islets. At 7 days after tamoxifen induction few YFP labeled Pax6-deficient cells start to express proSAAS (e,f) and 7B2 (g,h) at a higher level in the KO islets. At 6 weeks after tamoxifen induction majority of the YFP labeled Pax6-deficient cells express high levels of proSAAS $(\mathrm{i}, \mathrm{j})$ and $7 \mathrm{~B} 2(\mathrm{k}, \mathrm{l})$ in the KO islets. 
Therefore, an important aim of generating the adult beta-cell-specific Pax6 KO mice was to analyze the ghrelin ${ }^{+}$cell population in a greater detail. Similar to the previous studies of Pax6 KO, we found an increase in the number of ghrelin ${ }^{+}$cells in the beta-cellspecific Pax6 KO islets compared to the control islets where ghrelin expression was undetectable. Double immunofluorescence staining of ghrelin with insulin showed some ghrelin-insulin co-positive cells indicating the possible origin of ghrelin ${ }^{+}$cells from insulin producing beta-cells (Figure 3.18). To confirm it further, we checked the expression of various beta-cell related factors in the ghrelin ${ }^{+}$cells. Ghrelin ${ }^{+}$cells expressed Pdx1, Nkx6.1, and Isl1 but were negative for the expression of Pax6 (Figure 3.19). Ghrelin expression was also co-localized with that of islet amyloid polypeptide (IAPP) and PC1/3 but was never co-localized with the expression of glucagon (Figure 3.20). The absence of Pax6 and the presence of several beta-cell related markers in the ghrelin $^{+}$cells, therefore, strongly indicated the direct conversion of Pax6-deficient betacells into ghrelin expressing cells. Additionally, we noticed that the expression of IAPP was completely normal in the islets of beta-cell-specific Pax6 KO mice (Figure 3.20a,d). IAPP is another peptide hormone released from beta-cells that can modify the secretion and function of insulin (Hull et al., 2004).

Lastly, we took advantage of the YFP reporter system to exactly define the origin of ghrelin $^{+}$cells in the beta-cell-specific Pax6 KO islets. As expected, all of the ghrelin expression co-localized with the YFP labeled Pax6-deficient beta-cells. Most of these $\mathrm{YFP}^{+}$ghrelin $^{+}$cells were negative for the expression of insulin but it was possible to find many cells that were ghrelin-insulin-YFP triple positive indicating the intermediate conversion state from insulin to ghrelin expressing cell (Figure 3.21d-f). Based on these observations, we concluded that the ablation of Pax6 from beta-cells results in the downregulation of insulin expression and upregulation of ghrelin expression in these cells.

\subsubsection{Tracing the fate of Pax6-deficient beta-cells over long period of time}

So far we observed that the beta-cells lose their mature phenotype upon deletion of Pax6 and start the expression of ghrelin. Now the interesting question is that what happens to these immature Pax6 deficient beta-cells over time. As mentioned before, the ghrelin expression gradually increased following the ablation of Pax6 (Figure 3.3a and 3.4). 

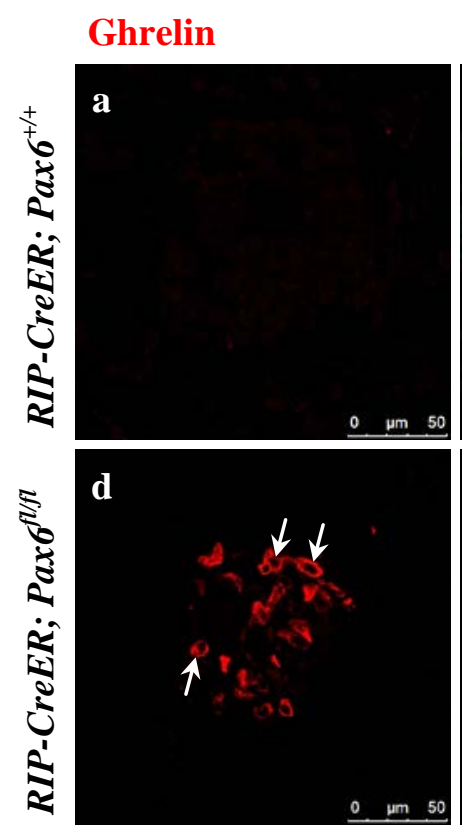

Insulin

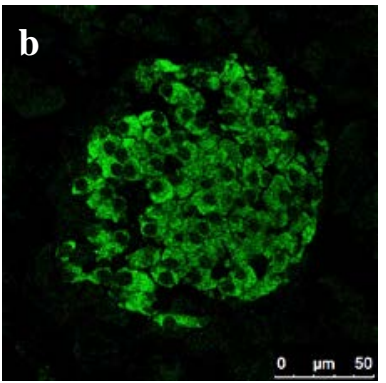

Ghrelin Insulin

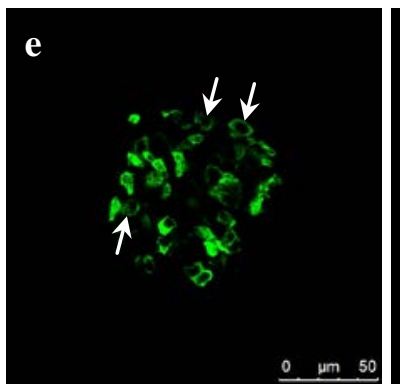

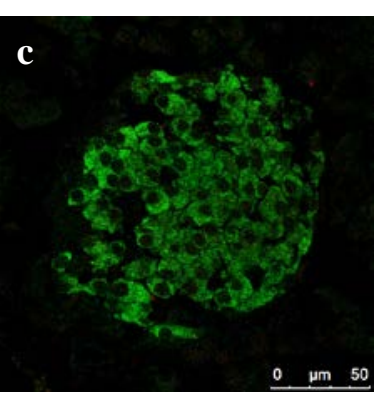

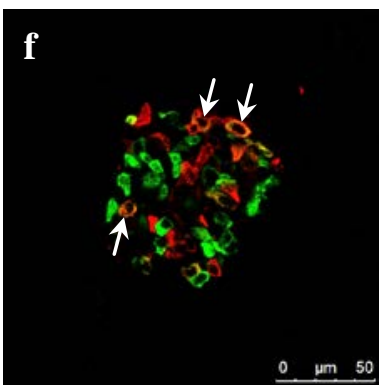

Figure 3.18: Co-expression of ghrelin with insulin in the islets of beta-cell-specific Pax6 KO mice. Double immunofluorescence staining of pancreatic cryosections from 2.5 month old mice at 2 weeks after tamoxifen induction. Ghrelin ${ }^{+}$cells are not detected in the control islets (a-c). In the beta-cell-specific Pax6 KO islets, ghrelin expression is upregulated and some of the ghrelin ${ }^{+}$cells co-express insulin (arrows d-f). 

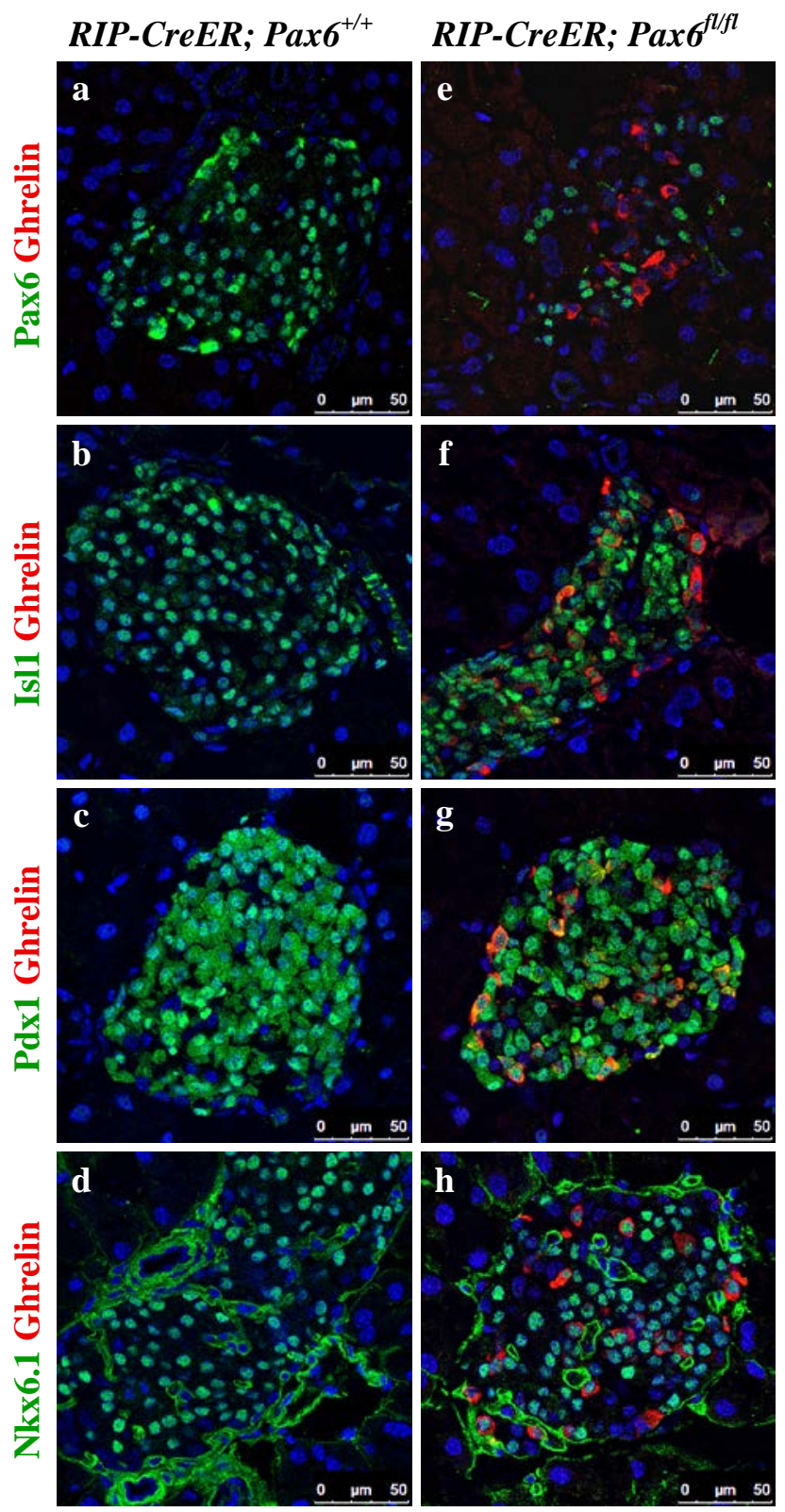

Figure 3.19: Expression of beta-cell related transcription factors in the ghrelin ${ }^{+}$cell population of the beta-cell-specific Pax6 KO islets. Double immunofluorescence staining of pancreatic cryosections from 2.5 month old mice at 2 weeks after tamoxifen induction. Ghrelin expression is not detected in the control islets (a-d). In the beta-cellspecific Pax6 KO islets, ghrelin expression is upregulated and these ghrelin ${ }^{+}$cells are negative for Pax6 (e) but positive for Isl1 (f), Pdx1 (g), and Nkx6.1 (h) expression. 


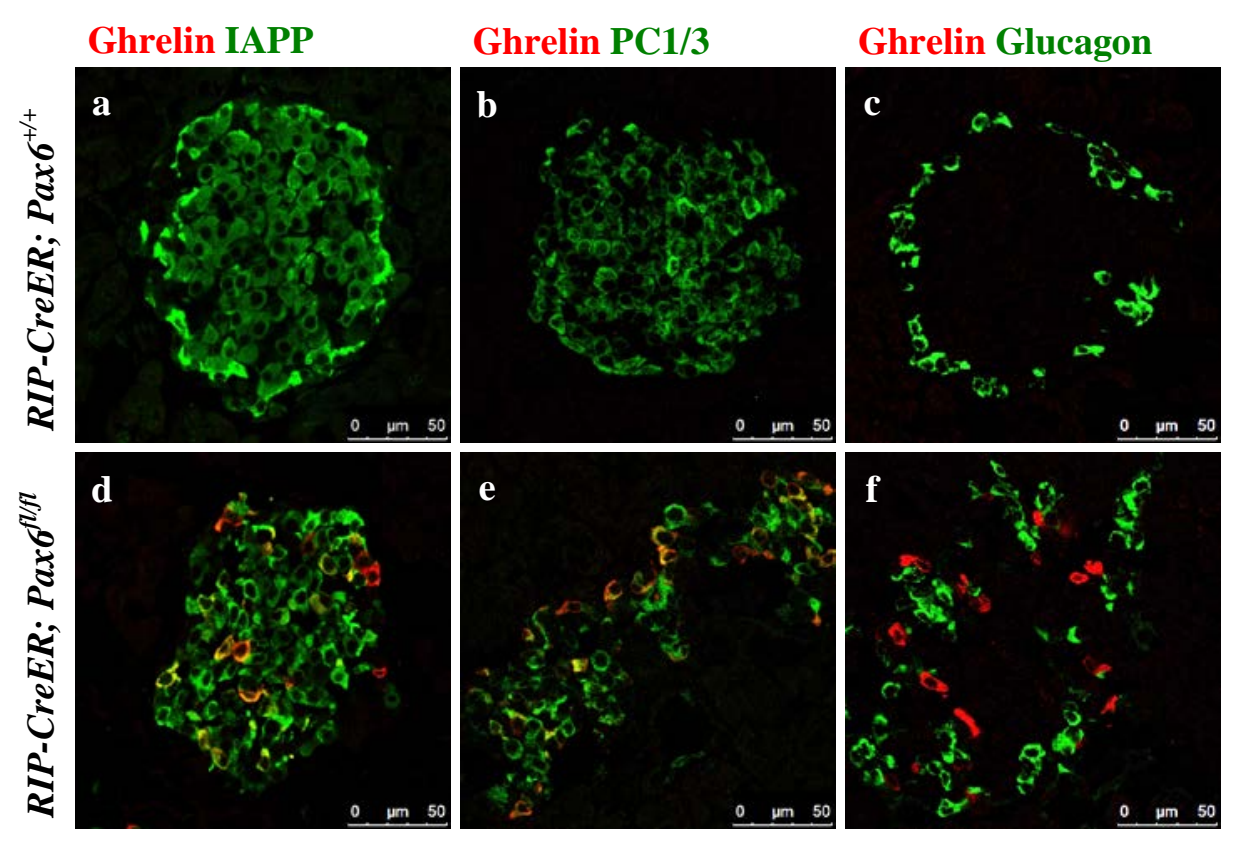

Figure 3.20: Co-expression of ghrelin with beta-cell related factors in the islets of beta-cell-specific Pax6 KO mice. Double immunofluorescence staining of pancreatic cryosections from 2.5 month old mice at 2 weeks after tamoxifen induction. Ghrelin ${ }^{+}$ cells are not detected in the control islets (a-c). In the beta-cell-specific Pax6 KO islets, ghrelin expression is upregulated and these ghrelin ${ }^{+}$cells co-express IAPP (d) and PC1/3 (e). Also note that IAPP and PC1/3 expression is maintained in the KO islets (d,e). Ghrelin $^{+}$cells never co-express glucagon in the beta-cell-specific Pax6 KO islets (f). 


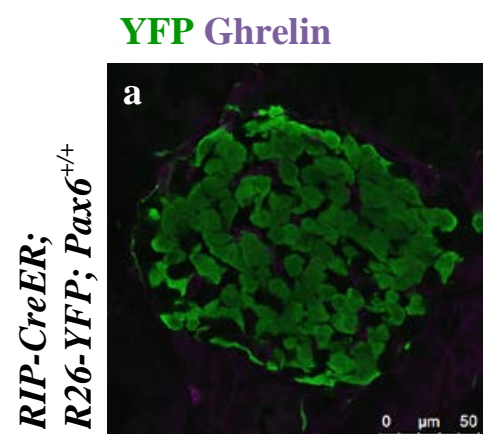

YFP Insulin
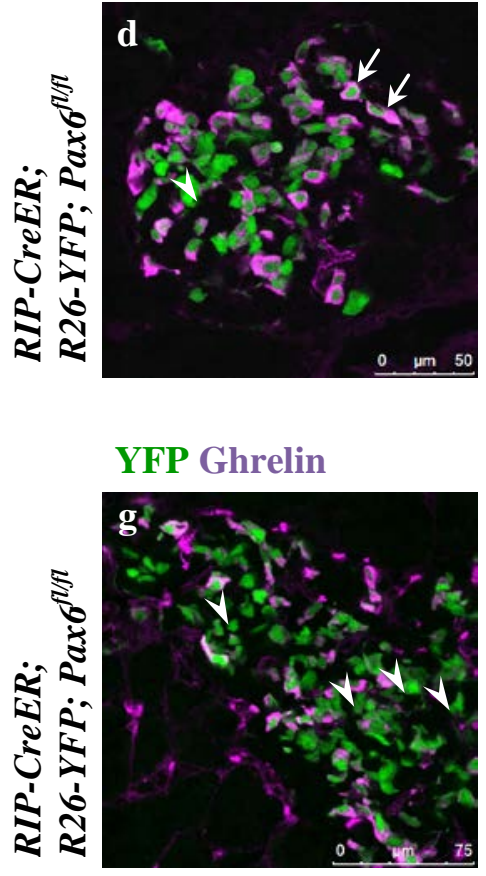
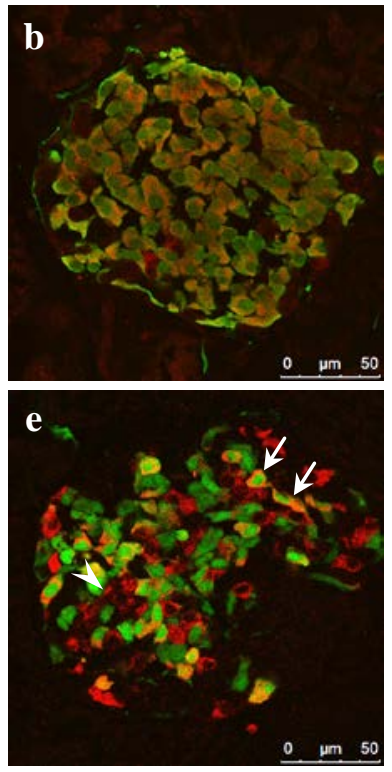

YFP Insulin

Ghrelin Insulin
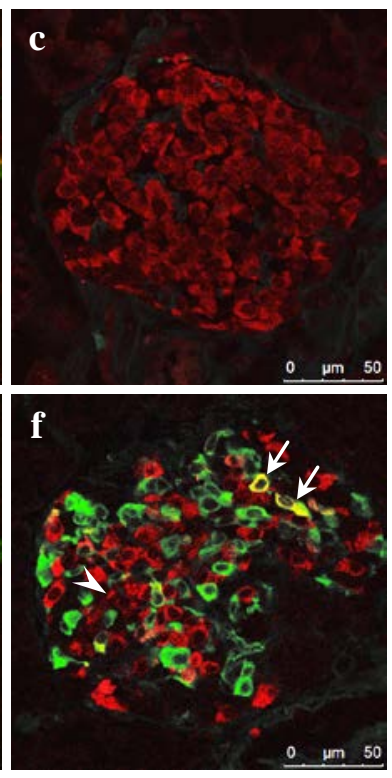

Ghrelin Insulin
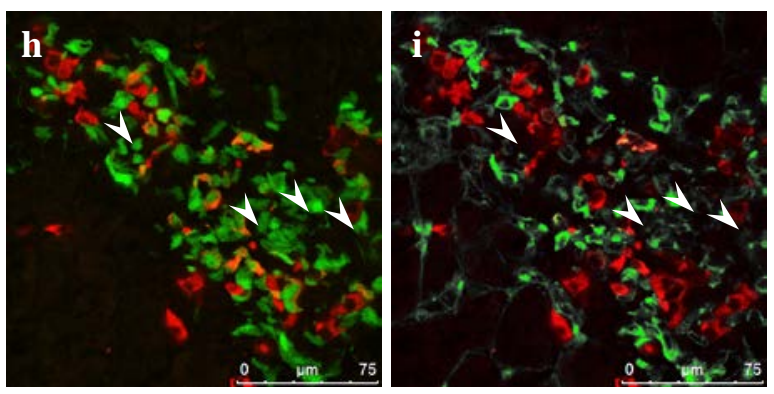

Figure 3.21: Ghrelin ${ }^{+}$cells originate from beta-cells in the beta-cell-specific Pax6 KO islets. Double immunofluorescence staining of pancreatic cryosections from 4 month old mice at 6 weeks after tamoxifen induction (a-f) and 6 month old mice at 4.5 months after tamoxifen induction (g-i). Ghrelin expression is not detected in the control islets (a-c). In the beta-cell-specific Pax6 KO islets, ghrelin expression is upregulated in the YFP labeled Pax6-deficient cells (d-f). Most of the ghrelin ${ }^{+}$cells have lost the expression of insulin but some are still insulin ${ }^{+}$(arrows d-f). Some $\mathrm{YFP}^{+}$cells in the KO islets are negative for both insulin and ghrelin (arrowheads d-f). At 4.5 months after tamoxifen induction many of the $\mathrm{YFP}^{+}$cells are negative for both insulin and ghrelin expression (arrowheads g-i). 
Quantification of $\mathrm{YFP}^{+}$ghrelin ${ }^{+}$cells further clarified that ghrelin expression is

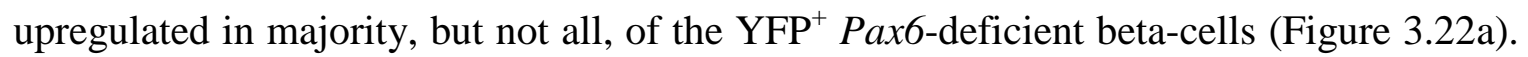
Moreover, double immunofluorescence staining with insulin and ghrelin antibodies in the presence of YFP reporter indicated a population of insulin ${ }^{-}$ghrelin ${ }^{-} \mathrm{YFP}^{+}$cells at various time points after tamoxifen induction. The number of such cells was, however, higher in the islets of those mice that were kept for the longest time-period after tamoxifen induction (upto 4.5 month) (Figure 3.21g-i and 3.22b). This indicated two things. Firstly, not all of the Pax6-deficient beta-cells upregulate ghrelin expression even after they have lost the expression of insulin. Secondly, some of the Pax6-deficient beta-cells that upregulate the expression of ghrelin, lose the high level of ghrelin expression over time.

Another possibility is that the Pax6-deficient beta-cells may change their fate to another cell type in the pancreas. However, we found that the YFP labeled cells in the beta-cell-specific Pax6 KO islets were positive for Rfx6, and Pdx1 (Figure 3.7 and 3.8) and they never co-localized with glucagon, somatostatin, or PP expressing cells (data not shown). This means that Pax6-deficient beta-cells retain their endocrine character as they are positive for Rfx6 that is an endocrine specific transcription factor in the adult pancreas (Smith et al., 2010; Soyer et al., 2010). Secondly, they do not convert to other endocrine cell types as they continue to express Pdx1 that is specific to beta-cells in the adult pancreas (Ahlgren et al., 1998).

Next, by quantification we found that the number of YFP labeled cells was always less in the beta-cell-specific Pax6 KO islets compared to the control islets when checked at few weeks post tamoxifen induction. To identify the reason behind this we checked the proliferation and apoptosis in the KO islets. By TUNEL staining we found no increased apoptosis in the KO islets (data not shown). However, we observed that the YFP labeled Pax6-deficient cells were never positive for Ki67 compared to the control islets where $\mathrm{YFP}^{+} \mathrm{Ki}^{+}$cells were frequently observed at seven weeks of age (Figure 3.23). From this we concluded that the obvious difference in the number of $\mathrm{YFP}^{+}$cells between the control and the KO islets is mainly due to the lack of proliferation in the Pax6-deficient beta-cells.

\subsubsection{Ghrelin and Glut2 are not the direct downstream targets of Pax6}

Ghrelin is upregulated and Glut2 downregulated in Pax6 KO beta-cells. To see if Pax6 directly regulates the expression of these two genes, we searched for the in silico Pax6 
a)

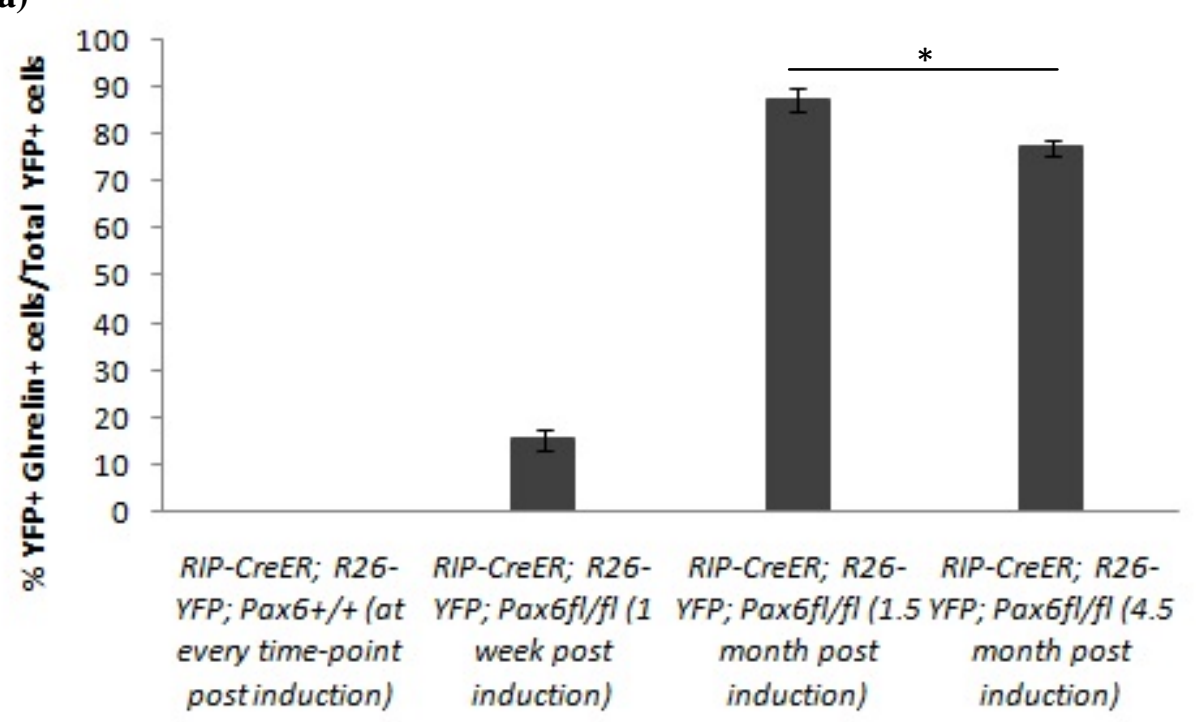

b)

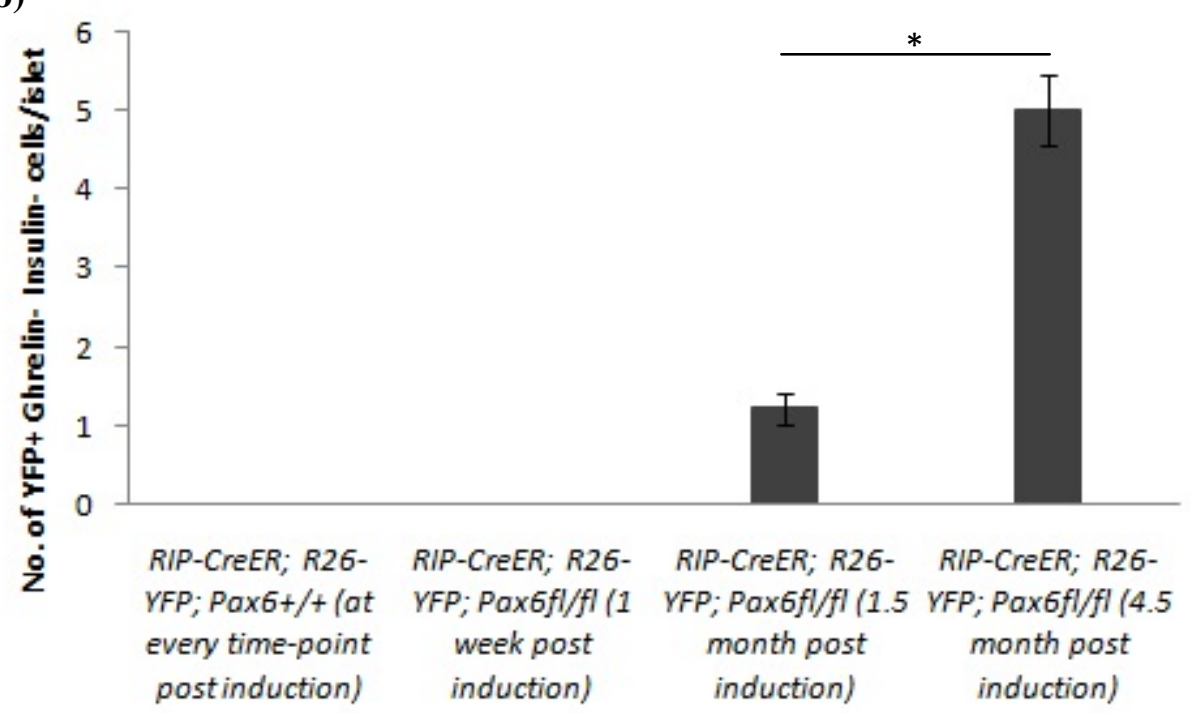

Figure 3.22: Changes in the ghrelin expressing cell population over time in the islets of beta-cell-specific Pax6 KO mice. Quantification of $\mathrm{YFP}^{+}$ghrelin ${ }^{+}$cells in relation to the total $\mathrm{YFP}^{+}$cells (a) and quantification of $\mathrm{YFP}^{+}$ghrelin ${ }^{-}$insulin ${ }^{-}$cells (b) in the islets of control and beta-cell-specific Pax6 KO mice injected with tamoxifen at 1.5 months of age and analyzed at various time points indicated $(n=3)$. (a) Ghrelin expressing cell population in the $\mathrm{KO}$ islets expands over time but ghrelin expression is never detected in $100 \%$ of $\mathrm{YFP}^{+}$Pax6-deficient cells indicating that a certain number of beta-cells do not express ghrelin after the loss of Pax6. (b) The number of $\mathrm{YFP}^{+}$ghrelin insulin cells in the $\mathrm{KO}$ islets increases over time indicating the conversion of some insulin ${ }^{+} / \mathrm{ghrelin}^{+}$or insulin-ghrelin co-positive cells to hormone negative YFP-only positive cells. Error bars represent SEM; ${ }^{*} \mathrm{p}<0.05$. 


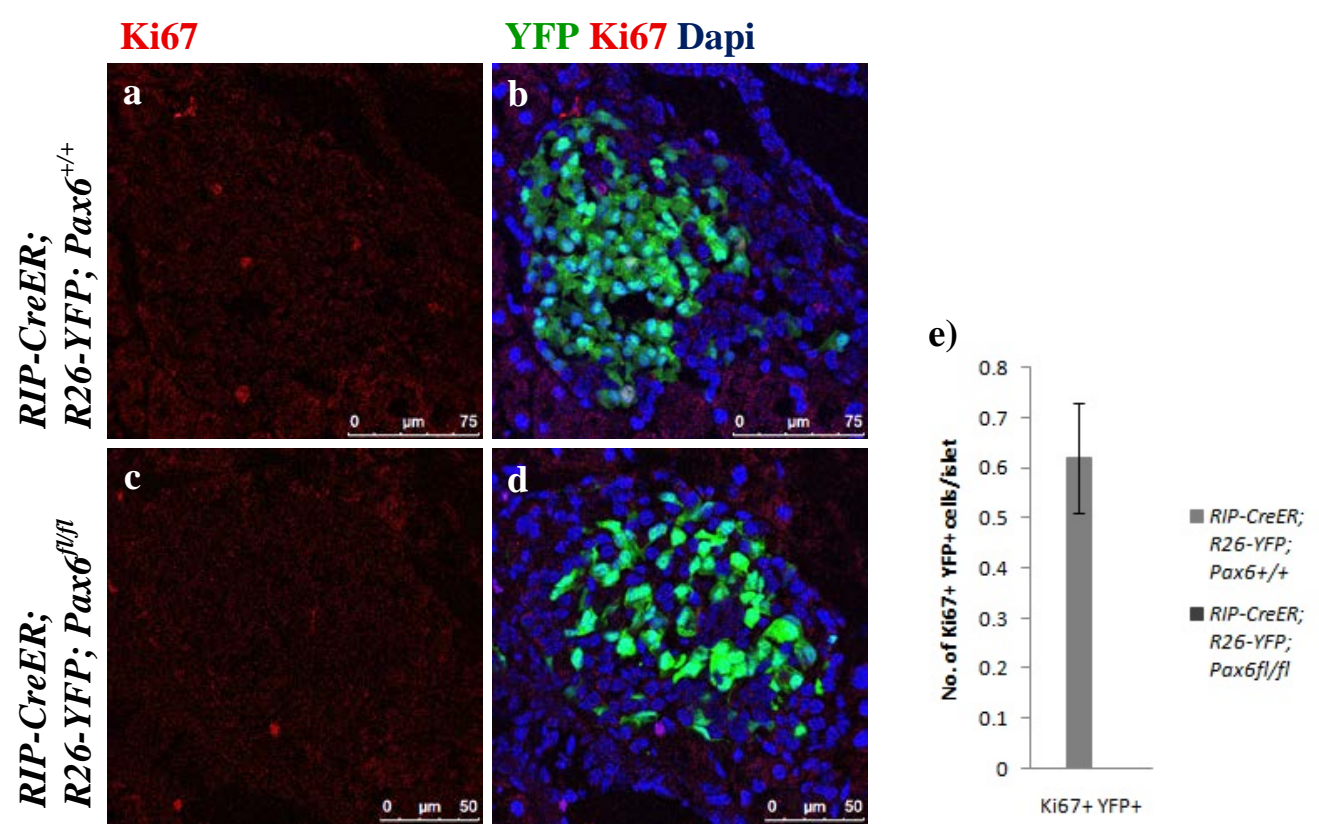

Figure 3.23: Pax6-deficient beta-cells do not proliferate. Immunofluorescence staining of pancreatic cryosections from 7 week old mice at 4 weeks after tamoxifen induction. $\mathrm{Ki}^{+} 7^{+} \mathrm{YFP}^{+}$cells are detected in the control islets (a,b) but not in the beta-cell-specific Pax6 KO islets (c,d). Quantification of $\mathrm{Ki}^{+}{ }^{+} \mathrm{YFP}^{+}$cells in the islets of 7 week old mice at 4 weeks after tamoxifen induction $(n=3)$. Error bars represent SEM. 
binding sites in the promoters of these two genes by using MatInspector (http://www.genomatix.de/). In silico binding sites were found in both promoters. Next we performed ChIP assay to check the actual binding of Pax6 with the identified promoter regions. The ChIP assay performed on Min6 cells did not show any binding of Pax6 with ghrelin or Glut2 promoter (Figure 3.24). Pax6, however, did show binding to the region3 of MafA promoter that was used as a positive control (Raum et al., 2010). Therefore, the upregulation of ghrelin and loss of Glut2 in Pax6 KO islets occurs through some indirect pathway.

\subsubsection{Beta-cell regeneration in the beta-cell-specific Pax6 KO islets}

Regeneration of beta-cells in the diabetic mouse models is an interesting topic of research. As the beta-cell-specific Pax6 KO mice developed hyperglycemia, we were also interested in looking for the possibility of beta-cell regeneration. For this purpose, we injected the mice (containing YFP reporter) with tamoxifen at 3 weeks and 1.5 months of age and analyzed the pancreata at 10 weeks and 4.5 months post tamoxifen induction, respectively. During this time period the non-fasting blood glucose level was also monitored over regular intervals. In both cases the blood glucose level started to rise until it reached a plateau. Afterwards, the blood glucose level slightly decreased in the mice that were injected at 3 weeks of age. However, in the mice that were injected at 1.5 months of age, no decrease was observed in the blood glucose level (Figure 3.25a,b). This showed a slight, but not complete, recovery from hyperglycemia in the mice injected at 3 weeks but not in the mice injected at 1.5 months.

To check for the regeneration of beta-cells at the cellular level, immunofluorescence staining was performed with insulin antibody on the pancreatic sections from these 3 weeks and 1.5 months injected mice. Immediately following the tamoxifen injection nearly $5 \%$ of the insulin ${ }^{+}$cells were YFP $^{-}$, showing the cells that escaped recombination (data not shown). However, long time after the tamoxifen induction an increase in the number of insulin ${ }^{+} \mathrm{YFP}^{-}$cells was observed indicating some regeneration of beta-cells. This increase in the number of insulin ${ }^{+} \mathrm{YFP}^{-}$cells was higher in the mice that were injected at 3 weeks of age as compared to the ones injected at 1.5 months of age (Figure 3.26a,b). This result is also in accordance with the levels of blood glucose. Thus, we conclude that following the ablation of Pax6 some degree of beta-cell regeneration does 
a)

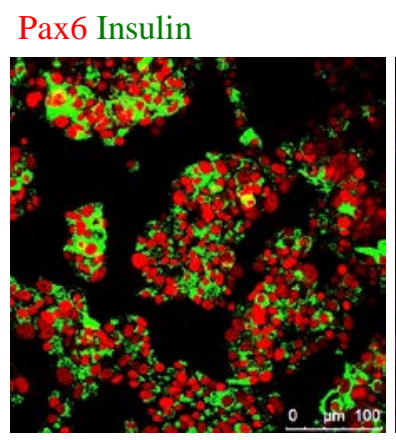

Glut2 Dapi

Pax6 Glut2
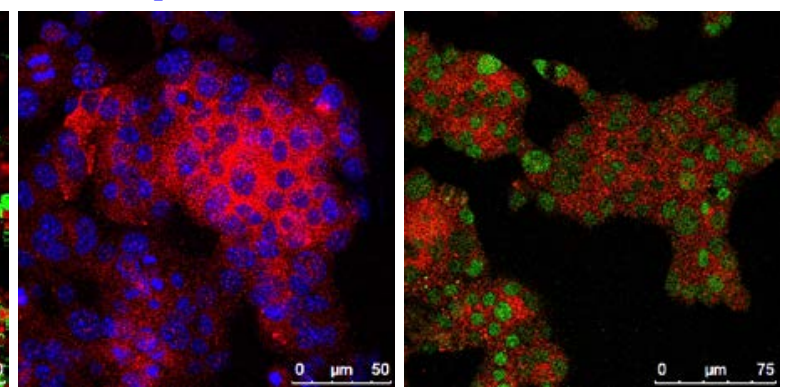

b)

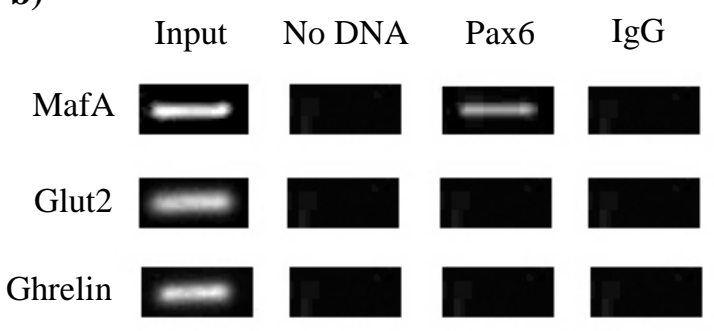

Figure 3.24: Pax6 does not bind to Glut2 and ghrelin promoters. Min6 cells that express Pax6, insulin, and Glut2 were used for ChIP assay (a). Cross-linked chromatin from Min6 cells was precipitated by anti-Pax6 antibody and analyzed by PCR for the corresponding promoter regions (detail in material and methods) (b). Pax6 interacts with MafA promoter region 3 but not with Glut2 and ghrelin promoters. 
a)

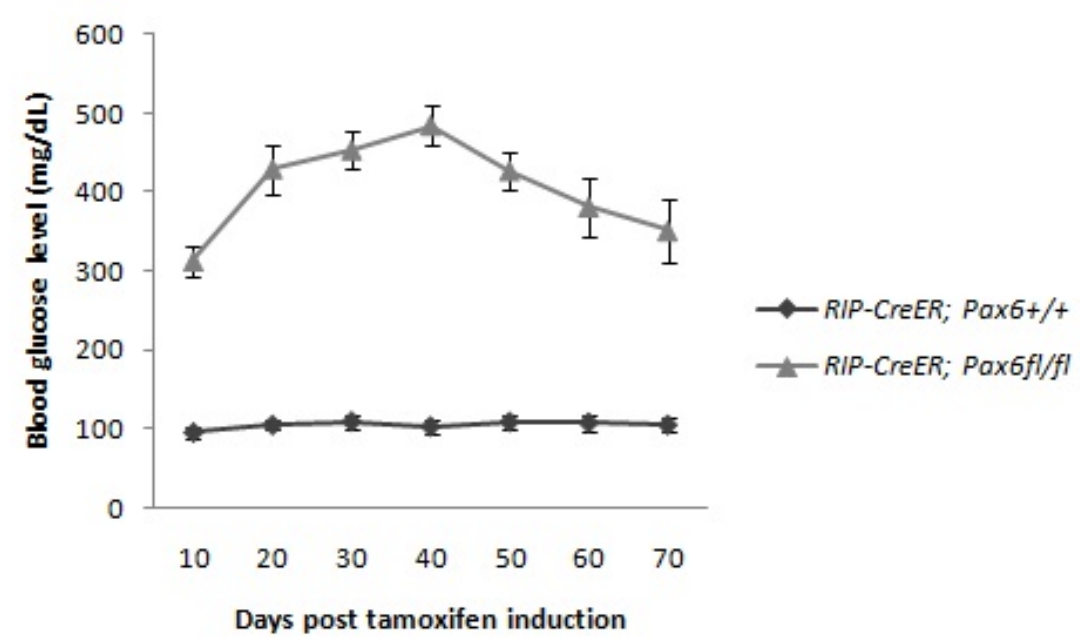

b)

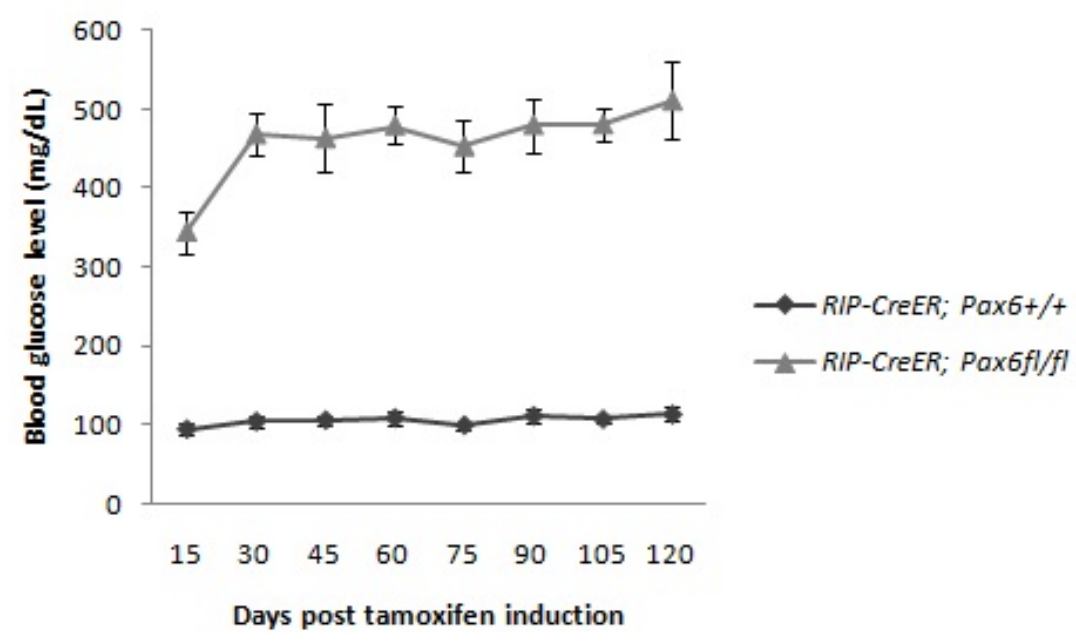

Figure 3.25: Development of diabetes in the beta-cell-specific Pax6 KO mice. Measurement of blood glucose level in mice after tamoxifen induction at 3 weeks (a) or 1.5 month (b) $(n=5)$. Increase in blood glucose level is observed at either age but a slight decrease in the long term is only seen in young mice. Error bars represent SEM. 
a)

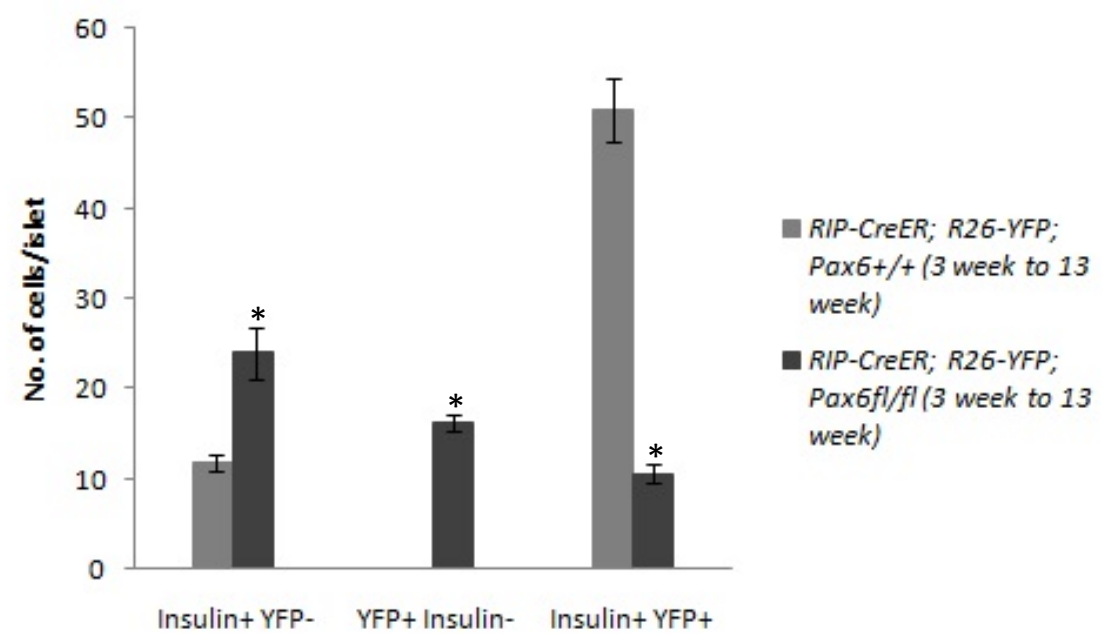

b)

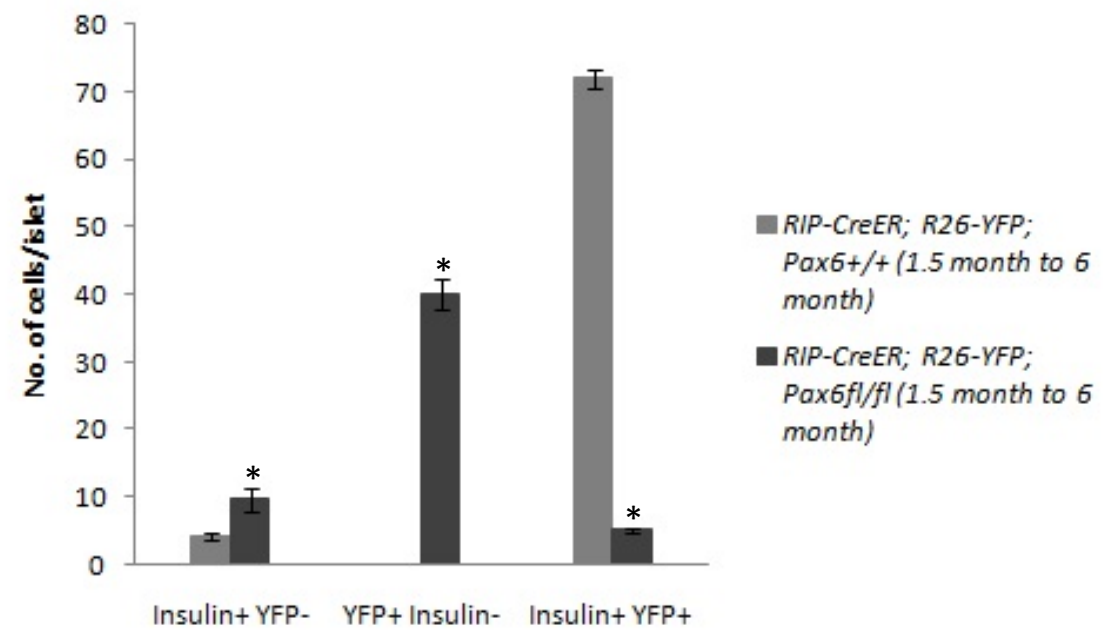

Figure 3.26: Beta-cell regeneration in the beta-cell-specific Pax6 KO mice. Quantification of insulin ${ }^{+} \mathrm{YFP}^{-}, \mathrm{YFP}^{+}$insulin', and insulin ${ }^{+} \mathrm{YFP}^{+}$cells in the islets of 13 week old mice at 10 weeks after tamoxifen induction (a) and 6 month old mice at 4.5 month after tamoxifen induction (b) ( $\mathrm{n}=3)$. Number of insulin ${ }^{+} \mathrm{YFP}^{-}$cells is increased at either age indicating some beta-cell regeneration. Error bars represent SEM; ${ }^{*} \mathrm{p}<0.05$. 
occur and this is more pronounced in the young 3 week old mice compared to the older 1.5 month old mice.

These new beta-cells may arise from proliferation of existing beta-cells that escaped recombination or by transdifferentiation and/or neogenesis (Ahmad, 2013). As compared to the control islets an increased proliferation was not observed in the KO islets. However, compared to the 1.5 month injected mice an increased proliferation was observed in the $\mathrm{YFP}^{-}$insulin $^{+}$cells of the 3 weeks injected mice at 4 weeks post tamoxifen induction (Figure 3.27). Furthermore, in both young (3 week) and older (1.5 month) injected mice $\mathrm{Pdx}^{+}$glucagon ${ }^{+}$cells or $\mathrm{MafB}^{+}$insulin ${ }^{+}$cells were occasionally observed (Figure 3.28). Pdx1 expressing glucagon ${ }^{+}$cells may indicate alpha-cells transdifferentiating to beta-cells (Thorel et al., 2010). On the other hand, MafB expression in some insulin ${ }^{+}$cells can indicate the presence of newly generated insulin ${ }^{+}$cells because MafB is expressed in immature insulin ${ }^{+}$cells during embryonic development (Artner et al., 2007). Therefore, in both young and older Pax6 KO mice new beta-cells may arise from alternative sources.

Regeneration of beta-cells in the beta-cell-specific Pax6 KO mice is, however, not sufficient to allow for the complete recovery from diabetes.

\subsection{Analysis of alpha-cell-specific Pax6 knockout pancreata}

\subsubsection{Conditional knockout of Pax6 in the pancreatic alpha-cells}

In the classical as well as pancreas-specific conditional Pax6 KO mice the number of glucagon positive alpha-cells is drastically reduced (St-Onge et al., 1997; Ashery-Padan et al., 2004). It does show the essential role of Pax6 in the development of alpha-cells. However, as these mice die shortly after birth it does not allow analyzing the role of Pax6 in alpha-cells later in life. Furthermore, if Pax6 is knocked out from the whole islet then the resulting phenotype cannot be specifically linked to the Pax6-deficient alpha-cells. Therefore, to investigate the role of Pax6 in alpha-cell population, we decided to ablate Pax6 from alpha-cells alone. In order to generate the alpha-cell-specific Pax6 KO mice, Pax6 floxed mouse line $\left(\right.$ Pax $\left.^{f l / f l}\right)$ (Ashery-Padan et al., 2000) was crossed with glucagonCre mouse line (Glu-Cre) (Herrera, 2000). Additionally, we incorporated the YFP reporter to identify and trace the Cre-recombined cells (Figure 3.1). This was achieved by including an additional cross with R26-YFP reporter mouse line (Srinivas et al., 2001). 


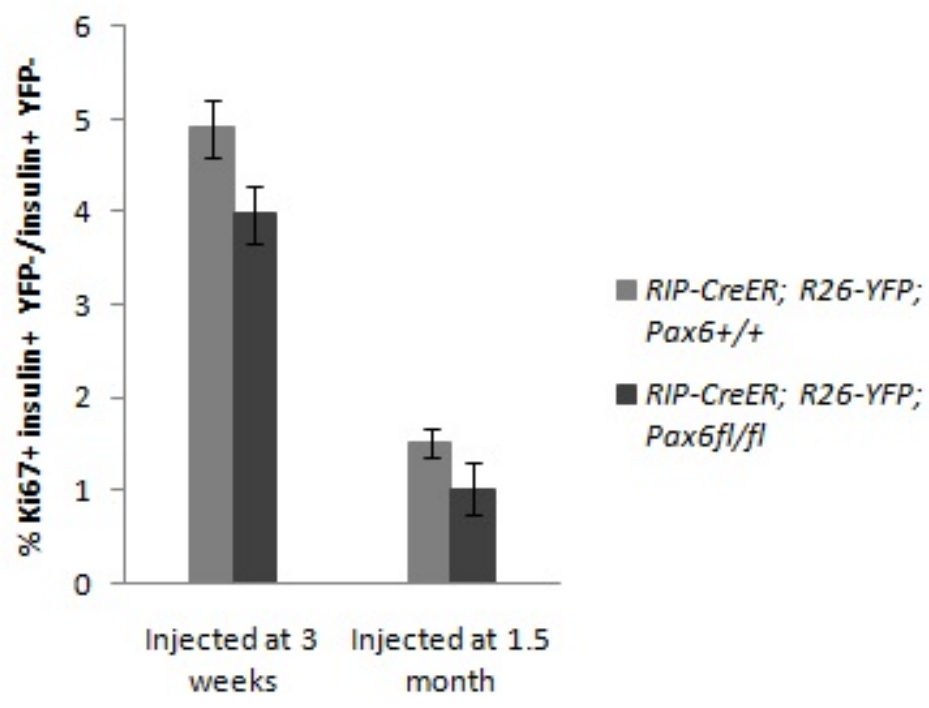

Figure 3.27: Proliferation in the regenerating population of beta-cells in the betacell-specific Pax6 KO islets. Quantification of $\mathrm{Ki}^{+} 7^{+}$insulin ${ }^{+} \mathrm{YFP}^{-}$cells at 4 weeks after tamoxifen induction $(\mathrm{n}=3)$. As compared to the control islets, increased proliferation is not seen in the KO islets. Error bars represent SEM. 

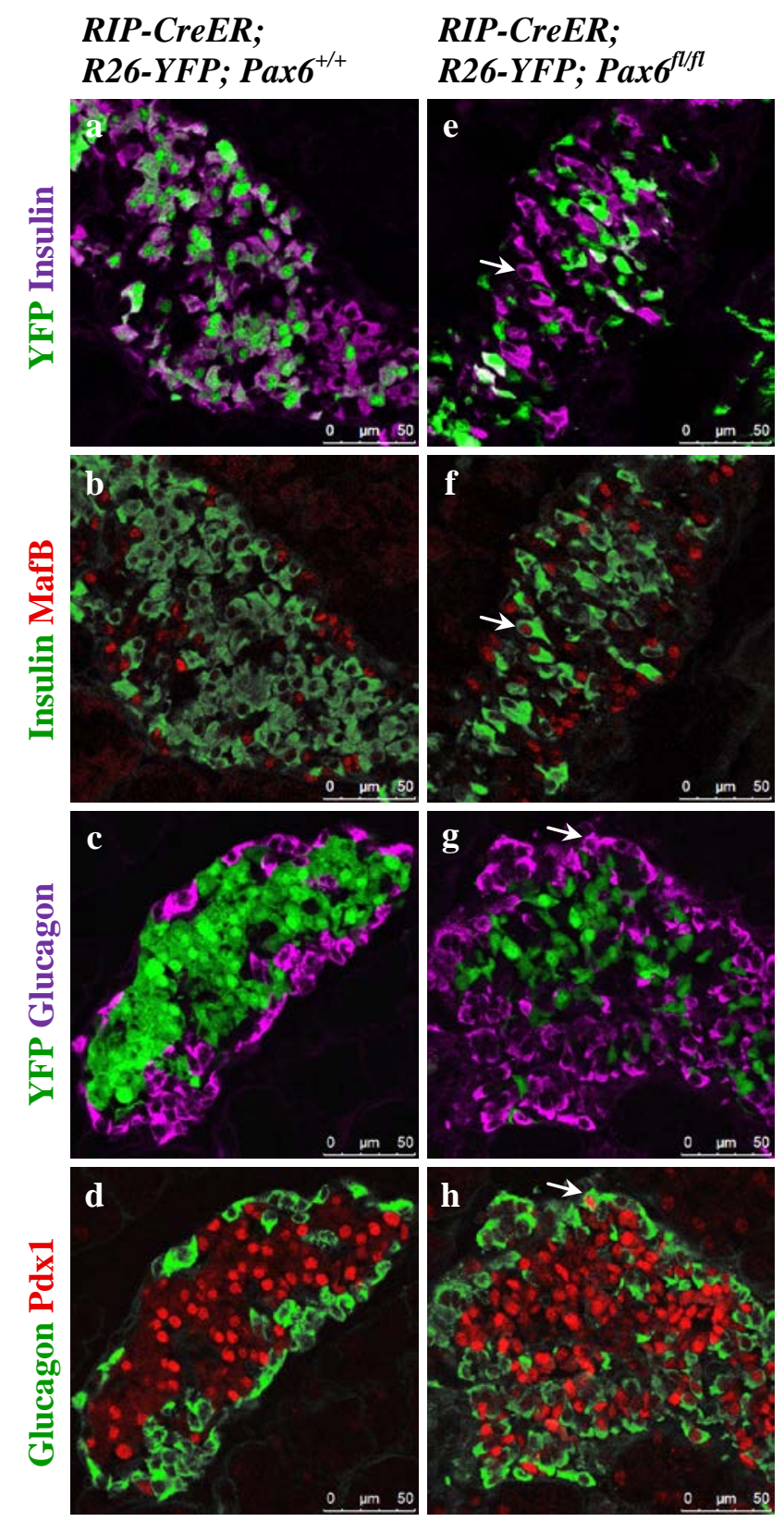

Figure 3.28: Beta-cell regeneration in the beta-cell-specific Pax6 KO islets may occur via transdifferentiation and/or neogenesis. Double immunofluorescence staining of pancreatic cryosections from 2 month old mice at 4 weeks after tamoxifen induction. In the control islets, MafB is not expressed in the insulin ${ }^{+}$cells $(\mathrm{a}, \mathrm{b})$ and $\mathrm{Pdx} 1$ is not expressed in the glucagon ${ }^{+}$cells (c,d). In some of the beta-cell-specific Pax6 KO islets, few insulin ${ }^{+}$cells express MafB (arrow e,f) and few glucagon ${ }^{+}$cells express Pdx1 (arrow g,h) that may indicate neogenesis of beta-cells and/or transdifferentiation of alpha- to beta-cells. 
Successful ablation of Pax6 from the Cre-recombined YFP labeled cells was confirmed by double immunofluorescence staining (Figure 3.29a-f). Quantification of $\mathrm{YFP}^{+}$ glucagon $^{+}$cells showed nearly $70 \%$ recombination efficiency with glucagon-Cre mouse line (Figure 3.29g).

\subsubsection{Ghrelin $^{+}$cells originate from alpha-cells in the alpha-cell-specific Pax6 KO pancreata}

\subsubsection{Co-expression of ghrelin with glucagon in the alpha-cell-specific Pax6 KO pancreata}

Similar to the previous Pax6 KO studies (Kordowich et al., 2011; Hart et al., 2013), we found an increase in the number of ghrelin ${ }^{+}$cells in the alpha-cell-specific Pax6 KO mice. As mentioned before, ghrelin ${ }^{+}$cells are present at a higher number in the embryonic pancreas. At this stage many of the ghrelin ${ }^{+}$cells are co-localized with the glucagon ${ }^{+}$cells (Prado et al., 2004). This makes it difficult to relate the ghrelin upregulation with that of Pax6 ablation. Indeed when we analyzed the control and alpha-cell-specific Pax6 KO pancreata at P0 we found ghrelin-glucagon double positive as well as ghrelin-glucagonYFP triple positive cells in both types of pancreata (Figure 3.30a-h). However, even at this stage the number of ghrelin ${ }^{+}$cells was increased in the KO pancreata (Figure 3.30i). Furthermore, ghrelin ${ }^{+} \mathrm{YFP}^{+}$glucagon ${ }^{-}$cells were only observed in the KO pancreta (Figure 3.30e-h). This indicated the cells that had lost the expression of glucagon as a result of Pax6 ablation. Loss of glucagon expression in Pax6-deficient alpha-cells was expected as Pax6 has been shown to control the transcription of glucagon gene (Sander et al., 1997; Gosmain et al., 2010).

As compared to the embryonic or early postnatal pancreas, in the adult pancreas the expression of ghrelin decreases to undetectable level (Wierup et al., 2004). That is why the ghrelin positive cells are very rare to observe in the adult pancreas from control mice. Accordingly, we detected no ghrelin expression in the adult control islets. In comparison to that the number of ghrelin ${ }^{+}$cells was increased in the alpha-cell-specific Pax6 KO islets and nearly all of these cells were co-localized with the YFP labeled cells confirming their origin from Cre-recombined cells (Figure 3.31 and 3.32). Additionally, we observed that majority of these ghrelin ${ }^{+} \mathrm{YFP}^{+}$cells in the $\mathrm{KO}$ islets were negative for the glucagon 


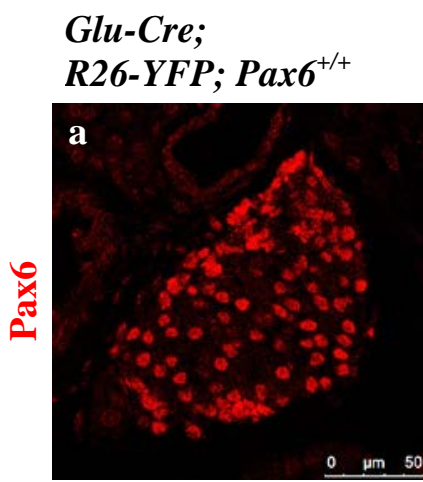

\section{Glu-Cre;}

R26-YFP; Pax $^{f l / f l}$
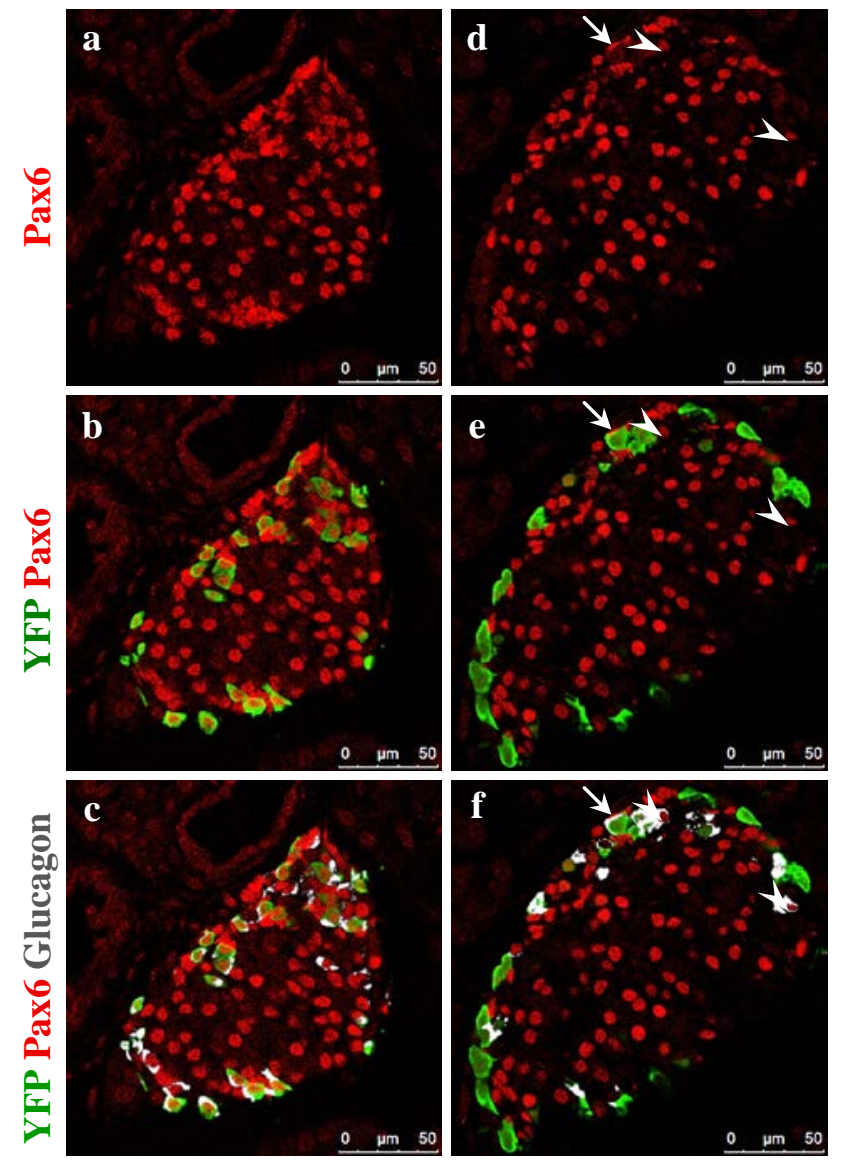

g)

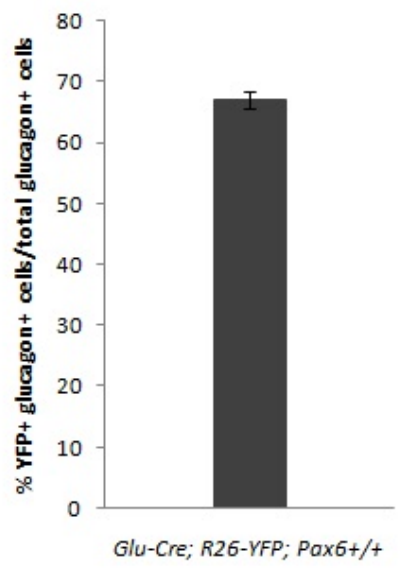

Figure 3.29: Alpha-cell-specific ablation of Pax6. Double immunofluorescence staining of pancreatic cryosections from 1 month old mice. In the control islets, all the $\mathrm{YFP}^{+}$cells express Pax6 and glucagon (a-c). In the alpha-cell-specific Pax6 KO islets, most of the $\mathrm{YFP}^{+}$cells are negative for Pax6 and have lost the expression of glucagon as well (d-f). YFP $^{-}$glucagon $^{+}$cells in the $\mathrm{KO}$ islets express Pax6 (arrowheads d-f). Rarely $\mathrm{YFP}^{+}$ glucagon $^{+}$Pax6 $^{-}$cells are also found in the KO islets (arrows d-f). Quantification of $\mathrm{YFP}^{+}$ glucagon $^{+}$cells in relation to the total glucagon ${ }^{+}$cells in 1 month old mice $(g)(n=3)$. Nearly $70 \%$ of the glucagon ${ }^{+}$cells are labeled with YFP. Error bars represent SEM. 


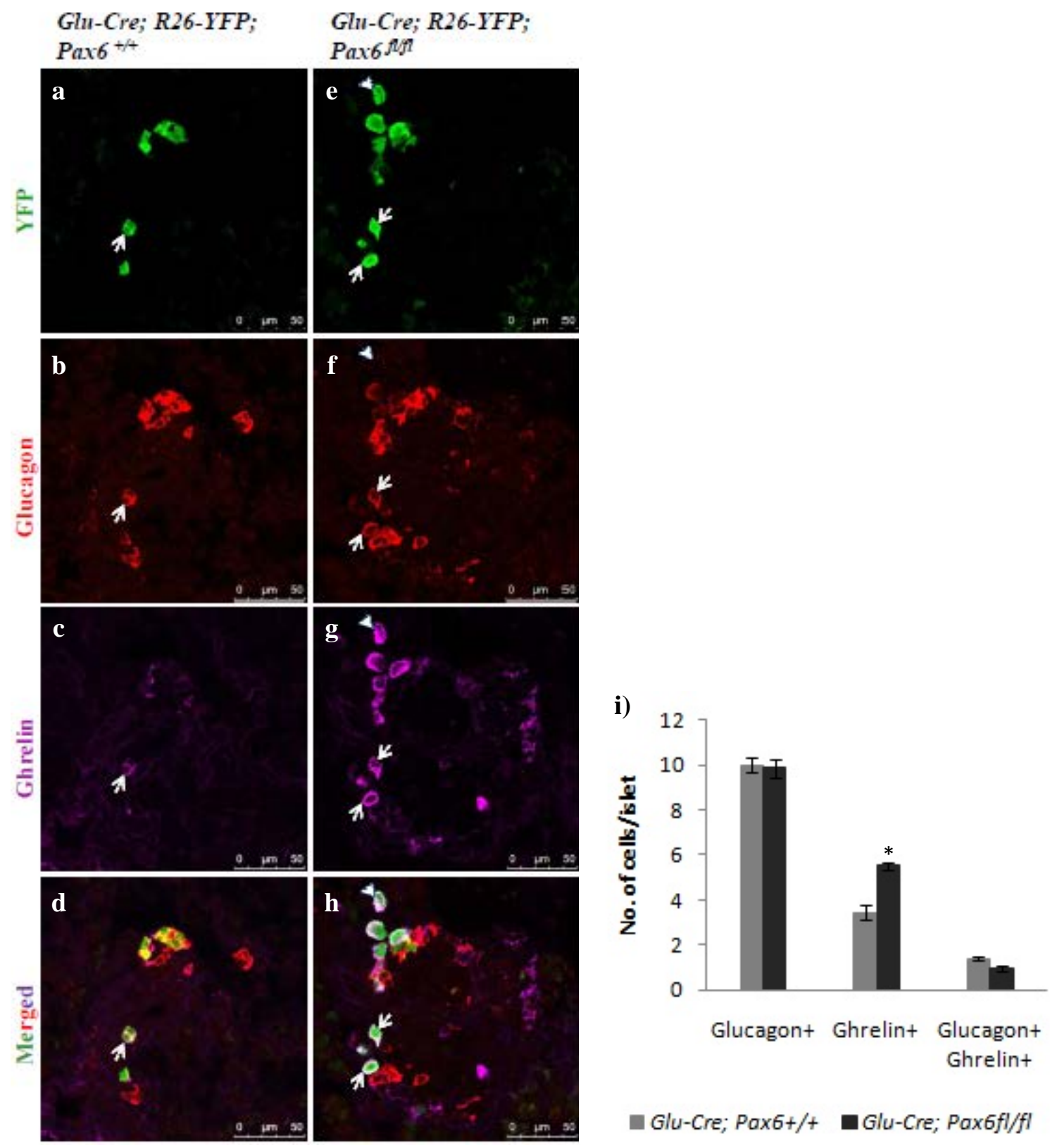

Figure 3.30: A mixed population of ghrelin and glucagon positive cells at P0. Double immunofluorescence staining of pancreatic cryosections at P0. In both the control and alpha-cell-specific Pax6 KO islets, YFP-glucagon-ghrelin triple positive cells are detectable (arrows a-h). Additionally, in the alpha-cell-specific Pax6 KO islets, YFP ${ }^{+}$ ghrelin $^{+}$glucagon ${ }^{-}$cells are deteced (arrowhead e-h) that are not found in the control islets. Quantification of glucagon ${ }^{+}$, ghrelin ${ }^{+}$, and glucagon-ghrelin co-positive cell population at P0 (i) (n=3). Ghrelin ${ }^{+}$cells are increased in the KO pancreata while glucagon $^{+}$cell population is not significantly changed. Error bars represent SEM; ${ }^{*} \mathrm{p}<0.05$. 


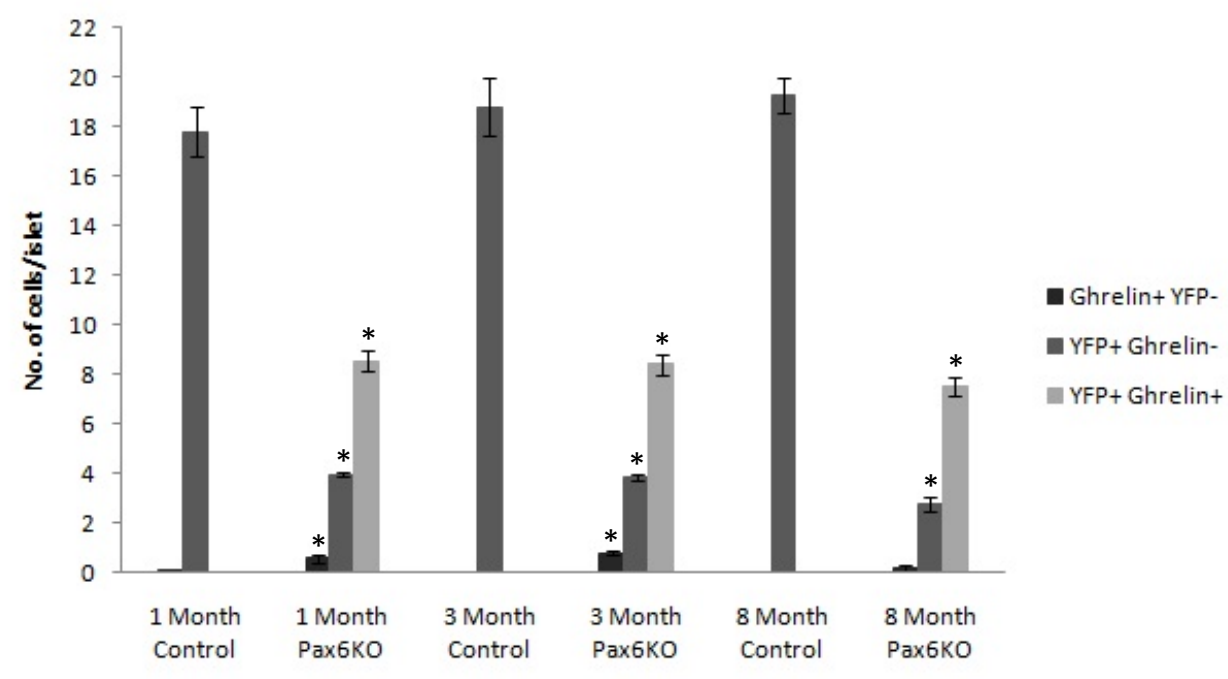

Figure 3.31: Ghrelin $^{+}$cell population is increased in the adult alpha-cell-specific Pax6 KO islets. Quantification of ghrelin ${ }^{+} \mathrm{YFP}^{-}, \mathrm{YFP}^{+}$ghrelin', and $\mathrm{YFP}^{+}$ghrelin $^{+}$cells at 1 month, 3 months, and 8 months of age (c) $(n=3)$. As compared to the control islets, number of $\mathrm{YFP}^{+}$ghrelin ${ }^{+}$cells is significantly increased in the $\mathrm{KO}$ islets at all the ages analyzed. However, $\mathrm{YFP}^{+}$ghrelin $^{+}$cell population is not significantly changed within the KO group at different ages. Error bars represent SEM; *p<0.05. 


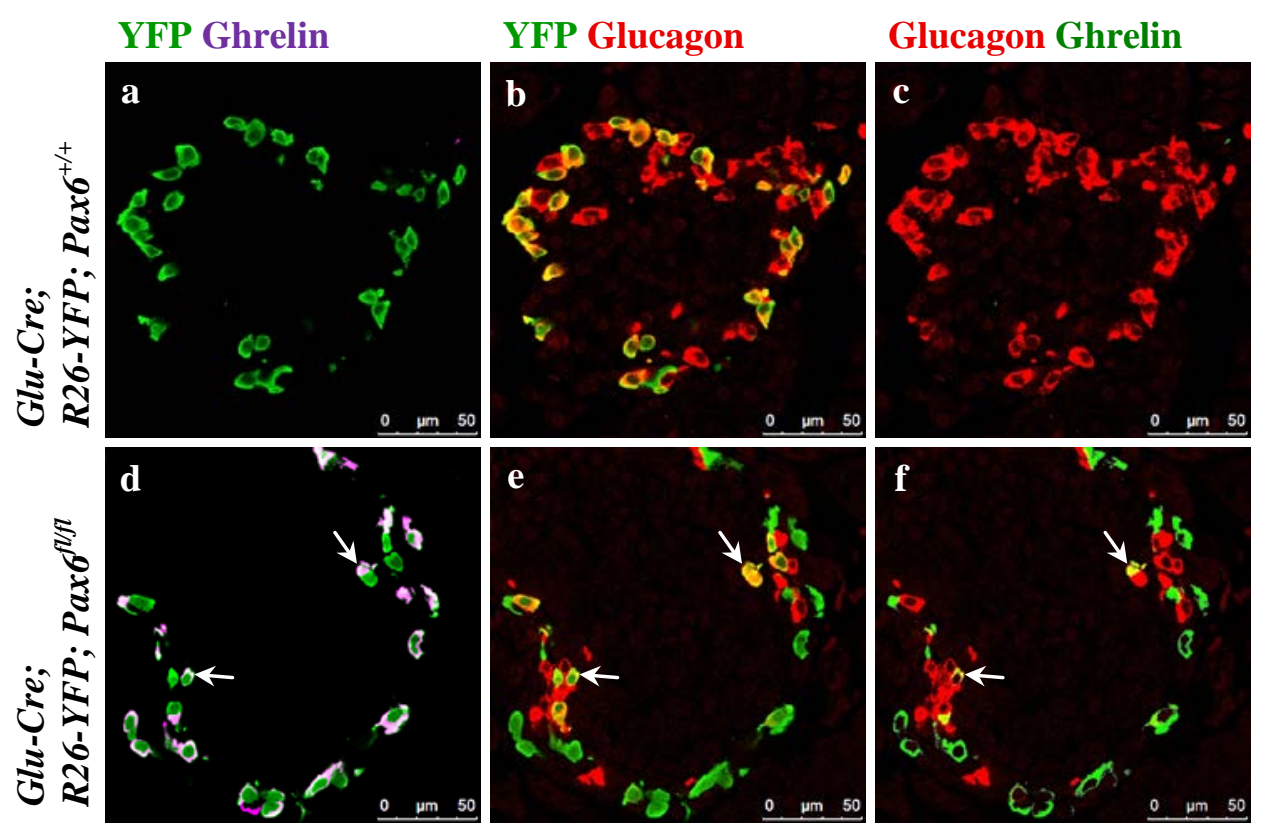

Figure 3.32: Ghrelin ${ }^{+}$cells originate from alpha-cells in the alpha-cell-specific Pax6 KO islets. Double immunofluorescence staining of pancreatic cryosections from 1 month old mice. Ghrelin expression is not detected in the control islets (a-c). In the alpha-cellspecific Pax6 KO islets, ghrelin expression is upregulated in YFP labeled cells (d-f) and rarely ghrelin expression co-localizes with glucagon expression (arrows d-f). 
expression but it was possible to observe some ghrelin-glucagon-YFP triple positive cells in the KO islets (Figure 3.32). In the adult islet these cells may indicate an intermediate state of conversion from glucagon to ghrelin expressing cells. Thus, we conclude that alpha-cells lose the expression of glucagon and start to express ghrelin following the ablation of Pax6.

\subsubsection{Expression of alpha-cell related transcription factors in the ghrelin ${ }^{+}$cell population of alpha-cell-specific Pax6 KO islets}

In the next step we checked the expression of alpha-cell related transcription factors MafB and Arx in the YFP labeled Pax6-deficient alpha-cells that now expressed ghrelin instead of glucagon. As compared to the YFP labeled cells in the control islets, YFP labeled ghrelin ${ }^{+}$cells in the KO islets were negative for MafB but they still expressed Arx. Additionally, in some KO islets few $\mathrm{YFP}^{-}$ghrelin ${ }^{+}$cells were detected that were also negative for MafB and positive for Arx (Figure 3.33 and 3.34). MafB is specifically expressed in adult alpha-cells and is important for the glucagon gene transcription (Artner et al., 2006). On the other hand, expression of Arx defines the alpha-cell lineage (Collombat et al., 2003, 2005, 2007). Therefore, absence of MafB and presence Arx shows the loss of mature alpha-cell function in the Pax6-deficient alpha-cells but preservation of the alpha-cell lineage. Furthermore, persistent expression of Arx in YFP labeled ghrelin ${ }^{+}$cells is another proof of their origin from alpha-cells.

\subsubsection{Regeneration of alpha-cells in the alpha-cell-specific Pax6 KO islets}

Alpha-cell regeneration has been shown in some models of glucagon deficiency (Furuta et al., 1997; Gelling et al., 2003). As Pax6 KO also led to the loss of glucagon expression, we were interested in looking for the regeneration of alpha-cells. The number of total glucagon $^{+}$cells in the $\mathrm{KO}$ islets was not significantly different from that in the control islets at either 1 month or 3 months of age but was significantly reduced at 8 months of age (Figure 3.35a). This means that at younger age some alpha-cell regeneration was taking place. To clarify this further, we quantified the number of $\mathrm{YFP}^{-}$glucagon $^{+}$and $\mathrm{YFP}^{+}$glucagon ${ }^{+}$cells in control and $\mathrm{KO}$ islets. We found that the number of $\mathrm{YFP}^{-}$ glucagon $^{+}$cells was significantly increased in the KO islets at 1 month and 3 months of age but not at 8 months of age (Figure 3.35b). 

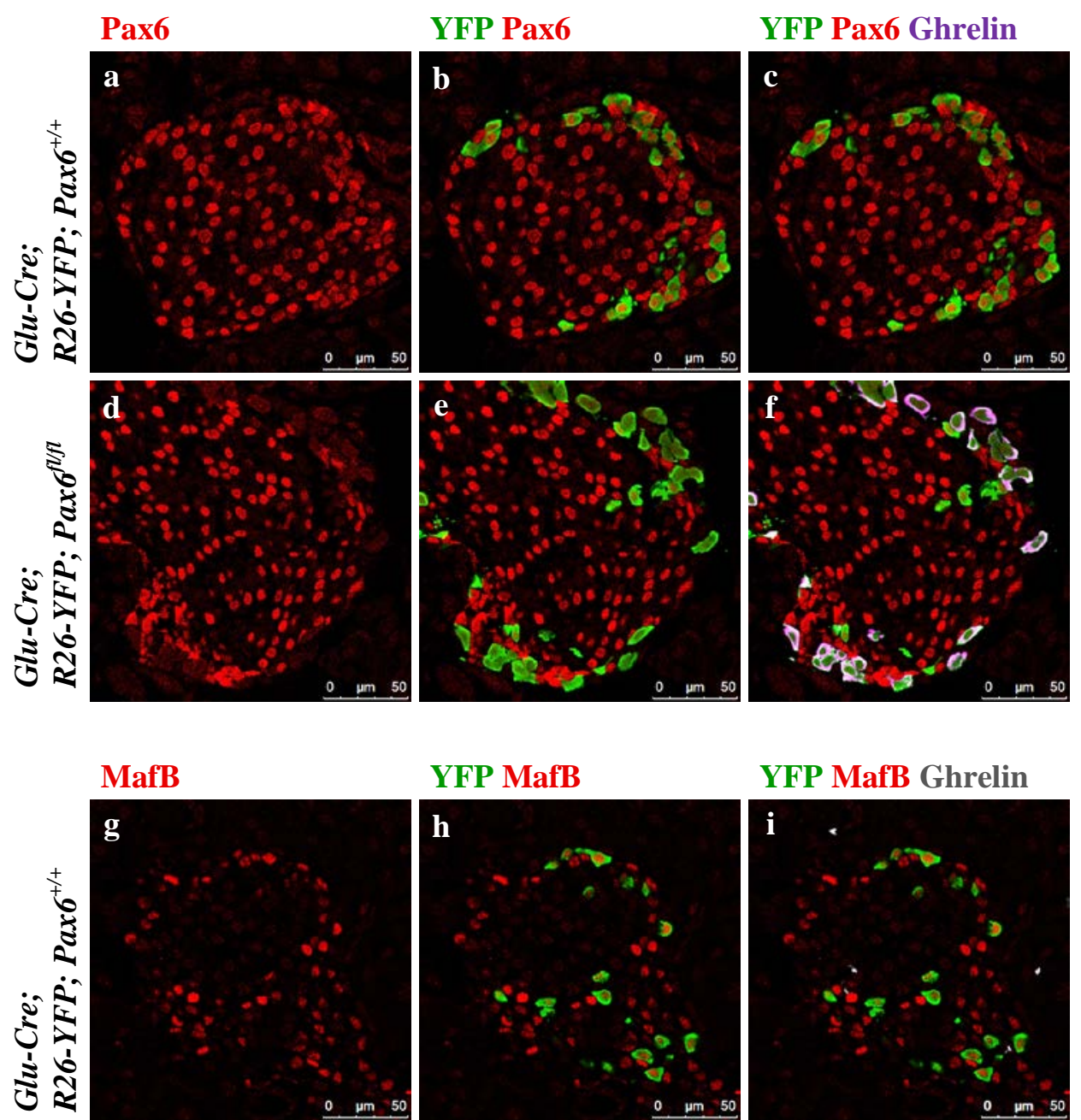

YFP MafB Ghrelin
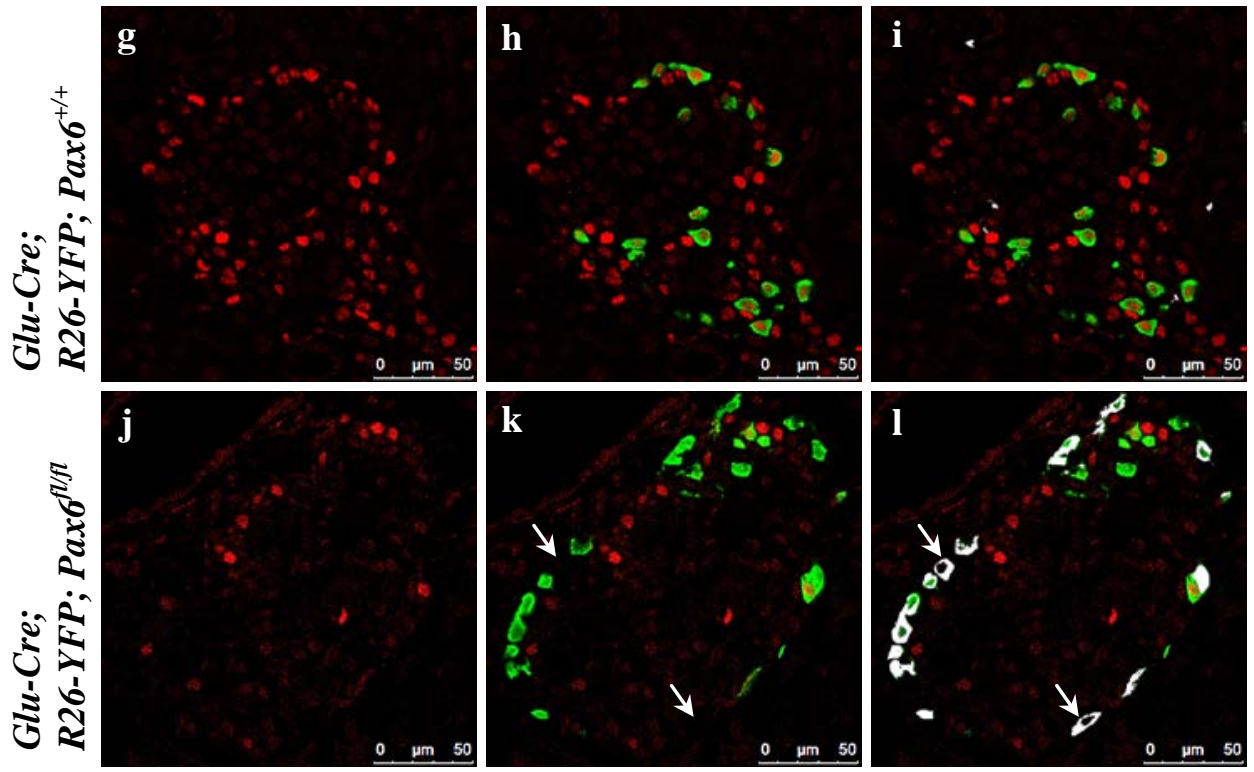

Figure 3.33: Expression of alpha-cell related transcription factors in the ghrelin ${ }^{+}$ cells of the alpha-cell-specific Pax6 KO islets. Double immunofluorescence staining of pancreatic cryosections from 1 month old mice. Ghrelin expression is not detected in the control islets (a-c and g-i). In the alpha-cell-specific Pax6 KO islets, ghrelin expression is upregulated in YFP labeled cells and these ghrelin ${ }^{+}$cells are negative for Pax6 (d-f) and MafB (j-l) expression. Rarely some $\mathrm{YFP}^{-}$ghrelin $^{+}$cells are also detected in the KO islets and they are also negative for MafB expression (arrows k,l). 


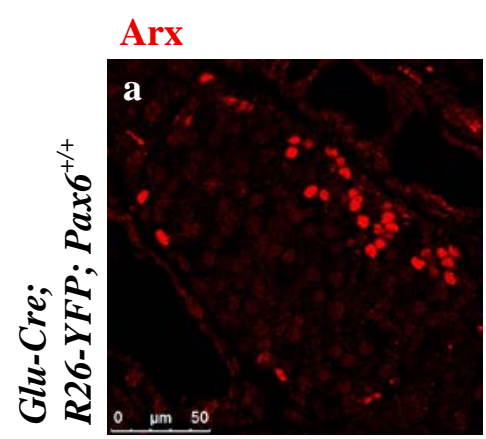

Arx Ghrelin
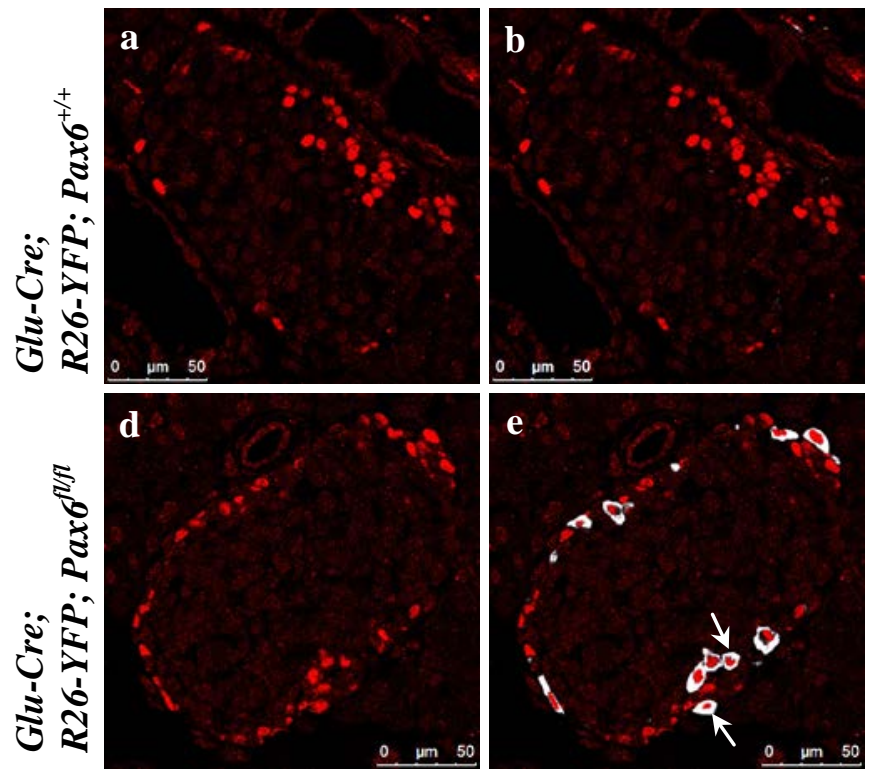

YFP Arx Ghrelin
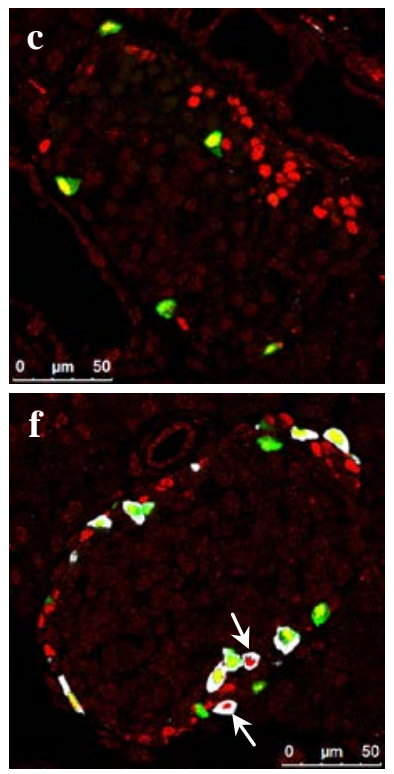

Figure 3.34: Expression of alpha-cell-lineage determinant Arx in the ghrelin ${ }^{+}$cells of the alpha-cell-specific Pax6 KO islets. Double immunofluorescence staining of pancreatic cryosections from 1 month old mice. Ghrelin expression is not detected in the control islets (a-c). In the alpha-cell-specific Pax6 KO islets, ghrelin expression is upregulated in YFP labeled Pax6-deficient cells and these ghrelin ${ }^{+}$cells are positive for Arx expression (d-f). Rarely some $\mathrm{YFP}^{-}$ghrelin $^{+}$cells are also detected in the KO islets and they are also positive for Arx expression (arrows e,f). 
a)

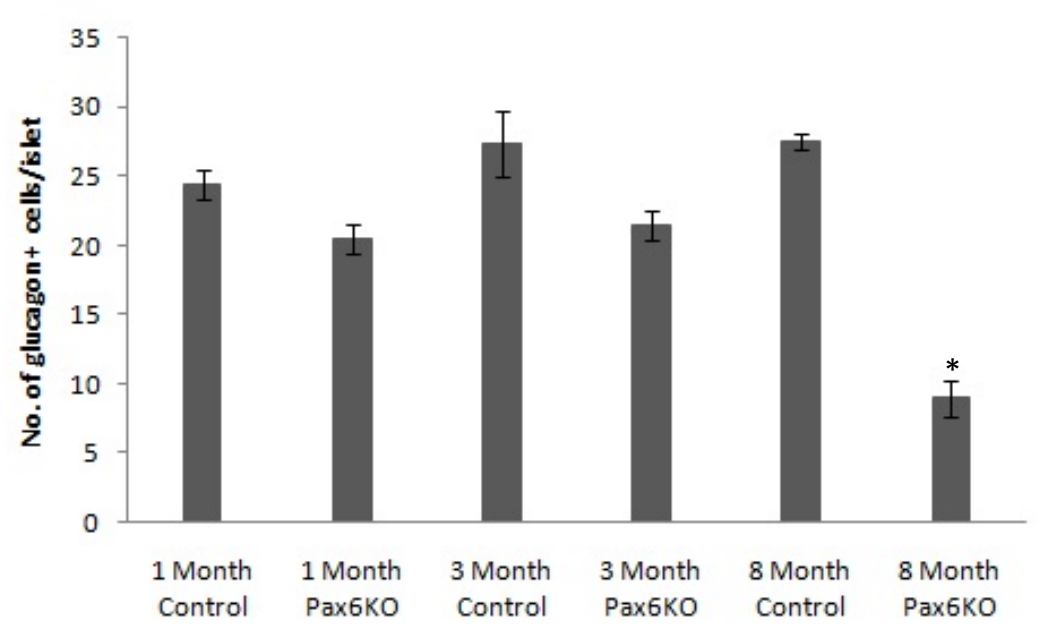

b)

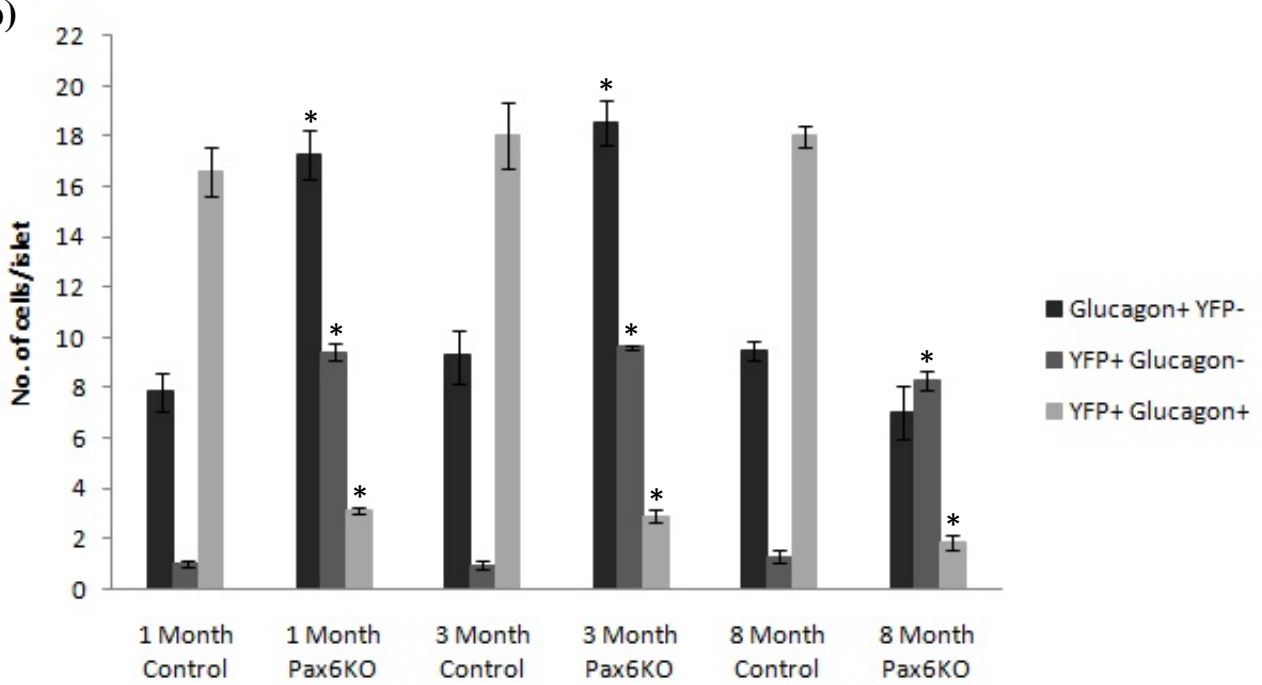

Figure 3.35: Alpha-cell regeneration in the alpha-cell-specific Pax6 KO islets.

Quantification of total glucagon ${ }^{+}$cell pouplation at 1 month, 3 months, and 8 months of age (a) $(\mathrm{n}=3)$. Number of total glucagon ${ }^{+}$cells is significantly reduced in the $\mathrm{KO}$ islets at 8 months of age but not at 1 month or 3 months of age. Quantification of glucagon ${ }^{+} \mathrm{YFP}^{-}$, $\mathrm{YFP}^{+}$glucagon ${ }^{-}$, and $\mathrm{YFP}^{+}$glucagon ${ }^{+}$cells at 1 month, 3 months, and 8 months of age (b) $(n=3)$. Number of glucagon ${ }^{+}$YFP $^{-}$cells is significantly increased in the KO islets at 1 month and 3 months of age but not at 8 months of age. Error bars represent SEM; ${ }^{*} \mathrm{p}<0.05$. 
This indicated the regeneration capacity of alpha-cells at young and middle age but a loss of regeneration at older age. However, even the older mice did not show any symptoms of glucagon deficiency because nearly 30\% population of alpha-cells that escaped recombination was still present.

\subsubsection{B2 is upregulated and PC2 not affected in the alpha-cell-specific Pax6 KO islets}

Proglucagon, the precursor of glucagon, is processed in alpha-cells by prohormone convertase 2 (PC2) to generate the mature glucagon peptide. The neuropeptide 7B2 is involved in the activation of PC2 (Fortenberry et al., 2002; Katz et al., 2009). To see how proglucagon processing is affected in Pax6-deficient alpha-cells, we checked the expression of PC2 and 7B2. We found that in comparison to the control islets the expression of PC2 was not affected and that of 7B2 highly upregulated in the YFP labeled Pax6-deficient alpha-cells (Figure 3.36). Therefore, a loss of proglucagon processing via PC2 downregulation does not seem to occur in these cells.

\subsection{Pax6 overexpression}

\subsubsection{Generation of transgenic mice conditionally overexpressing Pax6}

For conditional overexpression of Pax6, Jojo vector was used (Collombat et al., 2007). Pax6 cDNA was cloned into this vector to generate the overexpression construct. The construct contained an EGFP (enhanced green fluorescent protein) cassette flanked by loxP sites under the control of cytomegalovirus (CMV) early enhancer/beta-actin (CAG) promoter that provides high level of constitutive expression. The EGFP cassette was followed by Pax6 cDNA, an internal ribosome entry site (IRES), and beta-galactosidase encoding sequence (Figure 3.37a). In the absence of Cre-mediated recombination only EGFP is expressed from this construct because of the transcriptional stop site present after EGFP. However, in the presence of Cre, recombination of loxP sites removes the EGFP cassette along with the transcriptional stop and allows for the expression of Pax6 along with beta-galactosidase in the Cre expressing cells (Figure 3.37b). Expression of beta-galactosidase, therefore, serves as a marker of overexpression. 


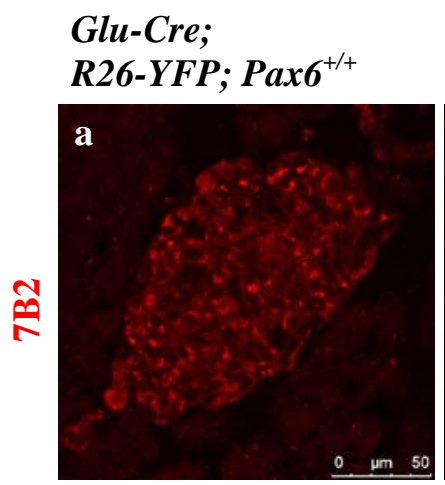

Glu-Cre; R26-YFP; Pax6 $6^{f l / l}$
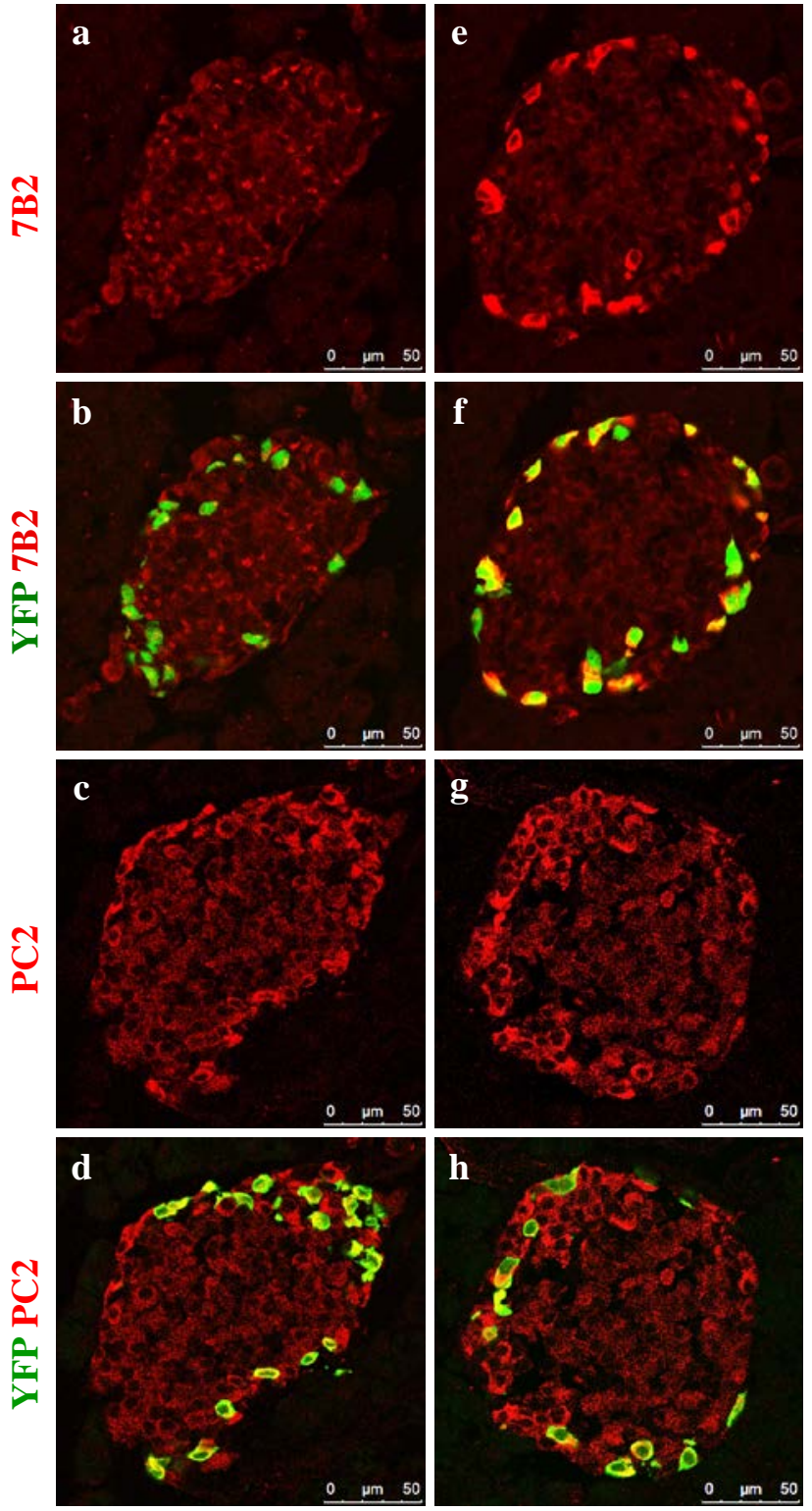

Figure 3.36: Expression of $7 \mathrm{~B} 2$ and PC2 in the alpha-cell-specific Pax6 KO islets. Immunofluorescence staining of pancreatic cryosections from 1 month old mice. 7B2 expression is very low in the control islets (a,b). In the alpha-cell-specific Pax6 KO islets, 7B2 expression is higly upregulated in YFP labeled Pax6-deficient cells (e,f). Compared to the control islets, PC2 expression is unchanged in the alpha-cell-specific Pax6 KO islets (c,d and g,h). 


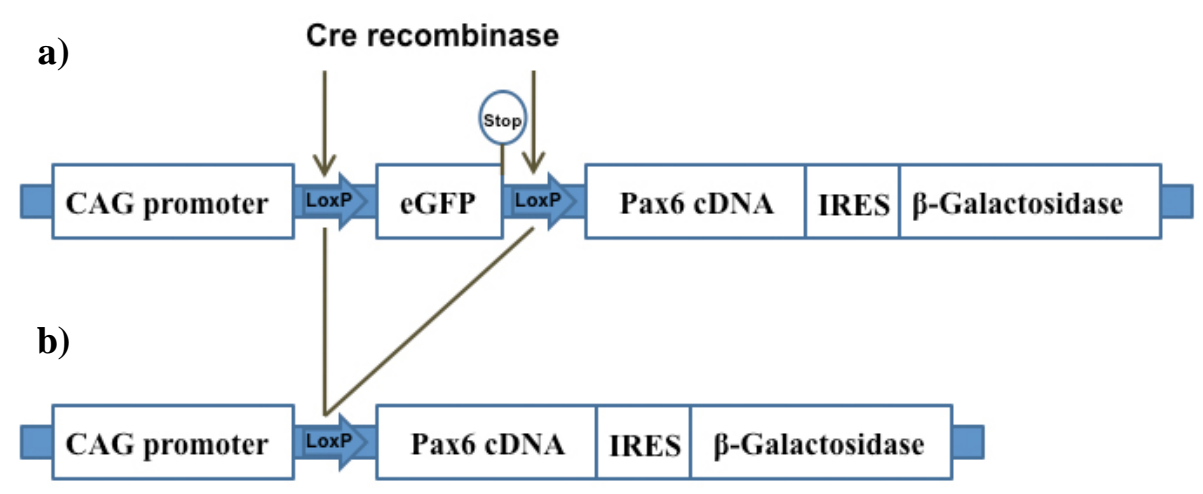

Figure 3.37: Construct used to generate conditional Pax6-overexpressing (Pax6OE) transgenic mice. (a) Before Cre-mediated recombination, only GFP is expressed due to the transcriptional stop present after it. (b) After Cre-mediated recombination, GFP cassette (together with the stop) is removed allowing the expression of Pax6 and betagalactosidase in the Cre expressing cells. 
This construct was then used for the pronuclear injection to generate three GFP expressing transgenic mouse lines (termed Pax6OE), all of which gave germ-line transmission. Line 3 and 2 were used for all of the experiments. These Pax6OE mice were then crossed with different Cre mouse lines including Pdx1-Cre (for overexpression in the whole pancreas), insulin-Cre (Ins-Cre, for overexpression in beta-cells), and glucagon-Cre (Glu-Cre, for overexpression in alpha-cells) (Gannon et al., 2000; Herrera, 2000).

In the Pax6OE; Pdx1-Cre pancreata GFP expression was lost from most of the pancreatic tissue and Pax6 expression was found in the whole pancreas including the exocrine tissue where Pax6 is normally not expressed (Figure 3.38a-f). This indicated the correct functioning of the Pax6OE construct. In case of Pax6OE; Ins-Cre and Pax6OE; Glu-Cre mice the overexpression of Pax6 was confirmed by beta-galactosidase staining and X-Gal staining, respectively (Figure 3.38g-j). Furthermore, overexpression of Pax6 was also confirmed by qRT-PCR in Pax6OE; Pdx1-Cre and Pax6OE; Ins-Cre mice (Figure 3.38k).

\subsubsection{Pancreatic phenotype of Pax6 overexpressing mice}

There was no difference in the blood glucose level of Pax6OE; Pdx1-Cre, Pax6OE; InsCre, and Pax6OE; Glu-Cre mice as compared to the Pax6OE control mice. At the pancreatic level, we observed slight hypoplasia of the pancreatic tissue in the Pax6OE; Pdx1-Cre mice as compared to the Pax6OE control mice (Figure 3.39). In the endocrine pancreas, islet structure was intact and the number of insulin ${ }^{+}$and somatostatin ${ }^{+}$cells was not significantly different in the Pax6OE; Pdx1-Cre mice as compared to the Pax6OE control mice at 3 weeks of age (data not shown). However, the number of glucagon ${ }^{+}$and $\mathrm{PP}^{+}$cells was significantly reduced in Pax6OE; Pdx1-Cre mice as compared to the Pax6OE control mice at 3 weeks of age (Figure 3.40a). Expression of various beta-cell related factors was also not changed in the Pax6OE; Pdx1-Cre mice as compared to the Pax6OE control mice (Figure 3.41).

We found in our study that ghrelin expression upregulates in both the alpha- and betacell-specific Pax6 KO pancreata. In relation to that we were interested to see the effect of Pax6 overexpression on ghrelin ${ }^{+}$cell population. In the adult islet, ghrelin ${ }^{+}$cell 


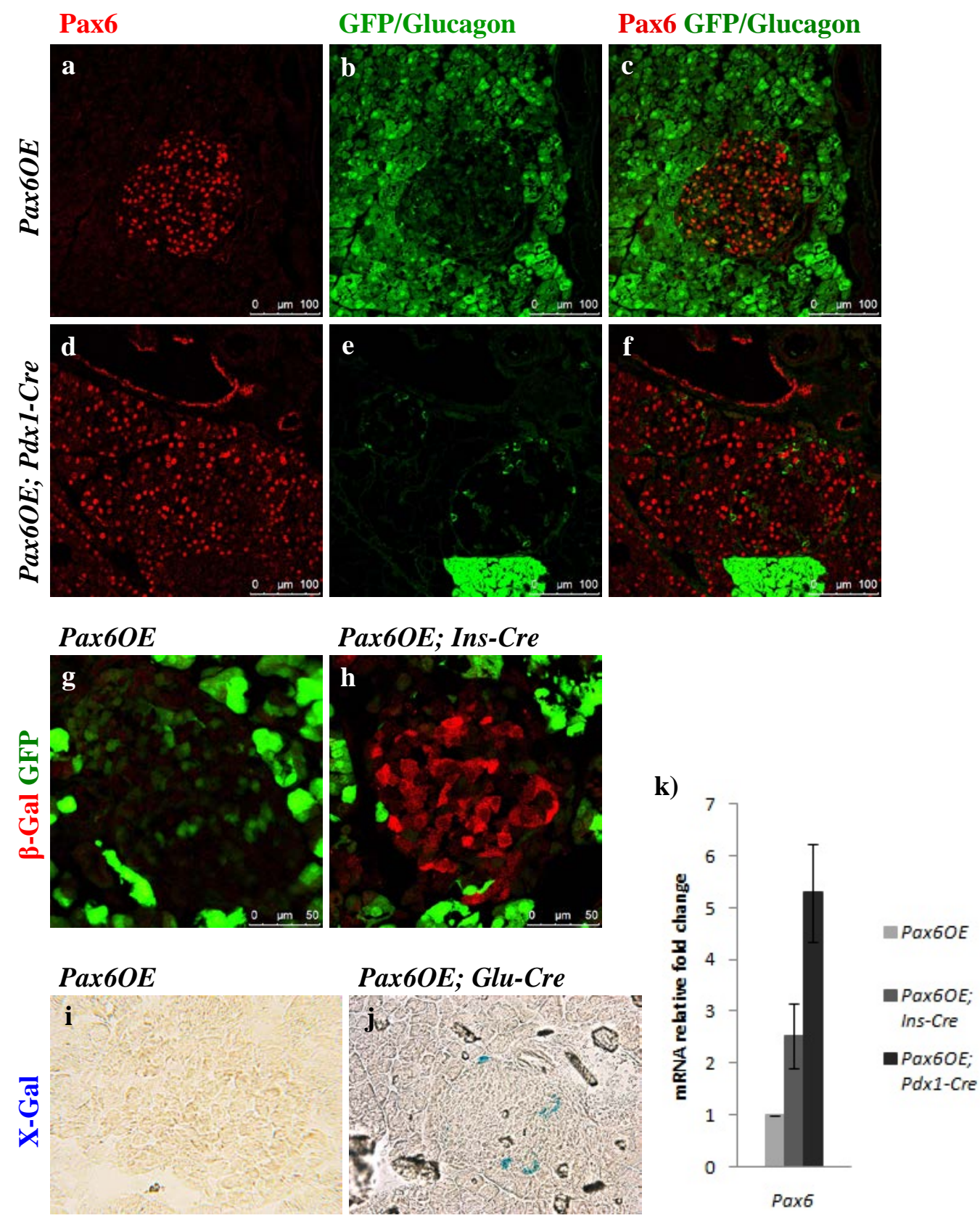

Figure 3.38: Pax6 overexpression under Pdx1, insulin, and glucagon expression domains. Immunofluorescence staining $(\mathrm{a}-\mathrm{h})$ and $\mathrm{X}-\mathrm{Gal}$ staining $(\mathrm{i}, \mathrm{j})$ of pancreatic cryosections from 2 month old mice. In Pax6OE control pancreata, Pax6 is expressed only inside the endocrine cells of the islets (a-c). In Pax6OE; Pdx1-Cre pancreata, Pax6 is expressed in the whole pancreas including both the endocrine and exocrine tissue (d-f). Pax6 overexpression indicated by the beta-galactosidase expression in Pax6OE; Ins-Cre islets (h), and by the X-Gal staining in Pax6OE; Glu-Cre islets (j). Quantitative RT-PCR of Pax6 mRNA in the pancreata of 2 month old mice shows overexpression in Pax6OE; Pdx1-Cre and Pax6OE; Ins-Cre mice (k) (n=3). Error bars represent SEM. 


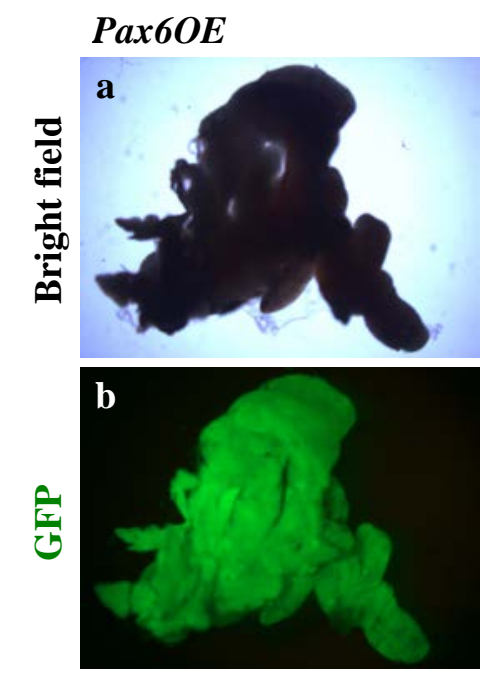

Pax6OE; Pdx1-Cre

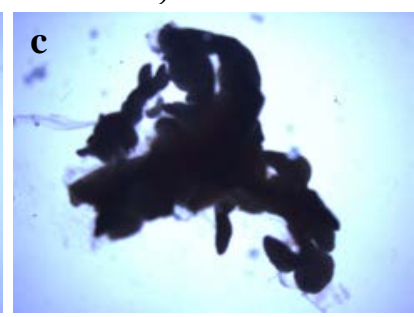

d

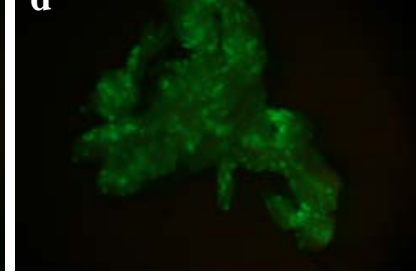

Figure 3.39: Pancreatic hypoplasia in Pax6OE; Pdx1-Cre mice. Pancreatic tissue is slightly reduced in size in Pax6OE; Pdx1-Cre mice (c,d) compared to the Pax6OE control mice (a,b) at 4 months of age. Absence of GFP expression in (d) shows the successful Cre-mediated recombination.

a)

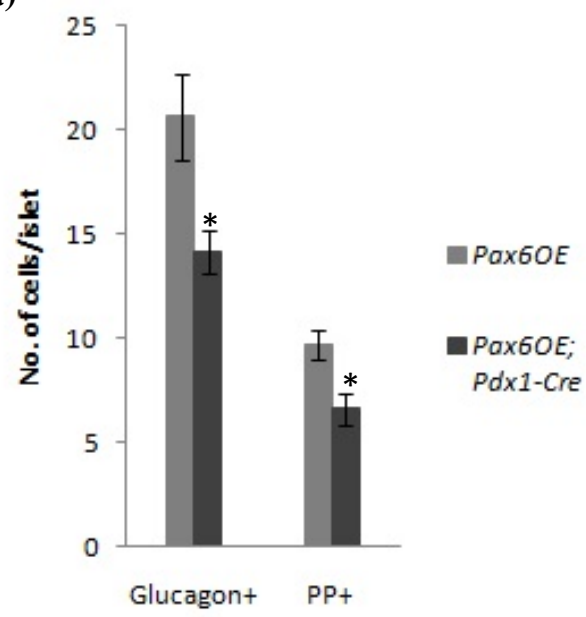

b)

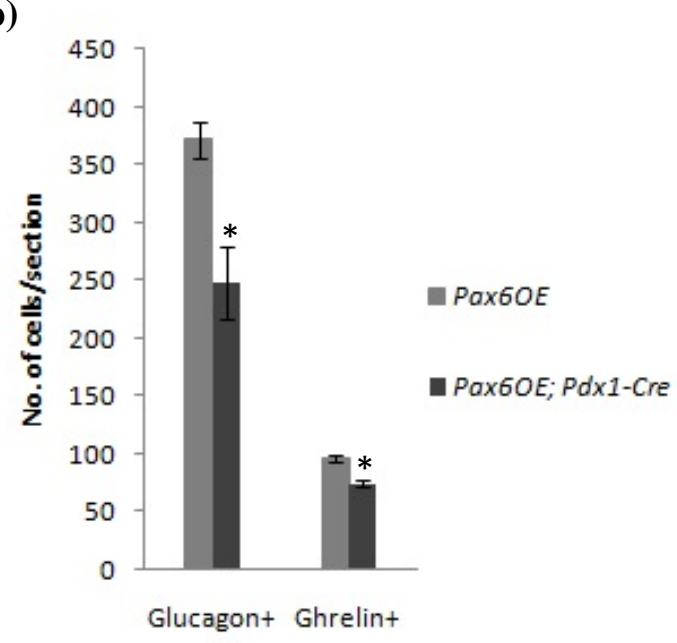

Figure 3.40: Changes in the glucagon ${ }^{+}$, ghrelin ${ }^{+}$, and $\mathbf{P P}^{+}$cell population in the pancreata of Pax6OE; Pdx1-Cre mice. Quantification of glucagon ${ }^{+}$and $\mathrm{PP}^{+}$cell pouplation at 3 weeks of age (a), and glucagon ${ }^{+}$and ghrelin ${ }^{+}$cell pouplation at P0 (b), in Pax6OE control and Pax6OE; Pdx1-Cre mice (n=3). In Pax6OE; Pdx1-Cre pancreata, number of glucagon ${ }^{+}$, ghrelin ${ }^{+}$, and $\mathrm{PP}^{+}$cells is decreased. Error bars represent SEM; ${ }^{*} \mathrm{p}<0.05$. 


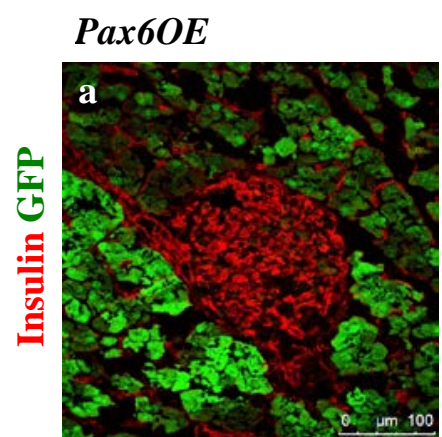

Pax6OE; Pdx1-Cre
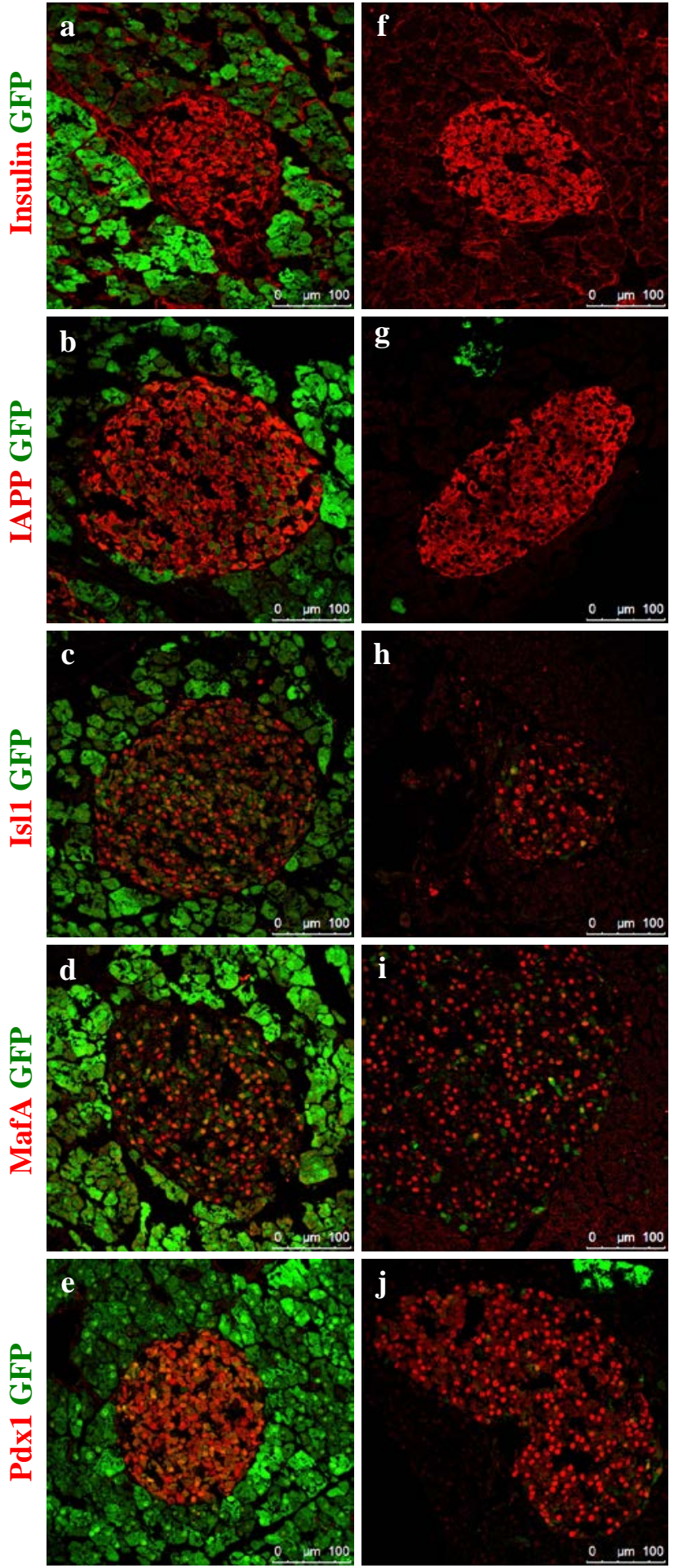

Figure 3.41: Expression of beta-cell related factors in Pax6OE; Pdx1-Cre islets. Immunofluorescence staining of pancreatic cryosections from 4 month old mice. As compared to the Pax6OE control islets, no change is detected in the expression of insulin (f), IAPP (g), Isl1 (h), MafA (i), and Pdx1 (j) in Pax6OE; Pdx1-Cre islets. 
population was not detectable in both Pax6OE and Pax6OE; Pdx1-Cre mice. Therefore, to examine any possible difference we went to the early postnatal stage P0, that is a timepoint where ghrelin ${ }^{+}$cells are in generally higher in number in the islets (Wierup et al., 2004). By quantification at $P 0$, we found a small but sgnificant decrease in the number of ghrelin $^{+}$cells in the pancreata of Pax6OE; Pdx1-Cre mice as compared to the Pax6OE control mice (Figure 3.40b). Furthermore, the change in other endocrine cells at P0 was similar to that in 3 week old mice (data not shown). As Pdx1-Cre is active from the beginning of pancreatic development, we conclude that this reduction in the population of glucagon ${ }^{+}$, ghrelin ${ }^{+}$, and $\mathrm{PP}^{+}$cell population is probably due to the reduced number of progenitors initially specified to these cell types or due to the reduced proliferation during early development. However, at 3 weeks of age no difference in proliferation (determined by BrdU labeling) or apoptosis (determined by TUNEL staining) was detected (data not shown).

In case of Pax6OE; Ins-Cre mice, no phenotypic change was observed. As compared to the Pax6OE control mice, the number of insulin ${ }^{+}$cells, number of islets per section, as well as the proliferation in the insulin ${ }^{+}$cell population was not significantly changed (Figure 3.42). Furthermore, the expression of different beta-cell related factors including Pdx1, MafA, Glut2, and IAPP was also not changed in the Pax6OE; Ins-Cre mice as compared to the Pax6OE control mice (data not shown).

Lastly, in Pax6OE; Glu-Cre mice, reduction in the number of glucagon ${ }^{+}$cells was observed like the Pax6OE; Pdx1-Cre mice. On the contrary, the number of $\mathrm{PP}^{+}$cells was slightly increased (Figure 3.43). Rest of the endocrine cell population was not changed in these mice (data not shown).

If the pancreatic phenotype of Pax6OE; Pdx1-Cre, Pax6OE; Ins-Cre, and Pax6OE; Glu-Cre mice is compared, it seems that the development of alpha-cells is more sensitive to the level of Pax6 expression as compared to the development of beta-cells. 
a)

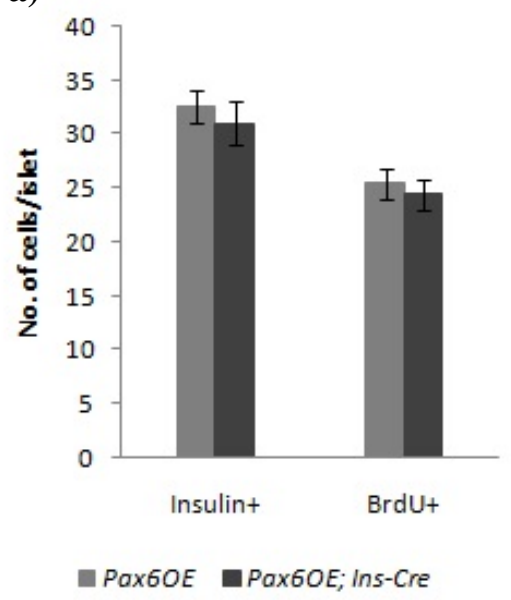

b)

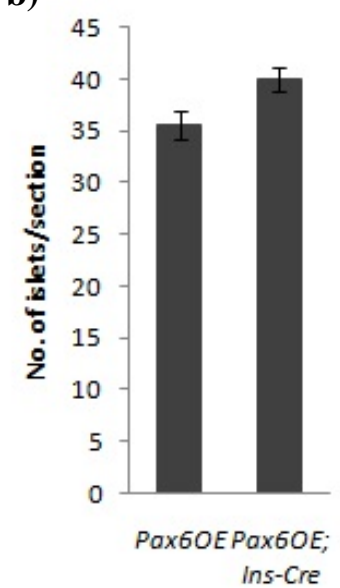

c)

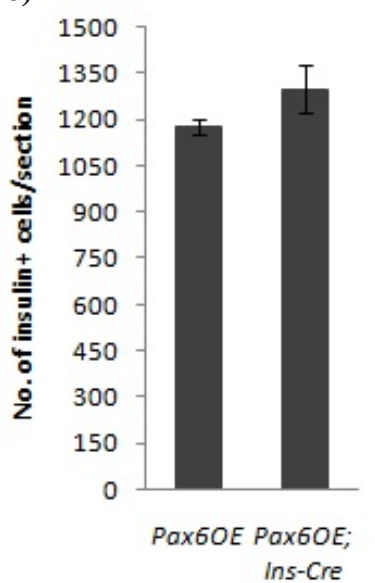

Figure 3.42: Pax6OE; Ins-Cre islets do not show any change in the beta-cell population. Quantification of insulin ${ }^{+}$and $\mathrm{BrdU}^{+}$cells (a), islets/section (b), and insulin ${ }^{+}$ cells/section (c) at 2 months of age shows no significant difference in Pax6OE vs. Pax6OE; Ins-Cre mice (n=3). Error bars represent SEM.

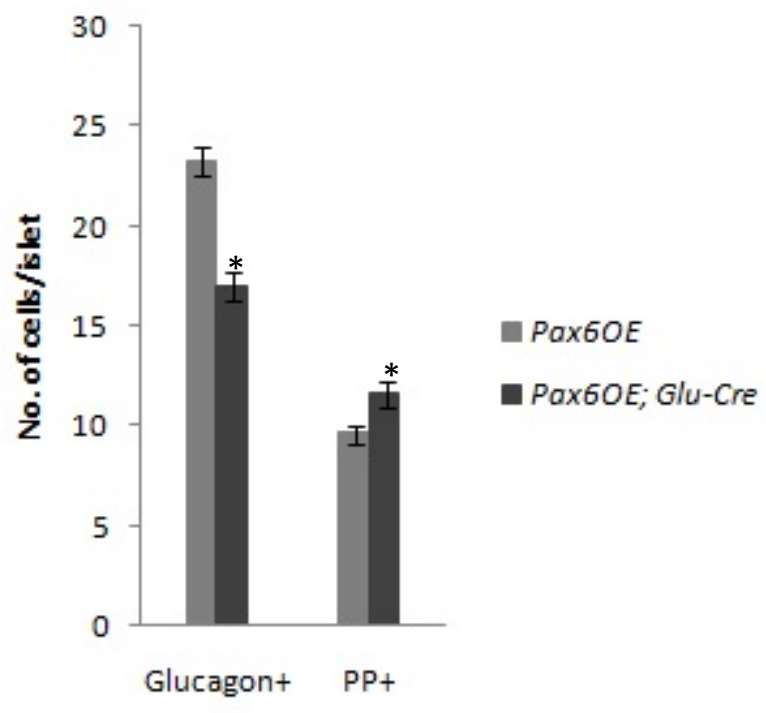

Figure 3.43: Changes in glucagon ${ }^{+}$and $\mathrm{PP}^{+}$cell pouplation in the islets of Pax6OE; Glu-Cre mice. Quantification of glucagon ${ }^{+}$and $\mathrm{PP}^{+}$cell pouplation in Pax6OE control and Pax6OE; Glu-Cre mice at 2 months of age (n=3). In Pax6OE; Glu-Cre islets, number of glucagon ${ }^{+}$cells is decreased and that of $\mathrm{PP}^{+}$cells is slightly increased. Error bars represent SEM; ${ }^{*} \mathrm{p}<0.05$. 


\section{Discussion}

Pax6 has been shown to play an important role in the maturation of pancreatic endocrine cells, especially alpha- and beta-cells. The crucial role of Pax6 in this process is evident by the fact that mice lacking a functional Pax6 protein in the pancreas die shortly after birth (St-Onge et al., 1997; Ashery-Padan et al., 2004). Pax6 is one of those transcription factors that are expressed in the endocrine pancreas during development and their expression further continues throughout the adult life-span. This persistent expression during postnatal life indicates the continuous requirement of Pax6 in the endocrine pancreas. This is supported by the fact that in both humans and mice, heterozygous Pax6 mutations lead to glucose intolerance with age (Yasuda et al., 2002; Wen et al., 2009). Hence, there has been a great interest in understanding the role of Pax6 in adult endocrine cell function.

In vitro Pax6 knockdown (KD) studies, in alpha- and beta-cells, have identified several downstream targets of Pax6 that are essential for the functional maturity of these cells (Katz et al., 2009; Gosmain et al., 2010; Gosmain et al., 2012a; Gosmain et al., 2012b). However, a detailed in vivo investigation of Pax6 knockout (KO) in the adult pancreas is lacking. As both classical and pancreas-specific conditional Pax6 KO mice die at early postnatal age, the only possible way to investigate the role of Pax6 in adult islets is by utilizing an inducible Cre line that can be activated during the adult life. Using this idea in a study that has been recently published, Hart et al. (2013) knocked out Pax6 in five month old mice. In this study, many of the same findings were recapitulated as those of the previous KO studies, including the loss of hormone expression and development of diabetes as well as the upregulation of ghrelin expression (Ashery-Padan et al., 2004; Heller et al., 2005). In this study a ubiquitous Cre line was used that led to the removal of Pax6 from all of the endocrine cell types. Therefore, it did not allow the study of a cell-type-specific effect.

Islets of Langerhans consist of five types of endocrine cells: alpha (glucagon $^{+}$), beta $\left(\right.$ insulin $\left.^{+}\right)$, delta (somatostatin $\left.{ }^{+}\right)$, PP (pancreatic-polypeptide ${ }^{+}$), and $\varepsilon\left(\right.$ ghrelin $\left.^{+}\right)$. Pax6 is expressed in all of them except for a subset of ghrelin ${ }^{+}$cells (Ashery-Padan et al., 2004; Kordowich et al., 2011). In our study we aimed at elucidating the role of Pax6 in adult alpha- and beta-cells in vivo by employing the Cre/loxP system (Sauer and Henderson, 1989; Rajewsky et al., 1996). Therefore, we utilized the Pax6 floxed mouse line together 
with an inducible Cre mouse line (RIP-CreER) for beta-cell-specific Pax6 ablation, and a non-inducible Cre mouse line (glucagon-Cre) for alpha-cell-specific Pax6 ablation (Ashery-Padan et al., 2000; Dor et al., 2004; Herrera, 2000). This allowed us to study the role of Pax6 in alpha- and beta-cells separately and without affecting the rest of the endocrine pancreas which was not the case in previous studies. We further incorporated the YFP reporter transgene (Srinivas et al., 2001) that allowed us to assess the recombination efficiency, to trace the fate of Pax6 KO cells, and to estimate the relative regeneration in each case.

Apart from the loss-of-function, another useful way to identify the role of a gene product in the developmental and/or functional context is via overexpression of that gene in the specific cell-type of interest (Prelich, 2012). In our study, we generated transgenic mice to conditionally overexpress Pax6. To see the effect of overexpression on pancreatic development, we utilized a wider range of expression domains including the whole pancreas, the beta-cells alone, and the alpha-cells alone. This was achieved by using Pdx1-Cre, insulin-Cre, and glucagon-Cre mouse lines, respectively (Gannon et al., 2000; Herrera, 2000). As the Pax6 overexpression phenotype was not lethal we used only noninducible Cre lines for Pax6 overexpression.

\subsection{Pax6 knockout in beta-cells}

\subsubsection{Hyperglycemia in beta-cell-specific Pax6 KO mice and the associated changes in the pancreatic endocrine cell population}

To knockout Pax6 in adult beta-cells, tamoxifen inducible RIP-CreER mouse line was employed (Dor et al., 2004). This Cre line gave nearly 95\% recombination efficiency. In accordance with this near complete removal of Pax6 from the beta-cell population, a prominent phenotype was observed. Pax6 $\mathrm{KO}$ in beta-cells resulted in severe hyperglycemia, emphasizing the requirement of Pax6 in beta-cells for the establishment of glucose homeostasis. Development of an overt diabetic phenotype in these mice is also in agreement with the previous Pax6 KO studies (Ashery-Padan et al., 2004; Hart et al., 2013). Consistent with the hyperglycemia, number of insulin ${ }^{+}$cells was decreased in the KO pancreata. At the same time all other endocrine cell types (glucagon ${ }^{+}$, somatostatin ${ }^{+}$, $\mathrm{PP}^{+}$, and ghrelin ${ }^{+}$) were increased in number. Decrease in the number of insulin ${ }^{+}$cells and increase in the number of glucagon ${ }^{+}$, somatostatin ${ }^{+}$, and $\mathrm{PP}^{+}$cells also occurs in 
streptozotocin-induced diabetic mouse models (Adeghate and Ponery, 2003; Zhang et al., 2012). Therefore, an increase in the population of glucagon ${ }^{+}$, somatostatin ${ }^{+}$, and $\mathrm{PP}^{+}$cells seems to be a general phenomenon related to the development of diabetes. However, increase in the ghrelin $^{+}$cell population is more unique to the Pax6 KO phenotype. The difference lies in the fact that in streptozotocin-induced diabetic models beta-cells die but in Pax6 KO mice beta-cells do not die. Instead, they just lose their mature differentiation status that may cause ghrelin upregulation in the previously insulin ${ }^{+}$cells (would be discussed later).

\subsubsection{Expression of beta-cell related transcription factors in beta-cell-specific Pax6 KO pancreata}

A detailed immunohistochemical analysis was performed to check the expression status of important beta-cell related factors. Among the beta-cell specific transcription factors, the expression of MafA was undetectable shortly after the KO. MafA is a transcription factor that specifically marks the mature beta cells and has been confirmed as a direct downstream target of Pax6 (Raum et al., 2010; Gosmian et al., 2012a). MafA, together with Pdx1 and Pax6 binds to the insulin promoter and controls its transcription (Sander et al., 1997; Matsuoka et al., 2007; Gosmain et al., 2012a). Indeed, the MafA deficient mice also develop glucose intolerance and diabetes mellitus (Zhang et al., 2005). Therefore, the loss of Pax6 and the concomitant loss of MafA in beta-cells can explain the reduced insulin synthesis that results into diabetes later.

Pdx1 and Nkx6.1 are two more beta-cell specific transcription factors in the adult islet. Pdx1 is important for insulin expression and mature beta-cell function, and its loss from beta-cells can lead to diabetes (Ahlgren et al., 1998; Matsuoka et al., 2007). In some studies, Pdx1 has been shown to be downregulated after Pax6 KO/KD (Heller et al., 2005; Gosmain et al., 2012a; Hart et al., 2013). On the contrary, it was still found to be expressed after Pax6 KO in another study (Ashery-Padan et al., 2004). In our beta-cellspecific Pax6 KO, Pdx1 expression was not changed even at 4.5 months after KO induction. However, as we have seen in our study the expression of Pdx1 alone is not sufficient to rescue the mature beta-cell phenotype. Thus, Pdx1 needs to cooperate with other beta-cell related transcription factors, including Pax6, MafA, and Nkx6.1, to maintain the mature differentiation state of beta-cells. 
Nkx6.1 in beta-cells is required to maintain the beta-cell identity by repressing the expression of glucagon and $\operatorname{Arx}$ (Gauthier et al., 2007; Schaffer et al., 2013). A reduction in the Nkx6.1 expression has been reported in Pax6 KO/KD models (Gosmain et al., 2012a; Hart et al., 2013). In our study, there was a gradual loss of Nkx6.1 expression. Shortly after KO induction, Nkx6.1 was still detectable but after a long time its expression was lost. Nkx6.1 expression is also reduced in beta-cell-specific Pdx1 KO mice and in turn leads to the ectopic expression of glucagon in beta-cells (Ahlgren et al., 1998). However, in our beta-cell-specific Pax6 KO mice the loss of Nkx6.1 expression did not lead to upregulation of glucagon, because the expression of glucagon depends on the presence of Pax6 (Sander et al., 1997; Gosmain et al., 2010). Regarding Arx expression following $N k x 6.1 \mathrm{KO}$, Schaffer et al. (2013) showed that $N k x 6.1$ inactivation in endocrine precursors but not in beta-cells leads to the ectopic expression of Arx. Once beta-cells are specified, the repression of Arx is independent of Nkx6.1 and occurs via DNA methylation (Dhawan et al., 2011). In accordance with that, we did not observe the ectopic expression of Arx in Pax6-deficient beta-cells following Nkx6.1 downregulation (data not shown).

Nkx2.2 is another transcription factor that is expressed in beta-cells, and in a subset of alpha- and PP-cells, and is required for the proper differentiation of these cell types (Kordowich et al., 2011). Previous studies have shown that Nkx2.2 expression is not affected in the Pax6 KO pancreata (Ashery-Padan et al., 2004; Gosmain et al., 2012a; Hart et al., 2013). Accordingly, in our beta-cell specific Pax6 KO mice the expression of Nkx2.2 was not changed.

Isl1 and Rfx6 are general pancreatic endocrine specific transcription factors in the adult pancreas. Both of them are expressed in all types of endocrine cells in the islet (Ahlgren et al., 1997; Smith et al., 2010). In mouse, Isl1 is required for the maturation and expansion of islet endocrine cells (Du et al., 2009) and Rfx6 is required for the generation of these cells except for the PP-cells that can develop in the absence of Rfx6 (Smith et al., 2010). In beta-cell-specific Pax6 KO islets the expression of both Isl1 and Rfx6 was not affected. Isl1 has also been shown to be unaffected in the previous Pax6 KO studies (Ashery-Padan et al., 2004; Gosmain et al., 2012a; Hart et al., 2013) while Rfx6 has not been evaluated. 
Collectively, the expression profile of various beta-cell related transcription factors in Pax6 KO beta-cells indicates a loss of mature differentiation state (due to the absence of MafA and downregulation of Nkx6.1) but preservation of basic endocrine and partial beta-cell character (due to the presence of Isl1, Rfx6, Nkx2.2, and Pdx1).

\subsubsection{Defective glucose-stimulated insulin secretion in beta-cell-specific Pax6 KO pancreata}

Glucose-stimulated insulin secretion (GSIS) from beta-cells is a crucial step in the maintenance of glucose homeostasis. In order for the GSIS to take place, an efficient glucose metabolism is required in the beta-cells. Glut2 is a high capacity (high $\mathrm{Km}$ ) glucose transporter located in the membranes of beta-cells. At high blood glucose level, it allows an efficient transport of glucose into the cell (e.g., immediately following a meal). This, in turn, leads to an increased glucose metabolism and stimulated insulin secretion (Jensen et al., 2008; Kramer et al., 2009). Loss of GSIS is an important step in the development of type 2 diabetes and is associated with the loss of Glut2 (Thorens et al., 1992). Glut2 deficient mice develop hyperglycemia and show loss of the first phase of GSIS (Guillam et al., 1997). In our study, following Pax6 KO, beta-cells quickly lost the expression of Glut2. It took place in the first few days together with the loss of MafA. Loss of Glut2 has been shown in previous Pax6 KO studies as well (Ashery-Padan et al., 2004). Additionally, we observed that the loss of Glut2 occurred in the whole islet, including the YFP labeled Pax6-deficient cells as well as the YFP $^{-}$insulin ${ }^{+}$cells that still express Pax6. Furthermore, ChIP analysis did not show any binding of Pax6 with the Glut2 promoter. Collectively, these results suggest an indirect affect of Pax6 ablation on Glut2 expression. It has been shown before that the diabetic environment can result in the loss of Glut2 expression (Thorens et al., 1992). Therefore, it is quite possible that the loss of Glut2 expression in the islets of beta-cell-specific Pax6 KO mice also occurs in an indirect way through the development of hyperglycemia.

Another important factor involved in GSIS is glucagon-like peptide 1 (GLP-1). GLP1 acts through the GLP-1 receptor that is highly expressed on beta-cells. Following nutrient stimulation, GLP-1 is released from intestinal L cells and promotes the insulin secretion from beta-cells. Apart from the role of GLP-1 in GSIS, it has also been shown to promote beta-cell proliferation and neogenesis as well as insulin synthesis (MacDonald et al., 2002). Previously, an in vitro Pax6 KD study has shown Pax6 mediated control of 
GLP-1 receptor expression (Gosmain et al., 2012a). In agreement with that, we found that the expression of GLP-1 receptor was specifically lost in the Pax6-deficient beta-cells. Loss of both Glut2 and GLP-1 receptor shows an obvious defect in the GSIS in Pax6deficient beta-cells. Additionally, the loss of GLP-1 receptor mediated signaling may also explain the reduced insulin production and the lack of proliferation in these Pax6deficient beta-cells.

Previously, MafA has been shown as a regulator of glucose-stimulated insulin secretion (Zhang et al., 2005). As MafA expression is downregulated in beta-cell-specific Pax6 KO islets, the impairment in GSIS in these mice may also occur indirectly via Pax6MafA pathway.

\subsubsection{Defective proinsulin processing in beta-cell-specific Pax6 KO pancreata}

Insulin is synthesized as a prohormone in beta-cells. Proinsulin is then processed to generate mature insulin peptide. During this process C-peptide is released as a byproduct at an equimolar concentration. In case of defective proinsulin processing the ratio of proinsulin to C-peptide is increased (Vasic and Walcher, 2012). Beta-cells contain two prohormone convertases for insulin processing, PC1/3 and PC2. This is in contrast to the non-beta-cells of the islet that contain only PC2 (Marcinkiewicz et al., 1994). Although both PC1/3 and PC2 are involved, proinsulin processing is more severely affected in PC1/3 KO mice as compared to PC2 KO mice (Furuta et al., 1997; Zhu et al., 2002). We checked the expression of insulin, C-peptide, PC1/3, and PC2 to evaluate the effect of Pax6 KO on proinsulin processing. Within few weeks of Pax6 KO, a lot of beta-cells lost the expression of C-peptide. Some of these C-peptide negative beta-cells were also insulin negative but some continued to express insulin at low level. We speculate that this insulin reactivity was due to the presence of proinsulin that was not completely processed as indicated by the absence of C-peptide. Surprisingly, however, the expression of both PC1/3 and PC2 was unchanged in the C-peptide ${ }^{-}$insulin $^{+}$cells. PC1/3 and PC2 expression was also found in those cells that had completely lost the expression of insulin (C-peptide ${ }^{-}$ insulin` cells). Previous studies show some conflicting data on PC1/3 and PC2 expression. PC1/3 expression has been shown to be not changed in one study (Ashery-Padan et al., 2004) and lost in the other study (Hart et al., 2013) after Pax6 KO, while it was found to be 30\% decreased at protein level after in vitro Pax6 KD (Gosmain et al., 2012a). PC2 
expression was found to be not changed after Pax6 KO (Hart et al., 2013) and a 30\% decrease in protein content was found after in vitro Pax6 KD (Gosmain et al., 2012a ).

Activities of PC1/3 and PC2 are further modulated by the neuropeptides proSAAS and 7B2, respectively (Fortenberry et al., 2002). We found that both of these peptides were highly upregulated in Pax6 KO beta-cells. It has been shown before that proSAAS can inhibit the activity of PC1/3 and its overexpression in mice leads to diabetes and obesity (Wei et al., 2004). Moreover, proSAAS is directly repressed by Pax6 and its expression is increased in the Pax6 heterozygous null mutant mice (Liu et al., 2012). Therefore, the increased level of proSAAS expression is expected in Pax6 KO beta-cells and is a possible indicator of defective proinsulin processing in these cells. Interestingly, however, we found that the proSAAS expression is in generally higher in alpha-cells in the control islets and does not seem to be affected after alpha-cell-specific Pax6 ablation (data not shown). Thus, while Pax6 may repress the expression of proSAAS in beta-cells, it does not seem to do so in alpha-cells indicating that the proSAAS expression in alphavs. beta-cells is differentially regulated.

Unlike proSAAS, the effect of upregulated 7B2 is hard to explain. 7B2 is a chaperone required for the maturation and activity of PC2 (Westphal et al., 1999). At the same time, C-terminal peptide of 7B2 can inhibit PC2 activity in vitro but apparently not in vivo (Fortenberry et al., 1999). When overexpressed in a beta-cell line, 7B2 did not affect insulin production or secretion, although, it increased the secretion of active PC2 (Helwig et al., 2011). In another study, 7B2 upregulation was linked to obesity and increased PC2 activity without changing the level of PC2 processed peptides (Farber et al., 2008). On the other hand, 7B2 downregulation is found in human medulloblastomas indicating that proper expression of 7B2 is required to suppress the tumor growth and to maintain the neuronal differentiation state (Waha et al., 2007). Finally, in a very recent study, it was shown that 7B2 can also prevent the aggregation of proteins involved in the pathogenesis of Alzheimer's and Parkinson's disease, thereby improving the cell viability (Helwig et al., 2013).

Therefore, on one hand, the increased expression of 7B2 in Pax6 KO beta-cells may help to increase the PC2 activity that may increase proinsulin processing in the absence of PC1/3 activity. On the other hand, this increased 7B2 expression might also be a result of the immature differentiation status of these cells and help in their survival by acting as a 
chaperone. However, the exact role of upregulated 7B2 in Pax6 KO beta-cells remains elusive.

\subsubsection{Ratio of IAPP to insulin is changed in the beta-cell-specific Pax6 KO islets}

Islet amyloid polypeptide (IAPP) (also called amylin) is a peptide hormone produced in beta-cells and secreted together with insulin in a ratio of 1:100 (IAPP to insulin). IAPP is secreted in response to nutrient stimuli together with insulin and may play a role in the regulation of food intake, in the development of insulin resistance (by suppressing the peripheral glucose disposal), and in the inhibition of glucose-stimulated insulin secretion (Leighton and Cooper, 1988; Sowa et al., 1990; Dégano et al., 1993; Hull et al., 2004). At the same time, IAPP is also well known for its pathological role in the development of type 2 diabetes as a part of the amyloid deposits (Kahn et al., 1999). Similar to insulin, IAPP is synthesized as a prohormone (proIAPP). ProIAPP is processed by the same enzymes (PC1/3 and PC2) that process proinsulin. Therefore, a defect in the proinsulin processing can also affect the proIAPP processing. It has been suggested that the unprocessed form of IAPP is more prone to form the amyloid deposits that cause the damage to beta-cells in type 2 diabetes (Marzban et al., 2004).

We found that the expression of IAPP was not affected in the islets of beta-cellspecific Pax6 KO mice. Firstly, this would change the normal IAPP to insulin ratio (as insulin is decreased in the $\mathrm{KO}$ islets) that may negatively affect the insulin secretion and contribute to further impairment of beta-cell function. A change in the IAPP to insulin ratio may also contribute to insulin resistance. Secondly, as the proinsulin processing is defective in the Pax6 KO islets, proIAPP processing would also be defective due to the same processing machinery involved. This may lead to the accumulation of unprocessed form of IAPP that can cause additional damage to beta-cells.

\subsubsection{Long-term tracing of Pax6 KO beta-cells in relation to ghrelin expression}

Ashery-Padan et al. (2004) used hAP (human alkaline phosphatase) reporter in pancreasspecific conditional Pax6 KO mice to trace the Pax6 KO endocrine cells. They found that the number of glucagon and insulin expressing cells is decreased but that of somatostatin and PP expressing cells is unchanged in the KO pancreata. Additionally, the hAP labeled endocrine area was not significantly changed in the KO pancreata. This led them to conclude that the $\mathrm{KO}$ endocrine cells stay in the pancreas but do not undergo a fate 
change to any of the other pancreatic endocrine cells. It was published before the discovery of ghrelin expressing epsilon-cells in the pancreas (Prado et al., 2004). Later on, it was shown by Heller et al. (2005) that ghrelin expressing cells are increased in number in $\mathrm{Pax}^{\mathrm{sey} / \mathrm{sey}}$ pancreata. In this study, it was concluded that Pax6 antagonizes the formation of ghrelin ${ }^{+}$epsilon-cells.

In the wild-type pancreas, the number of ghrelin expressing cells is highest during the embryonic development. Subsequently, these cells decrease in number and finally become almost undetectable in the adult pancreas (Wierup et al., 2004; Hill et al., 2009). As both the Pax6 KO and Pax6 $6^{\text {sey/sey }}$ mice die shortly after birth (Hill et al., 1991; St-Onge et al., 1997; Ashery-Padan et al., 2004), it was only possible to analyze the ghrelin ${ }^{+}$cell population at the embryonic stage and the long term fate of the Pax6-deficient cells was not identified. Furthermore, due to the absence of Pax6 from the beginning of pancreatic development, the endocrine cells (especially alpha- and beta-cells) never attain the functional maturity. Therefore, an upregulation of ghrelin at this stage can suggest a possible developmental shift to the ghrelin expressing epsilon-cell fate.

Recently, Hart et al. (2013) carried out the whole-body knock out of Pax6 in the adult mice and found an increase in the ghrelin expressing cell population of the pancreas. In this study, most of the ghrelin ${ }^{+}$cells found were located at the periphery of the islets and were not shown to be co-positive for any of the other endocrine hormones. Therefore, the authors concluded that these ghrelin ${ }^{+}$cells belong to the standard epsilon-cell population.

In our study, we knocked out Pax6 specifically from the beta-cells while leaving the rest of the endocrine cell population unaffected. After the induction of $\mathrm{KO}$, ghrelin expression started to upregulate in beta-cells and gradually expanded as more and more Pax6-deficient cells started to produce ghrelin. Initially the Pax6 KO beta-cells expressed ghrelin together with insulin. At later stages, insulin expression disappeared from many cells converting them to ghrelin only expressing cells. By showing the expression of several other beta-cell related factors in ghrelin ${ }^{+}$cells we proved that these ghrelin ${ }^{+}$cells arise from beta-cells. This was additionally confirmed by YFP reporter tracing. Therefore, the ghrelin ${ }^{+}$cells in our beta-cell-specific Pax6 KO pancreata do not belong to the standard epsilon-cell population. Instead, they are the beta-cells that have lost their maturity and started to produce higher levels of ghrelin (Figure 4.1). 


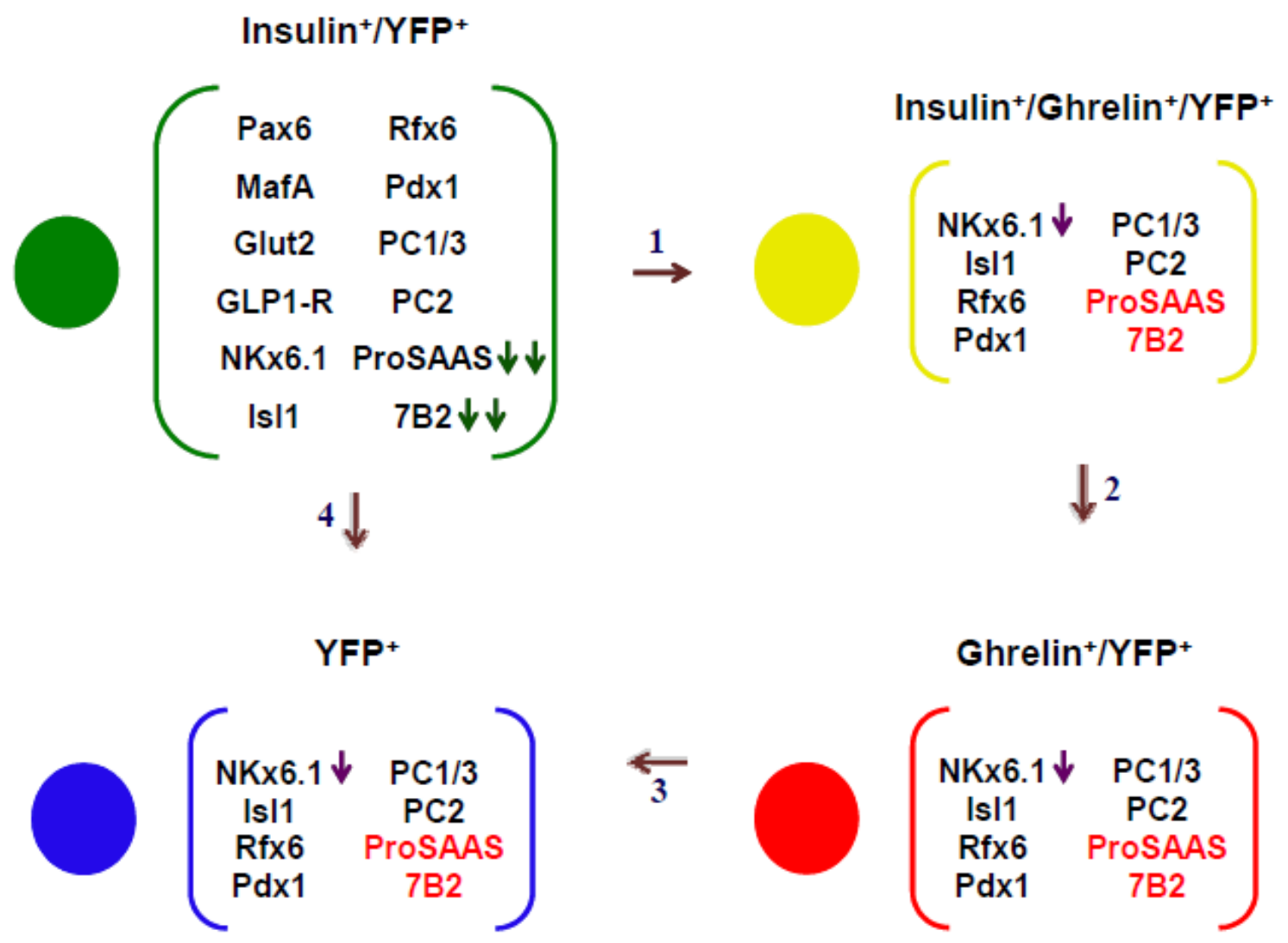

Figure 4.1: Schematic representation of the phenotypic change observed in Pax6deficient beta-cells. Beta-cells in the control mice express insulin, Pax6, Isl1, Rfx6, Pdx1, MafA, Nkx6.1, Glut2, GLP1-R, PC1/3, PC2, and very low levels of 7B2 and proSAAS. Following Pax6 ablation, the expression of MafA, Glut2, and GLP-1 receptor (GLP1-R) is lost, the expression of Nkx6.1 is reduced, the expression of Isl1, Rfx6, Pdx1, PC1/3, and PC2 is maintained, and the expression of proSAAS and 7B2 is upregulated. Along with that, Pax6-deficient cells gradually lose the expression of insulin and gain the expression of ghrelin. During this process these cells pass through a transient state of ghrelin-insulin co-expression before converting to ghrelin only expressing cells (arrows 1 and 2). Later on, some of these ghrelin expressing cells lose the expression of ghrelin as well (arrow 3). Some of the insulin ${ }^{+}$cells directly convert to insulin ${ }^{-}$cells after losing Pax6, without going through the ghrelin expression route (arrow 4). 
Moreover, we found that not all the beta-cells start to produce ghrelin after Pax6 ablation and many that do produce ghrelin stop to do so over long period of time. However, irrespective of the ghrelin expression these Pax6-deficient cells retain their endocrine and beta-cell like character as indicated by the presence of Rfx6 and Pdx1 expression, respectively. Therefore, even after losing the Pax6 expression beta-cells do not change their fate to ductal or exocrine cells that are the Pax6 negative population of cells in the pancreas. Lastly, we found that these Pax6-deficient beta-cells neither proliferate nor they undergo apoptosis. They stay as a quiescent population of immature beta-like-cells in the islets. The prevention from apoptosis can be attributed to the antiapoptotic effect of ghrelin and neurotensin (Coppola et al., 2008; Chung et al., 2008). Neurotensin is another hormone that was found to be upregulated in Pax6 KO islets (data not shown).

The role of ghrelin in the embryonic pancreas development is not clear. Ghrelin deficient mice have a completely normal development of endocrine and exocrine pancreas (Hill et al., 2009). Recently, Arnes et al. (2012) used lineage tracing to find the fate of ghrelin ${ }^{+}$cells in the wild-type pancreas. They found that ghrelin ${ }^{+}$cells mainly give rise to most of the alpha- and PP-cells in the islets. It was, thus, concluded that ghrelin ${ }^{+}$ cell population represents a multipotent progenitor population in the wild-type pancreas. From this it is also evident that ghrelin expression marks cells that have the potential to differentiate but have not fully differentiated yet, and when they do differentiate they lose the expression of ghrelin.

It could be, therefore, hypothesized that ghrelin expression marks a specific predifferentiation state of the cell. When beta-cells lose their functional maturity, they go back to a pre-differentiation embryonic-like state. This specific state is marked by an increased expression of ghrelin. However, it must be emphasized here that this predifferentiation state following Pax6 ablation is distinct from the normal immature state of beta-cells in the embryonic pancreas. The adult Pax6 KO beta-cells express neither MafA nor MafB but the early embryonic beta-cells express MafB before they start to express MafA. Furthermore, we never found the co-expression of ghrelin with insulin in the wildtype embryonic pancreas (data not shown). 
Finally, a rare population of ghrelin ${ }^{+}$cells in the beta-cell-specific Pax6 KO islets was not $\mathrm{YFP}^{+}$meaning that these cells do not arise from beta-cells and may represent the genuine epsilon-cell population.

\subsubsection{Regeneration of beta-cells in the beta-cell-specific Pax6 KO islets}

Beta-cells constitute a dynamic population that can undergo compensatory changes in relation to the varying metabolic needs. Increase in metabolic demand during pregnancy, obesity, or diabetes can induce an increase in beta-cell mass. This expansion of beta-cell mass may occur via increase in proliferation, decrease in apoptosis, increase in cell size and insulin secretion, and generation of new beta-cells from progenitors (neogenesis) or from other endocrine cells (transdifferentiation) (Ahmad, 2013).

Regeneration of beta-cells has been reported in various diabetic mouse models and can lead to a spontaneous recovery from diabetes (Nir et al., 2007; Thorel et al., 2010; Ahmad, 2013). After Pax6 ablation in beta-cells, we observed an overt diabetic phenotype. However, when followed over a long time a slight decrease in the blood glucose level was observed in young mice (KO induction at 3 weeks) but not in older mice (KO induction at 1.5 months). Nevertheless, a complete recovery to pre-KO level was never observed. By quantification, an increase in the number of YFP negative insulin $^{+}$cells was observed in the $\mathrm{KO}$ islets that shows expansion of insulin ${ }^{+}$cells that escaped recombination and/or insulin ${ }^{+}$cells that appeared as a result of neogenesis. Again this increase was greater at younger age compared to the older age. This difference in young vs. old age is explained by the higher basal proliferation in 3 week old mice compared to 1.5 month old ones.

Apart from proliferation some beta-cells may arise from alternative sources such as via transdifferentiation of alpha-cells (Thorel et al., 2010). Occasionally, we observed some Pdx1 positive alpha-cells in beta-cell-specific Pax6 KO islets. As Pdx1 is a beta-cell specific transcription factor, its presence in alpha-cells can show an intermediate transition state towards beta-cell generation. Transdifferentiation of alpha- to beta-cells has bee shown as the primary mechanism of beta-cell generation after extreme beta-cell loss (Thorel et al., 2010). Additionally, we observed some MafB positive beta-cells in the beta-cell-specific Pax6 KO islets. MafB is an alpha-cell specific transcription factor that is also expressed in immature beta-cells during embryonic development (Artner et al., 
2007). Presence of MafB in some beta-cells can, therefore, indicate their immature state and new emergence.

In conclusion, some level of beta-cell regeneration does occur after Pax6 ablation from beta-cells. This regeneration involves proliferation of pre-existing beta-cells and/or generation of some new beta-cells through other sources.

\subsection{Pax6 knockout in alpha-cells}

\subsubsection{Loss of glucagon expression in Pax6 KO alpha-cells}

Using the non-inducible glucagon-Cre mouse line (Herrera, 2000), we achieved almost $70 \%$ recombination efficiency as indicated by the YFP reporter expression. This means that at a given time-point nearly $70 \%$ of the alpha-cells would have lost Pax6. Labeling with YFP reporter also allowed us to precisely identify and track the Pax6 KO alphacells. The KO alpha-cells did not express glucagon as revealed by immunostaining and were also negative for the expression of MafB. In adult islets, MafB is an alpha-cell specific transcription factor that is a direct downstream target of Pax6 and together with Pax6 it controls the transcription of Proglucagon gene (Artner et al., 2006; Gosmain et al., 2010). Therefore, the loss of Pax6 and then MafB explains the inability of these cells to synthesize glucagon.

\subsubsection{Ghrelin upregulation in Pax6 KO alpha-cells}

It has been previously shown that ghrelin is upregulated in Pax6 KO pancreata. However, the exact origin of these ghrelin ${ }^{+}$cells has not been clearly identified (Heller et al., 2005; Kordowich et al., 2011). Similar to these preceding studies, we found an increased expression of ghrelin in the mutant islets. Lineage tracing with YFP reporter confirmed that these ghrelin ${ }^{+}$cells are originated from previously glucagon ${ }^{+}$cells. This conclusion was strengthened by two more observations. Firstly, the presence of some reporter labeled ghrelin-glucagon co-positive cells along with the reporter labeled ghrelin-only ${ }^{+}$cell population. Secondly, the expression of Arx, a marker of alpha-cell lineage (Collombat et al., 2003, 2005, 2007), in the ghrelin ${ }^{+}$cells. Thus, alpha-cells lost the expression of glucagon following Pax6 ablation and started to produce ghrelin (Figure 4.2). These ghrelin expressing cells were distinct from the ghrelin ${ }^{+}$cells that emerge in the beta-cellspecific Pax6 KO islets as the later did not express Arx (data not shown). 


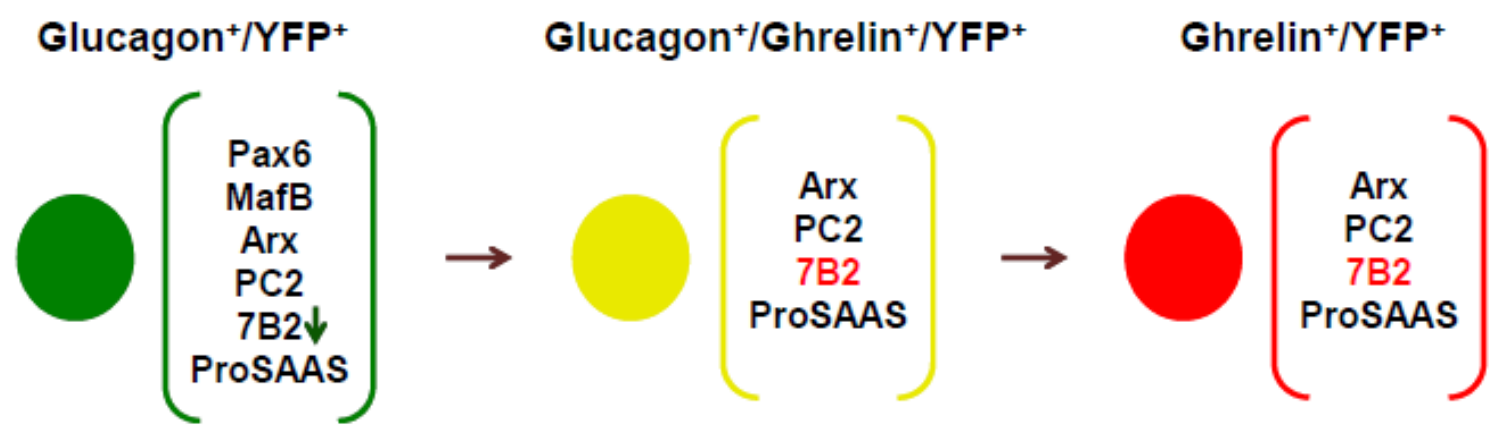

Figure 4.2: Schematic representation of the phenotypic change observed in Pax6deficient alpha-cells. Alpha-cells in the control mice express glucagon, Pax6, MafB, Arx, PC2, proSAAS, and low level of 7B2. Following Pax6 ablation, the expression of glucagon and MafB is lost, the expression of Arx, PC2, and proSAAS is maintained, and the expression of 7B2 is upregulated. Along with that ghrelin expression is highly upregulated in the Pax6-deficient cells, and the cells transiently pass through a glucagonghrelin co-positive state before they completely convert into ghrelin expressing cells. 
Additionally, we observed some $\mathrm{YFP}^{-}$ghrelin $^{+}$cells in the alpha-cell-specific Pax6 $\mathrm{KO}$ islets that were also Arx ${ }^{+}$. Hence, a rare population of ghrelin ${ }^{+}$cells in the KO islets is not coming from alpha-cells and may represent the cells from genuine epsilon-cell population.

We found the upregulation of ghrelin expression in both Pax6-deficient alpha- and beta-cells. This suggests that the molecular mechanism behind this upregulation of ghrelin is probably similar in both of these cell types. By ChIP assay, we found no interaction of Pax6 with the ghrelin promoter. Therefore, it seems that the upregulation of ghrelin is not directly due to the loss of Pax6-mediated repression of ghrelin expression. It is rather due to the activation of a pathway that is shared between alpha- and beta-cells and is activated when these cells lose their mature differentiation status.

\subsubsection{B2 upregulation in Pax6 KO alpha-cells}

In alpha-cells, glucagon is initially synthesized as proglucagon, a precursor form that is processed by the enzyme prohormone convertase 2 (PC2) to generate the mature glucagon peptide (Furuta et al., 1997). Prohormone convertase 2 is itself synthesized as a proprotein (pro-PC2) that requires the presence of neuroendocrine protein 7B2 for its maturation and activation (Westphal et al., 1999). Previous in vitro studies have shown that proglucagon processing in the Pax6 knockdown alpha-cells is impaired due to the reduced expression of both PC2 and 7B2 (Katz et al., 2009). However, in contrast to this in vitro study we found no change in the PC2 immunoreactivity in Pax6 KO alpha-cells. Surprisingly, instead of being decreased the expression of 7B2 was highly upregulated in these cells. This increased expression of 7B2 in the KO cells might be in an effort to enhance the PC2 activity in response to the reduced hormone synthesis. This idea is supported by the in vitro study, where 7B2 overexpression in alpha-TC6 cells led to the increased production of glucagon (Helwig et al., 2011). Alternatively, this upregulation of 7B2 might be in relation to the differentiation status of Pax6-deficient cells as discussed in the beta-cell section.

As 7B2 is upregulated in both alpha- and beta-cell-specific Pax6 KO mouse models, this means that a common pathway is affected in both cell types that leads to upregulation of 7B2. This specific pathway is perhaps affected in the intact islet in vivo but not in the isolated cells in vitro. This may explain the difference of 7B2 expression following Pax6 ablation in vitro vs. in vivo. 


\subsubsection{Regeneration of alpha-cells in the alpha-cell-specific Pax6 KO islets}

As the function of glucagon is to increase the blood glucose level in response to hypoglycemia, a reduced blood glucose level is expected under conditions of glucagon deficiency (Gelling et al., 2003). In spite of the fact that Pax6 KO alpha-cells lose the expression of glucagon, we were not able to see any change in the blood glucose level. This can be explained by either the lower recombination efficiency and/or by the regeneration of alpha-cells. Indeed, as the recombination efficiency was $70 \%$, the rest of the $30 \%$ alpha-cells may produce enough glucagon to prevent any appreciable change in the blood glucose level. Interestingly, however, the total number of glucagon ${ }^{+}$cells was not significantly different in 1 month old control and knockout mice. In contrast to the total population, the number of YFP negative glucagon ${ }^{+}$cells was significantly increased in the $\mathrm{KO}$ islets. Hence, it is quite probable that alpha-cell regeneration did take place after the Pax6 KO. Analysis of later stages (3 month and 8 month) showed a decrease in the alpha-cell regeneration at older age (8 months) but not at the middle age (3 months). However, even the older mice that showed no regeneration were healthy, possibly due to the remaining unrecombined population of alpha-cells. Regeneration of alpha-cells has been demonstrated in other models of glucagon deficiency as well. Mice that lack glucagon receptor or PC2 show a massive alpha-cell hyperplasia (Furuta et al., 1997; Gelling et al., 2003). As compared to these models, the regeneration that we observe is only moderate, and this is because of the inability to achieve $100 \%$ recombination in alpha-cells.

As the glucagon-Cre line used was not inducible, it was not possible to define the age of Pax6-deficient alpha-cells. At each of the age analyzed, the number of YFP labeled cells was significantly less in the alpha-cell-specific Pax6 KO islets compared to the control islets. In contrast to that, same numbers of YFP labeled cells were detected at either 1 month or 8 months of age in the alpha-cell-specific Pax6 KO islets. If we assume that glucagon ${ }^{+}$cells regenerate in the $\mathrm{KO}$ islet and convert to ghrelin ${ }^{+}$cells following the activation of Cre, this should lead to an increase in the ghrelin ${ }^{+} \mathrm{YFP}^{+}$cell population. Otherwise, some of the ghrelin ${ }^{+} \mathrm{YFP}^{+}$cells should disappear e.g., via apoptosis. However, apoptosis was not detectable in the KO islets as determined by the TUNEL staining (data not shown). Therefore, it remains unclear that what happens to the ghrelin ${ }^{+}$ cells that arise from alpha-cells. 


\subsection{Comparison of Pax6 KO in alpha- and beta-cells}

In both alpha- and beta-cells, the ablation of Pax6 results in the loss of mature differentiation status and upregulation of ghrelin expression. However, the original character of the cell type is maintained in each case as evident by the continued expression of Arx in Pax6-deficient alpha-cells and that of Pdx1 in Pax6-deficient betacells. Similarly, the ghrelin expressing cells in each type of knockout pancreata are distinct from each other. Ghrelin expressing cells in beta-cell-specific Pax6 KO islets express Pdx1 and remain negative for Arx and glucagon expression. On the other hand, ghrelin expressing cells in alpha-cell-specific Pax6 KO islets express Arx and never show co-expression with insulin. Moreover, the alpha-cell derived ghrelin ${ }^{+}$cells are located at the periphery of the islets that is the normal location of alpha-cells in mouse islets. In contrast to that, the beta-cell derived ghrelin ${ }^{+}$cells are dispersed throughout the islet.

In the normal mouse islet, beta-cells form the core of the islet and other endocrine cell types stay at the periphery (Brissova et al., 2005). As compared to the control mice, the islet architecture in beta-cell-specific Pax6 KO mice is slightly disturbed as different types of endocrine cells are sometimes mixed into each other. Additionally, the number of glucagon, somatostatin, and PP producing cells is increased in these islets. However, in alpha-cell-specific Pax6 KO mice no such disturbance is observed. The number of insulin, somatostatin, and PP producing cells is not changed (data not shown) and the islet structure remains intact. For the comparison of cell-type-specific changes in both types of KO mice, see Figures 4.1 and 4.2.

\subsection{Pax6 overexpression}

To date, there is only one in vivo study of Pax6 overexpression done in pancreas. In this study, Yamaoka et al. (2000) overexpressed Pax6 directly under Pdx1 and insulin promoter. The phenotype was variable with many animals developing diabetes but not all. They found reduction in the number of insulin ${ }^{+}$cells due to increased apoptosis. Additionally, when overexpressed under $P d x 1$ promoter, Pax6 caused hypoplasia of the pancreatic exocrine tissue. One disadvantage of overexpressing Pax6 in this way is that both the $P d x 1$ and insulin promoters would be subject to regulation inside beta-cells that may lead to variation in the phenotype. 
We decided to repeat the overexpression of Pax6 in a different way. In our system Pax6 was overexpressed under CAG promoter that provides constitutive expression and, therefore, is less sensitive to cellular state. However, this overexpression is dependent on Cre/loxP system and can be activated in the desired cell type. Along with the $P d x 1$ (whole pancreas), and insulin (beta-cells) expression domains we overexpressed Pax6 in the glucagon (alpha-cells) expression domain too. Pax6OE mice in any domain did not develop diabetes. Difference in proliferation or apoptosis of beta-cells was also not detectable. However, like this previous study we also found hypoplasia of the exocrine pancreas in Pax6OE; Pdx1-Cre mice. Furthermore, we found decrease in the glucagon ${ }^{+}$, ghrelin ${ }^{+}$, and $\mathrm{PP}^{+}$cell population when Pax6 was overexpressed under $P d x 1$ expression domain indicating that compared to insulin $^{+}$or somatostatin $^{+}$cell population, development of these three cell types is more sensitive to the level of Pax6.

In another study by Kredo-Russo et al. (2012), miR-7 knockdown lead to indirect overexpression of Pax6. In this case also the number of ghrelin ${ }^{+}$cells was decreased. However, in contrast to our study, the number of both the insulin ${ }^{+}$and glucagon ${ }^{+}$cells was increased. This difference may arise from other targets that are affected by miR-7 knockdown along with Pax6. Secondly, the study was performed in explant culture that may behave a bit different from the real in vivo system.

\subsection{Comparison of the Pax6 pancreatic phenotype in relation to Pax6 function in brain and eye}

Apart from pancreas, Pax6 also plays essential roles in the development of brain and eye (Callaerts et al., 1997). However, the exact function of Pax6 in different tissues as well as in the different cell types of the same tissue can be highly variable. Without going into extensive detail we will note down few interesting examples from eye and brain and compare them with our pancreatic Pax6 KO /OE phenotype.

During eye development, Pax6 is required in the retinal progenitor cells (RPCs) for the completion of retinal neurogenesis. When Pax6 is deleted from the late phase optic cup after e10.5, a dual function of Pax6 is revealed. In the peripheral RPCs, loss of Pax6 results in reduced proliferation and beginning of early differentiation that never attains maturity. In the central RPCs, loss of Pax6 leads to a cell-fate change from retinal lineage to amacrine interneurons (Oron-Karni et al., 2008). Along with the retinal neurogenesis, 
Pax6 is also required for lens fiber cell differentiation. Here the presence of Pax6 is essential for the early phase of differentiation while downregulation of Pax6 is required for the last stage of lens differentiation (Shaham et al., 2012).

In brain, Pax6 is highly expressed in cortical radial glial cells (neural progenitors). In the absence of Pax6, neural progenitor pool is reduced due to increased cell-cycle exit and premature start of neuronal differentiation (Quinn et al., 2007). Pax6 is also expressed in the developing and postnatal astrocytes that originate from neural progenitors. Cultured astrocytes from $\mathrm{Pax}^{\text {sey/sey }}$ mice are not fully differentiated and show increased proliferation. Furthermore, the PI3K/Akt pathway, that enhances cell survival and reduces apoptosis, is also activated in these astrocytes as indicated by the increased activity of Akt (Sakurai and Osumi, 2008).

In spite of the variation, it seems that Pax6 derives successful differentiation of the respective cell types. Especially as compared to the Pax $6^{\text {sey/sey }}$ astrocyte phenotype, we also observed a loss of differentiation in the Pax6 KO alpha- and beta-cells. Though in our case there was decrease in proliferation rather than increase, that is more similar to the Pax6 KO in peripheral RPCs in the eye. Moreover, ghrelin that is upregulated in alpha- and beta-cells after Pax6 KO, may provide cell survival and anti-apoptotic affect by activating PI3K/Akt pathway (Chung et al., 2008).

Pax6 gene dosage is important during development. Like the reduced dosage, an overdosage of Pax6 also leads to developmental defects. However, again the effect of Pax6 overexpression can vary in different cell types at different time points of development. In eye, overexpression of Pax6 leads to defective iris, ciliary body, and retinal development without inducing apoptosis (Manuel et al., 2008; Davis et al., 2009). In brain, it was shown that Pax6 overexpression causes apoptosis in a set of cortical progenitors. Progenitors that have higher endogenous level of Pax6 expression are more resistant to the effect of Pax6 overexpression. Additionally, overexpression of Pax6 in postmitotic neurons does not cause apoptosis (Berger et al., 2007). Therefore, it seems that the sensitivity of different cell types to Pax6 dosage is different.

In our study, Pax6 overexpression in the pancreas did not cause apoptosis in the adult pancreas. In the exocrine pancreas, slight hypoplasia was observed. In the endocrine pancreas, glucagon ${ }^{+}$and $\mathrm{PP}^{+}$cells were reduced in number. Therefore, at the level of 
Pax6 expression achieved in these transgenic mice, the development of pancreas does not seem to be drastically affected.

Lastly, it is important to mention that some of the Pax6 target genes are shared among forebrain, lens, and beta-cells. In a recent study, Xie et al. (2013) utilized ChIP-chip approach to identify the targets of Pax6 in forebrain, lens, and beta-cells. Interestingly, they found that 133 target promoters were shared between all three tissue sources. Furthermore, MafA, MafB, Isl1, and Pcsk1n (proSAAS) that are expressed in the endocrine pancreas are also expressed in the eye and regulated by Pax6 (Xie et al., 2013). MafA and MafB are important for the alpha- and beta-cell development/maturation and are also required for lens development (Reza et al., 2002; Cvekl et al., 2004; Zhang et al., 2005; Artner et al., 2007). However, only about 3\% of the Pax6-bound promoters are shared between lens, forebrain, and beta-cells (Xie et al., 2013). This means that apart from having some similarity, these three tissue types are only distantly related. 


\section{Summary}

Pax6, a transcription factor from the paired-box family is expressed in the eye, central nervous system, olfactory system, and pancreas, and plays an important role in the development of these tissues (Shaham et al., 2012). In pancreas, Pax6 is required for the development and function of the endocrine pancreas especially alpha- and beta-cells (StOnge et al., 1997; Ashery-Padan et al., 2004; Gosmain et al., 2010; Gosmain et al., 2012a). However, as the Pax6 knockout mice die shortly after birth, it does not allow for the elucidation of Pax6 function in adult endocrine pancreas in vivo. Secondly, a celltype-specific effect of Pax6 ablation cannot be established if Pax6 is ablated from the whole pancreas.

For this purpose, we decided to generate Pax6 konckout (KO) in alpha- and betacells, separately. Additionally, we used a tamoxifen-inducible Cre line to ablate Pax6 from beta-cells in the adult mice. In parallel to that, we overexpressed Pax6 in the whole pancreas, in beta-cells alone, and in the alpha-cells alone. Following results were obtained in this study:

1. The beta-cell-specific Pax6 KO mice developed an overt diabetes in a short timeperiod after tamoxifen induction. In pancreas, the number of insulin ${ }^{+}$cells was decreased and that of ghrelin ${ }^{+}$cells increased. Lineage tracing with YFP reporter confirmed the origin of these ghrelin ${ }^{+}$cells from the insulin ${ }^{+}$cells.

2. Following Pax6 ablation, beta-cells lost their mature differentiation status and function. This was evident by the reduced expression of MafA and Nkx6.1, two mature beta-cell-specific transcription factors. Moreover, Pax6-deficient beta-cells were defective in insulin synthesis and glucose-stimulated insulin secretion, indicated by the absence of insulin, Glut2, and GLP-1 receptor expression. Finally, the proinsulin processing was also defective in Pax6-deficient beta-cells due to the increased expression of proSAAS that can inhibit PC1/3-mediated proinsulin processing.

3. Long-term tracing of Pax6-deficient beta-cells revealed that most of them continue to express ghrelin. These cells did not die or convert to any other cell type. Apparently, they maintained their endocrine and partial beta-cell-like character as shown by the continued expression of Rfx6 and Pdx1, that are 
endocrine-specific and beta-cell related transcription factors in the adult pancreas, respectively.

4. The alpha-cell-specific Pax6 KO mice remained healthy and did not show any change in blood glucose level. In pancreas, the number of glucagon ${ }^{+}$cells was unchanged at younger age but decreased at an older age. Ghrelin expression was also upregulated in alpha-cell-specific Pax6 KO islets. Lineage tracing with YFP reporter confirmed the origin of these ghrelin ${ }^{+}$cells from the glucagon ${ }^{+}$cells.

5. Following Pax6 ablation, alpha-cells lost their mature differentiation status and function. This was evident by the loss of glucagon and MafB expression. Pax6deficient alpha-cells, however, maintained their alpha-cell-like character as they continued to express Arx that is alpha-cell lineage determinant.

6. We found the increased expression of ghrelin in both Pax6-deficient alpha- and beta-cells. In each case, however, the ghrelin ${ }^{+}$cells maintained the character of the cell-type from which they originated. Alpha-cell derived ghrelin ${ }^{+}$cells continued to express Arx. On the other hand, beta-cell derived ghrelin ${ }^{+}$cells did not express Arx. Instead, they continued to express beta-cell related factors (e.g., Pdx1, IAPP, PC1/3).

7. Pax6 ablation from alpha- and beta-cells led to the loss of glucagon and insulin expression, respectively. This in turn resulted in the regeneration of the respective cell-type in each case. Regeneration capacity of both alpha- and beta-cells was higher at a younger age. However, in beta-cell-specific Pax6 KO mice the regeneration of beta-cells was not sufficient to allow for the complete recovery from diabetes even at a younger age.

8. Pax6 overexpressing mice remained healthy and did not show any change in the blood glucose level. In the pancreas-specific Pax6 overexpressing mice, slight hypoplasia of the exocrine pancreas was observed. In the endocrine pancreas, numbers of alpha-, PP-, and epsilon-cells were decreased and that of beta- and delta-cells not affected. In the beta-cell-specific Pax6 overexpressing mice, no change was observed in the pancreas. Lastly, in the alpha-cell-specific Pax6 overexpressing mice, again a decrease in the number of alpha-cells was observed. 
Therefore, the development of alpha-cells appears to be more sensitive to the level of Pax6 expression as compared to that of beta-cells. 


\section{References}

Adeghate E, Ponery A. (2003). Pancreatic peptides, neuropeptides and neurotransmitters in diabetes mellitus: a review. Int J Diabetes Metab 11: 1-6.

Afelik S, Chen Y, Pieler T. (2006). Combined ectopic expression of Pdx1 and Ptf1a/p48 results in the stable conversion of posterior endoderm into endocrine and exocrine pancreatic tissue. Genes Dev 20: 1441-1446.

Ahlgren U, Jonsson J, Jonsson L, Simu K, Edlund H. (1998). Beta-cell-specific inactivation of the mouse Ipf1/Pdx1 gene results in loss of the beta-cell phenotype and maturity onset diabetes. Genes Dev 12: 1763-1768.

Ahlgren U, Pfaff SL, Jessell TM, Edlund T, Edlund H. (1997). Independent requirement for ISL1 in formation of pancreatic mesenchyme and islet cells. Nature 385: 257-260.

Ahmad, Z. (2013). Pancreatic regeneration in the face of diabetes. In H. Baharvand and N. Aghdami (Eds.), Regenerative medicine and cell therapy. (pp. 169-201). New York: Springer.

Akiyama H, Kim JE, Nakashima K, Balmes G, Iwai N, Deng JM, Zhang Z, Martin JF, Behringer RR, Nakamura T, de Crombrugghe B. (2005). Osteo-chondroprogenitor cells are derived from Sox9 expressing precursors. Proc Natl Acad Sci USA 102: 14665-14670.

Apelqvist A, Li H, Sommer L, Beatus P, Anderson DJ, Honjo T, Hrabe de Angelis M, Lendahl U, Edlund H. (1999). Notch signalling controls pancreatic cell differentiation. Nature 400: 877881.

Arnes L, Hill JT, Gross S, Magnuson MA, Sussel L. (2012). Ghrelin expression in the mouse pancreas defines a unique multipotent progenitor population. PLoS One 7: e52026.

Arnold SJ, Robertson EJ. (2009). Making a commitment: cell lineage allocation and axis patterning in the early mouse embryo. Nat Rev Mol Cell Biol 10: 91-103.

Artner I, Blanchi B, Raum JC, Guo M, Kaneko T, Cordes S, Sieweke M, Stein R. (2007). MafB is required for islet beta-cell maturation. Proc Natl Acad Sci USA 104: 3853-3858.

Artner I, Le Lay J, Hang Y, Elghazi L, Schisler JC, Henderson E, Sosa-Pineda B, Stein R. (2006). MafB: an activator of the glucagon gene expressed in developing islet alpha- and beta-cells. Diabetes 55: 297-304.

Ashery-Padan R, Zhou X, Marquardt T, Herrera P, Toube L, Berry A, Gruss P. (2004) Conditional inactivation of Pax6 in the pancreas causes early onset of diabetes. Dev Biol 269: 479-488.

Ashery-Padan R, Marquardt T, Zhou X, Gruss P. (2000). Pax6 activity in the lens primordium is required for lens formation and for correct placement of a single retina in the eye. Genes Dev 14: 2701-2711.

Banerjee A, Onyuksel H. (2012). Human pancreatic polypeptide in a phospholipid-based micellar formulation. Pharm Res 29: 1698-1711.

Bansal P, Wang Q. (2008). Insulin as a physiological modulator of glucagon secretion. Am J Physiol Endocrinol Metab 295: E751-761.

Bayha E, Jorgensen MC, Serup P, Grapin-Botton, A. (2009). Retinoic acid signaling organizes endodermal organ specification along the entire antero-posterior axis. PLoS One 4: e5845. 
Berger J, Berger S, Tuoc TC, D'Amelio M, Cecconi F, Gorski JA, Jones KR, Gruss P, and Stoykova A. (2007). Conditional activation of Pax6 in the developing cortex of transgenic mice causes progenitor apoptosis. Development 134: 1311-1322.

Bort R, Martinez-Barbera JP, Beddington RS, Zaret KS. (2004). Hex homeobox gene-dependent tissue positioning is required for organogenesis of the ventral pancreas. Development 131: 797806.

Brissova M, Fowler MJ, Nicholson WE, Chu A, Hirshberg B, Harlan DM, Powers AC. (2005). Assessment of human pancreatic islet architecture and composition by laser scanning confocal microscopy. J Histochem Cytochem 53: 1087-1097.

Buchanan TA, Xiang AH. (2005). Gestational diabetes mellitus. J Clin Invest 115: 485-491.

Buckingham M, Relaix F. (2007). The role of Pax genes in the development of tissues and organs: Pax3 and Pax7 regulate muscle progenitor cell functions. Annu Rev Cell Dev Biol 23: 645-673.

Burlison JS, Long Q, Fujitani Y, Wright CV, Magnuson MA. (2008). Pdx1 and Ptf1a concurrently determine fate specification of pancreatic multipotent progenitor cells. Dev Biol 316: 74-86.

Callaerts P, Halder G, Gehring WJ. (1997). PAX-6 in development and evolution. Annu Rev Neurosci 20: 483-532.

Chanclón B, Martínez-Fuentes AJ, Gracia-Navarro F. (2012). Role of SST, CORT and ghrelin and its receptors at the endocrine pancreas. Front Endocrinol (Lausanne) 3: 114.

Chanoine JP, Wong AC. (2004). Ghrelin gene expression is markedly higher in fetal pancreas compared with fetal stomach: effect of maternal fasting. Endocrinology 145: 3813-3820.

Chen Y, Pan FC, Brandes N, Afelik S, Solter M, Pieler T. (2004). Retinoic acid signaling is essential for pancreas development and promotes endocrine at the expense of exocrine cell differentiation in Xenopus. Dev Biol 271: 144-160.

Chung H., Seo S., Moon M., Park S. (2008). Phosphatidylinositol-3-kinase/Akt/glycogen synthase kinase-3b and ERK1/2 pathways mediate protective effects of acylated and unacylated ghrelin against oxygen-glucose deprivation-induced apoptosis in primary rat cortical neuronal cells. J Endocrinol 198: 511-521.

Cleaver, O. and Melton, D. A. (2005). Development of the endocrine pancreas. In C. R. Kahn et al. (Eds.), Joslin's diabetes mellitus. (pp. 21-40). Boston, MA: Lippincott Williams \& Wilkins.

Collombat P, Hecksher-Sørensen J, Broccoli V, Krull J, Ponte I, Mundiger T, Smith J, Gruss P, Serup P, Mansouri A. (2005). The simultaneous loss of Arx and Pax4 genes promotes a somatostatin-producing cell fate specification at the expense of the alpha- and beta-cell lineages in the mouse endocrine pancreas. Development 132: 2969-2980.

Collombat P, Mansouri A, Hecksher-Sorensen J, Serup P, Krull J, Gradwohl G, Gruss P. (2003). Opposing actions of Arx and Pax4 in endocrine pancreas development. Genes Dev 17: 25912603.

Collombat P, Hecksher-Sørensen J, Krull J, Berger J, Riedel D, Herrera PL, Serup P, Mansouri A. (2007). Embryonic endocrine pancreas and mature beta-cells acquire alpha- and PP-cell phenotypes upon Arx misexpression. J Clin Invest 117: 961-970. 
Collombat P, Hecksher-Sørensen J, Serup P, Mansouri A. (2006). Specifying pancreatic endocrine cell fates. Mech Dev 123: 501-512.

Collombat P, Xu X, Ravassard P, Sosa-Pineda B, Dussaud S, Billestrup N, Madsen OD, Serup P, Heimberg H, Mansouri A. (2009). The ectopic expression of Pax4 in the mouse pancreas converts progenitor cells into alpha and subsequently beta-cells. Cell 138: 449-62.

Coppola T, Béraud-Dufour S, Antoine A, Vincent JP, Mazella J. (2008). Neurotensin protects pancreatic beta-cells from apoptosis. Int J Biochem Cell Biol 40: 2296-2302.

Cvekl A, Yang Y, Chauhan BK, Cveklova K. (2004). Regulation of gene expression by Pax6 in ocular cells: a case of tissue-preferred expression of crystallins in lens. Int J Dev Biol 48: 829844.

Dames P, Puff R, Weise M, Parhofer KG, Göke B, Götz M, Graw J, Favor J, Lechner A. (2010). Relative roles of the different Pax6 domains for pancreatic alpha-cell development. BMC Dev Biol 10: 39.

Daneman D. (2006). Type 1 diabetes. Lancet 367: 847-858.

Dansault A, David G, Schwartz C, Jaliffa C, Vieira V, de la Houssaye G, Bigot K, Catin F, Tattu L, Chopin C, Halimi P, Roche O, Van Regemorter N, Munier F, Schorderet D, Dufier JL, Marsac C, Ricquier D, Menasche M, Penfornis A, Abitbol M. (2007). Three new PAX6 mutations including one causing an unusual ophthalmic phenotype associated with neurodevelopmental abnormalities. Mol Vis 13: 511-523.

Davis N, Yoffe C, Raviv S, Antes R, Berger J, Holzmann S, Stoykova A, Overbeek PA, Tamm ER, Ashery-Padan R. (2009). Pax6 dosage requirements in iris and ciliary body differentiation. Dev Biol 333: 132-142.

Dégano P, Silvestre RA, Salas M, Peiró E, Marco J. (1993). Amylin inhibits glucose-induced insulin secretion in a dose-dependent manner. Study in the perfused rat pancreas. Regul Pept 43: 91-96.

Dessimoz J, Opoka R, Kordich JJ, Grapin-Botton A, Wells JM. (2006). FGF signaling is necessary for establishing gut tube domains along the anterior-posterior axis in vivo. Mech Dev 123: 42-55.

Deutsch G, Jung J, Zheng M, Lora J, Zaret KS. (2001). A bipotential precursor population for pancreas and liver within the embryonic endoderm. Development 128: 871-881.

Dhawan S, Georgia S, Tschen SI, Fan G, Bhushan A. (2011). Pancreatic beta-cell identity is maintained by DNA methylation-mediated repression of Arx. Dev Cell 20: 419-429.

Dor Y, Brown J, Martinez OI, Melton DA. (2004). Adult pancreatic beta-cells are formed by selfduplication rather than stem-cell differentiation. Nature 429: 41-46.

Doyle MJ, Loomis ZL, Sussel L. (2007). Nkx2.2-repressor activity is sufficient to specify alphacells and a small number of beta-cells in the pancreatic islet. Development 134: 515-523.

Doyle MJ, Sussel L. (2007). Nkx2.2 regulates beta-cell function in the mature islet. Diabetes 56: 1999-2007.

Du A, Hunter CS, Murray J, Noble D, Cai CL, Evans SM, Stein R, May CL. (2009). Islet1 is required for the maturation, proliferation, and survival of the endocrine pancreas. Diabetes 58: 2059-2069. 
Duan D, Fu Y, Paxinos G, Watson C. (2013). Spatiotemporal expression patterns of Pax6 in the brain of embryonic, newborn, and adult mice. Brain Struct Funct 218: 353-372.

Dutta S, Gannon M, Peers B, Wright C, Bonner-Weir S, Montminy M. (2001). PDX:PBX complexes are required for normal proliferation of pancreatic cells during development. Proc Natl Acad Sci USA 98: 1065-1070.

Duvillie B, Attali M, Bounacer A, Ravassard P, Basmaciogullari A, Scharfmann R. (2006). The mesenchyme controls the timing of pancreatic beta-cell differentiation. Diabetes 55: 582-589.

Duvillié B, Cordonnier N, Deltour L, Dandoy-Dron F, Itier JM, Monthioux E, Jami J, Joshi RL, Bucchini D. (1997). Phenotypic alterations in insulin-deficient mutant mice. Proc Natl Acad Sci USA 94: 5137-5140.

Elghazi L, Cras-Meneur C, Czernichow P, Scharfmann R. (2002). Role for FGFR2IIIb-mediated signals in controlling pancreatic endocrine progenitor cell proliferation. Proc Natl Acad Sci USA 99: 3884-3889.

Farber CR, Chitwood J, Lee SN, Verdugo RA, Islas-Trejo A, Rincon G, Lindberg I, Medrano JF. (2008). Overexpression of Scg5 increases enzymatic activity of PCSK2 and is inversely correlated with body weight in congenic mice. BMC Genet 9: 34.

Fortenberry Y, Hwang JR, Apletalina EV, Lindberg I. (2002). Functional characterization of ProSAAS: similarities and differences with 7B2. J Biol Chem 277: 5175-5186.

Fortenberry Y, Liu J, Lindberg I. (1999). The role of the 7B2 CT peptide in the inhibition of prohormone convertase 2 in endocrine cell lines. J Neurochem 73: 994-1003.

Furuta M, Yano H, Zhou A, Rouillé Y, Holst JJ, Carroll R, Ravazzola M, Orci L, Furuta H, Steiner DF. (1997). Defective prohormone processing and altered pancreatic islet morphology in mice lacking active SPC2. Proc Natl Acad Sci USA 94: 6646-6651.

Gannon M, Herrera PL, Wright CV. (2000). Mosaic Cre-mediated recombination in pancreas using the Pdx-1 enhancer/promoter. Genesis 26: 143-144.

Gasa R, Mrejen C, Lynn FC, Skewes-Cox P, Sanchez L, Yang KY, Lin CH, Gomis R, German MS. (2008). Induction of pancreatic islet cell differentiation by the neurogenin-neuroD cascade. Differentiation 76: 381-391.

Gauthier BR, Gosmain Y, Mamin A, Philippe J. (2007). The $\beta$-cell specific transcription factor Nkx6.1 inhibits glucagon gene transcription by interfering with Pax6. Biochem J 403: 593-601.

Gelling RW, Du XQ, Dichmann DS, Romer J, Huang H, Cui L, Obici S, Tang B, Holst JJ, Fledelius C, Johansen PB, Rossetti L, Jelicks LA, Serup P, Nishimura E, Charron MJ. (2003). Lower blood glucose, hyperglucagonemia, and pancreatic alpha cell hyperplasia in glucagon receptor knockout mice. Proc Natl Acad Sci USA 100: 1438-1443.

Georgala PA, Carr CB, Price DJ. (2011). The role of Pax6 in forebrain development. Dev Neurobiol 71: 690-709.

Gittes GK, Galante PE, Hanahan D, Rutter WJ, Debase HT. (1996). Lineage-specific morphogenesis in the developing pancreas: role of mesenchymal factors. Development 122: 439-447.

Gittes GK. (2009). Developmental biology of the pancreas: a comprehensive review. Dev Biol 326: 4-35. 
Golosow N, Grobstein C. (1962). Epitheliomesenchymal interaction in pancreatic morphogenesis. Dev Biol 4: 242-255.

Goodge KA, Hutton JC. (2000). Translational regulation of proinsulin biosynthesis and proinsulin conversion in the pancreatic beta-cell. Semin Cell Dev Biol 11: 235-242.

Gosmain Y, Katz LS, Masson MH, Cheyssac C, Poisson C, Philippe J. (2012a). Pax6 is crucial for beta-cell function, insulin biosynthesis, and glucose-induced insulin secretion. Mol Endocrinol 26: 696-709.

Gosmain Y, Marthinet E, Cheyssac C, Guerardel A, Mamin A, Katz LS, Bouzakri K, Philippe J. (2010). Pax6 controls the expression of critical genes involved in pancreatic \{alpha\} cell differentiation and function. J Biol Chem 285: 33381-33393.

Gosmain Y, Cheyssac C, Masson MH, Guérardel A, Poisson C, Philippe J. (2012b). Pax6 is a key component of regulated glucagon secretion. Endocrinology 153: 4204-4215.

Gradwohl G, Dierich A, LeMeur M, Guillemot F. (2000). Neurogenin3 is required for the development of the four endocrine cell lineages of the pancreas. Proc Natl Acad Sci USA 97: 1607-1611.

Granata R, Settanni F, Biancone L, Trovato L, Nano R, Bertuzzi F, Destefanis S, Annunziata M, Martinetti M, Catapano F, Ghè C, Isgaard J, Papotti M, Ghigo E, Muccioli G. (2007). Acylated and unacylated ghrelin promote proliferation and inhibit apoptosis of pancreatic beta-cells and human islets: involvement of 3',5'-cyclic adenosine monophosphate/protein kinase A, extracellular signal-regulated kinase 1/2, and phosphatidyl inositol 3-Kinase/Akt signaling. Endocrinology 148: 512-529.

Granata R, Settanni F, Trovato L, Destefanis S, Gallo D, Martinetti M, Ghigo E, Muccioli G. (2006). Unacylated as well as acylated ghrelin promotes cell survival and inhibit apoptosis in HIT-T15 pancreatic beta-cells. J Endocrinol Invest 29: 19-22.

Grapin-Botton A, Melton DA. (2000). Endoderm development: from patterning to organogenesis. Trends Genet 16: 124-130.

Gu C, Stein GH, Pan N, Goebbels S, Hornberg H, Nave KA, Herrera P, White P, Kaestner KH, Sussel L, Lee JE. (2010). Pancreatic beta-cells require NeuroD to achieve and maintain functional maturity. Cell Metab 11: 298-310.

Gu G, Dubauskaite J, Melton DA. (2002). Direct evidence for the pancreatic lineage: NGN3+ cells are islet progenitors and are distinct from duct progenitors. Development 129: 2447-2457.

Gualdi R, Bossard P, Zheng M, Hamada Y, Coleman JR, Zaret KS. (1996). Hepatic specification of the gut endoderm in vitro: cell signaling and transcriptional control. Genes Dev 10: 16701682.

Guillam MT, Hümmler E, Schaerer E, Yeh JI, Birnbaum MJ, Beermann F, Schmidt A, Dériaz N, Thorens B. (1997). Early diabetes and abnormal postnatal pancreatic islet development in mice lacking Glut-2. Nat Genet 17: 327-330.

Guz Y, Montminy MR, Stein R, Leonard J, Gamer LW, Wright CV, Teitelman G. (1995). Expression of murine STF-1, a putative insulin gene transcription factor, in beta-cells of pancreas, duodenal epithelium and pancreatic exocrine and endocrine progenitors during ontogeny. Development 121: 11-18. 
Hale MA, Kagami H, Shi L, Holland AM, Elsasser HP, Hammer RE, MacDonald RJ. (2005). The homeodomain protein PDX1 is required at mid-pancreatic development for the formation of the exocrine pancreas. Dev Biol 286: 225-237.

Harrison KA, Thaler J, Pfaff SL, Gu H, Kehrl JH. (1999). Pancreas dorsal lobe agenesis and abnormal islets of Langerhans in Hlxb9-deficient mice. Nat Genet 23: 71-75.

Hart AW, Mella S, Mendrychowski J, van Heyningen V, Kleinjan DA. (2013). The developmental regulator Pax6 is essential for maintenance of islet cell function in the adult mouse pancreas. PLoS One 8: e54173.

Hauge-Evans AC, King AJ, Carmignac D, Richardson CC, Robinson IC, Low MJ, Christie MR, Persaud SJ, Jones PM. (2009). Somatostatin secreted by islet delta-cells fulfills multiple roles as a paracrine regulator of islet function. Diabetes 58: 403-411.

Hebrok M, Kim SK, Melton DA. (1998). Notochord repression of endodermal Sonic hedgehog permits pancreas development. Genes Dev 12: 1705-1713.

Heller RS, Jenny M, Collombat P, Mansouri A, Tomasetto C, Madsen OD, Mellitzer G, Gradwohl G, Serup P. (2005). Genetic determinants of pancreatic epsilon-cell development. Dev Biol 286: 217-224.

Helwig M, Hoshino A, Berridge C, Lee SN, Lorenzen N, Otzen DE, Eriksen JL, Lindberg I. (2013). The neuroendocrine protein 7B2 suppresses the aggregation of neurodegenerative disease-related proteins. J Biol Chem 288: 1114-1124.

Helwig M, Lee SN, Hwang JR, Ozawa A, Medrano JF, Lindberg I. (2011). Dynamic modulation of prohormone convertase 2 (PC2)-mediated precursor processing by 7B2 protein: preferential effect on glucagon synthesis. J Biol Chem 286: 42504-42513.

Herrera PL. (2000). Adult insulin- and glucagon-producing cells differentiate from two independent cell lineages. Development 127: 2317-2322.

Hill JT, Mastracci TL, Vinton C, Doyle ML, Anderson KR, Loomis ZL, Schrunk JM, Minic AD, Prabakar KR, Pugliese A, Sun Y, Smith RG, Sussel L. (2009). Ghrelin is dispensable for embryonic pancreatic islet development and differentiation. Regul Pept 157: 51-56.

Hill RE, Favor J, Hogan BL, Ton CC, Saunders GF, Hanson IM, Prosser J, Jordan T, Hastie ND, van Heyningen V. (1991). Mouse small eye results from mutations in a paired-like homeobox-containing gene. Nature 354: 522-525.

Hodgson SV, Saunders KE. (1980). A probable case of the homozygous condition of the aniridia gene. J Med Genet 6: 476-480.

Holland AM, Hale MA, Kagami H, Hammer RE, MacDonald RJ. (2002). Experimental control of pancreatic development and maintenance. Proc Natl Acad Sci USA 99: 12236-12241.

Huang HP, Liu M, El-Hodiri HM, Chu K, Jamrich M, Tsai MJ. (2000). Regulation of the pancreatic islet-specific gene BETA2 (neuroD) by neurogenin 3. Mol Cell Biol 20: 3292-3307.

Hull RL, Westermark GT, Westermark P, Kahn SE. (2004). Islet amyloid: a critical entity in the pathogenesis of type 2 diabetes. J Clin Endocrinol Metab 89: 3629-3643.

Inui A, Asakawa A, Bowers CY, Mantovani G, Laviano A, Meguid MM, Fujimiya M. (2004). Ghrelin, appetite, and gastric motility: the emerging role of the stomach as an endocrine organ. FASEB J 18: 439-456. 
Itkin-Ansari P, Marcora E, Geron I, Tyrberg B, Demeterco C, Hao E, Padilla C, Ratineau C, Leiter A, Lee JE, Levine F. (2005). NeuroD1 in the endocrine pancreas: localization and dual function as an activator and repressor. Dev Dyn 233: 946-953.

Jensen J, Heller RS, Funder-Nielsen T, Pedersen EE, Lindsell C, Weinmaster G, Madsen OD, Serup P. (2000a). Independent development of pancreatic alpha- and beta-cells from neurogenin3-expressing precursors: a role for the notch pathway in repression of premature differentiation. Diabetes 49: 163-176.

Jensen J, Pedersen EE, Galante P, Hald J, Heller RS, Ishibashi M, Kageyama R, Guillemot F, Serup P, Madsen OD. (2000b). Control of endodermal endocrine development by Hes-1. Nat Genet 24: 36-44.

Jensen MV, Joseph JW, Ronnebaum SM, Burgess SC, Sherry AD, Newgard CB. (2008). Metabolic cycling in control of glucose-stimulated insulin secretion. Am J Physiol Endocrinol Metab 295: E1287-97.

Jeon J, Correa-Medina M, Ricordi C, Edlund H, Diez JA. (2009). Endocrine cell clustering during human pancreas development. J Histochem Cytochem 57: 811-824.

Johansson KA, Dursun U, Jordan $\quad$ N, Gu G, Beermann ～F, Gradwohl G, Grapin-Botton A. (2007). Temporal control of neurogenin3 activity in pancreas progenitors reveals competence windows for the generation of different endocrine cell types. Dev Cell 12: 457-465.

Jonsson J, Ahlgren U, Edlund T, Edlund H. (1995). IPF1, a homeodomain protein with a dual function in pancreas development. Int J Dev Biol 39: 789-798.

Jonsson J, Carlsson L, Edlund T, Edlund H. (1994). Insulin-promoter-factor 1 is required for pancreas development in mice. Nature 371: 606-609.

Jørgensen MC, Ahnfelt-Rønne J, Hald J, Madsen OD, Serup P, Hecksher-Sørensen J. (2007). An illustrated review of early pancreas development in the mouse. Endocr Rev 28: 685-705.

Jung J, Zheng M, Goldfarb M, Zaret KS. (1999). Initiation of mammalian liver development from endoderm by fibroblast growth factors. Science 284: 1998-2003.

Kahn SE, Andrikopoulos S, Verchere CB. (1999). Islet amyloid: a long-recognized but underappreciated pathological feature of type 2 diabetes. Diabetes 48: 241-253.

Katz LS, Gosmain Y, Marthinet E, Philippe J. (2009). Pax6 regulates the proglucagon processing enzyme PC2 and its chaperone 7B2. Mol Cell Biol 29: 2322-2334.

Kawaguchi Y, Cooper B, Gannon M, Ray M, MacDonald RJ, Wright CV. (2002). The role of the transcriptional regulator Ptf1a in converting intestinal to pancreatic progenitors. Nat Genet 32: 128-134.

Kim SK, Selleri L, Lee JS, Zhang AY, Gu X, Jacobs Y, Cleary ML. (2002). Pbx1 inactivation disrupts pancreas development and in Ipf1-deficient mice promotes diabetes mellitus. Nat Genet 30: 430-435.

Kitamura T, Kahn CR, Accili D. (2003). Insulin receptor knockout mice. Annu Rev Physiol 65: 313-332.

Kojima M, Hosoda H, Date Y, Nakazato M, Matsuo H, Kangawa K. (1999). Ghrelin is a growthhormone releasing acylated peptide from stomach. Nature 402: 656-660. 
Kojima S, Ueno N, Asakawa A, Sagiyama K, Naruo T, Mizuno S, Inui A. (2007). A role for pancreatic polypeptide in feeding and body weight regulation. Peptides 28: 459-463.

Kordowich S, Collombat P, Mansouri A, Serup P. (2011). Arx and Nkx2.2 compound deficiency redirects pancreatic alpha- and beta-cell differentiation to a somatostatin/ghrelin co-expressing cell lineage. BMC Dev Biol 11: 52.

Kramer J, Moeller EL, Hachey A, Mansfield KG, Wachtman LM. (2009). Differential expression of GLUT2 in pancreatic islets and kidneys of New and Old World nonhuman primates. Am J Physiol Regul Integr Comp Physiol 296: R786-93.

Krapp A, Knofler M, Frutiger S, Hughes GJ, Hagenbuchle O, Wellauer PK. (1996). The p48 DNA-binding subunit of transcription factor PTF1 is a new exocrine pancreas-specific basic helix-loop-helix protein. EMBO J 15: 4317-4329.

Krapp A, Knofler M, Ledermann B, Burki K, Berney C, Zoerkler N, Hagenbuchle O, Wellauer PK. (1998). The bHLH protein PTF1-p48 is essential for the formation of the exocrine and the correct spatial organization of the endocrine pancreas. Genes Dev 12: 3752-3763.

Kredo-Russo S, Mandelbaum AD, Ness A, Alon I, Lennox KA, Behlke MA, Hornstein E. (2012). Pancreas-enriched miRNA refines endocrine cell differentiation. Development 139: 3021-3031.

Larsson LI, Madsen OD, Serup P, Jonsson J, Edlund H. (1996). Pancreatic-duodenal homeobox 1 role in gastric endocrine patterning. Mech Dev 60: 175-184.

Leighton B, Cooper GJ. (1988). Pancreatic amylin and calcitonin gene-related peptide cause resistance to insulin in skeletal muscle in vitro. Nature 335: 632-635.

Li H, Arber S, Jessell TM, Edlund H. (1999). Selective agenesis of the dorsal pancreas in mice lacking homeobox gene Hlxb9. Nat Genet 23: 67-70.

Li H, Edlund H. (2001). Persistent expression of Hlxb9 in the pancreatic epithelium impairs pancreatic development. Dev Biol 240: 247-253.

Liu T, Zhao Y, Tang N, Feng R, Yang X, Lu N, Wen J, Li L. (2012). Pax6 directly downregulates Pcsk1n expression thereby regulating PC1/3 dependent proinsulin processing. PLoS One 7: e46934.

Liu Y, MacDonald RJ, Swift GH. (2001). DNA binding and transcriptional activation by a PDX1.PBX1b.MEIS2b trimer and cooperation with a pancreas-specific basic helix-loop-helix complex. J Bio Chem 276: 17985-17993.

Lynn FC, Smith SB, Wilson ME, Yang KY, Nekrep N, German MS. (2007). Sox9 coordinates a transcriptional network in pancreatic progenitor cells. Proc Natl Acad Sci USA 104: 1050010505.

MacDonald PE, El-Kholy W, Riedel MJ, Salapatek AM, Light PE, Wheeler MB. (2002). The multiple actions of GLP-1 on the process of glucose-stimulated insulin secretion. Diabetes 51: S434-42.

MacFarlane WM, Read ML, Gilligan M, Bujalska I, Docherty K. (1994). Glucose modulates the binding activity of the beta-cell transcription factor IUF1 in a phosphorylation-dependent manner. Biochem J 303: 625-631. 
Malecki MT, Jhala US, Antonellis A, Fields L, Doria A, Orban T, Saad M, Warram JH, Montminy M, Krolewski AS. (1999). Mutations in NEUROD1 are associated with the development of type 2 diabetes mellitus. Nat Genet 23: 323-328.

Manuel M, Pratt T, Liu M, Jeffery G, Price DJ. (2008). Overexpression of Pax6 results in microphthalmia, retinal dysplasia and defective retinal ganglion cell exon guidance. BMC Dev Biol 8: 59.

Marcinkiewicz M, Ramla D, Seidah NG, Chrétien M. (1994). Developmental expression of the prohomone convertases PC1 and PC2 in mouse pancreatic islets. Endocrinology 135: 16511660.

Marshak S, Totary H, Cerasi E, Melloul D. (1996). Purification of the beta-cell glucose-sensitive factor that transactivates the insulin gene differentially in normal and transformed islet cells. Proc Natl Acad Sci USA 93: 15057-15062.

Marzban L, Trigo-Gonzalez G, Zhu X, Rhodes CJ, Halban PA, Steiner DF, Verchere CB. (2004). Role of beta-cell prohormone convertase (PC)1/3 in processing of pro-islet amyloid polypeptide. Diabetes 53: 141-148.

Mastracci TL, Lin CS, Sussel L. (2013). Generation of mice encoding a conditional allele of Nkx2.2. Transgenic Res [Epub ahead of print].

Mastracci TL, Sussel L. (2012). The Endocrine Pancreas: insights into development, differentiation and diabetes. Wiley Interdiscip Rev Membr Transp Signal 1: 609-628.

Mastracci TL, Wilcox CL, Arnes L, Panea C, Golden JA, May CL, Sussel L. (2011). Nkx2.2 and Arx genetically interact to regulate pancreatic endocrine cell development and endocrine hormone expression. Dev Biol 359: 1-11.

Matsuoka TA, Kaneto H, Stein R, Miyatsuka T, Kawamori D, Henderson E, Kojima I, Matsuhisa M, Hori M, Yamasaki Y (2007). MafA regulates expression of genes important to islet beta-cell function. Mol Endocrinol 21: 2764-2774.

Mellitzer G, Bonne S, Luco RF, Van De Casteele M, Lenne-Samuel N, Collombat P, Mansouri A, Lee J, Lan M, Pipeleers D, Nielsen FC, Ferrer J, Gradwohl G, Heimberg H. (2006). IA1 is NGN3-dependent and essential for differentiation of the endocrine pancreas. EMBO J 25: 13441352.

Miralles F, Czernichow P, Ozaki K, Itoh N, Scharfmann R. (1999). Signaling through fibroblast growth factor receptor $2 \mathrm{~b}$ plays a key role in the development of the exocrine pancreas. Proc Natl Acad Sci USA 96: 6267-6272.

Miyatsuka T, Kaneto H, Shiraiwa T, Matsuoka TA, Yamamoto K, Kato K, Nakamura Y, Akira S, Takeda K, Kajimoto Y, Yamasaki Y, Sandgren EP, Kawaguchi Y, Wright CV, Fujitani Y. (2006). Persistent expression of PDX-1 in the pancreas causes acinar-to-ductal metaplasia through Stat3 activation. Genes Dev 20: 1435-1440.

Molven A, Ringdal M, Nordbø AM, Raeder H, Støy J, Lipkind GM, Steiner DF, Philipson LH, Bergmann I, Aarskog D, Undlien DE, Joner G, Søvik O; Norwegian Childhood Diabetes Study Group, Bell GI, Njølstad PR. (2008). Mutations in the insulin gene can cause MODY and autoantibody-negative type 1 diabetes. Diabetes 57: 1131-1135.

Najjar, S. (2003). Insulin Action: Molecular Basis of Diabetes. eLS. 
Naya FJ, Huang HP, Qiu Y, Mutoh H, DeMayo FJ, Leiter AB, Tsai MJ. (1997). Diabetes, defective pancreatic morphogenesis, and abnormal enteroendocrine differentiation in BETA2/neuroD-deficient mice. Genes Dev 11: 2323-2334.

Nir T, Melton DA, Dor Y. (2007). Recovery from diabetes in mice by beta-cell regeneration. J Clin Invset 117: 2553-2561.

Nishimura W, Kondo T, Salameh T, El Khattabi I, Dodge R, Bonner-Weir S, Sharma A. (2006). A switch from MafB to MafA expression accompanies differentiation to pancreatic beta-cells. Dev Biol 293: 526-539.

Offield MF, Jetton TL, Labosky PA, Ray M, Stein RW, Magnuson MA, Hogan BL, Wright CV. (1996). PDX-1 is required for pancreatic outgrowth and differentiation of the rostral duodenum. Development 122: 983-995.

Oron-Karni V, Farhy C, Elgart M, Marquardt T, Remizova L, Yaron O, Xie Q, Cvekl A, AsheryPadan R. (2008). Dual requirement for Pax6 in retinal progenitor cells. Development 135: 40374047.

Osumi N, Shinohara H, Numayama-Tsuruta K, Maekawa M. (2008). Concise review: Pax6 transcription factor contributes to both embryonic and adult neurogenesis as a multifunctional regulator. Stem Cells 26: 1663-1672.

Pan FC, Wright C. (2011). Pancreas organogenesis: From bud to plexus to gland. Dev Dyn 240: 530-565.

Papizan JB, Singer RA, Tschen SI, Dhawan S, Friel JM, Hipkens SB, Magnuson MA, Bhushan A, Sussel L. (2011). Nkx2.2 repressor complex regulates islet $\beta$-cell specification and prevents $\beta$-to- $\alpha$-cell reprogramming. Genes Dev 25: 2291-2305.

Piñon MC, Tuoc TC, Ashery-Padan R, Molnár Z, Stoykova A. (2008). Altered molecular regionalization and normal thalamocortical connections in cortex-specific Pax6 knock-out mice. J Neurosci 28: 8724-8734.

Prado CL, Pugh-Bernard AE, Elghazi L, Sosa-Pineda B, Sussel L. (2004). Ghrelin cells replace insulin-producing beta-cells in two mouse models of pancreas development. Proc Natl Acad Sci USA 101: 2924-2929.

Prelich G. (2012). Gene overexpression: uses, mechanisms, and interpretation. Genetics 190: 841854.

Prosser J, van Heyningen V. (1998). PAX6 mutations reviewed. Hum Mutat 11: 93-108.

Quinn JC, Molinek M, Martynoga BS, Zaki PA, Faedo A, Bulfone A, Hevner RF, West JD, Price DJ. (2007). Pax6 controls cerebral cortical cell number by regulating exit from the cell cycle and specifies cortical cell identity by a cell autonomous mechanism. Dev Biol 302: 50-65.

Rajewsky K, Gu H, Kühn R, Betz UA, Müller W, Roes J, Schwenk F. (1996). Conditional gene targeting. J Clin Invest 98: 600-603.

Raum JC, Gerrish K, Artner I, Henderson E, Guo M, Sussel L, Schisler JC, Newgard CB, Stein R. (2006). FoxA2, Nkx2.2, and PDX-1 regulate islet beta-cell-specific mafA expression through conserved sequences located between base pairs -8118 and -7750 upstream from the transcription start site. Mol Cell Biol 26: 5735-5743. 
Raum JC, Hunter CS, Artner I, Henderson E, Guo M, Elghazi L, Sosa-Pineda B, Ogihara T, Mirmira RG, Sussel L, Stein R. (2010). Islet beta-cell-specific MafA transcription requires the 5'-flanking conserved region 3 control domain. Mol Cell Biol 30: 4234-4244.

Reza HM, Ogino H, Yasuda K. (2002). L-Maf, a downstream target of Pax6, is essential for chick lens development. Mech Dev 116: 61-73.

Rose SD, Kruse F, Swift GH, MacDonald RJ, Hammer RE. (1994). A single element of the elastase I enhancer is sufficient to direct transcription selectively to the pancreas and gut. Mol Cell Biol 14: 2048-2057.

Rossi JM, Dunn NR, Hogan BL, Zaret KS. (2001). Distinct mesodermal signals, including BMPs from the septum transversum mesenchyme, are required in combination for hepatogenesis from the endoderm. Genes Dev 15: 1998-2009.

Rubio-Cabezas O, Minton JA, Kantor I, Williams D, Ellard S, Hattersley AT. (2010). Homozygous mutations in NEUROD1 are responsible for a novel syndrome of permanent neonatal diabetes and neurological abnormalities. Diabetes 59: 2326-2331.

Sakurai K., Osumi N. (2008). The neurogenesis-controlling factor, Pax6, inhibits proliferation and promotes maturation in murine astrocytes. J Neurosci 28: 4604-4612.

Sander M, Neubüser A, Kalamaras J, Ee HC, Martin GR, German MS. (1997). Genetic analysis reveals that PAX6 is required for normal transcription of pancreatic hormone genes and islet development. Genes Dev 11: 1662-1673.

Sander M, Sussel L, Conners J, Scheel D, Kalamaras J, Dela Cruz F, Schwitzgebel V, HayesJordan A, German M. (2000). Homeobox gene Nkx6.1 lies downstream of Nkx2.2 in the major pathway of beta-cell formation in the pancreas. Development 127: 5533-5540.

Sauer B, Henderson N. (1989). Cre-stimulated recombination at loxP-containing DNA sequences placed into the mammalian genome. Nucleic Acids Res 17: 147-161.

Schaffer AE, Taylor BL, Benthuysen JR, Liu J, Thorel F, Yuan W, Jiao Y, Kaestner KH, Herrera PL, Magnuson MA, May CL, Sander M. (2013). Nkx6.1 controls a gene regulatory network required for establishing and maintaining pancreatic beta-cell identity. PLoS Genet 9: e1003274.

Schedl A, Ross A, Lee M, Engelkamp D, Rashbass P, van Heyningen V, Hastie ND. (1996). Influence of PAX6 gene dosage on development: overexpression causes severe eye abnormalities. Cell 86: 71-82.

Schwitzgebel VM, Scheel DW, Conners JR, Kalamaras J, Lee JE, Anderson DJ, Sussel L, Johnson JD, German MS. (2000).Expression of neurogenin3 reveals an islet cell precursor population in the pancreas. Development 127: 3533-3542.

Sellick GS, Barker KT, Stolte-Dijkstra I, Fleischmann C, Coleman RJ, Garrett C, Gloyn AL, Edghill EL, Hattersley AT, Wellauer PK,Goodwin G, Houlston RS. (2004). Mutations in PTF1A cause pancreatic and cerebellar agenesis. Nat Genet 36: 1301-1305.

Seymour PA, Freude $\quad$ KK., Tran $\quad$ MN, Mayes EE, Jensen J, Kist R, Scherer $\quad$ G, Sander M. (2007). SOX9 is required for maintenance of the pancreatic progenitor cell pool. Proc Natl Acad Sci USA 104: 1865-1870.

Shaham, O., Menuchin, Y., Farhy, C., Ashery-Padan, R. (2012). Pax6: A multi-level regulator of ocular development. Prog Retin Eye Res 31: 351-376. 
Sherwood RI, Chen TY, Melton DA. (2009). Transcriptional dynamics of endodermal organ formation. Dev Dyn 238: 29-42.

Slingerland AS. (2006). Monogenic diabetes in children and young adults:Challenges for researcher, clinician and patient. Rev Endocr Metab Disord 7: 171-185.

Smith SB, Ee HC, Conners JR, German MS. (1999). Paired-homeodomain transcription factor PAX4 acts as a transcriptional repressor in early pancreatic development. Mol Cell Biol 19: 8272-8280.

Smith SB, Gasa R, Watada H, Wang J, Griffen SC, German MS. (2003). Neurogenin3 and hepatic nuclear factor 1 cooperate in activating pancreatic expression of Pax4. J Biol Chem 278: 3825438259 .

Smith SB, Qu HQ, Taleb N, Kishimoto NY, Scheel DW, Lu Y, Patch AM, Grabs R, Wang J, Lynn FC, Miyatsuka T, Mitchell J, Seerke R, Désir J, Vanden Eijnden S, Abramowicz M, Kacet N, Weill J, Renard ME, Gentile M, Hansen I, Dewar K, Hattersley AT, Wang R, Wilson ME, Johnson JD, Polychronakos C, German MS. (2010). Rfx6 directs islet formation and insulin production in mice and humans. Nature 463: 775-780.

Smith SB, Watada H, German MS. (2004). Neurogenin3 activates the islet differentiation program while repressing its own expression. Mol Endocrinol 18: 142-149.

Sosa-Pineda B, Chowdhury K, Torres M, Oliver G, Gruss P. (1997). The Pax4 gene is essential for differentiation of insulin-producing beta-cells in the mammalian pancreas. Nature 386: 399402.

Sowa R, Sanke T, Hirayama J, Tabata H, Furuta H, Nishimura S, Nanjo K. (1990). Islet amyloid polypeptide amide causes peripheral insulin resistance in vivo in dogs. Diabetologia 33: 118120.

Soyer J, Flasse L, Raffelsberger W, Beucher A, Orvain C, Peers B, Ravassard P, Vermot J, Voz ML, Mellitzer G, Gradwohl G. (2010). Rfx6 is an Ngn3-dependent winged helix transcription factor required for pancreatic islet cell development. Development 137: 203-212.

Srinivas S, Watanabe T, Lin CS, William CM, Tanabe Y, Jessell TM, Costantini F. (2001). Cre reporter strains produced by targeted insertion of EYFP and ECFP into the ROSA26 locus. BMC Dev Biol 1: 4.

Stafford D, Hornbruch A, Mueller PR, Prince VE. (2004). A conserved role for retinoid signaling in vertebrate pancreas development. Dev Genes Evol 214: 432-441.

Stafford D, White RJ, Kinkel MD, Linville A, Schilling TF, Prince VE. (2006). Retinoids signal directly to zebrafish endoderm to specify insulin-expressing beta-cells. Development 133: 949956.

Stanger BZ, Tanaka AJ, Melton DA. (2007). Organ size is limited by the number of embryonic progenitor cells in the pancreas but not the liver. Nature 445: 886-891.

Stoffers DA, Ferrer J, Clarke WL, Habener JF. (1997b). Early-onset type-II diabetes mellitus (MODY4) linked to IPF1. Nat Genet 17: 138-139.

Stoffers DA, Zinkin NT, Stanojevic V, Clarke WL, Habener JF. (1997a). Pancreatic agenesis attributable to a single nucleotide deletion in the human IPF1 gene coding sequence. Nat Genet 15: 106-110. 
St-Onge L, Sosa-Pineda B, Chowdhury K, Mansouri A, Gruss P. (1997). Pax6 is required for differentiation of glucagon-producing alpha-cells in mouse pancreas. Nature 387: 406-409.

Stumvoll M, Goldstein BJ, van Haeften TW. (2005). Type 2 diabetes: principles of pathogenesis and therapy. Lancet 365: 1333-1346.

Sussel L, Kalamaras J, Hartigan-O'Connor DJ, Meneses JJ, Pedersen RA, Rubenstein JL, German MS. (1998). Mice lacking the homeodomain transcription factor Nkx2.2 have diabetes due to arrested differentiation of pancreatic beta-cells. Development 125: 2213-2221.

Swift GH, Liu Y, Rose SD, Bischof LJ, Steelman S, Buchberg AM, Wright CV, MacDonald RJ. (1998). An endocrine-exocrine switch in the activity of the pancreatic homeodomain protein PDX1 through formation of a trimeric complex with PBX1b and MRG1 (MEIS2). Mol Cell Biol 18: 5109-5120.

Thaler J, Harrison K, Sharma K, Lettieri K, Kehrl J, Pfaff SL. (1999). Active suppression of interneuron programs within developing motor neurons revealed by analysis of homeodomain factor HB9. Neuron 23: 675-687.

Thorel F, Népote V, Avril I, Kohno K, Desgraz R, Chera S, Herrera PL. (2010). Conversion of adult pancreatic alpha-cells to beta-cells after extreme beta-cell loss. Nature 464: 1149-1154.

Thorens B, Wu YJ, Leahy JL, Weir GC. (1992). The loss of GLUT2 expression by glucoseunresponsive beta-cells of $\mathrm{db} / \mathrm{db}$ mice is reversible and is induced by the diabetic environment. J Clin Invest 90: 77-85.

Ton CC, Hirvonen H, Miwa H, Weil MM, Monaghan P, Jordan T, van Heyningen V, Hastie ND, Meijers-Heijboer H, Drechsler M, Royer-Pokora B, Collins F, Swaroop A, Strong LC, Saunders GF. (1991). Positional cloning and characterization of a paired box- and homeobox-containing gene from the aniridia region. Cell 67: 1059-1074.

Vasic D, Walcher D. (2012). C-peptide: a new mediator of atherosclerosis in diabetes. Mediators Inflamm 2012: 858692.

Villasenor A, Chong DC, Cleaver O. (2008). Biphasic Ngn3 expression in the developing pancreas. Dev Dyn 237: 3270-3279.

Waha A, Koch A, Hartmann W, Milde U, Felsberg J, Hübner A, Mikeska T, Goodyer CG, Sörensen N, Lindberg I, Wiestler OD, Pietsch T, Waha A. (2007). SGNE1/7B2 is epigenetically altered and transcriptionally downregulated in human medulloblastomas. Oncogene 26: 5662-5668.

Walther C, Gruss P. (1991). Pax-6, a murine paired box gene, is expressed in the developing CNS. Development 113: 1435-1449.

Wang Q, Fang WH, Krupinski J, Kumar S, Slevin M, Kumar P. (2008). Pax genes in embryogenesis and oncogenesis. J Cell Mol Med 12: 2281-2294.

Wang S, Yan J, Anderson DA, Xu Y, Kanal MC, Cao Z, Wright CV, Gu G. (2010). Neurog3 gene dosage regulates allocation of endocrine and exocrine cell fates in the developing mouse pancreas. Dev Biol 339: 26-37.

Wei S, Feng Y, Che FY, Pan H, Mzhavia N, Devi LA, McKinzie AA, Levin N, Richards WG, Fricker LD. (2004). Obesity and diabetes in transgenic mice expressing proSAAS. J Endocrinol 180: 357-368. 
Wells JM, Melton DA. (2000). Early mouse endoderm is patterned by soluble factors from adjacent germ layers. Development 127: 1563-1572.

Wen JH, Chen YY, Song SJ, Ding J, Gao Y, Hu QK, Feng RP, Liu YZ, Ren GC, Zhang CY, Hong TP, Gao X, Li LS. (2009). Paired box 6 (PAX6) regulates glucose metabolism via proinsulin processing mediated by prohormone convertase 1/3 (PC1/3). Diabetologia 52: 504513.

Wen JH, Chen YY, Song SJ, Ding J, Gao Y, Hu QK, Feng RP, Liu YZ, Ren GC, Zhang CY, Hong TP, Gao X, Li LS. (2009). Paired box 6 (PAX6) regulates glucose metabolism via proinsulin processing mediated by prohormone convertase 1/3 (PC1/3). Diabetologia 52: 504513.

Westphal CH, Muller L, Zhou A, Zhu X, Bonner-Weir S, Schambelan M, Steiner DF, Lindberg I, Leder P. (1999). The neuroendocrine protein 7B2 is required for peptide hormone processing in vivo and provides a novel mechanism for pituitary Cushing's disease. Cell 96: 689-700.

Wideman RD, Kieffer TJ. (2009). Mining incretin hormone pathways for novel therapies. Trends Endocrinol Metab 20: 280-286.

Wierup N, Yang S, McEvilly RJ, Mulder H, Sundler F. (2004). Ghrelin is expressed in a novel endocrine cell type in developing rat islets and inhibits insulin secretion from INS-1 (832/13) cells. J Histochem Cytochem 52: 301-310.

Wu KL, Gannon M, Peshavaria M, Offield MF, Henderson E, Ray M, Marks A, Gamer LW, Wright CV, Stein R. (1997). Hepatocyte nuclear factor 3beta is involved in pancreatic beta-cellspecific transcription of the pdx-1 gene. Mol Cell Biol 17: 6002-6013.

Xie Q, Yang Y, Huang J, Ninkovic J, Walcher T, Wolf L, Vitenzon A, Zheng D, Götz M, Beebe DC, Zavadil J, Cvekl A. (2013). Pax6 interactions with chromatin and identification of its novel direct target genes in lens and forebrain. PLoS One 8: e54507.

Yamaoka T, Yano M, Yamada T, Matsushita T, Moritani M, Ii S, Yoshimoto K, Hata J, Itakura M. (2000). Diabetes and pancreatic tumours in transgenic mice expressing Pax6. Diabetologia 43: 332-339.

Yang YP, Thorel F, Boyer DF, Herrera PL, Wright CV. (2011). Context-specific $\alpha$ - to- $\beta$-cell reprogramming by forced Pdx1 expression. Genes Dev 25: 1680-1685.

Yasuda T, Kajimoto Y, Fujitani Y, Watada H, Yamamoto S, Watarai T, Umayahara Y, Matsuhisa M, Gorogawa S, Kuwayama Y, Tano Y, Yamasaki Y, Hori M. (2002). PAX6 mutation as a genetic factor common to aniridia and glucose intolerance. Diabetes 51: 224-230.

Zhang C, Moriguchi T, Kajihara M, Esaki R, Harada A, Shimohata H, Oishi H, Hamada M, Morito N, Hasegawa K, Kudo T, Engel JD, Yamamoto M, Takahashi S. (2005). MafA is a key regulator of glucose-stimulated insulin secretion. Mol Cell Biol 25: 4969-4976.

Zhang Y, Zhang Y, Bone RN, Cui W, Peng JB, Siegal GP, Wang H, Wu H. (2012). Regeneration of pancreatic non-beta endocrine cells in adult mice following a single diabetes-inducing dose of streptozotocin. PLoS One 7: e36675.

Zhu X, Orci L, Carroll R, Norrbom C, Ravazzola M, Steiner DF. (2002). Severe block in processing of proinsulin to insulin accompanied by elevation of des-64,65 proinsulin intermediates in islets of mice lacking prohormone converatse 1/3. Proc Natl Acad Sci USA 99: 10299-10304. 


\section{Websites used:}

http://www.olivelab.org/the-pancreas-overview.html

http://www.betacell.org/content/articleview/article_id/13?aid=13

http://www.ncbi.nlm.nih.gov/pubmedhealth/PMH0002194/

http://www.genomatix.de/ 